\title{
Neurodevelopmental toxicity detection by transcriptomics in an embryonic stem cell differentiation assay
}

Citation for published version (APA):

Theunissen, P. T. (2013). Neurodevelopmental toxicity detection by transcriptomics in an embryonic stem cell differentiation assay. [Doctoral Thesis, Maastricht University]. Maastricht University. https://doi.org/10.26481/dis.20130301pt

Document status and date:

Published: 01/01/2013

DOI:

10.26481/dis.20130301pt

Document Version:

Publisher's PDF, also known as Version of record

Please check the document version of this publication:

- A submitted manuscript is the version of the article upon submission and before peer-review. There can be important differences between the submitted version and the official published version of record.

People interested in the research are advised to contact the author for the final version of the publication, or visit the DOI to the publisher's website.

- The final author version and the galley proof are versions of the publication after peer review.

- The final published version features the final layout of the paper including the volume, issue and page numbers.

Link to publication

\footnotetext{
General rights rights.

- You may freely distribute the URL identifying the publication in the public portal. please follow below link for the End User Agreement:

www.umlib.nl/taverne-license

Take down policy

If you believe that this document breaches copyright please contact us at:

repository@maastrichtuniversity.nl

providing details and we will investigate your claim.
}

Copyright and moral rights for the publications made accessible in the public portal are retained by the authors and/or other copyright owners and it is a condition of accessing publications that users recognise and abide by the legal requirements associated with these

- Users may download and print one copy of any publication from the public portal for the purpose of private study or research.

- You may not further distribute the material or use it for any profit-making activity or commercial gain

If the publication is distributed under the terms of Article $25 \mathrm{fa}$ of the Dutch Copyright Act, indicated by the "Taverne" license above, 


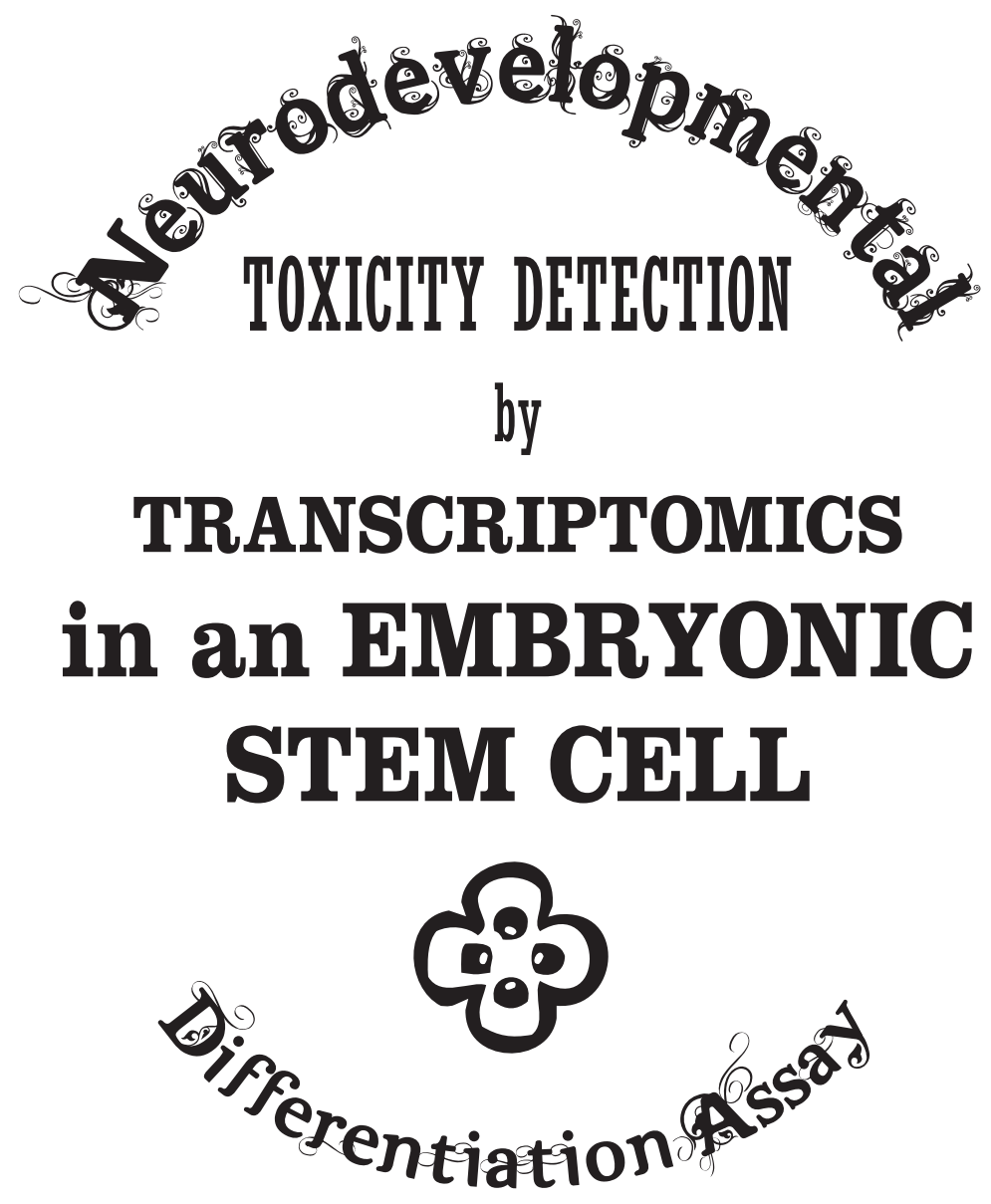

PETER T. THEUNISSEN 


\section{Neurodevelopmental toxicity detection by transcriptomics in an embryonic stem cell differentiation assay}

\section{Proefschrift}

This research was financially supported by the Dutch Technology Foundation STW (MFA6809)

Financial support of the printing costs of this thesis by the National Institute for Public Health and the Environment (RIVM), stichting "Stimuleringsfonds Alternatieven voor Dierproeven" and WIL Research is gratefully acknowledged.

ISBN/EAN 978-94-6203-277-4

Cover Layout: Peter T. Theunissen, Nijmegen

Portrait Photo: Lieke van Mil Photography (http://www.liekevanmil.com/) Layout: Promotie in Zicht, Arnhem

Production: CPI / Wöhrmann Print Service, Zutphen

\section{(OPeter T. Theunissen, Nijmegen, 2013}

All rights reserved. No part of this publication may be reproduced or transmitted in any form or by

any means, electronic or mechanical, by print or otherwise, without permission in writing of the author. ter verkrijging van de graad van doctor aan de Universiteit Maastricht, op gezag van de Rector Magnificus, Prof. dr. L.L.G. Soete volgens het besluit van het College van Decanen, in het openbaar te verdedigen

op vrijdag 1 maart 2013 om 12.00 uur

door

Petrus Theodorus Theunissen

Geboren te Nijmegen op 14 april 1983 
Promotores

Prof. dr. J.C.S Kleinjans

Prof. dr A.H. Piersma (Universiteit Utrecht, RIVM)

\section{Copromotor}

Dr. J.L.A. Pennings (RIVM)

\section{Beoordelingscommissie}

Prof. dr. H.W.M. Steinbusch (voorzitter)

Prof. dr. M. van den Berg (Universiteit Utrecht)

Prof. dr. W.H. Lamers

Prof. dr. H. van Loveren

Prof. dr. C.L. Mummery (Leids Universitair Medisch Centrum)

\section{Contents}

Abbreviations..................................6

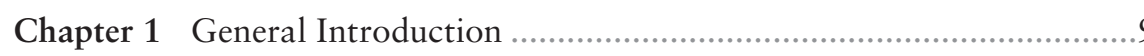

Chapter 2 An abbreviated protocol for multi-lineage neural ..................... 25 differentiation of murine embryonic stem cells and its perturbation by methylmercury

Chapter 3 Time-response evaluation by transcriptomics of .... methylmercury effects on neural differentiation of murine embryonic stem cells

Chapter 4 Transcriptomic concentration-response evaluation of .... Valproic Acid Cyproconazole and Hexaconazole in the neural embryonic stem cell test (ESTn)

Chapter 5 Compound-specific effects of diverse neurodevelopmental ........93 toxicants on global gene expression in the neural embryonic stem cell test (ESTn)

Chapter 6 An optimized gene set for transcriptomics-based neurodevelopmental toxicity prediction in the neural embryonic stem cell test (ESTn)

Chapter 7 Complementary detection of embryotoxic properties of .... substances in the neural and cardiac embryonic stem cell tests (ESTn and ESTc)

Chapter 8 Comparison of methylmercury-induced toxicogenomic responses in in vivo and in vitro models used in developmental toxicology

Chapter 9 Summary and general discussion

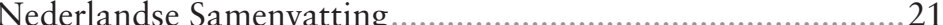

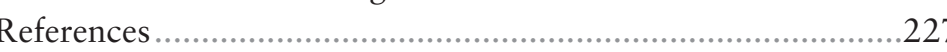
Acknowledgements ..............................................................251

List of Publications and patents ………………ㄴ..

Curriculum Vitae ...............................25 


\section{Abbreviations}

ACE Acetaldehyde

Absolute Fold Ratio

BSA B Bovine Serut Alburin

CBZ Carbamazepine

CDF Chip Description File

Complementary DNA

CM Complete Medium

CON Control Group

CYP Coxicology Database

Cyproconazole

DAVID Database for Annotation, Visualization and Integrated Discovery

DEHP Bis(2-ethylhexyl)phthalate

Deoxyribonucleic Acid

DMEM Dulbecco's Modified Eagle Medium

DMSO Dimethyl Sulfoxide

EB Embryoid Body

ECC Embryo Carcinoma Cells

ECVAM European Centre for the Validation of Alternative Methods

ESC Embryonic Stem Cells

EST Embryonic Stem Cell Test (ECVAM)

ESTc Cardiac Embryonic Stem Cell Test

ESTn Neural Embryonic Stem Cell Test

FACS Fluorescence Activated Cell Sorting

FASD Fetal Alcohol Spectrum Disorder

FDR False Discovery Rate

FC Fold Change

FLU Flusilazole

GD Gestational Day

GO Gene Ontology

GOBP Gene Ontology Biological Process

GSEA Gene Set Enrichment Analysis

HBSS HEPES Buffered Saline Solution

HEX Hexaconazole

Histone Deacetylase

International Conference of Harmonization

Inhibitory Concentration $\mathrm{xx} \%$

Inhibitory Concentration of Differentiation 50\%

Insulin-Transferrin-Selenite-medium

In Vitro Transcription

KEGG Kyoto Encyclopedia of Genes and Genomes

LIF Leukemia Inhibitory Factor

LOOCV Leave One Out Cross Validation

LS Low Serum medium

MEFs Mouse Embryonic Fibroblasts

$\mathrm{MeHg}$ Methylmercury

MEHP Monoethylhexyl Phthalate

Mouse Embryonic Stem Cells

mRNA Messenger Ribonucleic Acid
NCBI National Center for Biotechnology Information

NPC Neural Precursor Cells

NRC National Research Council

NSC Neural Stem Cells

OECD Organization for Economic Co-operation and Development

OECD Organization for Econor

PBS Phosphate-Buffered Saline

Principal Component

PCA

ENG Penicillin $\mathrm{G}$

PHE Phytoin

Poly-L-ornithine

Possible Risk Obtained from Animal Studies

all-trans Retinoic Acid

REACH Registration Authorization and Evaluation of Chemicals

RIN RNA Integrity Number

RMA Robust Multichip Average

RNA Ribonucleic Acid

Receiver Operating Characteristic

RT-PCR Real-time Polymerase chain Reaction

Q)SAR (Quantitative) Structure-Activity Relationship

U.S.FDA United States Food and Drug Administration

VPA Valproic Acid

WEC Whole Embryo Culture

ZET Zebrafish Embryotoxicity 


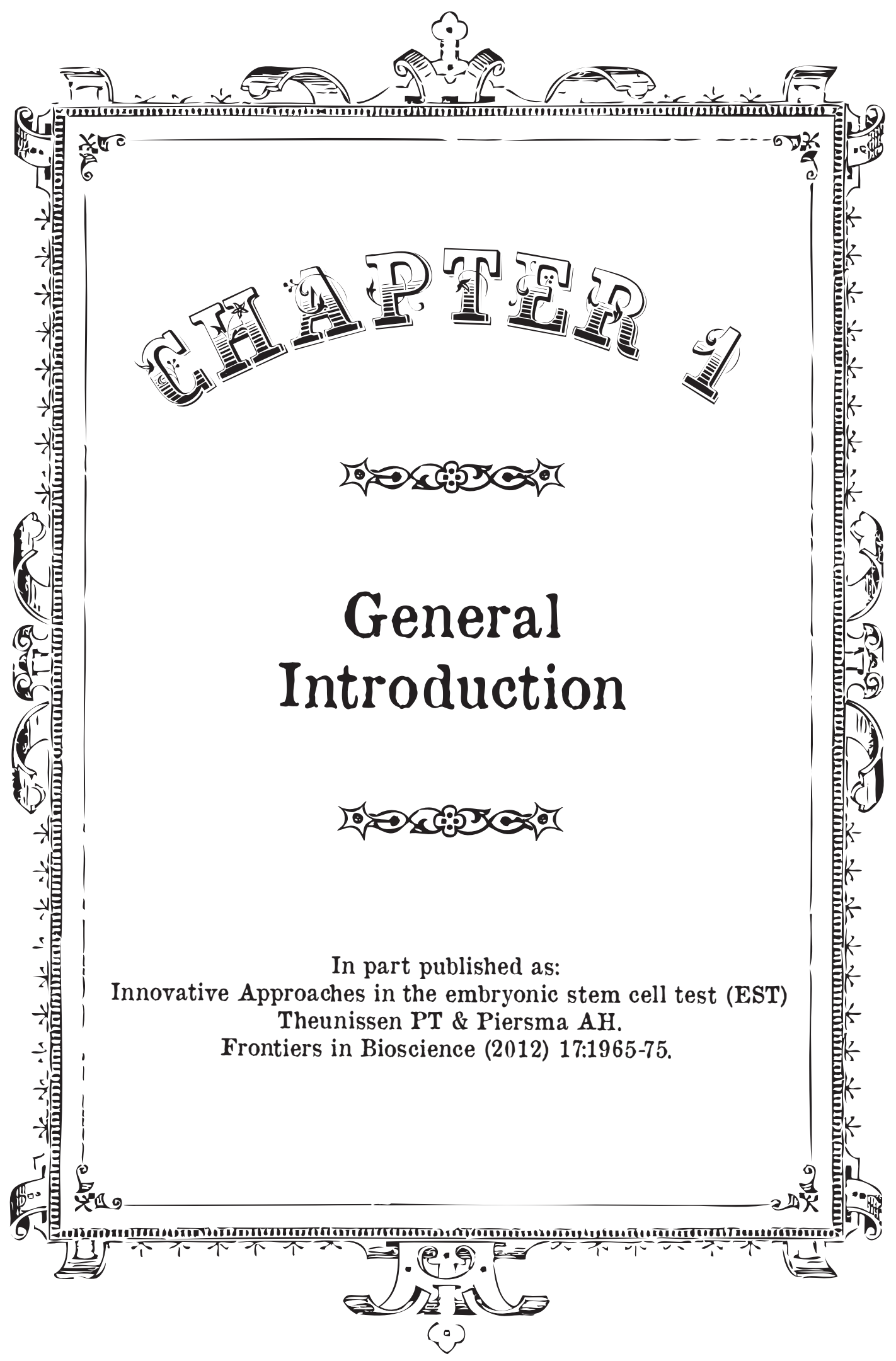




\section{Evolution of developmental toxicity testing}

TRAtology as a modern science has its origins in the early 1930s, when 1 Fred Hale discovered that vitamin A deprivation in food of pregnant pig sows resulted in offspring with developmental defects, including ocular defects (anophthalmia) and cleft palate [1]. Subsequent studies on vitamin A deprivation during pregnancy in other species confirmed the importance of vitamin A as a morphogen during embryogenesis [2]. In addition, a few years later, it was demonstrated that excess vitamin A consumption by pregnant rats as well induced malformations in offspring, indicating the balance of vitamin A intake is critical during development [3]. In the following two decades, the importance of disease and chemical induced developmental defects in humans became evident, including the correlation between maternal rubella infection and children born with birth defects and environmental methylmercury $(\mathrm{MeHg}$ poisoning inducing (neuro-)developmental defects, a syndrome at presen characterized as congenital Minamata disease [4, 5]. It was not until the late 1950s and early 1960s, when the thalidomide tragedy was laid bare, that the importance of developmental toxicity testing of pharmaceuticals and chemicals became evident [6]. The presumed safe drug thalidomide was prescribed to pregnant women as a mild sedative, however unexpectedly, exposure during pregnancy caused severe congenital malformations to the developing embryo in more than 10.000 women [6]. Concurrently, in 1959 Wilson proposed the six general principles of teratology, which today still are valid largely unchanged and important aspects in current advancement of teratological studies [7]. These principles included topics such as genotype and adverse environmental factors interactions, varying susceptibility for developing malformations to a compound over developmental stages, multiple mechanisms of action between compounds resulting in comparable morphological endpoints, dose-response relationships and different manifestations of deviant development (death, malformation, growth retardation and functional defect). Due to the thalidomide incident, the United States Food and Drug Administration (U.S FDA) issued extensive in vivo animal testing protocols for reproductive and developmental toxicity, based on the Wilson's principles of teratology [8] These protocols were eventually incorporated in worldwide guidelines for developmental toxicity testing of chemicals in the 1980s by the Organization or Economic Co-operation and Development (OECD) and for pharmaceuticals in the International Conference of Harmonization of Technica Requirements for Registration of Pharmaceuticals for Human Use ( $\mathrm{ICH}$ ) [9-11]. These guidelines were designed to cover all critical stages during reproduction and development, from mating and conception to gestation, 
prenatal development and postnatal development. In 2009, the new European legislation for Registration, Evaluation, Authorization and Restriction of Chemicals (REACH) came into force, which required all compounds produced and used in the European Union to be tested for toxicity, including reproductive and developmental toxicity $[11,12]$. Testing protocols required within REACH are mainly based on animal testing strategies, with relatively few allowed in vitro alternatives. However, development of in vitro alternatives for toxicity testing was greatly supported in the REACH legislation. Early calculations on the amount of experimental animals needed within REACH predicted that approximately $65 \%$ of the animals used will be accounted for reproductive and developmental toxicity testing, with a predicted cost between 1.3 and 9.6 billion euro $[12,13]$. Due to the high costs of the time consuming animal test protocols and the increasing public ethical awareness to reduce animal testing, it became highly desirable to develop high-throughput alternative screening methods for toxicity testing, especially in the field of reproductive and developmental toxicology.

\section{In vitro developmental toxicity testing}

In order to reduce the number of animals needed for developmental toxicity testing and screening, a range of in vitro test systems have been developed over the past decades, of which the most promising test systems include the rat whole embryo culture (WEC), the zebrafish embryotoxicity test (ZET) and the murine embryonic stem cell test (EST) [14-17]. In the WEC and the ZET systems compound developmental toxic properties are assessed on live whole embryos derived from pregnant rats or fertilized zebrafish eggs, and are therefore not completely animal free alternative test systems, although the number of animals used for these test systems is predicted to be 10-100 fold lower. A potential high-throughput in vitro screening assay for developmental toxicity free of animal use is the EST, first described in 1997 by Spielmann $e$ al. [18]. This test system is based on the ability of blastocyst-derived pluripotent murine embryonic stem cells (ESC) to differentiate into virtually any cell type present in the vertebrate organism, such as cardiomyocytes, neurons and osteoblasts [19]. In the original EST, differentiation of the ESC towards cardiomyocytes is induced through the hanging drop culture technique [20], in which the ESC form aggregates called embryoid bodies (EB) [21]. EBs resemble the anterior pre-streak embryo, with the epiblast-like core able to generate derivatives of all three primary germ layers; mesoderm, ectoderm and endoderm [19]. In the EST, cardiomyocyte differentiation is further induced by growing the EB in suspension culture for two days after which EB are plated on plastic tissue culture dishes. Cells of the EB further migrate and differentiate and form a plaque of heterogeneous cell types, including contracting cardiomyocytes, which can be observed through the microscope. Compound inhibition of ESC differentiation into cardiomyocytes in this system is regarded as a measure for developmental toxicity.

\section{Intermezzo - a short history of stem cell research}

The term 'stem cell' was first proposed by the Russian histologist Alexander Maximow in 1909 for cell types present during development and postnatal in bone marrow, currently defined as haemapoietic stem cells [22]. More than fifty years later, McCullough and Till demonstrated the presence of self-renewing cells in mouse bone-marrow, which they later defined as stem cells [23, 24]. In 1964, the pluripotency of single cells derived from an embryocarcinoma was shown, ultimately resulting in the first established embryocarcinoma cell (ECC) lines in the early 1970s [25,26]. Due to their pluripoten nature, ECC could be differentiated into a wide variety of cell types, providing insight in early differentiation in the vertebrate embryo [27, 28]. Subsequently, ECC systems were used to study perturbation of differentiation by developmental toxic compounds [29]. However, due to the nature of ECC as cancer cells, they have many limitations and imperfections, including chromosomal aberrations, a chance to lose the ability to differentiate and the need of addition of chemical inducers to initiate differentiation in vitro [30]. In 1981, two research groups separately isolated the first murine ESC derived from the inner cell mass of the blastocyst, followed by isolation of human ESCs in 1998 [31-33]. ESC, being more close to cell differentiation in vivo compared to ECC, exhibit less of the problems associated with ECC culture and differentiation, and over time, have become the default cells for studying cell differentiation in vitro and currently form the basis for studying compound effects on differentiation in the EST.

\section{Validation of the (cardiac) EST}

In order to optimize the EST test protocol, a pre-validation study was performed in which ten compounds inducing developmental toxicity in vivo with diverse potencies were tested by two laboratories [34]. In this study, the prediction model described earlier by Spielmann et al. [18] misclassified four out of ten compounds. An improved statistical prediction model was then introduced, which resulted in an accuracy of $93 \%$ for the same test compounds [34].

Following the pre-validation study, a larger validation study, funded by the European Centre for Validation of Alternative Methods (ECVAM), was performed following the ECVAM approach for validation of alternative methods $[17,35]$. This validation study was set up as an inter-laboratory blind 
trial in which four laboratories of different international institutes tested 20 compounds, each with a different in vivo developmental toxicity potency, without the need of metabolization (non-developmental toxic, weakly developmenta toxic and 7 strongly developmental toxic). The improved prediction mode predicted the developmental toxicity of the compounds with an overal accuracy of $78 \%$. For the strong developmental toxicants, prediction was very good $(100 \%)$ with a precision of $86 \%$. Prediction was defined as the likelihood that a positive prediction in EST correctly identifies a strongly developmental toxic compounds. Precision was defined as the proportion of compounds which are strongly developmental toxic in vivo and correctly classified as such in the EST. The only strong developmental toxic compound not correctly predicted by the prediction model was the neurodevelopmental toxicant $\mathrm{MeHg}$, which was classified as non-developmental toxic in 4 out of 8 experiments. Predictivity of the weak- and non-developmental toxic compounds was much lower $169 \%$ and $73 \%$, respectively) producing a relatively high rate of false positive classifications. The test system was therefore deemed good in determining strong developmental toxicants, but the tests limitation lied in discriminating between weak and non-developmental toxicants [17]. The first validation test was very promising for the implementation of the EST as a test system to screen for developmental toxicants, however, it was based on a limited set of compounds, and further characterization with additional compounds and optimization of the model on the basis of results was deemed necessary.

In 2003, ECVAM organized an expert workshop to determine the applicability domain of the EST [36]. Furthermore, it was evaluated if the validated EST could be used as a screening test for lead compounds in the pharmaceutical industry and to detect the developmental toxicity of compounds in the chemical industry under REACH. Due to a number of limitations in the test system [36] the EST in its current form was not deemed fit to be accepted in regulatory context, but could be used to add supportive information. Furthermore, it was recommended that, to optimize the predictability and increase knowledge on the applicability domain of the EST, a number of improvements should be added to the EST, including:

1) the existing chemicals database should be expanded with known in vivo embryotoxicants,

2) differentiation of additional specific lineages should be incorporated in the testing paradigm (e.g. neural differentiation, osteoblast differentiation, etc.),

3) objective quantitative endpoints should be established,

4) in vitro / in vivo concentration correlations should be considered,

5) additional (statistical) prediction models should be developed,
6) a metabolic activation system should be incorporated,

7) the stability of the test compounds should be assessed.

As a response to the recommendations in this workshop, a selection procedure was performed by ECVAM and international experts, resulting in a group of thirty-one compounds which were selected to be included in a second screening study. Thirteen of these compounds, strong, moderate or non-developmenta toxic in vivo, were tested by two laboratories in the European ReProTect Consortium [37]. The analysis for these compounds demonstrated only 2 out of 13 compounds were classified correctly, indicating the improved prediction model was not capable of predicting developmental toxicity for this compound set. In a workshop organized by the EU $6^{\text {th }}$ framework programme ReProTect project and ECVAM, the unexpected results were discussed, and recommendations to modify the test system were advised [37]. These again included modifications to the EST protocol, addition of a metabolic system and new differentiation endpoints to be studied using molecular markers.

In recent years, as an answer to the recommended improvements to the EST, many proposals have been published to optimize, shorten and enhance the cardiac EST regarding predictivity, objective molecular endpoint parameter and technical improvements [37-40]. In addition, novel differentiation endpoints have been proposed $[36,37,41,42]$. In the following sections, in vitro neura differentiation and the use of molecular endpoints will be further addressed as improvements for the EST.

Neural differentiation models in neurodevelopmental toxicity testing Although compounds are known which induce subtle neurodevelopmental and behavioral effects to offspring at lower doses than in the adult, until recently at the regulatory level, compound and pharmaceutical induced effects on neurodevelopment and behavior were relatively under-investigated [43-45]. For example, under OECD testing guidelines, compound induced effects on neura development were only assessed at the histological and malformation level in the fetus (OECD guidelines 414, 416 and 421) [46]. Recently, OECD introduced new guidelines to predict neurodevelopmental toxicity: the developmenta neurotoxicity study (OECD 426) and the extended one generation study, ncluding a developmental neurotoxicity cohort (OECD 443). Following these guidelines, both central nervous system histology as well as behavior are to be assessed. In order to screen for potential compound induced effects on neurodevelopment, in vitro screening assays are required to reduce animal testing, cost and time and to gain mechanistic insight in compound induced neurodevelopmental toxicity. 
One of the first in vitro systems ever developed was based on differentiation of neuroblastoma cells. In 1907, the embryologist Ross G. Harrison for the first time successfully cultured neuroblasts from Xenopus laevis to study nerve ending growth, taking the first step into current in vitro precursor cell and stem cell research [47, 48]. Furthermore, in 1967, Joseph Altman observed neurogenesis due to stem cell differentiation in the adult brain, contradicting the then believed paradigm of a non-growing, fixed brain structure [49]. However, his research was not widely accepted and forgotten over time, only to be 're-discovered' in the early 1990's leading into exceptionally increased research in the field of neural differentiation and brain plasticity. Due to this increased interest, over the last few decades many in vitro neural cell lines have been developed to study neural development and neuron differentiation, including PC12 cells, embryonal precursor cells derived from a tumor which can be differentiated to form neurites, neuroblastoma cells, cells derived from a neuroblastoma cancer, neurospheres, derived from embryonic neura progenitor cells, and most recently both mouse and human ESC neural differentiation systems [50-55]. The first mouse ESC based neural differentiation protocols were described in the early-1990's and many different protocols for neuron and glia cell differentiation have been published since $[52,56]$. In order to differentiate mouse ESC into neural cells, most neural differentiation protocols depend on EB formation, addition of the morphogen all-trans retinoic acid (RA), serum deprivation and/or addition of neural growth factors [56]. Although these protocols are relatively laborious, the advantage compared to other available in vitro models is that all stages of neural development can be studied: from ESC to neural stem cells to precursor cells to ultimately mature neurons and glia [19]. Furthermore, ESC obtained from the inner cell mass of the blastocyst are not immortalized or derived from a tumor, retaining the in vivo quality of these cell types. However, in order to make an ESC-based neural differentiation protocol suitable for neurodevelopmental toxicity testing, it has to be standardized, characterized, validated and the applicability domain should be assessed [57].

In vivo, development of the nervous system is an intricate dynamic process, requiring coordinated expression of a range of complicated cellular and molecular events in a temporal- and region dependent process [44, 58]. Cell types and key processes important in neural development are proliferation, differentiation of precursor cells into neurons and glia cells, migration, growth of axons and dendrites, synapse formation, myelination and programmed cel death [59]. To incorporate all these endpoints in one in vitro system in order to study neurodevelopmental toxicity is highly complicated, therefore a battery of test systems, each describing a sub-process in neural development is needed. This complexity has resulted in a range of diverse test systems published, studying compound perturbation on different aspects of neural developmen [60]. The main focus currently rests on morphological endpoints, such as effects on axonal outgrowth and determining molecular endpoints predicting perturbation of neural differentiation [61]. Due to the complexity of neura differentiation, it is advisable to first characterize the culture systems and then study the mechanisms behind neurodevelopmental toxicity in these models in order to define the applicability domain of these neural differentiation assays. For example, with the aim of gaining insight in neural differentiation systems, Kuegler et al. [62] investigated critical processes mediating mouse ESC differentiation into cells of the nervous system. A list of in total 197 mRNA marker was compiled from literature defining undifferentiated mouse ESC, neura stem cells, astrocytes and the pattern of different neuronal and non-neurona cells generated. These mRNA markers could be supportive in determining neurodevelopmental toxicity in neural differentiation cultures. However, to fully understand mechanisms of neurodevelopmental toxicity, further molecular characterization for each developed model should be performed. A relatively new tool to do this is genomics.

\section{Transcriptomics as a tool for predictive toxicology}

Recent technological advancements in molecular biology introduced new tools to investigate thousands of molecular endpoints in a single assay. This technology, called genomics, has its origins in the discovery of the DNA structure by Watson and Crick in 1953 nearly 60 years ago [63]. In the following 60 years, translation of the DNA code to function in the cell has substantially progressed, revealing molecular information on signaling and functional processes within the cells of an organism. Together with our increasing knowledge of these molecular processes, detection methods have developed from single gene or molecule detection to rapid and high throughput on-chip systems with which expression of thousands different genes or molecules can be assessed [64]. This technology in combination with the unraveling of the genome sequence of multiple species, including the human, made it possible to study compound effects genome-wide in one experimen [65]. Currently available state-of-the-art techniques can be used to study the whole transcriptome (RNA transcripts of DNA), proteome, epigenome, metabolome or other '-omes'. Transcriptomics approaches can be used to increase knowledge on biological processes in the fields of physiology, pathology and toxicology. The application of genomics technologies in toxicology provided the new research field toxicogenomics [66]. Toxicogenomic 
profiling has been applied to identify molecular mechanisms behind toxicity, to search for biomarkers to identify toxicity and may be used to identify compound-class specific induced toxicity effects. The first toxicogenomic studies were performed in the field of carcinogenesis, where in vivo effects of known genotoxic and non-genotoxic carcinogens on transcription in the liver was assessed and later could be discriminated between the two classes $[67,68]$ Next to prediction of genotoxic-carcinogenicity, toxicogenomics has been used to predict other toxic responses, including immunotoxicity, developmental toxicity, reproductive toxicity and endocrine disruption, among others, and is occasionally used in drug discovery and development to study adverse drug effects [69-72]. In vivo developmental and reproductive toxicity studies using toxicogenomics as a readout have improved knowledge on understanding processes involved with specific compound perturbed developmental toxicity, including endocrine disruptors, metals, anti-fungicides (triazoles), vitamin A and anticonvulsants [73-77]. In order to predict developmental toxicity in a high throughput fashion, more recent studies used in vitro methods for prediction of known and possible developmental toxicants, including the WEC, ZET and the cardiac EST (ESTc) [78-82]. In one of the first of these studies, gene expression profiling in the WEC showed distinct and relevant alterations in gene expression after compound exposure at a time poin preceding morphologically detectable developmental toxic responses, indicating a transcriptomics-based method can be used to predict developmental toxicity earlier in time [82]. Transcriptomics techniques have additionally been used to further characterize and validate the ESTc. By studying multiple compound effects at an earlier time point, van Dartel et al. were able to develop gene sets specific for early cardiomyocyte related mesoderm differentiation and ultimately introduce a biomarker gene set to predict developmental toxicity effects on cardiomyocyte differentiation [83, 84].

\section{Analysis of transcriptomics data}

Transcriptomics experiments generate massive amounts of data, providing a challenge for data analysis. For instance, the mouse whole genome Affymetrix array chips used for experiments in this thesis consist of 39.000+ transcripts, which after a correction for non-annotated genes, ultimately represent more than 16.000 genes as data points per sample. In order to analyze and interpre differences between control samples and treated samples analysis strategies and tools have been developed.

A standard first approach is to identify the differentially expressed genes, being the genes regulated by exposure compared to control. Selection of these genes is defined by a significance cutoff value, which can be a $p$-value or a false discovery rate (FDR) [85]. Furthermore, as an additional cutoff the fold change (FC) can be used, which takes into account the level of difference in gene expression between the exposed sample and the control. Genes differentially expressed can then be visualized for further interpretation using cluste analysis, such as hierarchical clustering or principal component analysis (PCA) $[86,87]$. These tools can be used to visualize similar or dissimilar gene expression patterns for single genes or groups of similarly behaving genes [88] When studying single genes, biological function and other information can be manually assessed using databases collecting scientific literature on single genes, such as National Center for Biotechnology Information (NCBI), GeneCards (the human genome compendium), Gene Ontology (GO) and the Comparative Toxicogenomics Database (CTD) [89-91].

When interested in a shared biological function of a set of genes, overrepresentation of these genes annotated to biological processes, molecular function (GO) or pathways (KEGG- or Wiki-pathway) can be assessed using the Database for Annotation, Visualization and Integrated Discovery (DAVID) or GO-Quant, which additionally provides a quantitative average ratio of the genes expressed within the biological process or molecular function [92-95] These analyses will provide additional information on gene set shared biology, function or cellular location, but will not provide information on gene interaction within the set. In order to study the interconnection of the genes within a set, pathway analysis can be performed using the publicly available STRING, STITCH, Wiki pathways, GenMAPP and KEGG, or the commercial available MetaCore and Ingenuity Pathway Analysis Tool [96-98]. However, due to the limited knowledge on development related pathways, use of these program is of less additional value in research for developmental toxicity.

Previous described tools all make use of a predefined set of genes. Another way to analyze data is by making use of all the available data and determine average gene changes of genes in biological processes or molecular function terms, by using Tox-profiler or Gene Set Enrichment Analysis (GSEA) [99, 100]. This approach can provide additional information on gene set involvement in processes by detecting subtle and coordinated expression changes over the whole set. Although this approach does not provide additional information on biological function, it does allow detection of regulated genes in predefined processes of which individual genes may not meet significance threshold criteria. 


\section{Objective and outline of this thesis}

\section{Objective of the thesis}

The classic EST was developed as a response to the high need of in vitro alternatives in developmental toxicity testing. After validation of the EST, in order to increase predictivity of the assay, it was proposed to build in modifications to the test system. These included incorporation of additional differentiation routes to the testing paradigm to reduce the number of misclassified compounds and implementation of molecular techniques as objective endpoints. Incorporation of a neural embryonic stem cell test (ESTn) in the EST testing paradigm is assumed to provide additional information to specific neurodevelopmental toxicants which were misclassified in the EST, such as $\mathrm{MeHg}$ [17]. In addition, due to increased knowledge in the field of neurodevelopmental toxicity, there is a growing demand for in vitro alternatives in this field.

Use of genomic technologies in toxicology can provide mechanistic insight in toxicity and test system applicability domain. Furthermore genomic technologies may ultimately provide gene set biomarkers for prediction of specific modes of toxicity. Implementation of genomics technologies in a neural variant of the EST may therefore enhance knowledge of the mechanisms behind neura development and compound perturbation of development.

The aim of this research is to develop a high-throughput alternative in vitro assay for prediction of neurodevelopmental toxicity, of which the method is similar to the EST for easy inter-model comparisons. In addition, transcriptomics techniques will be used to optimize and characterize the culture system and to assess compound induced mechanistic effects on early neural development.

Ultimately, combined analysis of multiple compound exposure studies should lead to discovery of a biomarker gene set which is able to predict developmental toxicity in the ESTn.

A schematic overview of the experiments described in each chapter of this thesis is provided in Figure 1. Incorporation of the ESTn with molecula endpoints in the EST testing paradigm may contribute to increase mechanistic insight in compound induced (neuro)developmental toxicity and reduction of animal testing in this field.
Test System Variables

Analysis method

Chapter 2
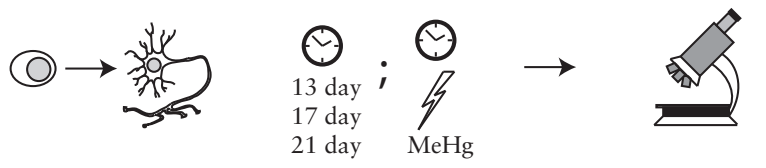

Chapter 3
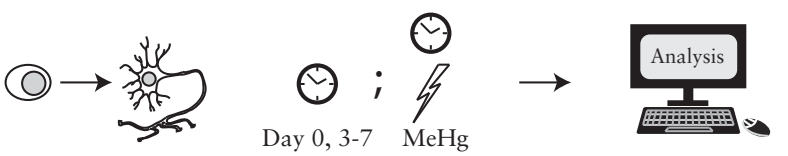

Chapter 4
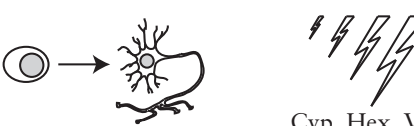

Cyp, Hex, VPA

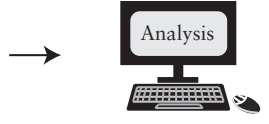

Chapter 5
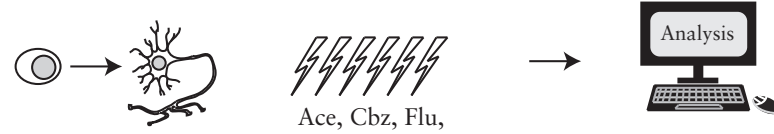

Ace, Cbz, Flu,
MEHP, PenG, Phe

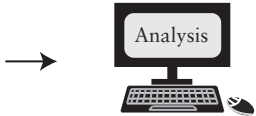

Data Chapter 3, 4,

Chapter 6
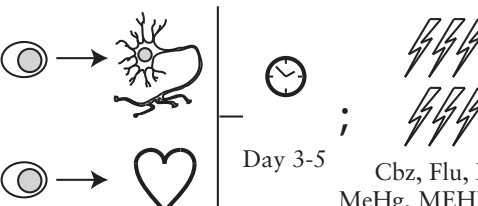

Chapter 7

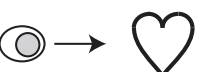
Cbz, $\mathrm{MeHg}, \mathrm{MEHP}, \mathrm{PenG}$
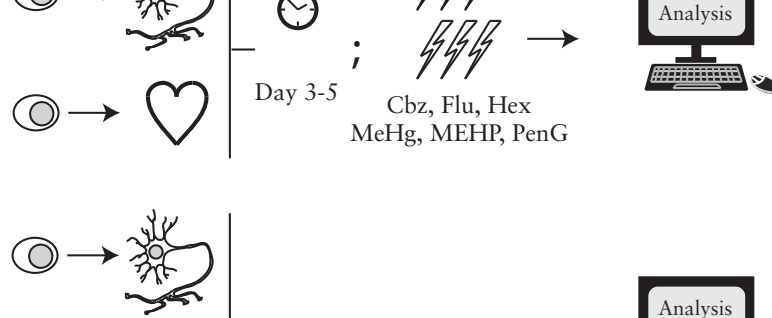

Chapter 8

(2)

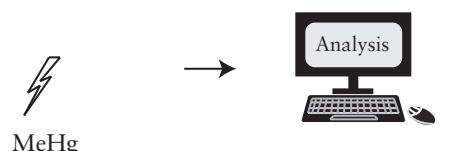

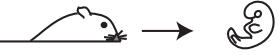

Figure 1 Schematic overview of the experiments described in this thesis 


\section{Outline of the thesis}

In order to increase prediction of the classical EST, additional differentiation end points have been proposed. In chapter 2 the development of a murine ESC neural differentiation test system for assessment of neurodevelopmental toxicity is described. Three protocol lengths (13, 17 and 21 days) are characterized over time and compared, leading into a shortened EST-resembling neural differentiation protocol, called the neural embryonic stem cell tes (ESTn). As a proof-of-principle, the effects of the model neurodevelopmental toxicant methylmercury $(\mathrm{MeHg})$ are assessed in the ESTn by microscopic morphological scoring of neural outgrowth. Three exposure designs were performed to determine the most optimal exposure conditions. The developed morphological scoring system during these experiments was used to determine compound induced effects on neural outgrowth in following studies.

A transcriptomics approach was used in chapter 3 to further mechanistically characterize neural differentiation over time in the ESTn. Samples were taken at the start of the protocol (day 0) and at consecutive days 3, 4, 5, 6 and 7 and specific time-dependent changes following neural differentiation could be described. Furthermore, as proof-of-concept $\mathrm{MeHg}$ induced changes on gene expression were assessed over time after 24, 48, 72 and 96h exposure starting from day 3 onwards. Based on these data an exposure of $24 \mathrm{~h}$ was chosen for future experiments.

Next to time, concentration is also an essential variable for determining compound toxicity [101]. In chapter 4 compound induced concentrationdependent effects on neural differentiation after $24 \mathrm{~h}$ exposure are assessed at four concentrations for three known developmental toxic compounds; the triazoles cyproconazole and hexaconazole and the anticonvulsant valproic acid. Differences and similarities in gene expression profiles and process enrichment between compounds and compound classes are described.

To further determine the applicability domain of the ESTn, in chapter 5 transcriptomics study was performed in the ESTn to determine the effects of six mechanistically diverse compounds at two concentrations, one morphologically non-toxic and one 'developmental' toxic but not cytotoxic. Five of the studied compounds were known developmental toxicants (acetaldehyde, carbamazepine, flusilazole, monoethylhexyl phthalate and phenytoin) and one was a negative control (penicillin G). In addition, assessment of compound induced gene expression at the low non-toxic concentration and the high toxic concentration was assessed to study adaptive and adverse gene expression responses to compounds.

In chapter 6 data from chapters 3,4 and 5 is used to perform a de novo analysis on combined raw data (10 compounds, 19 exposures) to identify an optimized gene set for neurodevelopmental toxicity prediction in the ESTn. The PCA based differentiation track algorithm was used to sample random gene sets for identification of the best performing prediction.

The ESTn was designed as a companion test system to the cardiac EST (ESTc), to improve prediction and mechanistic insight in compound (neuro) developmental toxicity. To confirm this, it was needed to investigate similar and dissimilar characteristics of both methods at the transcriptome level. In Chapter 7 time-induced gene expression changes in ESTn and ESTc are compared over days 3, 4 and 5 in both models. Furthermore, compound induced changes after $24 \mathrm{~h}$ exposure of seven compounds are compared between the two models.

In order to be able to perform correct extrapolation in risk assessment for in vitro models, it is mandatory to know the similarities and differences between in vitro and in vivo models. In chapter 8 toxicogenomic comparisons are made between in vitro and in vivo models for developmental toxicity and general toxicity after exposure to MeHg. Developmental toxicity models used in this ssessment are the in vitro ESTn, ESTc and WEC and two in vivo rat embryotoxicity tests. General toxicity models used are mouse embryonic fibroblasts in vitro and transcriptomics on adult rat brain and liver in vivo.

Chapter 9 summarizes the results of all previous chapters and provides a general discussion on the development and implementation of transcriptomics techniques in the ESTn. Furthermore, possible implementation of the ESTn in the current testing paradigms will be discussed.

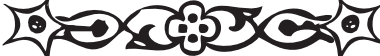




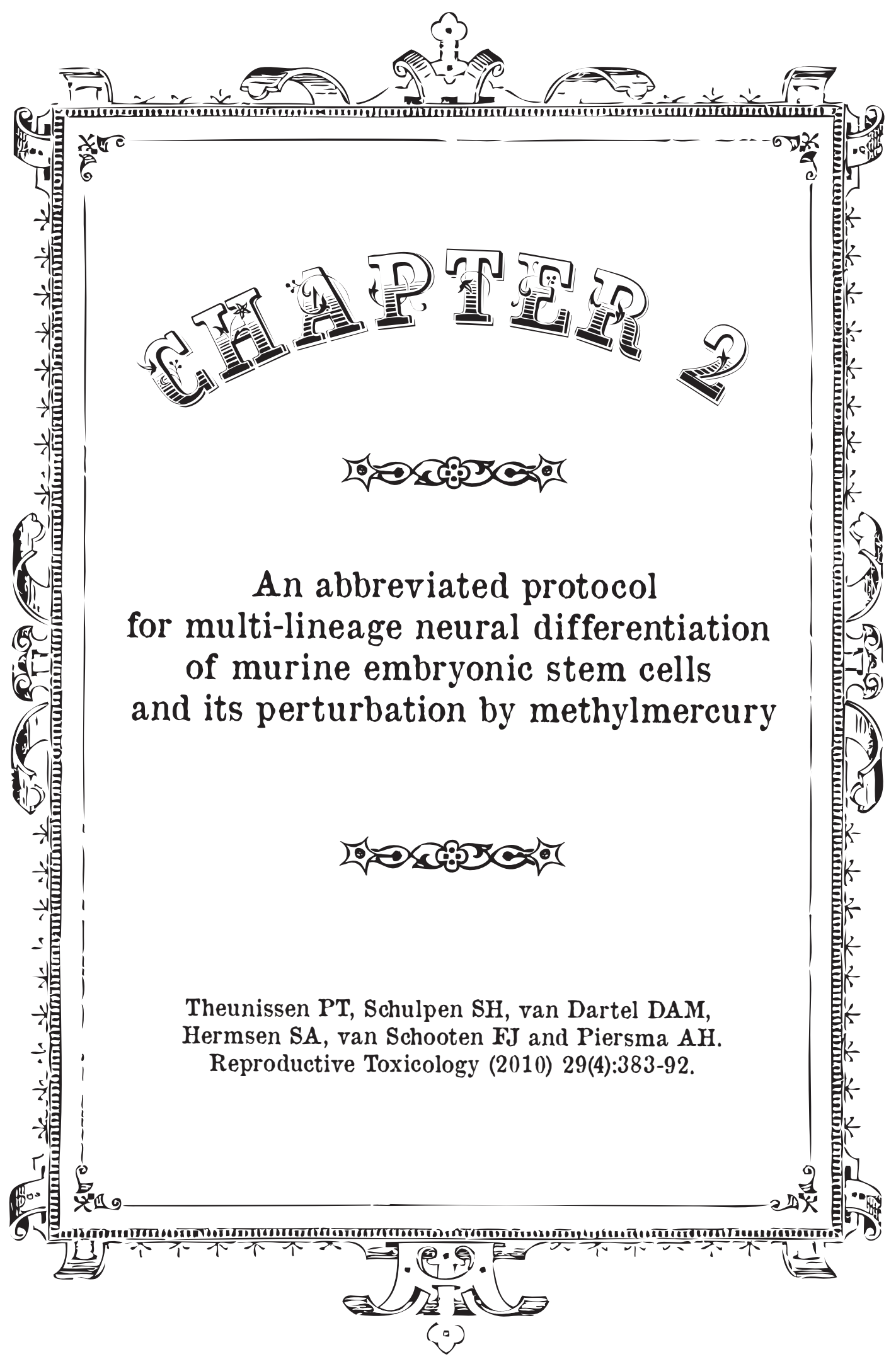




\section{Abstract}

Lternative assays are highly desirable to reduce the extensive experimental A animal use in developmental toxicity testing. In the present study, we developed an improved test system for assessing neurodevelopmental toxicity using differentiating mouse embryonic stem cells. We advanced previously established methods by merging, modifying and abbreviating the original 20-day protocol into a more efficient 13-day neural differentiation protocol. Using morphological observation, immunocytochemistry, gene expression and flow cytometry, it was shown predominantly multiple lineages of neuro-ectodermal cells were formed in our protocol, and to a lower extent endodermal and mesodermal differentiated cell types. This abbreviated protocol should lead to an advanced screening method using morphology in combination with selected differentiation markers aimed at predicting neurodevelopmental toxicity. Finally, the assay was shown to express differential sensitivity to a model developmental neurotoxicant, methylmercury.

\section{๕̊ำ}

\section{Introduction}

C hemical risk assessment is currently still highly dependent on globally harmonized experimental animal studies. Reproductive toxicity testing proposed within the European chemical safety legislation (REACH) has been estimated to require approximately $60 \%$ of the total animals needed for toxicity testing $[12,102]$. Therefore, this area of toxicity testing is a high priority area for designing alternative assays to reduce experimental anima use. An established in vitro test system for developmental toxicity is the embryonic stem cell test (EST) [17], in which the developmental toxic effect of compounds on stem cell differentiation towards beating cardiomyocytes is assessed. Despite the promise of this in vitro developmental methodology, a recent validation study suggests a number of in vivo developmental toxic compounds are misclassified as negative in the EST, one of which being methylmercury chloride [17]. Misclassifications may be partly due to the prediction model used, in which both proliferation and differentiation parameters play a role. However, these compounds may have been misclassified, because they may not affect mesodermal-derived cardiomyocyte differentiation in vivo, but primarily affect alternative differentiation routes, such as the ectodermal or the endodermal routes [17]. Additional alternative in vitro embryonic stem cell differentiation assays complementary to the EST may improve the prediction for such compounds.

Several neural differentiation protocols for murine embryonic stem cells (ESC) have been described since the mid 1990s, most of which were developed for mechanistic studies of embryonic cell differentiation [52, 54]. These methods use a variety of factors to reach a similar extent of neural differentiation. They include retinoic acid (RA) [54, 103], serum deprivation [52], hormones and growth factors and supporting matrices for a range of different neural end points [104]. Most methods make use of three-dimensional embryoid body (EB) formation [52, 54], while some methods use a two-dimensional monolayer culture [105, 106], or a combination of these two [103]. For the rapid prediction of neurodevelopmental toxicity, it is urgently needed to develop a short duration, high-throughput model, which sufficiently mimics the in vivo differentiation of ESC towards neuronal-type cells. In the present study we have designed a testing model based on methods described by Okabe et al. (1996) [52] and Bibel et al. (2004) [103] using EB formation and stimulation of neura differentiation using RA and serum deprivation. These models appear to approach the in vivo situation in terms of their neural differentiation pattern. However, we have reduced the length of a combination of these two protocols to enable increased throughput of compound testing. Furthermore, multi- 
lineage (ectodermal, mesodermal and endodermal) differentiation of the combined protocols was studied.

In vivo studies have shown that the development of the brain is a proces sensitive towards developmental toxic challenges [45, 107, 108]. During neura system development of the embryo, stem cells differentiate into many types of neurons, glial cells and neuronal epithelial cells [30]. In this highly tuned process, interaction between specific cell types is essential for proper differentiation and the establishment of optimal ratios of cell types in the brain in time and space. It is known from in vivo studies that neurodevelopmental toxicants, such as methylmercury ( $\mathrm{MeHg}$ ) [109] and ethanol [110], can influence these ratios and disrupt the developing brain. Therefore, improvement of assessing the putative effects of neurodevelopmental toxicants is expected by monitoring over time the varying quantities of specific neural cell types using a battery of differentiation markers.

In this study, we used $\mathrm{MeHg}$ to evaluate the responsiveness of our assay. Two well known incidents of $\mathrm{MeHg}$ poisoning occurred in the early 1950s at the Minamata Bay in Japan and 1970s in Iraq. In Japan, due to environmental poisoning more than 21.000 individuals claimed to be affected by $\mathrm{MeH}$ poisoning [109]. Pregnant women who manifested mild or no symptoms were reported to give birth to infants with severe developmental disabilities, including cerebral palsy, mental retardation, and seizures [109]. In Iraq, pregnant women were exposed to $\mathrm{MeHg}$ by dietary intake and a total of 83 women participated in a developmental assessment of their offspring. Results suggested a dose-response relationship associated with delayed neurodevelopmental milestones [111]. Today, $\mathrm{MeHg}$ is widely used as a model neurodevelopmental toxic compound.

In the present study we have successfully designed a 13-day differentiation protocol, in which multiple lineages of neural and other brain-associated cells are formed. In addition, we developed a screening method using a group of differentiation markers, which may be used to predict neurodevelopmenta toxicity. Finally, the model was shown to have differential sensitivity to a developmental neurotoxicant, $\mathrm{MeHg}$. This work represents the first steps towards an assay for assessing developmental neurotoxicity in vitro.

\section{Materials and Methods}

\section{Culture Media}

Complete medium (CM) contained Dulbecco's modified eagle's medium DMEM) medium (Gibco BRL, Gaithersburg, MD, USA) supplemented with $20 \%$ fetal bovine serum (Hyclone, Logan, UT, USA), 1\% nonessential amino acids (Gibco BRL, Gaithersburg, MD, USA), 1\% penicillin/streptomycin Gibco BRL, Gaithersburg, MD, USA), 2 mM L-glutamine (Gibco BRL, Gaithersburg, MD, USA) and $0.1 \mathrm{mM} \beta$-mercapto-ethanol (Sigma-Aldrich, Zwijndrecht, The Netherlands). Low serum medium (LS), had the same composition as CM except that the serum percentage is $10 \%$. Insulin-transferrin-selenite-fibronectin medium (ITS) contained DMEM/Ham's nutrien mixture F12 (DMEM/F12) medium (Gibco, BRL, Gaithersburg, MD, USA) supplemented with $0.2 \mathrm{\mu g} / \mathrm{ml}$ bovine insulin (Sigma-Aldrich, Zwijndrecht, The Netherlands), 1\% Penicillin/streptomycin (Gibco BRL, Gaithersburg, MD USA), $2 \mathrm{mM}$ L-glutamine (Gibco BRL, Gaithersburg, MD, USA), $30 \mathrm{nM}$ sodium selenite (Sigma-Aldrich, Zwijndrecht, The Netherlands), $50 \mu \mathrm{g} / \mathrm{m}$ apo-transferrin (Sigma-Aldrich, Zwijndrecht, The Netherlands) and $2.5 \mathrm{\mu g} / \mathrm{ml}$ fibronectin (Invitrogen, Carlsbad, CA, USA). N2 medium contained DMEM/ F12 medium (Gibco, BRL, Gaithersburg, MD, USA) supplemented with 0.2 $\mu \mathrm{g} / \mathrm{ml}$ bovine insulin (Sigma-Aldrich, Zwijndrecht, The Netherlands), 1\% Penicillin/streptomycin (Gibco BRL, Gaithersburg, MD, USA), $30 \mathrm{nM}$ sodium selenite (Sigma-Aldrich, Zwijndrecht, The Netherlands), $50 \mathrm{\mu g} / \mathrm{ml}$ apo-transferrin (Sigma-Aldrich, Zwijndrecht, The Netherlands), $20 \mathrm{nM}$ progesteron (Sigma-Aldrich, Zwijndrecht, The Netherlands) and $100 \mu \mathrm{M}$ putrescine (Sigma-Aldrich, Zwijndrecht, The Netherlands).

\section{Embryonic Stem Cell Culture}

Murine embryonic stem cells (ESC) (ES-D3, ATCC, Rockville, MD, USA) were routinely sub-cultured every 2-3 days and grown as a monolayer in $\mathrm{CM}$ supplemented with leukemia inhibiting factor (LIF) (Chemicon, Temecula, CA, USA) at a final concentration of 1000 units $/ \mathrm{ml}$. The cells were maintained in a humidified atmosphere at $37^{\circ} \mathrm{C}$ and $5 \% \mathrm{CO}_{2}$

\section{Neural Differentiation Protocol}

Differentiation of ESC into the neural lineage was carried out in hanging drop culture, based on methods described by Okabe et al. [52] and Bibel et al. [103] In brief, stem cell suspensions $\left(3.75 \times 10^{4}\right.$ cells $\left./ \mathrm{ml}\right)$ were placed on ice before the initiation of the culture. Drops $(20 \mu \mathrm{l})$ containing 750 cells in CM were placed onto the inner side of the lid of a $90 \mathrm{~cm}$ Petri dish filled with phosphate-buff- 
ered saline (PBS) (Gibco BRL, Gaithersburg, MD) and incubated at $37{ }^{\circ} \mathrm{C}$, $90 \%$ relative humidity and $5 \% \mathrm{CO}_{2}$. After 3 days of hanging drop culture embryoid bodies (EB) had formed and were subsequently transferred to bacterial Petri dishes (Greiner Bio-one, Frickenhausen, Germany) containing CM supplemented with $0.5 \mu \mathrm{M}$ retinoic acid (RA) (Sigma-Aldrich, Zwijndrecht, The Netherlands). On day 5, EB were plated on laminin (Roche, Basel, Switzerland) coated dishes (Corning Incorporated, Corning, NY, USA) in LS medium supplemented with $2.5 \mu \mathrm{g} / \mathrm{ml}$ fibronectin (Invitrogen, Carlsbad, CA USA). One day later, on day 6 , the LS medium was replaced by ITS medium. In the short protocol, on day 7, EB were washed with phosphate buffered saline (PBS) (Gibco BRL, Gaithersburg, MD, USA) and incubated in cell dissociation buffer (Gibco BRL, Gaithersburg, MD, USA) for 3 minutes. Then EB were carefully detached from the Petri dish and replated on poly-L-ornithine (SigmaAldrich, Zwijndrecht, The Netherlands) and laminin coated dishes in N2 medium. After 6 hours incubation, basic fibroblast growth factor (bFGF) (Strathmann-Biotec AG, Englewood, CO, USA) was added to the medium at a concentration of $10 \mathrm{ng} / \mathrm{ml}$. Concerning the medium and long protocols, ITS medium was replaced every other day until replating of the EB was performed on day 10 or day 13 for the medium or long protocol, respectively. After replating the $\mathrm{EB}$, the $\mathrm{N} 2$ medium supplemented with bFGF was replaced every other day for 7 days in all three protocols (Figure 1).

\section{Antibodies}

The following primary antibodies were used: rabbit anti BIII-tubulin (1:2000) (Sigma-Aldrich, Zwijndrecht, The Netherlands), mouse anti nestin (1:50 (Millipore, Billerica, MA, USA), rabbit anti Glial Fibrilary Acidic Protein (GFAP) (Dako, Glostrup, Denmark), mouse anti Stage specific protein antigen-1 (1:400) (SSEA1) (1:400) (Millipore, Billerica, MA, USA), myelinassociated oligodendrocyte basic protein (1:50) (MOBP) (Santa Cruz Biotechnology Inc, Santa Cruz, CA, USA), Oligodendrocyte marker O4 (1:50) (Sigma-Aldrich, Zwijndrecht, The Netherlands). Secondary antibodies were polyclonal swine anti rabbit FITC (Dako, Glostrup, Denmark), rat anti mouse IgG1-PE (BD Biosciences, Franklin Lakes, NJ, USA), rat anti mouse IgG1-FITC (BD Biosciences, Franklin Lakes, NJ, USA), APC anti mouse IgM (Biolegend, San Diego, CA, USA), donkey anti rabbit IgG Cy3 (Jackson ImmunoResearch Europe Ltd, Suffolk, UK). In addition, TO-PRO-3 (1:1500) (Invitrogen, Carlsbad, CA, USA) was used as nucleus staining.

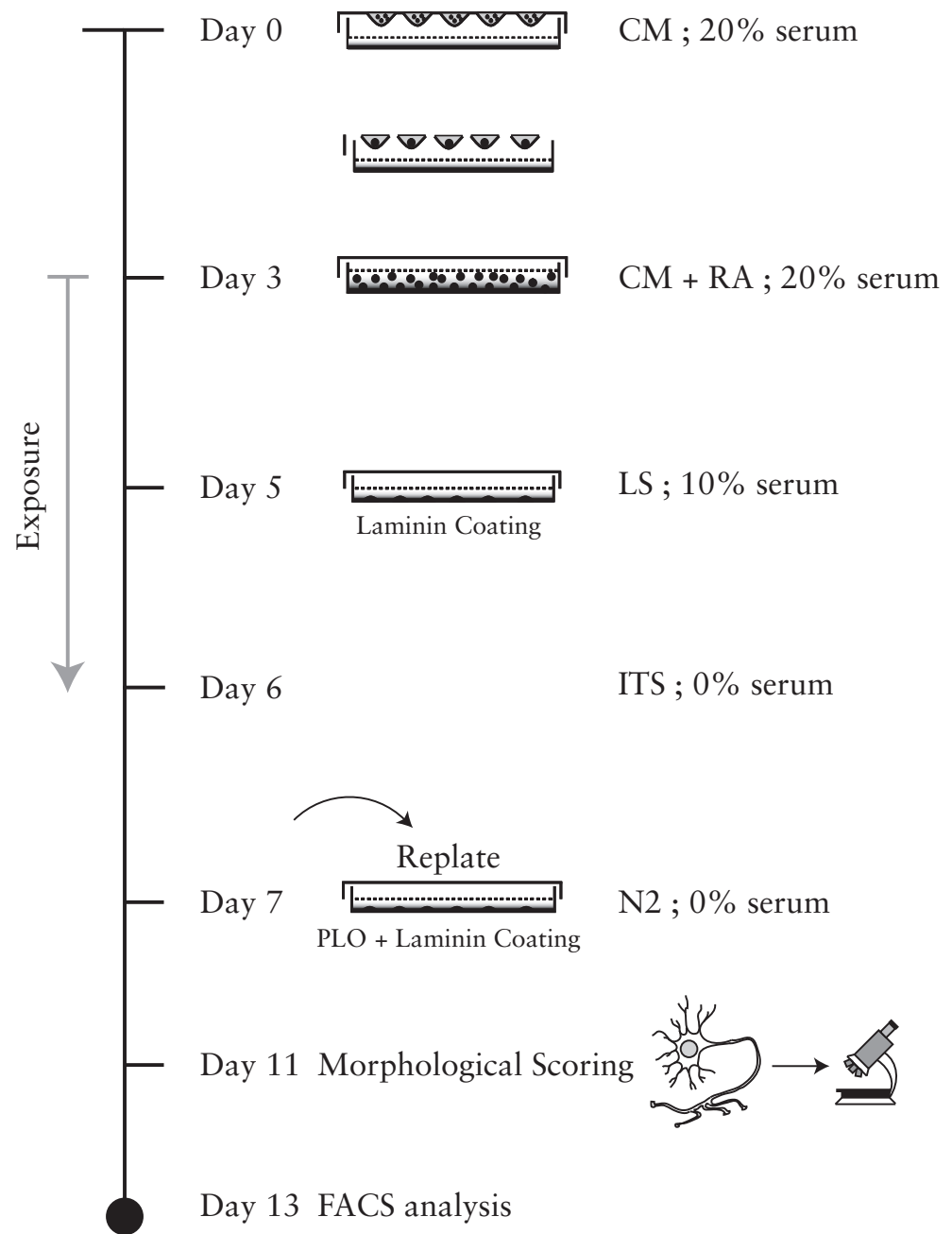

Figure 1 Neural differentiation short protocol. After a 3 day formation of embryoid bodies (EB) in hanging drops in $20 \%$ serum containing medium (CM), EB were cultured in bacteriological dishes in CM with retinoic acid (RA) for 2 days. At day 5 EB were plated on laminin coated dishes in $10 \%$ serum containing medium (LS). At day 6 the medium was refreshed with serum free medium (ITS). At day 7 EB were replated on poly-L-ornithin/laminin coated dishes and serum free 72 medium ple 11 and flow cypplemented with bFG. Morphological scoring was performed on day 11 and flow medium and long protocol, the ITS phase had a duration of 4 and 7 days, respectively. 


\section{Immunocytochemistry}

Cells were washed with ice-cold PBS, fixed with cold $2 \%$ paraformaldehyde (Sigma-Aldrich, Zwijndrecht, The Netherlands) in PBS for 5 minutes and washed once with PBS. Then the cells were incubated for 5 minutes in HEPES buffered saline solution (HBSS) (Lonza, Walkersville, MD, USA) supplemented with $0.1 \%$ saponin (Sigma-Aldrich, Zwijndrecht, The Netherlands) and incubated for 10 minutes in glycine (Sigma-Aldrich, Zwijndrecht, The Netherlands) in PBS. Cells were washed three times 5 minutes in PBS with 1\% albumin from bovine serum (BSA) (Sigma-Aldrich, Zwijndrecht, The Netherlands) and $0.1 \%$ saponin after which the sample was exposed to the primary antibody diluted in HBSS with $0.1 \%$ saponin for 30 minutes at room temperature (RT). Then the cells were washed three times 5 minutes in PBS with $1 \%$ BSA and $0.1 \%$ saponin, after which the cells were exposed to the secondary antibody diluted in $1 \%$ BSA and $0.1 \%$ saponin for 30 minutes at RT. The cells were washed two times 5 minutes with HBSS with $0.1 \%$ saponin in PBS and were treated with TO-PRO3 in PBS for 15 minutes at RT in the dark and afterwards washed once with PBS. Cells were closed in Vectashield (Vector Laboratories Inc, Burlingame, CA, USA) and the glass slide was closed using nailpolish. Samples were examined under a BX51 fluorescence microscope (Olympus, Zoeterwoude, The Netherlands) with CellF software for analysis (Olympus, Zoeterwoude, The Netherlands). Observations were performed under the fluorescence microscope. With negative ( - ) scoring, there were no positive cells present. With $(++)$ scoring, the highest number of cells positive for the marker was observed in the cultures. With (+) scoring, only smaller groups of the cell type were present. At least 3 samples per experiment were examined. For each differentiation protocol studied, at leas 3 experiments were performed.

\section{Flow Cytometry}

Cell types present at the end stage of the three protocols with varying culture times were counted by using fluorescent activated cell sorting (FACS). EB and cells in culture were dissociated for 30 minutes using cell dissociation buffer and resuspended into a single cell suspension. Cells were then washed with PBS and permeabilised with $4 \%$ paraformaldehyde for 30 minutes at RT. Cells were subsequently washed with FACS buffer $(0.5 \%$ BSA, $5 \mathrm{mM}$ ethylenediaminetetraacetic acid (EDTA) (Sigma-Aldrich, Zwijndrecht, The Netherlands) in PBS), permeabilised with permeabilization buffer $(0.15 \%$ saponin in PBS) for 30 minutes at RT and again washed three times with wash buffer (1\% BSA, $0.15 \%$ saponin in PBS) and incubated with the primary antibodies (anti- $\beta$ III-tubulin, anti-nestin and anti-SSEA1) diluted in wash buffer for 30 minutes at RT (see antibody section). After a triple wash with wash buffer, cells were incubated with the secondary fluorescent labeled (FITC, PE and APC) antibodies diluted in wash buffer for 30 minutes at RT in the dark. Cells were washed three times with wash buffer and resuspended in FACS buffer. Cells were analyzed using a FACSCalibur flowcytometer (BD Biosciences, Franklin Lakes, NJ, USA) and CellQuest Pro software (BD Biosciences, Franklin Lakes, NJ, USA)

\section{RNA isolation and Real Time Polymerase Chain Reaction (RT-PCR)}

Total RNA was isolated from differentiated ESC at days 5, 6 and 7 and at the end stage of the short (day 13), medium (day 17) and long (day 20) protocols using the Qiagen RNEasy Mini Kit (Qiagen Benelux, Venlo, The Netherlands) following manufacturer's instructions. RNA concentration and integrity was determined using the Nanodrop (Isogen Lifescience, de Meern, The Netherlands) and the 2100 Bioanalyzer (Agilent, Santa Clara, CA, USA), respectively. For conversion to cDNA 50 ng total RNA was reversed transcribed using reverse transcriptase, primers and probes, obtained from Applied Biosystems (High capacity cDNA reverse transcriptase kit) (Applied Biosystems Inc, Foster City, CA, USA). RT-PCR was performed using Taqman ${ }^{\circledR}$ fas universal PCR (Applied Biosystems Inc, Foster City, CA, USA) and specific TaqMan® Gene Expression Assays (Applied Biosystems Inc, Foster City, CA, USA) for each gene studied: Ectodermal genes studied were Sox1, an early marker for neural cell fate [112], paired box gene 6 (Pax6) a marker for early neural differentiation and radial glia cells [113] and nestin a neural precursor marker [114] [115]. Endodermal genes studied were Gata4, a transcription factor which activates endoderm specific gene products [116], alpha fetal protein $(A F P)$, a serum protein secreted by visceral endoderm [117, 118] and hepatocyte nuclear factor 4 alpha $(H N F 4 \alpha)$, a transcription factor which regulates expression of several hepatic genes [119]. Mesodermal genes studied were $T$, involved in formation of posterior mesoderm, an early peaking mesodermal marker [120], Nkx2-5, an early mesodermal marker [121] and myosin heavy chain 6 (Myb6), a marker for myosin [122].

For mature neural cells the following markers were used: markers for glutamatergic neurons, vesicular glutamate transporter 1 and 2 (VGluT1 and VGluT2) [123], for GABA-ergic neurons, vesicular GABA transporter (VGAT) 124] and glutamate decarboxylase 65 (GAD65) [125], tyrosine hydroxylase $(\mathrm{TH})$ for dopaminergic neurons [126], Brn3 for sensory neurons [127], HB9 for motor neurons [128] and GFAP as a marker for astrocytes [129].

RT-PCR was performed using the 7500 Fast Real-Time PCR System (Applied Biosystems Inc, Foster City, CA, USA). All gene expression assays were 
validated in the test system using the HPRT and GUSb housekeeping genes and gene expression for all genes in the experiments was corrected for these stable housekeeping genes.

\section{Cytotoxicity Assay}

To determine cytotoxicity of methylmercury chloride (MeHg) (Sigma-Adrich, Zwijndrecht, The Netherlands), ESC were plated in 96-well plates at 500 cells per well in CM supplemented with LIF and allowed to attach for 2 hours before $\mathrm{MeHg}$ exposure at concentrations of $3.3 \mathrm{nM}, 33 \mathrm{nM}, 100 \mathrm{nM}, 200$ $\mathrm{nM}, 550 \mathrm{nM}$ and $1 \mu \mathrm{M}$. Cells were subsequently cultured for 5 days at $37^{\circ} \mathrm{C}$ and $5 \% \mathrm{CO}_{2}$, with a medium renewal containing $\mathrm{MeHg}$ for exposure on day 3. On day 5 (approximately $80 \%$ cell confluence in non-exposed controls), CellTiter-blue (Promega, Leiden, The Netherlands) was added to each well and incubated for 2 hours. After the incubation period, fluorescence was read using a FLUOstar spectrofluorometer (FLUOstar Optima, BMG Labtech, de Meeren, The Netherlands) at 544 (excitation) and $590 \mathrm{~nm}$ (emission). Three independent experiments were performed. Concentration-response analysis was performed using PROAST (Possible Risk Obtained from Animal Studies) software (RIVM, Bilthoven, The Netherlands).

\section{Morphological scoring and flow cytometry for effects on}

\section{neural outgrowth}

The short 13 day neural differentiation protocol was used to test the effects of $\mathrm{MeHg}$ on neural differentiation (Figure 1). $\mathrm{MeHg}$ was tested during different phases of the protocol: the serum-containing phase (day 3-6), the serum-free phase (day 6-13), or the combined exposure protocol (day 3-13). MeHg was diluted in DMSO and added to the medium to final concentrations in culture of $2.5 \mathrm{nM}, 25 \mathrm{nM}$ and $250 \mathrm{nM}$ and control EB were treated with $0.01 \%$ DMSO. Effects were determined using morphological assessment on the extent of neural outgrowth at day 11 using an IX51 inverted microscope (Olympus, Zoeterwoude, The Netherlands) with CellD software (Olympus, Zoeterwoude, The Netherlands). Morphological neural outgrowth was scored as $<75 \%$ or $\geq 75 \%$ of the neural corona surrounding each $\mathrm{EB}$, irrespective of the distance of outgrowth from the EB. Flow cytometric analysis was performed on day 13 to study (neural)-differentiation key proteins ( $\beta$ III-tubulin, nestin and SSEA1) Morphological scoring (day 11) and flow cytometric analysis (day 13) were performed on different days for logistic reasons. Three individual experiments (each with $\mathrm{n}=3$ ) were performed.

\section{อి}

\section{Results}

Abbreviation of the neural differentiation protocol

In order to enhance throughput of the neural differentiation assay, shorte versions of the original combined 20 days neural differentiation protocol (long protocol) were designed and tested. Initial morphological observations showed that neural differentiation was present from day 7 onwards. Therefore the ITS phase was abbreviated to study its effect on the course of neural differentiation. With this in mind, we developed two new protocols, in which the ITS phase was reduced from the original 7 days to 4 (medium protocol) and 1 (short protocol) days, respectively. These three protocols (long, medium and short) were characterized over time both morphologically as well as using immunocytochemistry, RT-PCR and flow cytometric analysis.

\section{Morphology}

Comparing across all three protocols using light microscopy, we identified similar differentiation patterns morphologically, including similarities in appearance and size of neurite outgrowth (Figure 2).

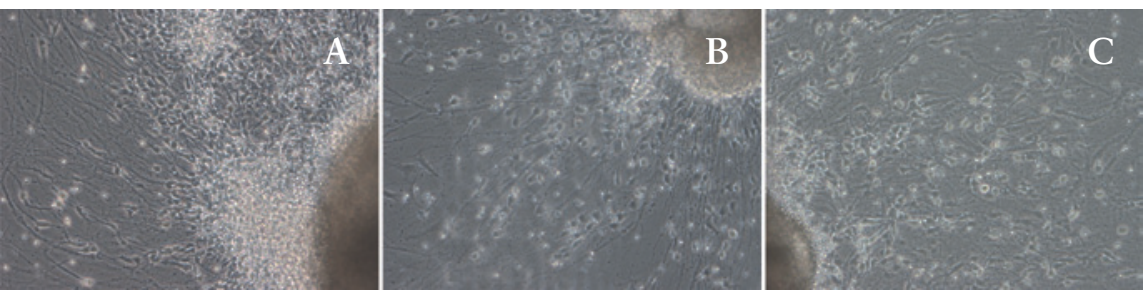

Figure 2 Typical final morphological appearance of the A) short (Day 13) (10x), B) medium (Day 17) (10x) and C) long (Day 20) protocols (10x)

\section{Immunocytochemistry}

Fluorescent staining of key proteins for neural differentiation was performed using immunocytochemistry and studied under a fluorescence microscope (Table 1 and Figure 3). Expression of SSEA1 was found abundantly from the start of the protocol at day 0 to day 6 and was reduced over time to low expression levels. Expression of nestin positive cells was observed from day 5 onwards through the whole EB and decreased after replating of the EB over time and was still present at the end stages of all three models. The first $\beta I I I-t u b u l i n$ positive cells were observed from day 5 onwards and the number 
of $\beta$ III-tubulin positive cells increased over time in all three models. The first signs of GFAP positive cells were observed in the EB at day 13 in all three models. From day 13 onwards, GFAP positive cells migrated out of the EB, resulting in GFAP positive cells mainly found in the EB in the short protocol, and GFAP positive cells both in- and outside the EB at the end of the medium and long protocols. During the whole culture period, no positive staining for oligodendrocytes was observed using the markers $\mathrm{O} 4$ and MOBP.

Table 1 Presence of neural differentiation proteins over time observed by fluorescence microscopy during the shared first phase and at the end phase of each of the three protocols

\begin{tabular}{|c|c|c|c|c|c|c|c|c|c|}
\hline Antibody & Function & $\begin{array}{l}0 \\
\vec{D} \\
\stackrel{\Xi}{a}\end{array}$ & $\stackrel{n}{\text { D. }}$ & $\stackrel{+}{\vec{\sigma}}$ & 站 & 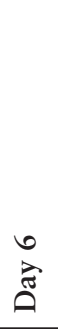 & 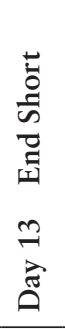 & 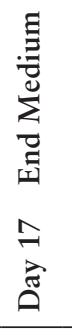 & 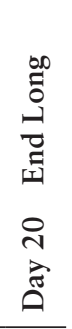 \\
\hline SSEA1 & Pluripotency & ++ & ++ & ++ & ++ & ++ & + & + & + \\
\hline Nestin & Neural Precursor & - & - & - & + & ++ & ++ & ++ & ++ \\
\hline$\beta I I I-T u b u l i n$ & Neurofilament & - & - & - & + & + & ++ & ++ & ++ \\
\hline GFAP & Astrocytes & - & - & - & - & - & + & ++ & ++ \\
\hline $\mathrm{O} 4$ & Immature Oligodendrocytes & - & nt & $\mathrm{nt}$ & $\mathrm{nt}$ & - & - & - & - \\
\hline MOBP & Mature Oligodendrocytes & - & $\mathrm{nt}$ & $\mathrm{nt}$ & $\mathrm{nt}$ & - & - & - & - \\
\hline
\end{tabular}

++ = abundantly present; + = low presence; - = not present; nt = not tested

\section{Flow Cytometry}

For all three protein differentiation markers ( $\beta$ III-tubulin, nestin and SSEA1) we did not observe a significant $(\mathrm{p}<0.05)$ difference between separate experiments within any of the three protocols (Figure 4A-C). Quantities of total protein a the end of culture were highly consistent and reproducible within each of the protocols (data not shown).

We observed a tendency of increasing numbers of positive cells with protocol duration for each differentiation marker studied (Figure 4D). This was reflected in significant difference of the percentage of $\beta$ III-tubulin positive cells when

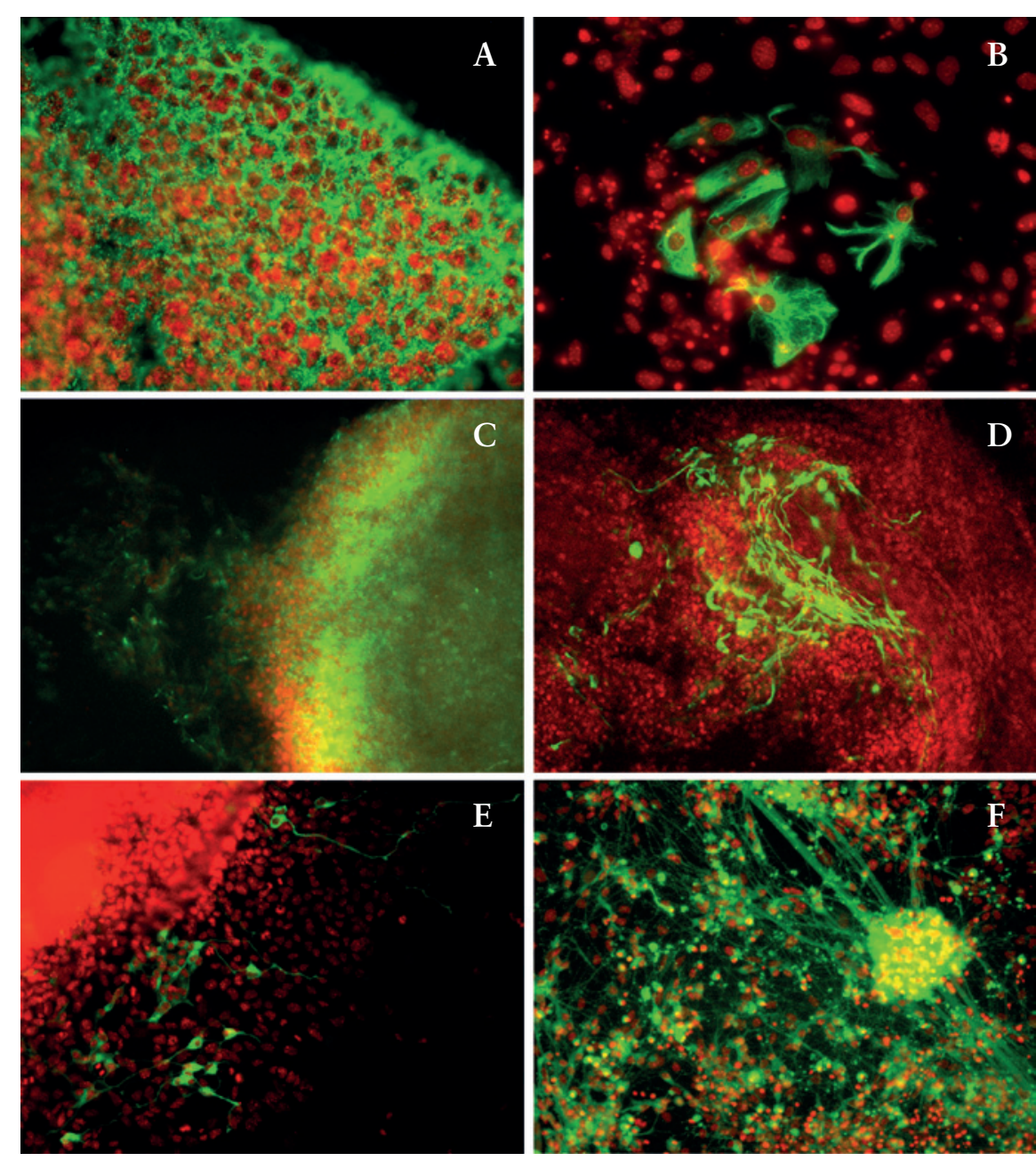

Figure 3 Fluorescence labeling of fixed cells. Protein staining in green, nucleus staining in red. A) SSEA1 staining at day 3 on frozen section of EB (20x); B) Astrocyte staining at day 13 of short protocol (20x); C) nestin staining at day $6(10 \mathrm{x})$ and D) at day 13 of the short protocol (10x); E) BIII-tubulin staining on day $6(10 \mathrm{x})$ and F) on day 13 of the short protocol $(10 \mathrm{x})$.

comparing the short and long protocol $(\mathrm{p}<0.01)$. No significant differences $(\mathrm{p}<0.05)$ were identified for the SSEA1 and nestin protein when comparing across all three protocols. 


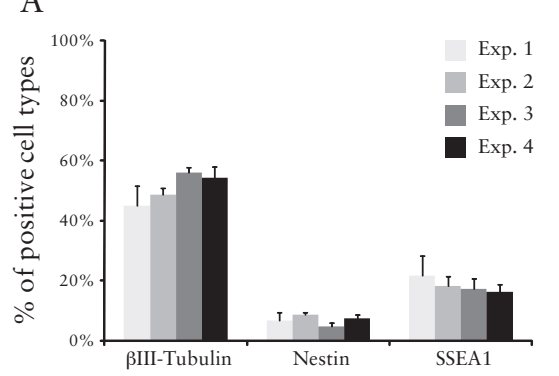

C

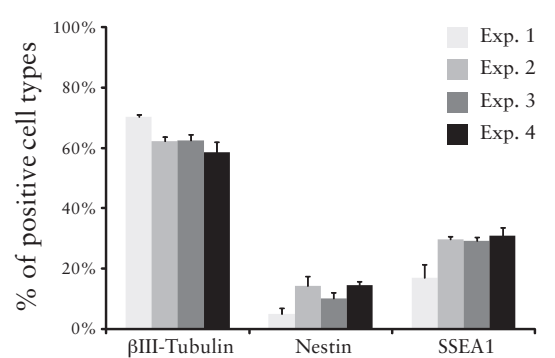

B

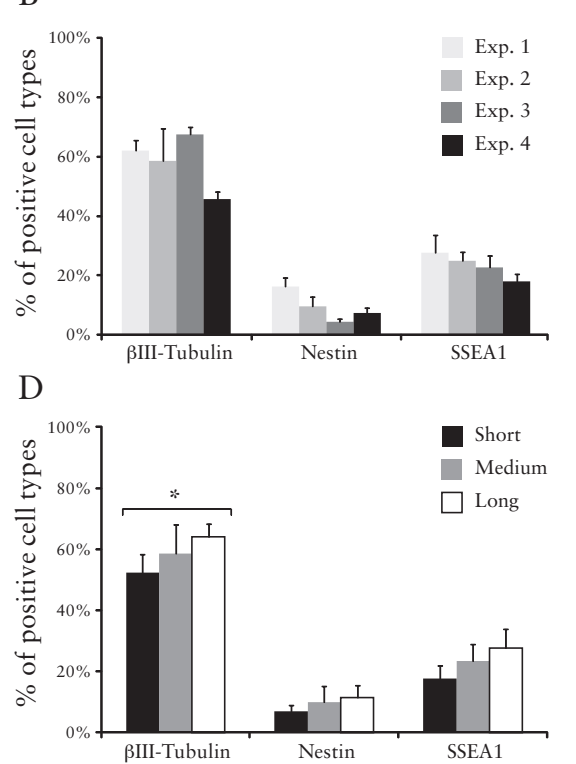

Figure 4 Reproducibility of the A) short, B) medium and C) long protocols by flow cytometric analysis for SSEA1, nestin and $\beta I I I-t u b u l i n(n=4$ per experiment, error bars in S.E.M.). D) Comparison of percentage of SSEA1, nestin and $\beta$ III-tubulin positive cells for the short, medium and long protocols by flow cytometric analysis. Each experiment was performed 4 times with $\mathrm{n}=4$ per experiment (error bars in S.E.M.)

\section{Early neural differentiation upon serum deprivation}

To further characterize the protocol, a series of differentiation markers was studied from day 5 to day 7 using RT-PCR during serum deprivation. This stage of the assay is shared by the three protocols studied. Two independent experiments were performed (Figure 5). Concerning the ectodermal markers, whilst Sox 1 and Pax6 did not show significant changes over time. The neural precursor marker nestin increased with time $(\mathrm{p}=0.009)$. No significant changes were observed for the early mesoderm marker $T$ and $N k \times 2-5$ and the cardiac muscle marker Myb6. Also no significant differences $(\mathrm{p}<0.05)$ were observed for the endodermal markers Gata4, AFP, and HNF4 $\alpha$. The neuron-type specific genes studied (VGluT2 p=0.007, GAD65 p=0.02, VGAT p=0.02) showed a particularly remarkable significant increased expression from day 5 to 7 in the protocol. The two independent experiments essentially showed the same trends over time. Thus, between day 5 and 7 of the protocol the cultures were shown to contain cells of each of the three germ layers, as well as various neural cell types with neural markers increasing with culture time.

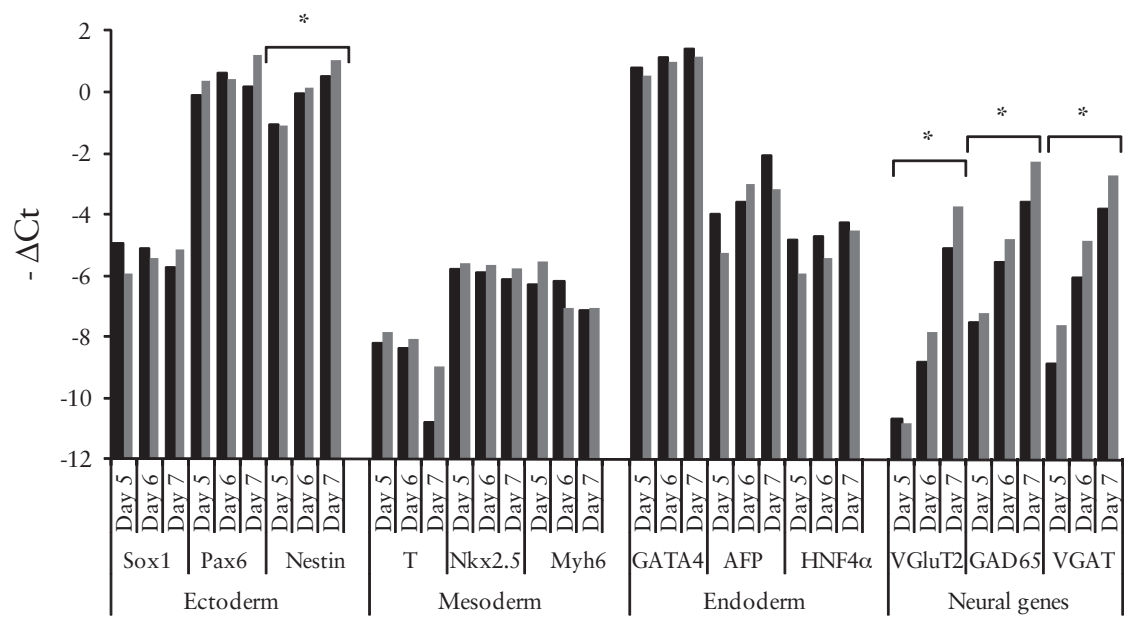

Figure 5 Gene expression for specific germ layers and neuron type genes. RT-PCR of ectodermal (Sox1, Pax6 and nestin), mesodermal (T, Nkx2-5 and Myb6), endodermal genes (Gata4, AFP and HNF4 $\alpha$ ) and specific neuron types (VGluT2, GAD65 and $V G A T)$ at day 5, 6 and 7 of the protocols was performed in two separate experiments. The following markers are significantly different over time according to one way Anova; nestin $\mathrm{p}=0.009 ;$ VGluT2 $\mathrm{p}=0.007 ;$ GAD $65 \mathrm{p}=0.02 ;$ VGAT $\mathrm{p}=0.02 .-\Delta \mathrm{CT}$ is the relative difference of the cycle of threshold $(\mathrm{Ct})$ of an investigated gene corrected for a housekeeping gene.

\section{Neural differentiation at endpoint of three models}

RT-PCR assessment of nine different neural markers at the end stage of each of the three protocols generally revealed no significantly different expression levels between protocols $(\mathrm{p}<0.05)$. The only exception was GFAP expression, which was significantly ( $\mathrm{p}=0.04$ short versus medium; $\mathrm{p}=0.003$ short vs. long) more intense in the medium and long protocol as compared to the short protocol (Figure 6). Nevertheless, the short protocol still showed significant levels of this marker. 


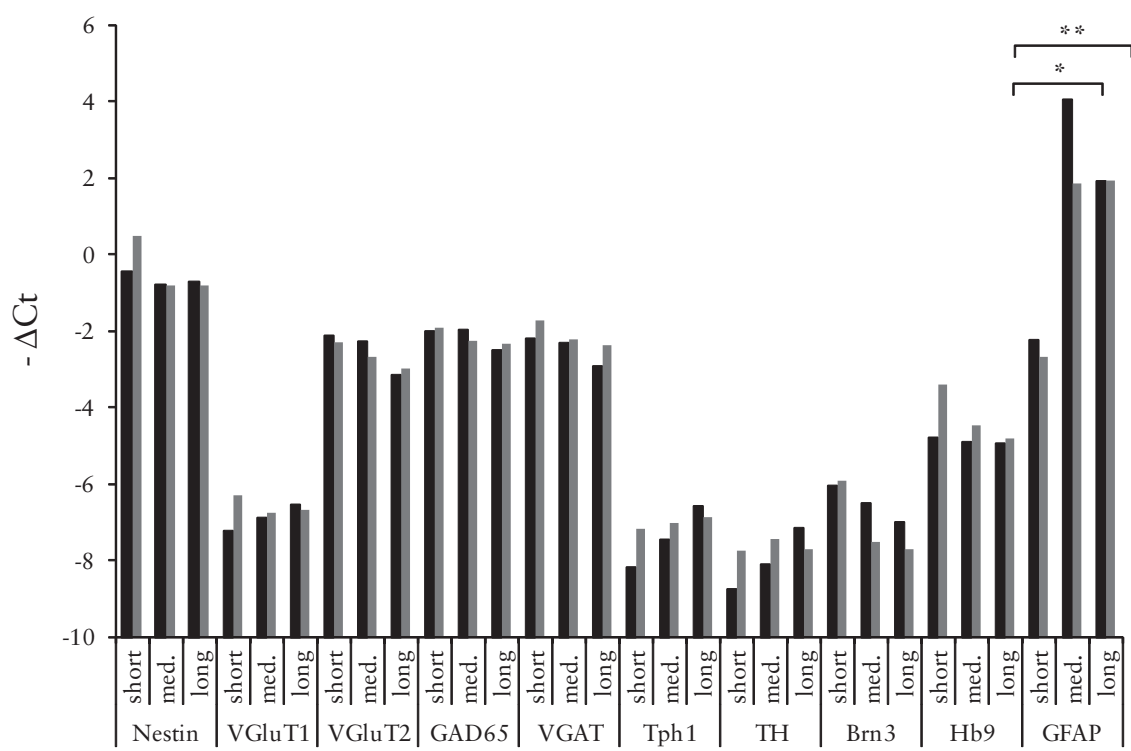

Figure 6 RT-PCR on neural precursors (nestin), specific neuron types (VGluT1, VGluT2, GAD65, VGAT, TH, Brn3 and Hb9) and astrocytes (GFAP) at the end stages of the short (day 13), medium (day 17) and long (day 20) protocols. " $\mathrm{p}=0.04$; $* * \mathrm{p}=0.003$

\section{Effects of the neurotoxicant $\mathrm{MeHg}$}

$\mathrm{MeHg}$ was used as a model compound to test effects in the neural differentiation protocol. In undifferentiated embryonic stem cells, using the Alamar Blue cytotoxicity assay, the half maximal inhibitory concentration for cytotoxicity (IC50) was $222 \mathrm{nM}$ (188.4 nM - 264.4 nM 95\% confidence interval) (Figure 7) (test performed in triplicate). This data was used as a reference level for $\mathrm{MeHg}$ IC50 in following experiments.

\section{Developmental toxicity: Morphological scoring}

Effects of $\mathrm{MeHg}(2.5,25$ and $250 \mathrm{nM})$ on cell morphology in the assay was tested after exposure during different phases of the short term protocol: the serum-containing phase (day 3-6), the serum-free phase (day 6-13), or these time windows combined (day 3-13) (Figure 8). Three independent experiments were performed in triplicate. EB outgrowth was morphologically scored at day 11. All three treatment periods showed a significant concentration-effect on neural outgrowth. At a concentration of $250 \mathrm{nM}$, an absence of neural

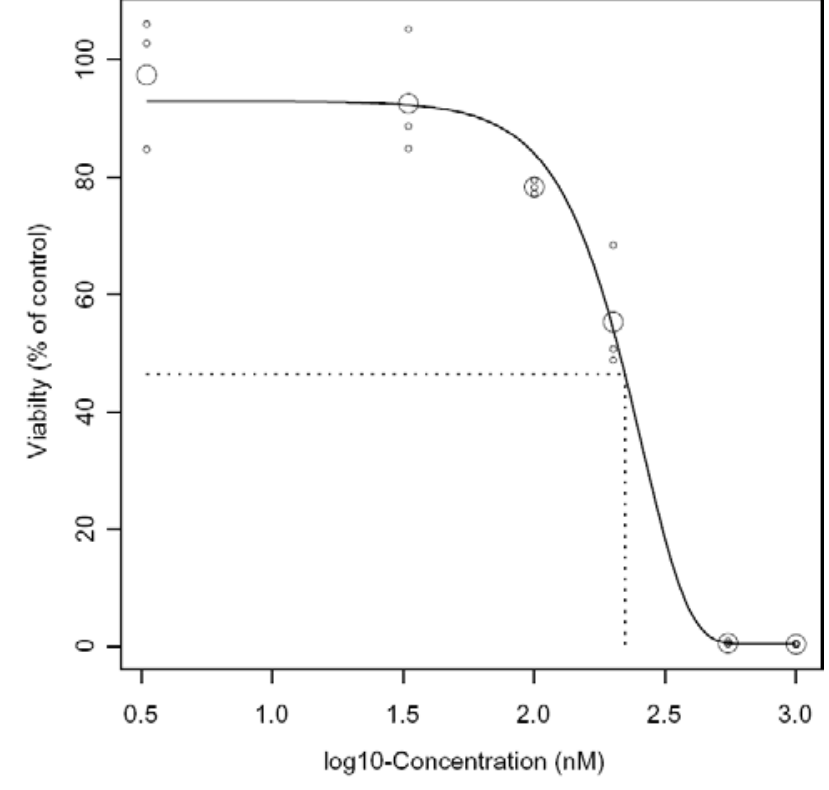

Figure 7 Alamar Blue cytotoxicity concentration-response of MeHg. IC50: $222 \mathrm{nM}$ (188 nM - $264 \mathrm{nM})$.

outgrowth in addition to cell death was observed after exposure during the day 6-13 and the day 3-13 protocol. This in contrast to the observed outgrowth after day 3-6 exposure, which indicates a higher sensitivity to $\mathrm{MeHg}$ in serum free medium compared to medium with serum. In addition, exposure to $\mathrm{MeHg}$ during the serum free only period showed a stronger effect at 2.5 and $25 \mathrm{nM}$ when compared to treatment during the combined exposure protocol.

\section{Developmental toxicity: Flow cytometric analysis}

Effects of MeHg on the percentages of cells positive for the markers SSEA1, nestin and $\beta$ III-tubulin were assessed after exposure during different phases of the protocol: the serum-containing phase (day 3-6) (Figure 9A), the serum-free phase (day 6-13) (Figure 9B), or the combined exposure protocol (day 3-13 (Figure 9C). Samples were analyzed using flow cytometry at the end stage of the short protocol on day 13. No significant differences were observed for $\beta I I I-t u b u l i n$ for all exposure windows and all concentrations tested $(\mathrm{p}<0.05)$. A significant increase of nestin positive cells $(\mathrm{p}<0.02)$ was observed at $250 \mathrm{nM}$ in all exposure protocols when compared to control. Also, a significant increase $(\mathrm{p}<0.002)$ of SSEA1 positive cells was observed at $250 \mathrm{nM}$ after exposure from day 6-13. 


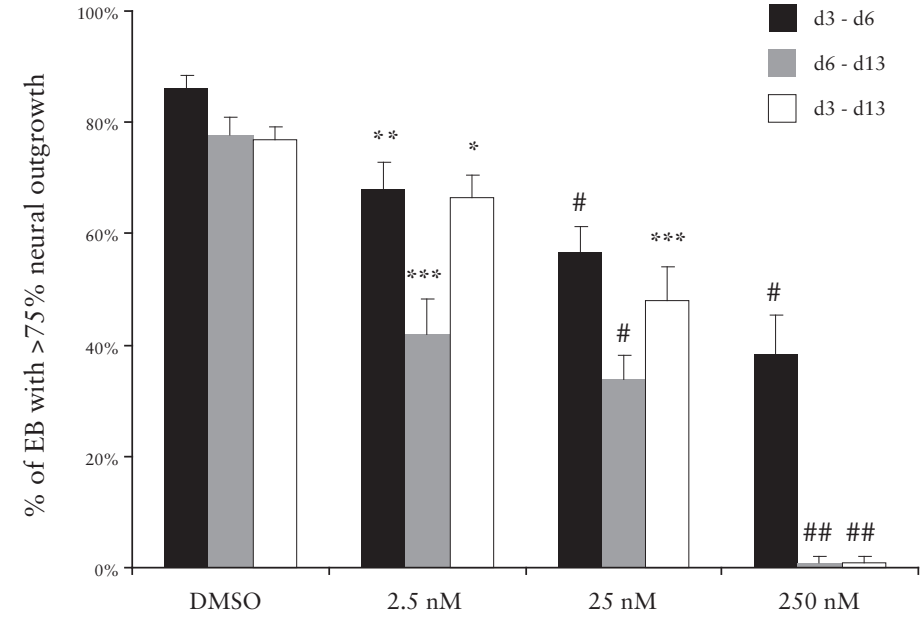

Figure 8 Morphological scoring. Percentage of EB with $>75 \%$ neural outgrowth after $\mathrm{MeHg}$ treatment $(2.5,25$ and $250 \mathrm{nM})$ during the serum containing phase of the protocol (day 3-5), the serum free phase of the protocol (day 6-13) or the combined exposure protocol (d3-13). * $\mathrm{p}<0.05, * * \mathrm{p}<0.005, * * * \mathrm{p}<0.005$, \# $\mathrm{p}<0.00005$, \#\# $\mathrm{p}<10^{-13}$. All differences are compared to DMSO control (Error bars in S.E.M.).

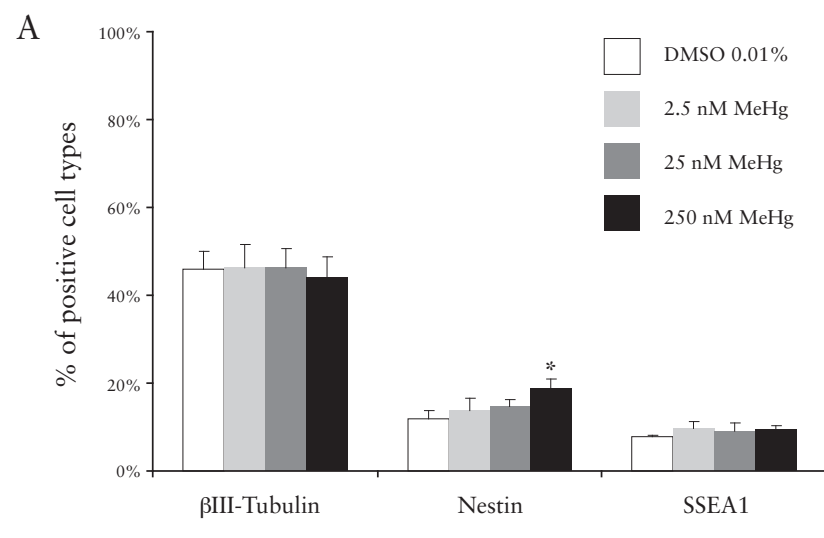

Figure 9 Flow cytometric analysis of the effect of $\mathrm{MeHg}$ on the percentage cells positive for the markers SSEA1, nestin and BIII-tubulin after treatment with 2.5, 25 or $250 \mathrm{nM} \mathrm{MeHg}$ during A) day 3-5, B) day 6-13 or C) day 3-13. " $\mathrm{p}<0.02$, ** $\mathrm{p}<0.002$, all differences in comparison to vehicle control (Error bars in S.E.M.).
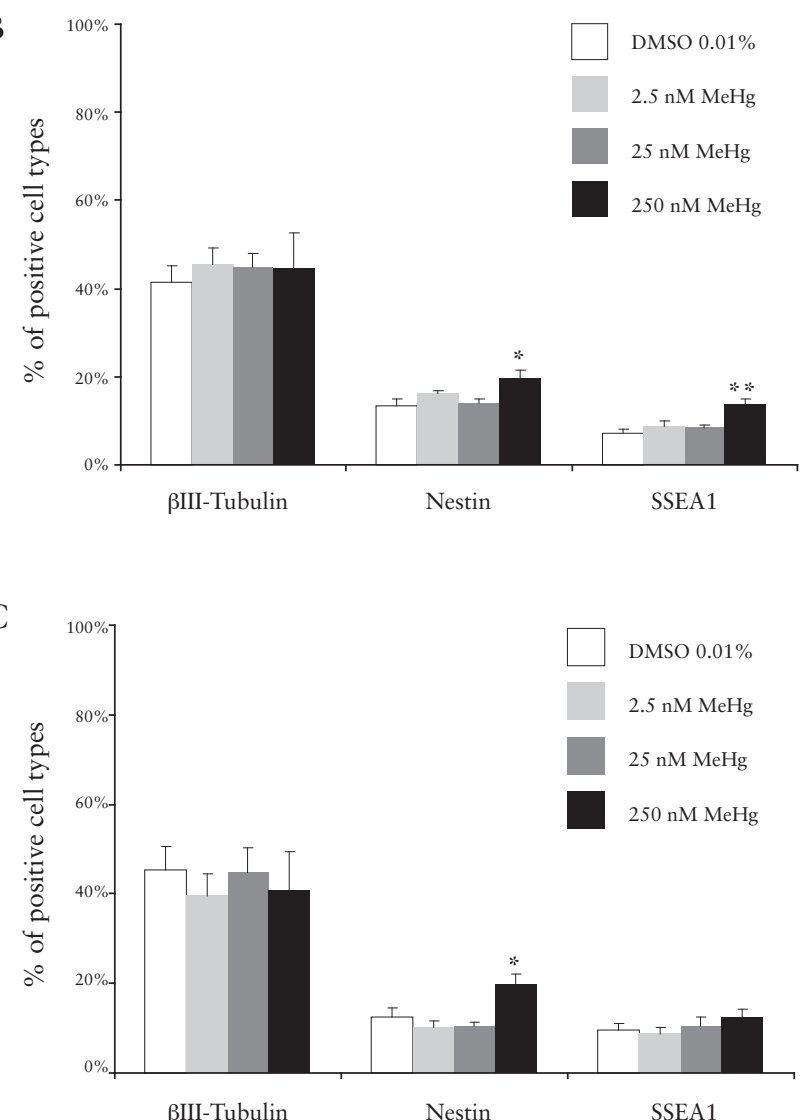

Figure 9 Continued. ${ }^{*} \mathrm{p}<0.02,{ }^{* *} \mathrm{p}<0.002$, all differences in comparison to vehicle control (Error bars in S.E.M.). 


\section{Discussion}

\section{Abbreviation of the protocol}

The first aim of this study was to design an efficient ESC neural differentiation assay. To abbreviate the published 20 day protocols, the ITS phase was reduced from 7 days in the long protocol to 4 days and 1 day in the medium and shor protocols, respectively. Morphological observations (Figure 2) and immunocytochemical staining of key markers for pluripotency, neural precursor cells and mature neural cells (Figure 3) showed similar profiles when comparing al three protocols. Flow cytometry using these markers showed that each of the protocols gave reproducible results, indicating the robustness of the mode (Figure 4). Expression levels of neural precursor genes and genes for a range of specific neuron types (Figure 6) showed a very similar expression profile at the end stage of all three protocols. These findings confirmed the similarity of neural differentiation in the three protocols and showed at the same time the feasibility of the abbreviated protocol.

Concerning glial cell differentiation, immunocytochemistry showed that the amount of GFAP positive cells at the end stage of the short protocol was visibly lower compared to the medium and long protocol. In the short protocol, GFAP positive cells were observed mainly inside or very near to the EB, whereas in the other protocols, GFAP positive cells were abundant both in and outside the EB (data not shown). Similarly a lower expression of the GFAP gene (Figure 6 also showed a lower expression in the short protocol compared to the other protocols, confirming the immunocytochemical observations. Although the amount of GFAP positive cells appeared lower in the short protocol, still a considerable number of cells expressing GFAP were observed, retaining the possibility that effects on differentiation towards astrocytes can be monitored Together, these data show that abbreviation of the ITS phase from 7 days to 1 day provides similar neural differentiation profiles. Therefore, the short protocol was used for further experiments.

\section{Advantages of the protocol for developmental toxicity testing}

Our abbreviated neural differentiation assay has a number of advantages ove previously described systems for early neurodevelopmental toxicity screening. An often used method for in vitro neurodevelopmental toxicity makes use of neural progenitor cells (NPC) [130-132]. These cells are already in an advanced stage of neural differentiation as compared to ESC. Therefore, early effects on the formation and balance between ectoderm, mesoderm and endoderm germ layers and their consequences for neural differentiation cannot be detected using NPC. In our ESC neural differentiation culture, RT-PCR showed that especially ectodermal and endodermal, but also mesodermal gene expression was present, which offers that the assay captures a broader spectrum of developmental mechanisms, suggesting the possibility for improved prediction for compounds indirectly affecting neural cell differentiation.

Our system uses standardized EB formation with the hanging drop method and maintains EB structure throughout the entire protocol, which support stable culture conditions. In some developmental toxicity studies, ESC monolayer culture was used for neural differentiation [106], or EB were dissociated to single cells during the protocol [41], creating a less stable protocol. In our model EB are replated, instead of dissociated, to keep the multi-cellular spatial structure of the EB intact. EB formation furthermor allowed differentiation to mimic the in vivo developmental process in a more complete manner compared to monolayer differentiation, due to the presence of all three germ layers [38].

\section{Characterization of the short protocol}

RT-PCR showed that early neural differentiation genes, such as Sox1 and Pax6, were stably expressed between days 5 and 7 (Figure 5). Nestin expression was increasing from days 5 to 7, indicating increasing neural differentiation, with a comparable expression level at day 13 of the culture compared to day 7 (Figure 6). Expression of several endodermal and ectodermal genes was detected from day 5-7. Mesodermal gene expression was also detected in our assay, although the genes studied were expressed at lower levels compared to the ectodermal and endodermal differentiation. These results showed that al three major embryonic differentiation routes were affected in the culture from day 5 through day 7 . Furthermore, we showed that in our model GABA-ergic and glutamatergic neurons are formed early on, at least from day 5 onwards. These neural cell types belong to the first classes of neurons to develop in vivo and have an important role in controlling cell division, neuronal migration and maturation during neural development [133-135]. It has been shown that the neurotransmitters of these two neuronal classes have an effect on furthe neural differentiation pathways in vivo and in vitro. At the end of the culture (day 13), a higher expression for VGluT2 is observed compared to low expression levels for VGluT1, which are both markers for different types of glutamatergic neurons. In vivo, it is shown that during embryonic development, VGluT2 expressing glutamatergic neurons are more abundant compared to VGluT1 expressing glutamatergic neurons [135]. After birth VGluT2 positive neurons decline in number and VGluT1 positive neurons increase in number [136]. The higher expression of VGluT2 expression compared to VGluT1 expression at the end stage of our culture reflects the in vivo situation during 
development. Taken together, the abbreviated protocol appeared to display an array of characteristics reminiscent of germ layer development and neuronal differentiation, which may be useful in the detection of chemicals affecting these processes.

\section{Neurodevelopmental toxicity testing}

Previous studies performed in our laboratory showed that proliferation and differentiation of ESC in the cardiac EST (ESTc) are highly intertwined processes [137]. It was recommended that, to largely limit exposure to the differentiation phase of the assay, it would be advantageous that EB be exposed to compounds from day 3 in the assay onwards [137]. Up to day 3, the ESTc and our neural differentiation assay protocols are identical. Therefore, in the present study $\mathrm{MeHg}$ exposure was performed from day 3 onward. EB were treated either during the serum-containing phase (day 3-6), the serum free phase (day 6-13), or the combined exposure protocol (day 3-13), in order to determine whether the presence of serum would influence the sensitivity of the cells. Morphological scoring for neural outgrowth was performed on day 11 (Figure 8) and flow cytometric analysis was performed on day 13 (Figure 9). For morphological scoring, all three treatments showed clear dose-responses in the $2.5-25 \mathrm{nM}$ range, indicating that the assay is highly sensitive to $\mathrm{MeHg}$. In an earlier study, effects of MeHg on MAP2 gene expression in human ESC were shown at $320 \mathrm{nM}$ [138] and a study using human NSC [132] reported an effect on neurite outgrowth at concentrations between 500 and $700 \mathrm{nM}$ MeHg. Furthermore, a study using mouse ES cell neural differentiation [41] using a variation of the long protocol based on the protocol described by Okabe et al.[52] found an effect on Mtap2 gene expression after 14 days of neural differentiation at a concentration of $100 \mathrm{nM} \mathrm{MeHg}$. In our assay, significant effect is observed at $2.5 \mathrm{nM} \mathrm{MeHg}$, indicating that our model appeared relatively sensitive in detecting effects of $\mathrm{MeHg}$ on neural differentiation.

The $\mathrm{MeHg}$ concentration-responses showed a more severe effect after day 6-13 exposure as compared to day 3-6 and day 3-13 exposure protocols (Figure 8). The fact that the latter two are similar indicates that early effects largely determine the overall outcome of the assay. The culture composition at the start of day 6-13 treatment in terms of cell populations is likely different with and without previous day 3-6 exposure, which may explain the more severe effect observed after day 6-13 treatment only. The complete absence of neura outgrowth observed exclusively after late (day 3-13 and day 6-13) exposures to the highest concentration tested may be related to the cytotoxicity of the compound observed at this concentration (Figure 7), which is likely more pronounced under serum free conditions. The suggestion that high exposure may modulate cell population ratios was supported by flow cytometric analysis performed for the pluripotency marker SSEA1, the neural precursor marker nestin and the neuron specific cytoskeletal protein BIII-tubulin (Figure 9). Significantly more nestin positive cells were identified in a concentration-dependent fashion in culture using all three exposure protocols. Also, after high concentration day 6-13 treatment, the number of SSEA1 positive cells was significantly higher compared to control. These findings suggest that $\mathrm{MeHg}$ exposure resulted in increased undifferentiated and neural precursor cells in the assay relative to $\beta$ III-tubulin positive neural cells. In addition, the observed SSEA1 increase implies that $\mathrm{MeHg}$ may be more toxic to neural cells than to undifferentiated cells.

The morphological effect of reduced neural outgrowth in our assay is not reflected in the percentage of $\beta$ III-tubulin positive cells after $\mathrm{MeHg}$ treatment. However, it has been shown that $\mathrm{MeHg}$ has an effect on dendrite and axon growth in PC12 cells [139] and in primary mouse dopaminergic mesencephalic cells [140]. In another study, the effect of $\mathrm{MeHg}$ on a range of neural differentiation related genes was studied on mouse ESC neural differentiation [41]. Only the marker Mtap2 showed a significant decrease at non-cytotoxic $\mathrm{MeHg}$ levels. In addition to its role in the maturation of neurons, Mtap2 is also associated with dendrite and axon growth [141]. Our combined morphologica and flow cytometric data suggest that $\mathrm{MeHg}$ did not affect the number of III-tubulin positive cells, but did reduce the growth and migration of neurites.

In summary, we have successfully designed an abbreviated 13-day mouse ESC neural differentiation protocol. We have shown that each of the three embryonic germ layers as well as multiple lineages of neural and other brain-associated cells were represented in our protocol. In addition, using a variety of differentiation markers we showed differential effects of $\mathrm{MeHg}$ exposure on differen cell types as well as on neuronal outgrowth. We consider these finding promising as we anticipate that the multi-lineage character of the assay may allow improved detection of neurodevelopmental toxicants in view of the large spectrum of possible target cell types present in the assay. The model is not yet ready to be used as a standardized assay for assessing developmental toxicity in vitro. The applicability of the assay for the detection of neurodevelopment toxicants awaits further assessment of assay performance with a series of neurodevelopment toxicants.

\section{Tox}




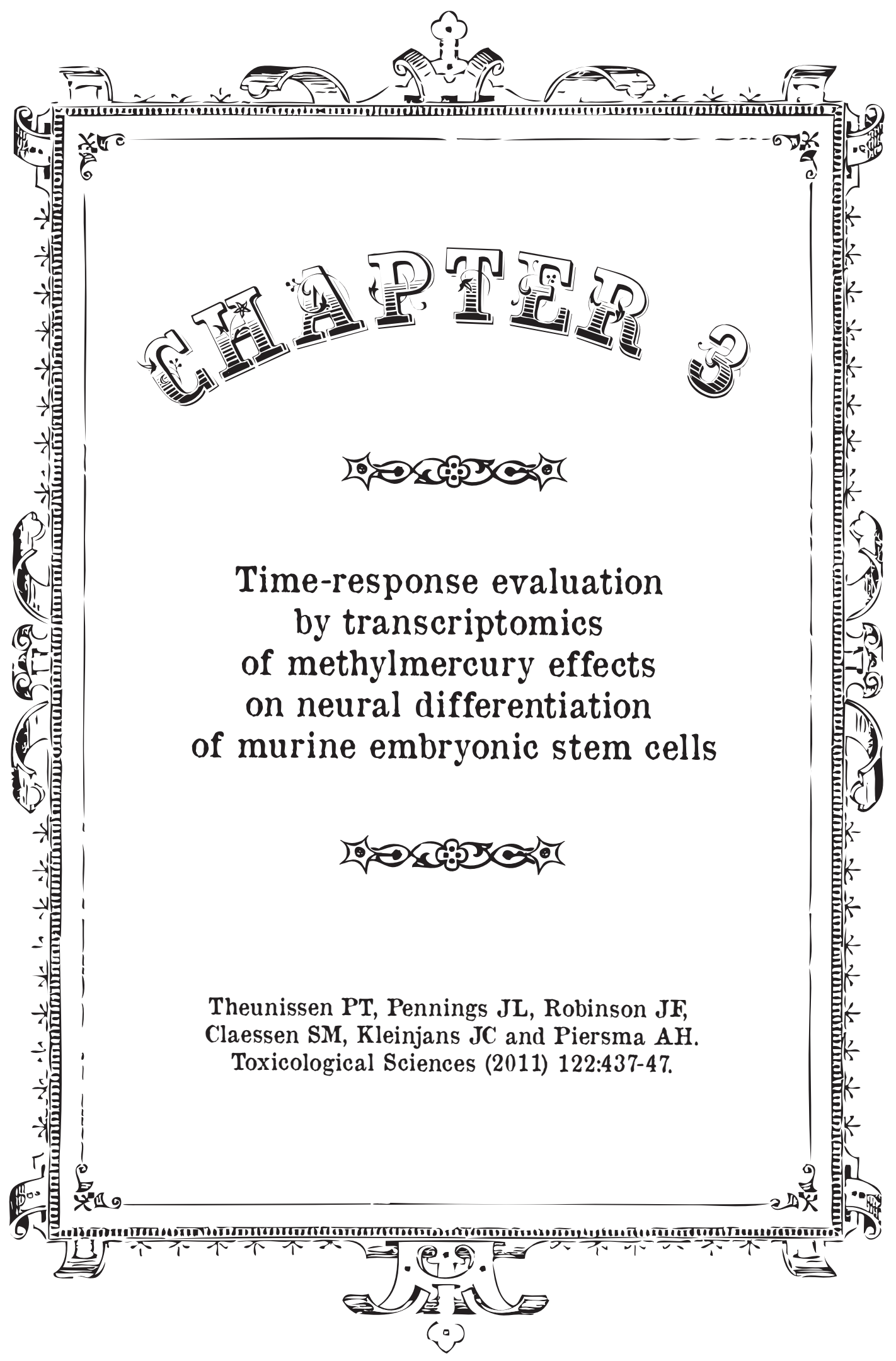




\section{Abstract}

URRENT globally harmonized OECD animal test guidelines for developmental toxicity require high numbers of experimental animals. To reduce animal use in this field, alternative developmental toxicity assays are highly desirable. We previously developed a dynamic in vitro model for screening effects of possible neurodevelopmental toxicants, using neural cell differentiation of pluripotent murine embryonic stem cells. To further mechanistically characterize the mouse neural embryonic stem cell test (ESTn) and to improve detection of possible neurodevelopmental toxicants, gene expression patterns were studied describing neural cell differentiation over time, as well as the impact on gene expression of exposure to the well known neurotoxicant methylmercury $(\mathrm{MeHg})$ A transcriptomics study was performed to examine whole-genome expression changes during the first seven days of the cell differentiation protocol. Specific gene clusters were identified and enrichment analysis of Gene Ontology (GO) terms and gene sets derived from literature was performed using DAVID and Tox-profiler. Over time, a decrease of blastocyst and trophectoderm GO-terms was observed, which included well characterized pluripotency genes. Furthermore, an increase of a range of neural development related GO-terms, such as neuron differentiation and the wnt-signalling pathway, was observed. Analysis of gene expression using principal component analysis (PCA) showed a time-dependent track in untreated cells, describing the process of neural differentiation. Furthermore, $\mathrm{MeHg}$ was shown to induce deviation from the predefined differentiation track. The compound inhibited general development GO-terms and induced neural GO-terms over time. This system appears promising for studying compound effects on neural differentiation in a mechanistic approach.

\section{\&8}

\section{Introduction}

HE new European chemical safety regulation REACH (Regulation, Evaluation 1 and Authorization of Chemicals) requires many compounds to be tested. Current chemical hazard assessment for developmental toxicity is built upon globally harmonized OECD animal test guidelines, providing a structure for chemical risk assessment and in addition encouraging the development of alternative testing strategies. Under REACH guidelines, approximately $60 \%$ of all animals will be used for reproductive and developmental toxicity studies [12]. In order to reduce the number of experimental animals needed for developmental toxicity testing, cell based alternative test methods are being developed, such as embryonic stem cell tests (EST) studying multiple differentiation lineages [14, 17, 42, 142], the whole embryo culture (WEC) [14] and the zebrafish embryo test (ZET) [16, 78]. A common target for developmenta toxicity is the developing nervous system. Classical developmental toxicants, such as methylmercury $(\mathrm{MeHg})$, valproic acid and ethanol particularly target neural development resulting in neural tube defects (NTDs), craniofacia malformations, mental retardation and fetal alcohol syndrome [143-145]. To specifically examine known and unknown neurodevelopmental toxic compounds, in vitro test systems are being developed which mimic and/or partially represen nervous system development in vivo [41, 142, 146-148]. In particular, embryonic stem cells (ESC) provide a promising in vitro model for neural differentiation as they can be differentiated into multiple lineages of neural and other brainassociated cells $[52,103]$. In our previous study using mouse embryonic stem cells (ESC) [142] we designed an abbreviated 13-day neural differentiation protocol to examine the effects of neurodevelopmental toxic compounds. Using the classical neurodevelopmental toxicant $\mathrm{MeHg}$, we demonstrated the ability to examine the potential for neurodevelopmental toxicity based on morphological scoring of neural outgrowth from stem cell-derived embryoid bodies (EB).

While this test system and others show promise in being used for the prediction of neurodevelopmental toxicity, current criteria in assessing neurodevelopmental toxicity take into account limited endpoints of toxicity, such as cell morphology (neural outgrowth, cytotoxicity and migration) [149, 150], functional parameters determined by electric potential [147] or RNA transcripts of a small selected number of genes [41]. The complexity of the developing nervous system warrants a more comprehensive assessment at the molecular level of exposure effects by neurodevelopmental toxicants. Currently, emerging genomic technologies such as transcriptomics, which can be employed to assess effects on the whole-genome level are being implemented into in vitro and in vivo neurotoxicological studies 
$[82,151,152]$. The assessment of multiple endpoints both at the level of gene expression as well as considering molecular pathways is expected to provide a robust mechanistic approach to analyze and predict toxicity [83, 142, 153-155] To improve detection of developmental neurotoxicity and mechanistic understanding in the neural embryonic stem cell test (ESTn) model, a transcriptomics study was performed to assess the regular gene expression changes in unexposed differentiating ESC cultures. Furthermore, we studied the impact of the developmental toxicant $\mathrm{MeHg}$ on gene expression changes over time. During the past century, catastrophic MeHg poisonings in Japan and Irac have shown its neurodevelopmental toxicity [109, 111]. Since then, MeHg has become a well-characterized and widely used model compound for neurodevelopmental toxicity $[156,157]$.

In the present study, transcriptomics analysis over time of early differentia gene expression appeared to provide a robust method to monitor ultimate neural differentiation in the ESTn model. In addition, $\mathrm{MeHg}$ was shown to affect neurodevelopmental and proliferation-related gene pathways in association with affected neural differentiation, illustrating the added value of transcriptomics analysis of neural cell differentiation in vitro for the detection of developmental neurotoxicants and the study of developmental toxicity mechanisms of action.

\section{ఝో}

\section{Materials and Methods}

\section{Culture Media}

Complete medium (CM) contained Dulbecco's modified Eagle's medium (DMEM) (Gibco BRL, Gaithersburg, MD, USA) supplemented with $20 \%$ feta bovine serum (Hyclone, Logan, UT, USA), 1\% nonessential amino acids (Gibco BRL, Gaithersburg, MD, USA), 1\% penicillin/streptomycin (Gibco BRL, Gaithersburg, MD, USA), 2 mM L-glutamine (Gibco BRL, Gaithersburg, MD, USA) and $0.1 \mathrm{mM} \beta$-mercaptoethanol (Sigma-Aldrich, Zwijndrecht, The Netherlands). Low serum medium (LS), had the same composition as CM except that the serum percentage is $10 \%$. Insulin-transferrin-selenite-fibronectin medium (ITS) contained DMEM/Ham's nutrient mixture F12 (DMEM/ F12) medium (Gibco, BRL, Gaithersburg, MD, USA) supplemented with 0.2 $\mu \mathrm{g} / \mathrm{ml}$ bovine insulin (Sigma-Aldrich, Zwijndrecht, The Netherlands), 1\% Penicillin/Streptomycin (Gibco BRL, Gaithersburg, MD, USA), $2 \mathrm{mM}$ L-glutamine (Gibco BRL, Gaithersburg, MD, USA), $30 \mathrm{nM}$ sodium selenite (Sigma-Aldrich, Zwijndrecht, The Netherlands), $50 \mu \mathrm{g} / \mathrm{ml}$ apo-transferrin
Sigma-Aldrich, Zwijndrecht, The Netherlands) and $2.5 \mu \mathrm{g} / \mathrm{ml}$ fibronectin (nvitrogen, Carlsbad, CA, USA). N2 medium contained DMEM/F12 medium (Gibco, BRL, Gaithersburg, MD, USA) supplemented with $0.2 \mu \mathrm{g} / \mathrm{ml}$ bovine insulin (Sigma-Aldrich, Zwijndrecht, The Netherlands), 1\% penicillin/ streptomycin (Gibco BRL, Gaithersburg, MD, USA), $30 \mathrm{nM}$ sodium selenit (Sigma-Aldrich, Zwijndrecht, The Netherlands), $50 \mu \mathrm{g} / \mathrm{ml}$ apo-transferrin (Sigma-Aldrich, Zwijndrecht, The Netherlands), $20 \mathrm{nM}$ progesteron (SigmaAldrich, Zwijndrecht, The Netherlands) and $100 \mu \mathrm{M}$ putrescine (SigmaAldrich, Zwijndrecht, The Netherlands).

\section{Embryonic Stem Cell Culture}

Murine embryonic stem cells (ESC) (ES-D3, ATCC, Rockville, MD, USA were routinely sub-cultured every $2-3$ days and grown as a monolayer in CM supplemented with leukemia inhibiting factor (LIF) (Chemicon, Temecula, CA, USA) at a final concentration of 1000 units $/ \mathrm{ml}$. The cells were maintained in a humidified atmosphere at $37^{\circ} \mathrm{C}$ and $5 \% \mathrm{CO}_{2}$.

\section{Neural Differentiation Culture}

Differentiation of murine ESC into the neural lineage was carried out as described earlier [142]. In brief, stem cell suspensions $\left(3.75 \times 10^{4}\right.$ cells $\left./ \mathrm{ml}\right)$ were placed on ice before the initiation of the culture. Drops $(20 \mu 1)$ containing 750 cells in CM were placed onto the inner side of the lid of a $90 \mathrm{~cm}$ Petri dish filled with phosphate-buffered saline (PBS) (Gibco BRL, Gaithersburg, MD) and incubated at $37{ }^{\circ} \mathrm{C}, 90 \%$ relative humidity and $5 \% \mathrm{CO}_{2}$. After 3 days of hanging drop culture embryoid bodies (EB) had formed and were subsequently transferred to $60 \mathrm{~mm}$ bacterial Petri dishes (Greiner Bio-one, Frickenhausen, Germany) containing CM supplemented with $0.5 \mu \mathrm{M}$ retinoic acid (RA) (Sigma-Aldrich, Zwijndrecht, The Netherlands). On day 5, EB were plated on aminin (Roche, Basel, Switzerland) coated dishes (Corning Incorporated, Corning, NY, USA) in LS medium supplemented with $2.5 \mu \mathrm{g} / \mathrm{ml}$ fibronectin (Invitrogen, Carlsbad, CA, USA). One day later, on day 6, the LS medium was replaced by ITS medium. On day 7, EB were washed with PBS and incubated in cell dissociation buffer (Gibco BRL, Gaithersburg, MD, USA) for 3 minutes. Then EB were carefully detached from each Petri dish without dissociating the EB and the entire content was replated on poly-L-ornithine (Sigma-Aldrich, Zwijndrecht, The Netherlands) and laminin coated dish in N2 medium supplemented with $10 \mathrm{ng} / \mathrm{ml}$ basic fibroblast growth factor (bFGF) (Srtathmann-Biotec AG, Englewood, CO, USA). The N2 medium supplemented with bFGF was replaced every other day for 7 days (Figure 1). 


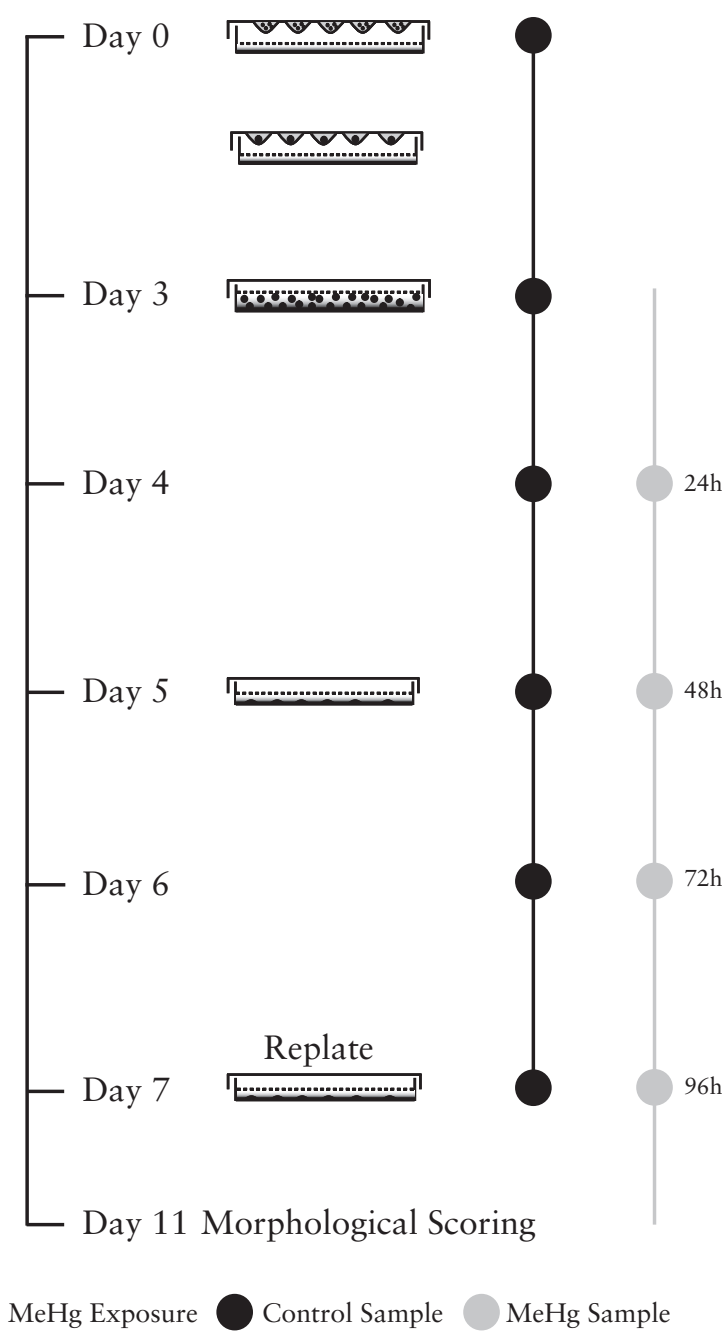

Figure 1 Hanging drops containing ESC were made on day 0. Single cells formed embryoid bodies (EB). On day 3, the EB were transferred to suspension medium supplemented with $0.5 \mu \mathrm{M}$ all-trans-retinoic acid (RA). On day 5, EB were plated on laminin coated dishes and kept in medium containing $10 \%$ serum. On day 6 , the medium was replaced with a serum-free medium (ITS). On day 7, EB were detached from the dish and replated on PLO/laminin coated dishes and N2 medium was added. On day 11, morphological scoring for neural outgrowth was performed. $\mathrm{MeH}$ treatment was started on day 3 of the protocol and treated samples were collected after $24,48,72$ and $96 \mathrm{~h}$ exposure ( $\mathrm{n}=8$ per group). Control samples were collected on days $0,3,4,5,6$ and 7 ( $\mathrm{n}=8$ per group).

\section{Treatment and morphological scoring for effects on neural} outgrowth

Methylmercury chloride (MeHg) (CAS number 115-09-3, Sigma-Aldrich, Zwijndrecht, The Netherlands) was diluted in dimethyl sulfoxide (DMSO) and added to the medium to final concentrations in culture of $25 \mathrm{nM} \mathrm{MeHg}$ and control EB were treated with $0.01 \%$ DMSO as described earlier from day 3 of the protocol until day 11 [142]. This concentration of $\mathrm{MeHg}$ did not affect cell viability in the model. Effects were determined by assessing of the morphological extent of neural outgrowth, at day 11, observed using an IX51 inverted microscope (Olympus, Zoeterwoude, The Netherlands) with CellD software (Olympus, Zoeterwoude, The Netherlands). Morphological neural outgrowth was scored as $<75 \%$ or $\geq 75 \%$ of the neural corona surrounding each EB irrespective of the distance of outgrowth from the EB. Parallel cultures were harvested for gene expression analysis at earlier time points.

\section{RNA isolation and whole-genome expression profiling}

Control differentiation cultures were harvested on culture days $0,3,4,5,6$ and 7 (eight replicates per group). MeHg exposed differentiation cultures were exposed from day 3 onwards and harvested on day 4, 5, 6 and 7 (24, 48, 72 and 96h exposure, respectively; eight replicates per group) (Figure 1). Cells ( $\sim 40$ to $50 \mathrm{~EB} / \mathrm{sample}$ ) were directly collected in RNA Protect (Qiagen Benelux, Venlo, The Netherlands) to stabilize RNA, and total RNA was purified using the Qiagen RNEasy Plus Mini Kit (Qiagen Benelux, Venlo, The Netherlands) following manufacturer's instructions. RNA quantity was determined using the NanoDrop Spectrophotometer (Isogen Lifescience, de Meern, The Netherlands). RNA integrity was assessed on the 2100 Bioanalyzer (Agilent, Santa Clara, CA, USA) using the RNA 6000 Nano Chip Kit (Agilent, Santa Clara, CA, USA), and good quality RNA was used for gene expression analysis (RNA integrity number $(\mathrm{RIN})>8.0$ ). Labeled aRNA target was generated by using the Affymetrix genechip (Affymetrix, Santa Clara, CA, US) (3'IVT express kit $4 \times 24$ reactions; P/N 901225) according to the instructions in the user's manual. Briefly, $250 \mathrm{ng}$ of each total RNA sample and also controls were used to prepare first-strand complementary DNA (cDNA). After making the complementary second strand, the double stranded cDNA is amplified by in vitro transcription (IVT). During this IVT reaction a biotinylated nucleotide analog is incorporated in the aRNA. This IVT reaction was followed by aRNA purification with magnetic beads and fragmentation $(15 \mu \mathrm{g})$ of each aRNA sample. The whole process from first-strand cDNA synthesis till fragmentation of the aRNA was performed by the Xiril Neon 150 robotic system (GC-Biotech, Alphen aan den Rijn, The Netherlands). 
After fragmentation a $250 \mu \mathrm{l}$ hybridization cocktail with $12,5 \mu \mathrm{g}$ of fragmented aRNA was prepared. $200 \mu \mathrm{l}$ of this cocktail (10 $\mu \mathrm{g}$ aRNA target) was applied to the Mouse Genome 4302.0 arrays (P/N 900497, Affymetrix, Santa Clara, CA, US) and hybridized for 16 hours at $45^{\circ} \mathrm{C}$ in a Genechip Hybridization Oven 640. After hybridization the arrays were washed and stained with a Genechip Fluidics Station 450 using the Affymetrix genechip hybridization wash and stain kit (P/N 900720). Washed and stained arrays were scanned using the genechip scanner 3000 from Affymetrix. Hybridization was performed at Affymetrix (Santa Clara, CA, US).

\section{Data Analysis and statistics}

Affymetrix CEL files were normalized using the Robust Multichip Average (RMA) algorithm [158] using RMAexpress [159]. For probe to gene mapping, a custom Chip Description File (CDF) was used according to the assembly by de Leeuw et al. [160] (http://mad-db.science.uva.nl/ wdeleeuw/HybridAnnot/ version6.html). Of the hybrid probe-set definitions included in the custom annotation, 16,331 probe sets defined by the Brainarray custom CDF version $11 \mathrm{http}: / /$ brainarray.mbni.med.umich.edu/Brainarray/Database/CustomCDF [161] and 4648 additional probe sets defined by Affymetrix chip annotation 26 were used in further analyses, giving a total of 20,979 probe sets. Probe sets for Affymetrix internal controls or probe sets that did not correspond to an Entrez Gene ID were not used in further analyses. Statistical analyses were carried out using the R statistical software environment (http://www.R-project. org) using log-transformed values. For each gene, maximal fold ratios (FC) in gene expression between the experimental groups were determined by comparing the average normalized signal values per group and were calculated as the maximum/minimum ratio. For the time series, expression of a gene was compared to the median expression of all samples. For MeHg toxicity effects, the treated samples were compared to the time matched controls. Genes differentially expressed between any of the experimental groups were identified by a one-way ANOVA on the normalized data. A significance threshold of $\mathrm{p}<0.001$ and $\mathrm{FC}>2$ were used to select genes that were significantly differentially expressed when ESC differentiate over time. A $\mathrm{p}<0.001$ with $\mathrm{FC}>1$ restriction was applied for the identification of significantly differentially expressed genes caused by $\mathrm{MeHg}$ exposure. Hierarchical clustering of genes significantly differentially expressed over time in the neural differentiation cultures was performed in GeneMaths XT (Applied Maths, Sint-Martens-Latem, Belgium) using Euclidean distance and Ward linkage. Functional annotation and enrichment for Gene Ontology (GO) biological processes and KEGG pathways were studied using the tools on the DAVID Web site (http://david.abcc.ncifcrf. gov/) [162]. Terms with a $\mathrm{p}<10^{-4}$ and a Functional Annotation Clustering Enrichment Score $>3$ were considered enriched, and if terms clustered together, the most significant functional term within that cluster was used. For pathway-level analysis, each gene was corrected for its average across all time points, after which Tox-profiler [99] was used to score the difference between the mean expression level of predefined groups of genes (e.g. GO-terms or literature based gene sets) and that of the remainder of the 20,979 probe sets defined earlier. Gene sets were significantly regulated if $\mathrm{T}>3.5$ and $\mathrm{E}>0.05$. The T-value is the value obtained by a T-test between the expression change for a defined set of genes versus all other genes. The corresponding formula and other calculation details can be found in Boorsma et al. [99]. The E-value is the associated two-tailed P-value with Bonferroni correction for the number of gene sets tested. Literature gene sets were derived from Kuegler $e t$ al. (mouse ESC and neural stem cell (NSC) gene sets) [62] and the GNF1M Gene Atlas data set described by Su et al. [163] (downloaded from GEO accession number GSE1133) describing mouse tissue-specific gene sets. To correct for overlap between tissue-specific gene sets, forward selection was performed. In short, the gene set with the highest absolute T-value is used and the mean contribution of each gene in the set is subtracted from all genes in the gene set. Then a new T-value is calculated with the changed values and the same process is repeated until no significant gene sets are found. The gene expression over time and the effect of $\mathrm{MeHg}$ on gene expression was further studied by means of the differentiation track algorithm, an approach based on principal componen analysis (PCA) [86]. In this analysis, a principal component is defined as a mathematically derived combination of genes and their expression characteristics which can be used to depict part of the process observed. A number of principal components that are mutually independent can be derived which in combination describe the process under study. For the differentiation track algorithm, at first a gene list was defined, which comprised the genes that were identified to be significantly differentially expressed among ESC differentiation sampled at different stages over time. Next, PCA analysis was performed using these genes using $\mathrm{R}$. We connected a line between the average array gen expression at days $0,3,4,5,6$ and 7 on our PCA plot to display the continuous representation of the ESC differentiation over time based on the dynamics of gene expression. This curve was defined as the 'differentiation track' over time. For describing the effects of $\mathrm{MeHg}$ on the track at a particular day, a comparison was made with a differentiation track derived on the basis of genes showing differential expression between the day before, the same day, and the day after the time point analyzed. Limiting the analysis to genes that show time-related expression in the protocol avoided the contribution of genes not 
regulated during neural development in the protocol. Coordinates along the first and second principal components were calculated for each sample. Deviation of compound-exposed cultures from the differentiation track was analyzed by applying a Hotelling t-test to these coordinates compared with those of time-matched control cultures. Significant deviation from the differentiation track $(\mathrm{p}<0.05)$ was considered characteristic for developmenta toxicants. Genes significantly changed $(\mathrm{p}<0.001, \mathrm{FC}>2.0)$ in the period of the 3 days surrounding the specific treatment day of interest were used to set up the track and the gene expression of the $\mathrm{MeHg}$ samples was placed in the track

\section{@ో}

\section{Results}

ESC (neural) differentiation is accompanied by gene expression changes over time

We identified 4393 genes which were significantly differentially expressed $(\mathrm{p}<0.001$ and $\mathrm{FC}>2)$ in control cultures across time (days 0, 3, 4, 5, 6 and 7). Eight gene clusters $(\mathrm{A}-\mathrm{H})$ were defined by hierarchical clustering (Euclidean distance, ward linkage) on the basis of expression dynamics over time (Figure $2 \mathrm{~A}$ and $2 \mathrm{~B}$ ). Cluster A showed a high gene expression early (day 0 ) and later in time (days 6 and 7), but low expression on d3-5. Due to the small amount of genes present in this cluster, no significant $\left(\mathrm{p}<1^{*} 10^{-4}\right) \mathrm{GO}$-terms were found. Genes in cluster B were mainly increased at early time points (day 0 and 3). GO-terms enriched within this cluster were associated with transcription (nucleolus, rRNA, tRNA, and DNA metabolic process), cell metabolism (mitochondrion), and pluripotency (blastocyst formation and trophectoderm cell differentiation). Highly upregulated genes in this cluster (FC $>16)$ included the pluripotency-related genes Pou5f1 and Nodal [164, 165]. In cluster C, genes were mainly increased on day 3 and in GO-terms involved in early development (pattern specification, embryonic morphogenesis, and blastocyst-, mesoderm- and endoderm-related clusters) and cell migration. Cluster D was increased on day 4 and 5. Due to the small amount of genes present in set $\mathrm{D}$, no significant $\left(\mathrm{p}<1^{*} 10^{-4}\right) \mathrm{GO}$-terms were found. In cluster $\mathrm{E}$, genes were increased from day 4 onwards and expression increased over time. This cluster was enriched for the Wnt-signaling pathway and TGF $\beta$-signaling pathway, both important in development. In addition, a broad range of developmenta GO-terms were enriched (neuron development, tube development, skeletal development, vasculature development, limb development and urogenital system development). Cluster F genes were mainly increased on days 6 and 7 and genes in cluster $G$ were mainly increased over time from days 4, 5, 6 and 7 and relatively low on day 0 . Cluster $\mathrm{H}$ genes were low expressed on days 0 and 3 and highly increased on days 5,6 and most on day 7. A high overlap was observed for GO-terms enriched in clusters F, G and $\mathrm{H}$, which consisted mainly of neural associated GO-terms (neuron differentiation, -fate commitment andmigration) and other development-related GO-terms (pattern specification, skeletal development, inner ear development and eye development). In the enriched neuron differentiation GO-term, many markers known to play a critical role in neuronal development were highly $(\mathrm{FC}>16)$ induced, such as Pax6, Tubb3 and Nefl [166, 167].

ESC (neural) differentiation cultures express tissue specific gene sets Gene sets for embryonic day 8.5, 9.5 and 10.5 extracted from GEO, and mouse tissue-specific gene sets were analyzed in Tox-profiler to determine the most prominent tissues developing over time in our neural differentiation protocol (Figure 2C). Embryonic day 8.5 showed an increase in gene expression from day 4 onwards, peaking on day 5 and a decrease in gene expression from day 6 onwards. Embryonic_day_9.5 showed a similar expression profile compared to embryonic_day_8.5, but with a higher T-value and significance. The firs significant increase for the embryonic_day_10.5 set was observed on day 5 , and expression further increased on days 6 and 7. Over time, gene sets representing very early development (blastocyst and oocyte) were induced on day 0 and their expression diminished over time. Furthermore, sets important during the post implantation period (placenta and umbilical cord) were increased from day 5 onwards. Gene sets of tissues derived from the ectoderma lineage (different regions of the brain and spinal cord) were starting to increase on day 6 and were strongly increased on day 7. Regarding the endoderma derived tissues, expression for lung was decreased significantly only on day 4 and gene expression for the small intestine and salivary gland was not significantly regulated over time. Furthermore, the gene sets describing mesodermal derived kidney and testis were reduced from day 6 onwards.

Neural differentiation culture shows a time dependent change in PCA PCA was performed on control samples (days $0,3,4,5,6$ and 7 ) using the 4393 genes differentially expressed over time (Figure 2D). 76\% of the variance could be assigned to the first $(60 \%)$ and second $(16 \%)$ principal components of the PCA. Groups of each time point clustered together, forming a chronological representation of neurological differentiation, which we will refer to as the 'differentiation track' [142]. 


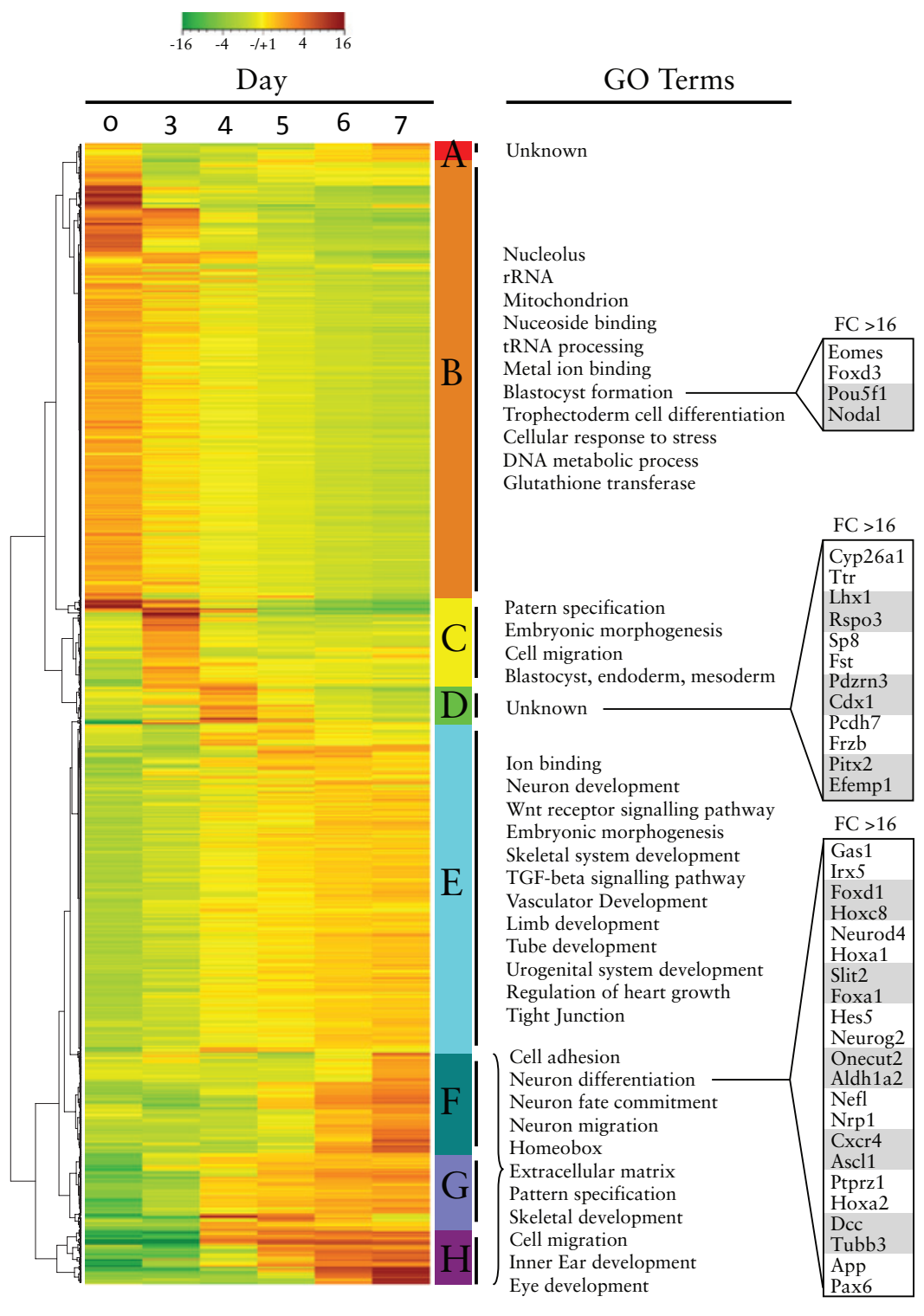

Figure 2A Gene expression describing differentiation over time in the ESTn. Heatmap of all genes differentially expressed over time ( $p>0.001 ; \mathrm{FC}>2.0)$ in unexposed differentiation cultures. Each row represents a single gene. Eight clusters were defined based on comparable expression profiles over time. For each cluster, GO-terms enriched for that cluster are indicated. GO and KEGG terms enriched for cluster F, G and $\mathrm{H}$ were comparable.

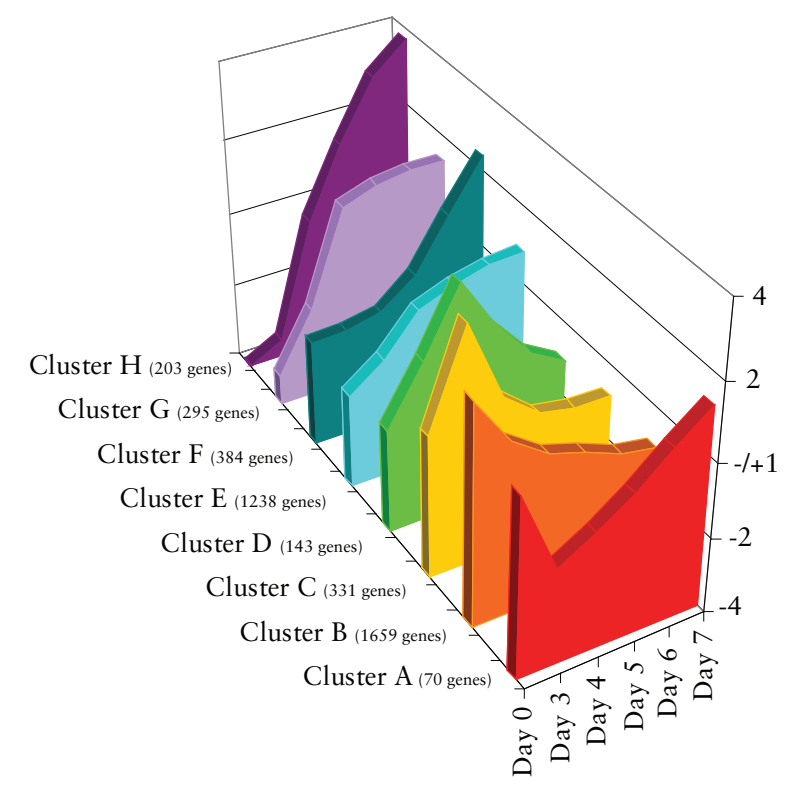

Figure 2B Mean expression values of genes differentially expressed over time in each cluster $(\mathrm{A}-\mathrm{H})$.

\section{Morphological Scoring shows MeHg effects}

As described earlier [142] $\mathrm{MeHg}$ (25 nM, exposure from day 3-11) resulted in a significant decrease in neural outgrowth as compared to concurrent controls. MeHg-exposed samples showed a $63 \%( \pm 10 \%)$ decrease of EB with $>75 \%$ outgrowth around the EB.

$\mathrm{MeHg}$ increases expression of neural development related genes Gene expression changes in cultures treated with $\mathrm{MeHg}$ (treatment from day 3 onwards for $24 \mathrm{~h}, 48 \mathrm{~h}, 72 \mathrm{~h}$ and $96 \mathrm{~h}$ ) were compared to their time matched control on days 4, 5, 6 and 7 by a one-way ANOVA ( $\mathrm{p}<0.001$ and $\mathrm{FC}>1.0)$. After $24 \mathrm{~h}$ treatment, 490 genes were significantly regulated, after $48 \mathrm{~h}$ treatment, 6 genes, after $72 \mathrm{~h}, 29$ genes and after $96 \mathrm{~h}, 97$ genes were significantly regulated. In total, 611 genes were significantly differentially regulated by $\mathrm{MeHg}$ over time. Clustering of these genes showed predominantly a group downregulated over time and a group upregulated over time (Figure 3A). Analysis using DAVID showed the downregulated group had significant changes in transcription- and embryonic development-related terms. The 
T-values of expression of BioGPS tissue gene sets over time on days
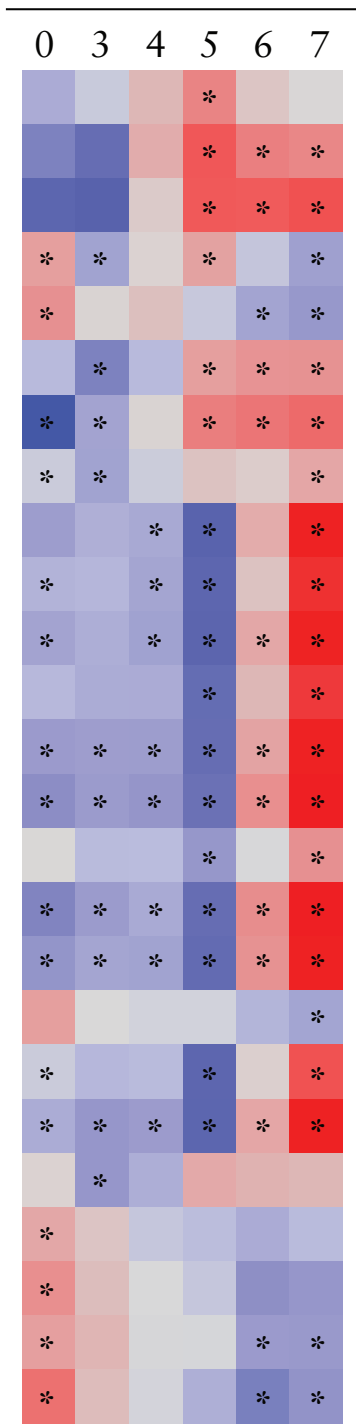

Embryonic day 8.5

mbryonic day 9.5

Embryonic day 10.5

Blastocyst

Oocyte

Placenta

Umbilical Cord

Uterus

Amygdala

Cerebellum

Cerebral Cortex

Dorsal Root Ganglia

Frontal Cortex

Hypothalamus

Medial Olfactory Epitheliu

Olfactory Bulb

Preoptic

Snout Epidermis

Spinal Chord, Upper

Substantia Nigra

Lung

Salivary Gland

Small Intestine

Kidney

Testis

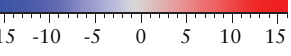

Embryonic Day

Very Early

Development

Post Implantation

Ectoderm

Mesoderm

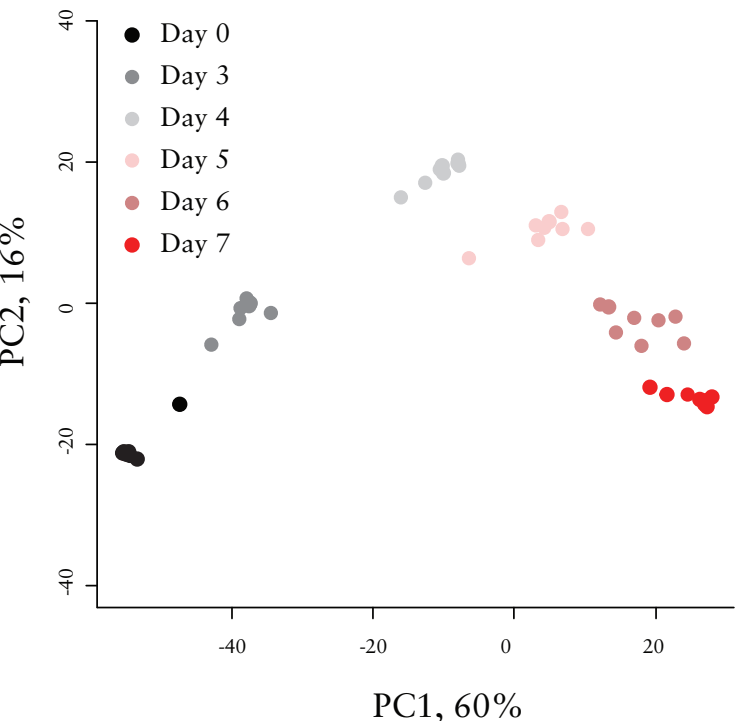

Figure 2D Principal component analysis (PCA) plot of control (colored full circles) differentiation cultures over time The PCA is based on the 4339 genes regulated over time ( $\mathrm{p}>0.001$ and $\mathrm{FC}>2)$.

upregulated group showed significant enrichment for cell motion and neuron development terms.

\section{$\mathrm{MeHg}$ increases late differentiation genes and decreases early}

\section{differentiation genes}

Gene set clusters derived from neural differentiation over time (Figure 2A, clusters A-H) and clusters describing mouse ESC and neural stem cell (NSC) gene expression [62] were used to study the effect of $\mathrm{MeHg}$ with Tox-profiler (Figure 3B). A significant increase in gene expression by $\mathrm{MeHg}$ at most time points was observed in the late time clusters (E, F, G and $\mathrm{H}$ ) and in the NSC set. In set A, only on day 6 a small significant increase in expression was observed. In contrast, the early time clusters $(\mathrm{B}, \mathrm{C}$ and $\mathrm{D})$ and the mESC set were significantly downregulated by $\mathrm{MeHg}$ at most time points.

Figure 2C Heatmap of T-values for tissue-specific gene sets derived from GEO over time Values were derived using T-profiler and sets were selected using forward selection. $\mathrm{T}>3.5$ and $\mathrm{E}>0.05$. 
$\mathrm{MeHg}$ treated samples

$$
\text { compared to }
$$

day matched control

GO Terms
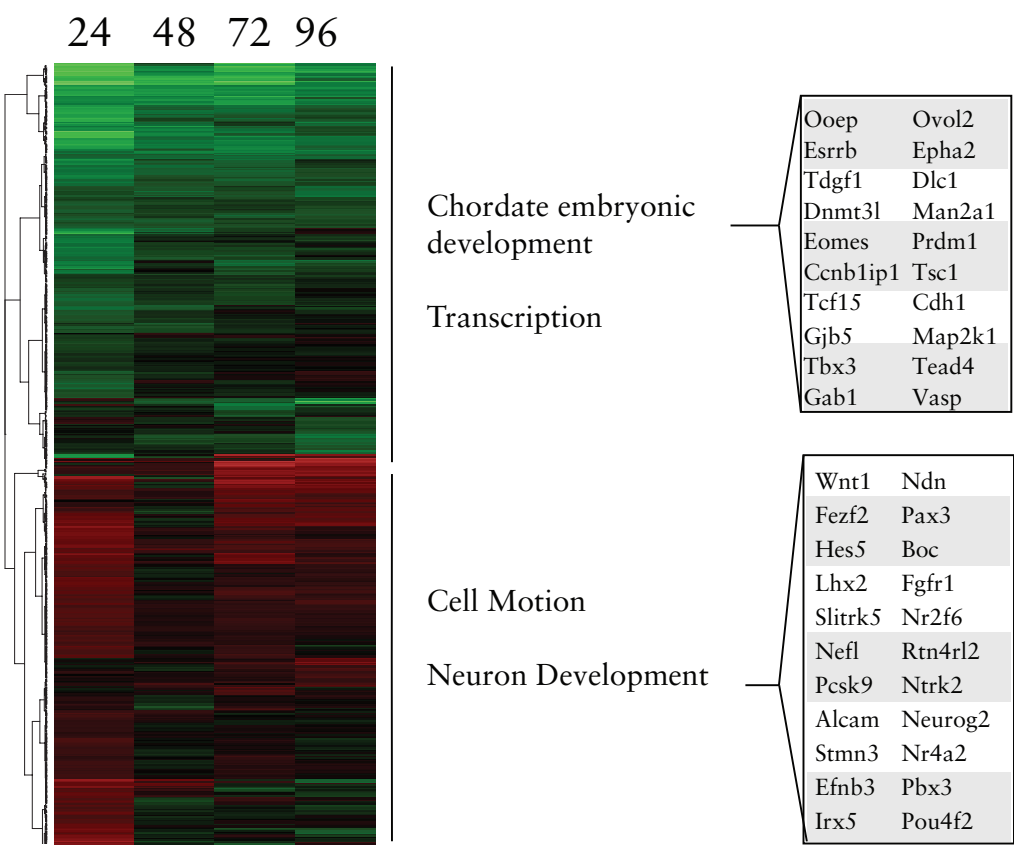

Cell Motion $\quad$\begin{tabular}{|ll|}
\hline Wnt1 & Ndn \\
Fezf2 & Pax3 \\
Hes5 & Boc \\
Lhx2 & Fgfr1 \\
Slitrk5 & Nr2f6 \\
Nefl & Rtn4rl2 \\
Pcsk9 & Ntrk2 \\
Alcam & Neurog2 \\
Stmn3 & Nr4a2 \\
Enfb3 & Pbx3 \\
Irx5 & Pou4f2 \\
\hline
\end{tabular}

$-\frac{1}{-3-1}$ after hours treatment

$\mathrm{MeHg}$ compared to day matched control in T-values after hours treatment

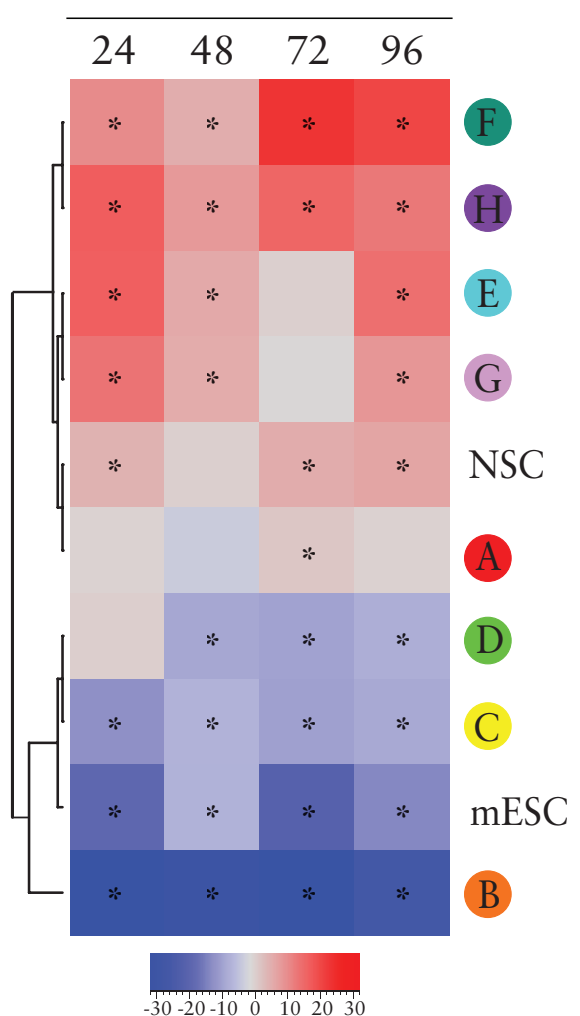

Figure 3B Effects of MeHg on gene expression in the ESTn. Heatmap of T-values for clusters derived from differentiation over time due to $\mathrm{MeHg}$ compared to time matched controls. Values were derived using T-profiler. ${ }^{*} \mathrm{~T}>3.5$ and $\mathrm{E}>0.05$.

es differentially expressed by $\mathrm{MeHg}$ compared to time matched controls ( $p>0.00$ FC>1.0). Each row represents a single gene.

\section{$\mathrm{MeHg}$ increases neurectodermal gene sets and decreases}

developmental, mesodermal and endodermal derived tissue gene sets Mouse tissue-specific gene sets were analyzed in Tox-profiler to determine the most important tissues affected by $\mathrm{MeHg}$ compared to time matched controls (Figure 3C). Gene sets associated with very early development were down regulated by $\mathrm{MeHg}$ as was the placenta gene set. Gene sets of specific embryonic days showed a decrease in average gene expression by $\mathrm{MeHg}$ in early embryonic days (6.5 and 7.5). The embryonic_day_8.5 only showed a significant decrease on days 6 and 7 and embryonic_day_10.5 showed a significant increase on day 5. No significant changes were observed for the embryonic_day_9.5 gene set (data not shown). In contrast, gene sets of tissues related to neuroectoderm were significantly increased by $\mathrm{MeHg}$ on days 6 and 7 and in a few cases also on day 4. Non neural ectodermal gene sets (retina and snout epidermis) showed no differences or a small decrease. Expression of mesodermal and endodermal gene sets was predominantly decreased on day 7 . 
Effect of $\mathrm{MeHg}$ on BioGPS tissue gene set expression in T-values after hours treatment

\begin{tabular}{|c|c|c|c|c|c|}
\hline 24 & 48 & 72 & 96 & & \\
\hline$*$ & $*$ & $*$ & $*$ & Embryonic Day 6.5 & \multirow{4}{*}{ Embryonic Day } \\
\hline$*$ & $*$ & $*$ & $*$ & Embryonic Day 7.5 & \\
\hline & & $*$ & $*$ & Embryonic Day 8.5 & \\
\hline & $*$ & & 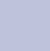 & Embryonic Day 10.5 & \\
\hline$*$ & $*$ & $*$ & $*$ & Blastocyst & \multirow[b]{2}{*}{ Very Early Development } \\
\hline$*$ & $*$ & $*$ & & Oocyte & \\
\hline * & & $*$ & $*$ & Placenta & \multirow{2}{*}{ Post Implantation } \\
\hline$*$ & & * & * & Umbilical Cord & \\
\hline \multirow[t]{2}{*}{ * } & & $*$ & $*$ & Cerebral Cortex & \multirow{8}{*}{ Ectoderm } \\
\hline & & $*$ & * & Dorsal Root Ganglia & \\
\hline$*$ & & $*$ & $*$ & Frontal Cortex & \\
\hline & & * & & Medial Olfactory Epithelium & \\
\hline * & & $*$ & $*$ & Olfactory Bulb & \\
\hline \multirow[t]{6}{*}{$*$} & & $*$ & $*$ & Preoptic & \\
\hline & $*$ & & & Retina & \\
\hline & $*$ & & $*$ & Snout Epidermis & \\
\hline & $*$ & & $*$ & Bladder & \multirow{4}{*}{ Endoderm } \\
\hline & & & $*$ & Liver & \\
\hline & $*$ & & $*$ & Lung & \\
\hline \multirow[t]{3}{*}{$*$} & $*$ & & $*$ & Small Intestine & \\
\hline & $*$ & & $*$ & Kidney & \multirow{2}{*}{ Mesoderm } \\
\hline & & & $*$ & Testis & \\
\hline
\end{tabular}

Figure 3C Effects of MeHg on gene expression in the ESTn. Heatmap of T-values for tissue-specific gene sets derived from $\mathrm{GEO}$ due to $\mathrm{MeHg}$ compared to time--matched controls. Values were derived using T-profiler. ${ }^{*} \mathrm{~T}>3.5$ and $\mathrm{E}>0.05$.

\section{$\mathrm{MeHg}$ exposed cultures deviate over time from the neural}

\section{differentiation track}

The effect of MeHg on gene expression was further studied using PCA by mean of the differentiation track algorithm. Genes significantly regulated in controls "day 3/4/5" (1509 genes), "day 4/5/6" (782 genes) and "day 5/6/7" (586 genes) were compared to gene expression after exposure to $\mathrm{MeHg}$ as assessed on day 4 , day 5 and day 6 , respectively. $\mathrm{MeHg}$ treatment measured on day 4 deviated significantly from the track $(\mathrm{p}=0.0008)$ (Figure $4 \mathrm{~A})$, but $\mathrm{MeHg}$ measured on day $5(\mathrm{p}=0.19)$ (Figure $4 \mathrm{~B})$ and day $6(\mathrm{p}=0.08)$ (Figure $4 \mathrm{C}$ ) were not significantly different as compared to their control 3-day differentiation track.

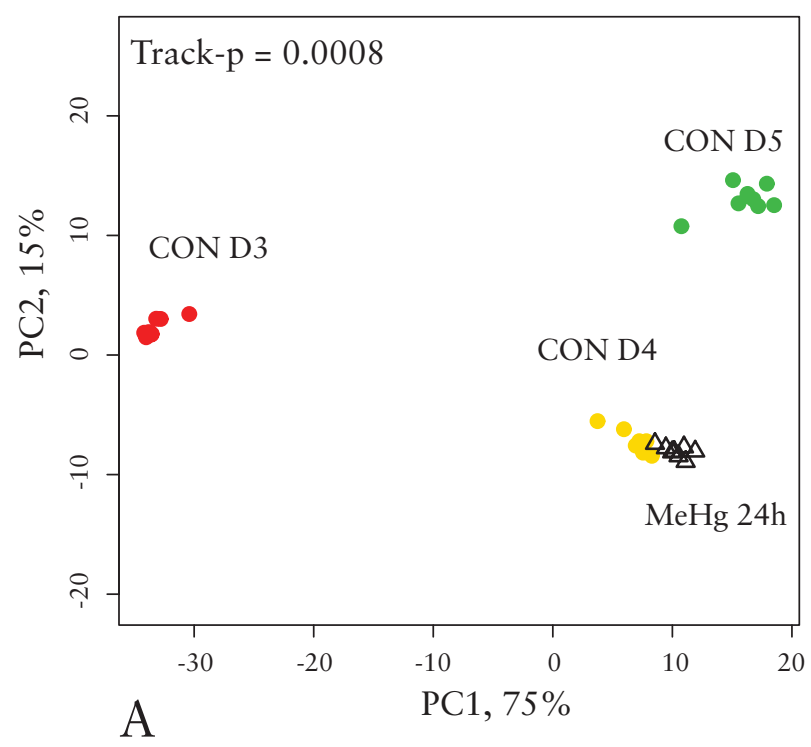

Figure 4 PCA plots of control (colored full circles) over three time points and $25 \mathrm{nM}$ $\mathrm{MeHg}$ exposed (black open triangles) on the middle time point. The PCA are based on the regulated genes over the three time points within the plot ( $p>0.001$ and $\mathrm{FC}>2)$. The track-p describes the difference between the $\mathrm{MeHg}$ group compared to the time-matched control. A) Control day 3 (red), day 4 (gold), day 5 (green) and $\mathrm{MeHg}$ on day 4 (black) B) control day 4 (red), day 5 (gold), day 6 (green) and $\mathrm{MeHg}$ on day 5 (black); C) contro day 5 (red), day 6 (gold), day 7 (green) and $\mathrm{MeHg}$ on day 6 (black). 

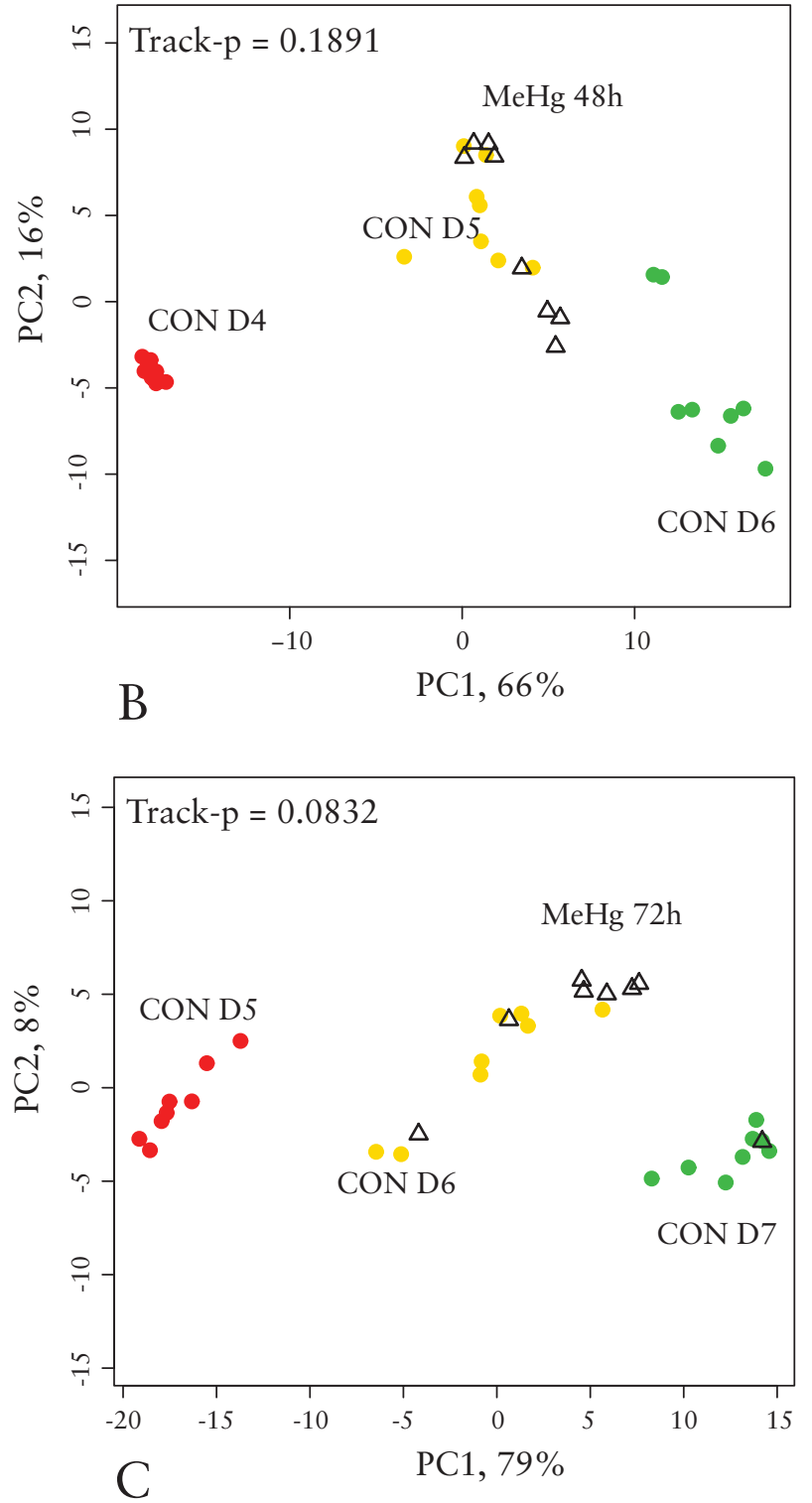

Figure 4 Continued

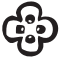

\section{Discussion}

ESC neural differentiation and gene expression changes over time Transcriptomics analyses were instrumental in the description of the neural differentiation process of ESC (Figure $2 \mathrm{AB}$ ), showing a reduction of pluripotency and an induction of neural differentiation-related gene sets over time. In addition, two gene clusters (C and D) were found to be transiently regulated at day 3 and 4 of the ESTn protocol, respectively. Gene clusters behaving similarly were observed in gene expression profiles over time for the cardiac embryonic stem cell test (ESTc) [142]. A number of the top regulated genes (FC>16) in ESTn gene cluster $\mathrm{D}$ are involved in RA metabolism and brain development, such as Cyp26a1 [168] It has been suggested that genes in these clusters could be important in early stages of differentiation [142]. The differentiation processes observed over time were reminiscent of developmental pathways observed in the embryo in a similar time-dependency (Figure 2C), and showed clear germ line specificity for induction of neurodevelopmental pathways. Furthermore, the differentiation process within the ESTn over time could be visualized in a two-dimensional PCA plot (Figure 2D). Our results support the observations of neuronal differentiation and the loss of pluripotency in the ESTn over time described earlier [142] and provide a more detailed mechanistic assessment of the dynamic changes underlying neural development in the model. Previously, genomics studies using a broad variety of mouse ESC neural differentiation culture techniques and time points [80, 169, 170] described similar changes in expression of genes as observed in our study. For instance, Abranches et al. [169] described comparable regulated genes at time points which correspond to the ESTn protocol (early differentiation genes Frzb, Id 2 and Lrp2, late differentiation genes Pax6, Neurog1, Nhlb1, Notch1 and $L x 9)$. Furthermore, gene expression profiles in our study correspond to published mouse in vivo developmental gene sets describing early neural differentiation. Mitiku and Baker [171] described the mouse transcriptome of gastrulation through organogenesis from E6.25 to E9.0. One of the specific clusters they identified was highly enriched for neural development-related GO-terms. The expression profile of the clusters F-H in our study correspond with the gene expression profile found in the cluster described by Mitiku and Baker (72 of 227 genes), with enriched GO-terms for neural development, neuron migration, axiogenesis and axon guidance, among others. Analysis of the cluster defined by Mitiku and Baker in Tox-profiler on our time data showed an increase in expression from day 4 to day 7. Therefore, ESTn transcriptomics shows an extensive correlation with morphological neurodevelopment while providing a more detailed assessment of dynamic changes in gene expression and the associated functional processes over time. 


\section{$\mathrm{MeHg}$ induced gene expression changes over time in neural} differentiating ESC

$\mathrm{MeHg}$ exposure resulted in down-regulation of transcription- and developmentrelated genes and in up-regulation of neurodevelopment and cell motion-related gene sets (Figure 3AB). MeHg retarded embryonic development as observed by stage-specific gene expression, but specifically increased expression of neura differentiation gene sets (Figure 3C). The relatively small gene expression changes early in the protocol as compared to the more robust morphological effects observed at the end of the protocol is probably related to the difference in timing of both parameter types. The molecular mechanisms behind $\mathrm{MeHg}$ neurodevelopmental toxicity have currently not been identified, but in vivo and in vitro studies have suggested mechanisms involving oxidative stress, cell cycle regulatory proteins, protein phosphorylation and intracellular calcium homeostasis [144, 172]. In our previous study focusing on protein and cellular effects, $\mathrm{MeHg}$ inhibited mESC neurite growth and migration, and at higher concentrations increased the neural precursor marker protein nestin [142]. In another study, $\mathrm{MeHg}$ was shown to decrease the expression of the neuron-specific cytoskeletal protein Mtap2 gene expression after 14 days of mESC neural differentiation at a concentration of $100 \mathrm{nM}$ [41]. Studies describing $\mathrm{MeHg}$ developmental toxicity using genomics in mouse embryos undergoing neurulation [151, 152] and the rat whole embryo culture (WEC) [82] showed up-regulation of early neural differentiation gene sets associated with neurodevelopmental malformations observed subsequently. In vivo studies showed that $8 \mathrm{~h}$ and $12 \mathrm{~h}$ after $\mathrm{MeH}$ exposure in utero, neural developmental pathways, such as the wnt signaling pathway [152] were upregulated at dose levels which were associated with neural tube defects (NTDs) and other malformations [173]. In the rat WEC study [82], $\mathrm{MeHg}$ induced neural gene expression up-regulation at $4 \mathrm{~h}$ in association with multiple developmental defects at 48h (NTDs and effects on yolk sac, branchial bar, eye, mandibular, heart and limb). These observations from three different models indicate that up-regulation of neuro-developmental genes at an early time point was related to impaired morphological development at a later time point.

Differentiation track analysis of neuro-developmental toxicity effects in ESTn Specific manifestations of developmental toxicity at the morphological level can be caused by diverse effects on the gene expression level, representing different mechanisms of toxic action [151]. Studying individual genes or proteins in stem cell differentiation assays gives only limited information about mechanisms of action of compounds [41, 142, 174]. Larger sets of functionally related genes may help to further clarify mechanisms of neurodevelopmental toxicity. In previous studies using the ESTc, van Dartel et al. $[40,83,142,155]$ used the differentiation track method to determine compound toxicity in a tempora manner. In the present study, we used the time related differentiation track method [142] to study gene expression over time. The effects of $\mathrm{MeHg}$ on cell differentiation were determined through the deviation of the treated samples from the differentiation track. Analysis of three-day differentiation tracks revealed that the $24 \mathrm{~h}$ exposure time point gave the most significant deviation from the differentiation track after $\mathrm{MeHg}$ exposure, whereas $48 \mathrm{~h}$ and $72 \mathrm{~h}$ exposure samples did not deviate significantly from the track. This may be due to increasing sample variance over time, both in controls and treated samples, as well as to the fact that in the PCA plot the $24 \mathrm{~h}$ time point showed the highes dynamics in gene expression changes as compared to remaining time point (Figures 2D and 4ABC). Furthermore, the largest number of significantly regulated genes by $\mathrm{MeHg}$ exposure was observed at this time point, including many genes also regulated by time. Earlier studies with ESTc determined the same optimal time point for studying compound effects, and for similar reasons [83]. In the classic ESTc, MeHg developmental toxicity was misclassified using the classical prediction model of the ECVAM validation study [175] and, depending on the predicting gene sets used, was borderline or not identified as a developmental toxicant in the same test system with transcriptomics analysis using the differentiation track [155]. In the present study we clearly showed a significant deviation of $\mathrm{MeHg}$ treated samples on the differentiation track after 24h exposure at a lower tested concentration, indicating that the ESTn is more sensitive for detecting $\mathrm{MeHg}$-induced developmental toxicity as compared to the ESTc. This is in line with the developmental toxic properties of $\mathrm{MeHg}$ which are primarily neurodevelopmental in nature $[144,172]$

In this study we described the process of neural differentiation in our ESC model at the whole-genome level. Using multiple types of analysis pluripotency was found to be reduced and neural development was induced with time. Furthermore, effects of $\mathrm{MeHg}$ on neural differentiation at the gene expression level could be identified using multiple methods, including the differentiation track. $\mathrm{MeHg}$ was shown to specifically enhance neural differentiation-related gene expression and reduce other embryonic differentiation routes, confirming other studies and contributing further molecular information accompanying MeHg embryotoxicity. Future studies will have to show whether this model can be applied more widely for neurodevelopmental toxicity testing of chemical compounds.

\section{topeseres}




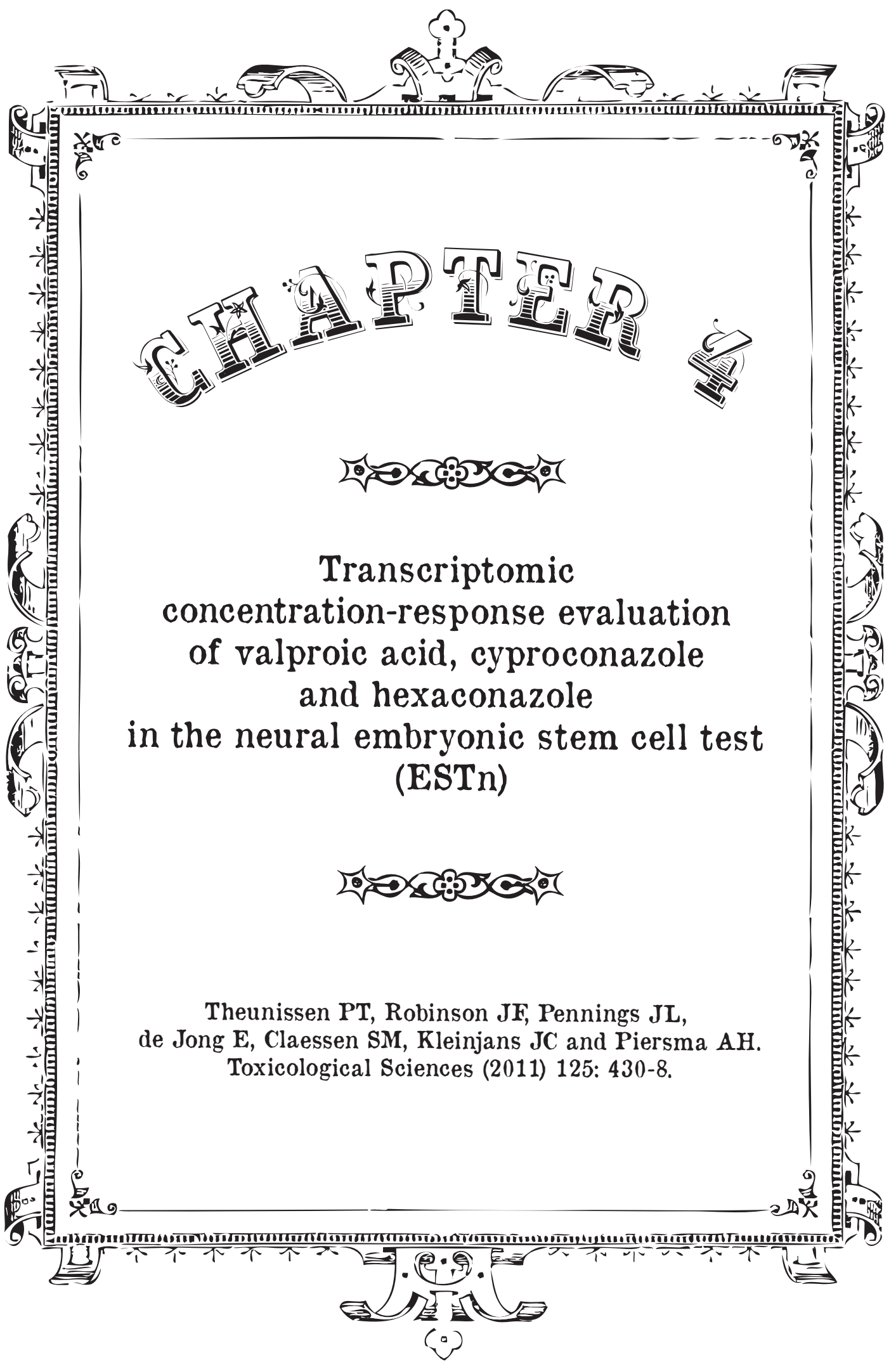




\section{Abstract}

A Lternative developmental toxicity assays are urgently needed to reduce A animal use in regulatory developmental toxicology. We previously designed an in vitro murine neural embryonic stem cell test (ESTn) as a model for neurodevelopmental toxicity testing [142]. Toxicogenomic approaches have been suggested for incorporation into the ESTn to further increase predictivity and to provide mechanistic insights. Therefore, in this study, using a transcriptomic approach, we investigated the concentration-dependent effects of three known (neuro)developmental toxicants, two triazoles, cyproconazole (CYP) and hexaconazole (HEX) and the anticonvulsant valproic acid (VPA). Compound effects on gene expression during neural differentiation and corresponding regulated gene ontology (GO) terms were identified after $24 \mathrm{~h}$ of exposure in relation to morphological changes on day 11 of culture. Concentration dependent responses on individual gene expression and on biological processes were determined for each compound, providing information on mechanism and concentration-response characteristics. All compounds caused enrichment of the embryonic development process. CYP and VPA but not HEX significantly enriched the neuron development process. Furthermore, specific responses for triazole compounds and VPA were observed within the GO-term sterol metabolic process. The incorporation of transcriptomics in the ESTn was shown to enable detection of effects which precede morphological changes and provides a more sensitive measure of concentration-dependent effects as compared to classical morphological assessments. Furthermore, mechanistic insight can be instrumental in the extrapolation of effects in the ESTn to human hazard assessment.

\section{\&8}

\section{Introduction}

UE to the high use of experimental animals needed for regulatory developmental toxicity testing $[10,12]$, there is increased momentum to develop, refine and establish in vitro screening assays to replace classical in vivo tests [34]. In the embryonic stem cell test (EST), mouse embryonic stem cells (mESC) are exposed to compounds during cardiomyocyte differentiation, giving an indication of developmental toxicity by scoring foci of beating cells [34]. The EST is widely being evaluated as a developmental toxicity screening assay in both chemical and pharmaceutical industry. The ECVAM (European Centre for Validation of Alternative Methods) validation study found this test to provide a predictivity score of $78 \%$, but subsequent validation studies were less successful $[37,176]$. To improve the predictivity of the EST, it was advised to add additional differentiation lineages to the testing strategy, such as neura and osteoblast differentiation and to use objective and mechanistic informative molecular techniques to determine compound effects [36, 37]. In this line of thought, we developed an 11-day mESC neural differentiation protocol (ESTn). Using this assay, we were able to detect specific adverse effects of the neurodevelopmental toxicant methylmercury ( $\mathrm{MeHg}$ ) [142], a compound which was misclassified in the ECVAM EST validation study [17]. Further investigation using additional developmental toxicants are needed to establish the applicability domain of this test for developmental toxicity testing.

Toxicogenomic approaches might be incorporated into alternative test systems to further increase predictivity and provide mechanistic insight. In our initial transcriptomic study in the ESTn, we characterized gene expression changes over time in non-exposed cultures and described the specific time-dependent gene expression profile after $\mathrm{MeHg}$ exposure [177]. Recent transcriptomic studies in the cardiac EST (ESTc) have shown that testing a compound at one concentration provides only limited information on the effect profile of the compound $[83,155]$. A recent study describing the effects of flusilazole in the ESTc illustrated the added value of concentration-response testing in EST transcriptomics experiments [81].

Therefore, in this study, a similar concentration-response approach was performed, to discern effects of three model compounds on neural differentiation in the ESTn: the triazole fungicides cyproconazole (CYP) and hexaconazole (HEX) and the anticonvulsant, valproic acid (VPA). CYP and HEX have been identified to be developmentally toxic in vivo [178-180]. In rats, CYP increased the prevalence of skeletal malformations, including delayed ossification of 
skull bones, cleft palate [178], hydrocephalus and hydronephrosis [180]. In the cardiac differentiation EST, CYP has been shown to induce neural differentiation [15], inducing deviation from the normal differentiating pattern. In rats, HEX caused skeletal malformations, however, no specific effects on neura development have been observed in vivo or in vitro $[15,179]$. The widely used anticonvulsant and histone deacetylase (HDAC) inhibitor VPA is well known for its induction of central nervous system (CNS) congenital abnormalities, including spina bifida and other neural tube defects (NTDs), but also heart anomalies and skeletal malformations [181, 182]

Using these three model compounds (CYP, HEX, VPA), we investigated the concentration-dependent effects of compound exposure in the ESTn on gene expression in relation to neural differentiation in vitro and to morphologica effects observed in vivo. Using a toxicogenomic approach, we are able to discriminate between compound classes and between mechanisms of action on the basis of differential gene expression during neural differentiation.

\section{ఠి}

\section{Materials and Methods}

\section{Embryonic Stem Cell Culture and Neural Differentiation}

Murine embryonic stem cells (ESC) (ES-D3, ATCC, Rockville, MD, USA) were routinely sub-cultured every $2-3$ days and grown as a monolayer in Dulbecco's Modified Eagle Medium (DMEM)-based (Gibco BRL, Gaithersburg, MD, USA) medium supplemented with leukemia inhibiting factor (LIF) (Chemicon, Temecula, CA, USA) at a final concentration of 1000 units $/ \mathrm{ml}$. The cells were maintained in a humidified atmosphere at $37^{\circ} \mathrm{C}$ and $5 \% \mathrm{CO}_{2}$. Induction of neural differentiation was performed as described earlier [142, $183]$.

\section{Resazurin cell viability assay}

To determine compound effects on cell viability, a Resazurin dye reduction assay (Promega, Leiden, The Netherlands) was used as a measure for the number of viable cells, as described previously [142]. Cyproconazole (CYP) (CAS number 94361-06-5, Sigma-Aldrich, Zwijndrecht, The Netherlands), hexaconazole (HEX) (CAS number 79983-71-4, Sigma-Aldrich, Zwijndrecht, The Netherlands) and valproic acid sodium salt (VPA) (CAS number 1069-66-5, Sigma-Aldrich, Zwijndrecht, The Netherlands) were diluted in dimethy sulfoxide (DMSO), and cell cultures (including controls) were exposed to a final DMSO concentration of $0.1 \%$, which did not affect cell viability. Viability was tested in the following concentration ranges: CYP $0.1-300 \mu \mathrm{M}$, HEX $0.01-300 \mu \mathrm{M}$ and VPA $0.1-2.5 \mathrm{mM}$. Tests were performed in triplicate.

\section{Treatment and morphological scoring for effects on neurite} outgrowth

Highest concentrations tested were selected on basis of the Resazurin viability assay, wherein the highest concentration provided a minimum viability of $80 \%$, or $1000 \mu \mathrm{M}$, chosen as the highest concentration feasible in vivo. A concentration of $100 \mu \mathrm{M} \mathrm{CYP}$ and $25 \mu \mathrm{M}$ HEX reduced viability to $80 \%$ and $1000 \mu \mathrm{M}$ VPA did not affect cell viability in the ESTn. For both morphologica and gene expression assessments, compounds were tested at the following concentrations: CYP $1.5 \mu \mathrm{M}, 6.0 \mu \mathrm{M}, 25 \mu \mathrm{M}$ and $100 \mu \mathrm{M}$; HEX $0.5 \mu \mathrm{M}, 1.5$ $\mu \mathrm{M}, 6.0 \mu \mathrm{M}$ and $25 \mu \mathrm{M}$; VPA $15 \mu \mathrm{M}, 60 \mu \mathrm{M}, 250 \mu \mathrm{M}$ and $1000 \mu \mathrm{M}$. Contro embryoid bodies (EB) were treated with $0.1 \%$ DMSO. For morphological scoring, cultures were treated for $72 \mathrm{~h}$ from initiating suspension culture on day 3 of the protocol until the start of the serum free period on day 6 (Theunissen et al., 2011). Morphological effects were determined by assessment of the extent of neurite outgrowth from the EB, at the end of the differentiation protocol (day 11), observed using an IX51 inverted microscope (Olympus, Zoeterwoude, The Netherlands) with CellD software (Olympus, Zoeterwoude, The Netherlands). Morphology of neurite outgrowth was scored as the percentage of neural corona surrounding each $\mathrm{EB}$, irrespective of the distance of outgrowth from the EB. For scoring neurite outgrowth, a cutoff was chosen at or $\geq 75 \%$ neurite outgrowth around the $\mathrm{EB}$, based on historical control data. For each concentration, three cultures each with 30 to $40 \mathrm{~EB}$ were assessed. Parallel cultures were harvested for gene expression analysis at earlier time points.

\section{RNA isolation and whole-genome expression profiling}

Exposed differentiation cultures were treated with concentrations as described earlier from day 3 of the protocol onwards and sampled after $24 \mathrm{~h}$ exposure day 4) (8 replicates per group). The $24 \mathrm{~h}$ exposure was chosen as the time poin expressing the least variability between samples as observed in our earlie time-course study with the neurodevelopmental toxicant MeHg [183]. RNA from control differentiation cultures was sampled on protocol days 3 ( 8 replicates), 4 (5 replicates), and 5 (5 replicates). Gene expression background over time correlated well with previous studies [183]. Cells (approximately 30-40 EB/sample) were directly collected in RNA Protect (Qiagen Benelux, Venlo, The Netherlands) to stabilize RNA, and total RNA was purified using the Qiagen RNeasy Plus Mini Kit (Qiagen Benelux, Venlo, The Netherlands 
following manufacturer's instructions. RNA quantity was determined using the NanoDrop Spectrophotometer (Isogen Lifescience, de Meern, The Netherlands). RNA integrity was assessed on the 2100 Bioanalyzer (Agilent, Amstelveen, The Netherlands) using the RNA 6000 Nano Chip Kit (Agilent, Amstelveen, The Netherlands), and good quality RNA was used for gene expression analysis (RNA integrity number (RIN) > 8.0). Gene expression analysis using the Mouse Genome 4302.0 arrays (Affymetrix, Santa Clara, CA, USA) was performed as described previously [183]. Quality controls, including scaling factors, average intensities, present calls, background intensities, noise, and raw $\mathrm{Q}$ values, were performed according to the manufacturer's instructions and showed to be within acceptable limits for all chips.

\section{Data Analysis and Statistics}

Affymetrix CEL files were normalized using the Robust Multichip Average (RMA) algorithm [158] using RMAexpress [159]. For probe to gene mapping, a custom Chip Description File (CDF) was used according to the assembly by de Leeuw et al. [160] (http://mad-db.science.uva.nl/ wdeleeuw/HybridAnnot/ version6.html). Of the hybrid probe-set definitions included in the custom annotation, 16,331 probe sets defined by the Brainarray custom CDF version 11 (http://brainarray.mbni.med umich.edu/Brainarray/Database/CustomCDF) [161] and 4648 additional probe sets defined by Affymetrix chip annotation 26 were used in further analyses, giving a total of 20,979 probe sets. Probe sets for Affymetrix internal controls or probe sets that did not correspond to an Entrez Gene ID were not used in further analyses. Statistical analyses were carried out using the R statistical software environment (http://www.R-project. org) using log-transformed values. For each gene, maximal fold change (FC) in gene expression between the experimental groups were determined by comparing the average normalized signal values per group and were calculated as the maximum/minimum ratio. For compound toxicity effects, the treated samples were compared to the time-matched day 4 controls. Genes differentially expressed between any of the experimental groups (four concentrations and control) were identified by a one-way ANOVA. A significance threshold of FDR $<0.01$ were used to select genes that were significantly differentially expressed due to compound exposure. Functional annotation and enrichmen for Gene Ontology (GO) biological processes were studied using DAVID (http://david.abcc.ncifcrf.gov/) [162] and GenMAPP [96]. Significantly enriched GO categories were based on a set criteria of permutation value $(\mathrm{p}<0.01), Z$-score $(>2)$, and genes changed within each specific GO gene category $(>5)$. GO-terms with $>5000$ genes and redundant GO-terms were not included in the analysis. To quantitatively evaluate changes within gene ex- pression-linked GO gene categories, we used GO-Quant [95] to calculate the average absolute $\log 2$ fold change (FC) associated with genes within each GO subset for each compound separately. Venn diagrams describing relations of significant gene expression and enrichment of GO-terms between compounds, were created using Venny (Oliveros, 2007). Hierarchical clustering of changes to control for all significantly differentially expressed genes (or subsets thereo selected on the basis of venn diagrams and GO-terms) was performed in GeneMaths XT (Applied Maths, Sint-Martens-Latem, Belgium), using Euclidean distance and Ward linkage.

\section{@̊?}

\section{Results}

Concentration-dependent effects of CYP, HEX and VPA on viability and neurite outgrowth

CYP, HEX and VPA affected cell viability in a concentration-dependent manner (Figure 1). Compound concentrations reducing cell viability to $80 \%$ (IC20) were 117.6 $\mu \mathrm{M}$ for CYP, $19.8 \mu \mathrm{M}$ for HEX and $2200 \mu \mathrm{M}$ for VPA. This data was used as a reference level for the highest concentration tested in the ESTn for both gene expression and neural morphological assessments. On day 11, after a $72 \mathrm{~h}$ compound exposure from day 3 onwards, both CYP and VPA significantly altered neurite outgrowth in the ESTn in a dose responsive manner (Figure 1). At the tested concentrations $(0-25 \mu \mathrm{M})$, HEX did not significantly impact neurite outgrowth at concentrations tested. However, in earlier performed concentration range-finding experiments, HEX did reduce neurite outgrowth at $100 \mu \mathrm{M}$ (data not shown), a highly cytotoxic concentration at which viability was reduced to approximately $10 \%$.

\section{Gene expression is regulated by CYP, HEX and VPA in a \\ concentration-dependent manner}

Compound-induced differential gene expression was compared between exposed murine ESC neural differentiation cultures and time-matched contro cultures, using one-way ANOVA $(\mathrm{FDR}<0.01)$. We identified 663, 761 and 3626 genes to be significantly regulated by CYP, HEX and VPA, respectively (Figure 2a). CYP regulated 0, 215, 134, 281 genes with increasing concentrations tested. For Hex and VPA, these numbers were 0, 20, 87, 779 and 0, 147, 574 2355 , respectively. We observed 252 genes to be significantly regulated by all three compounds. In total, the expression levels of 3980 unique genes were concentration-dependently regulated by any of the compounds (Figure 2b). 
A

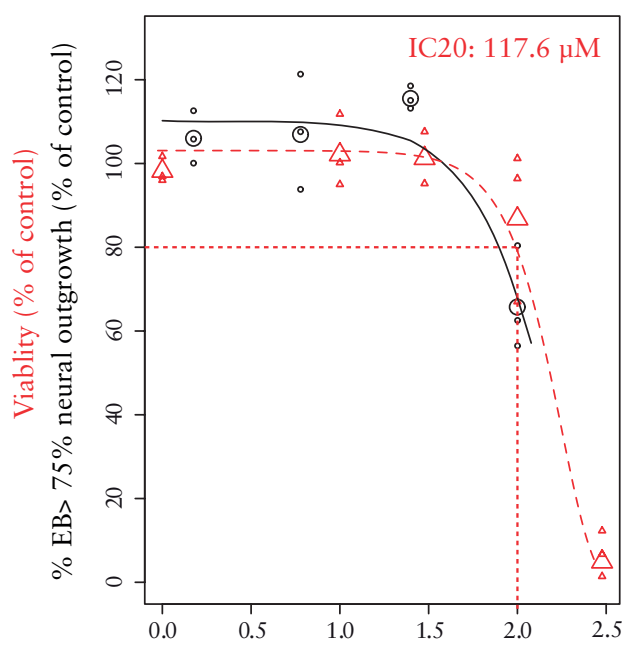

$\log 10-$ Concentration Cyproconazole $(\mu \mathrm{M})$

B

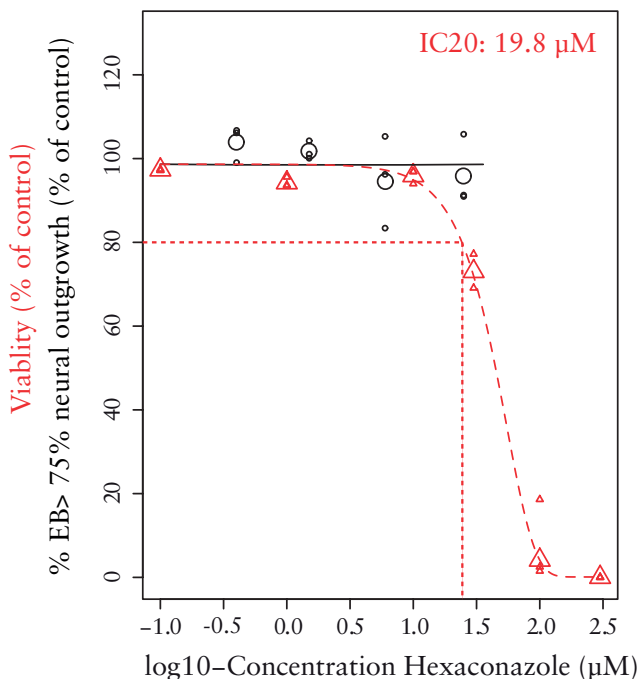

Figure 1 CYP, HEX and VPA effects on viability and morphological neuron out growth. Concentration response effects on cell viability in ESC (CYP $0.1-300 \mu \mathrm{M}$, HEX $0.01-300 \mu \mathrm{M}$ and VPA $0.1-2.5 \mathrm{mM}$ ) (triangles) and concentration response effects on the percentage of EB with $>75 \%$ neurite outgrowth (CYP $1.5 \mu \mathrm{M}, 6.0 \mu \mathrm{M}$, $25 \mu \mathrm{M}$ and $100 \mu \mathrm{M}$; HEX $0.5 \mu \mathrm{M}, 1.5 \mu \mathrm{M}, 6.0 \mu \mathrm{M}$ and $25 \mu \mathrm{M}$; VPA $15 \mu \mathrm{M}, 60 \mu \mathrm{M}$, $250 \mu \mathrm{M}$ and $1000 \mu \mathrm{M}$ ) (circles) for A) CYP (IC20: $117.6 \mu \mathrm{M}$ ), B) HEX (IC20: 19,8 $\mu \mathrm{M})$ and C) VPA (IC20: $2200 \mu \mathrm{M})$.

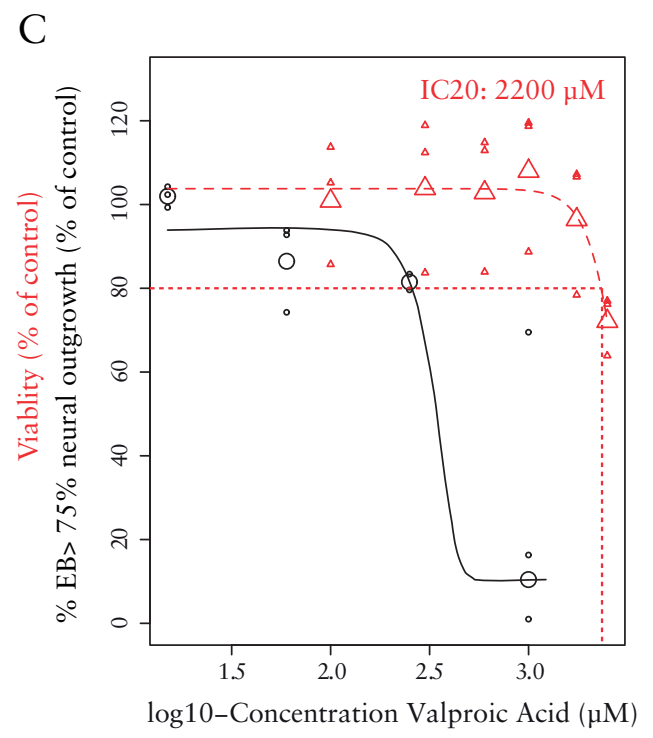

Figure 1 Continued

These genes were significantly differentially expressed after exposure to at least one of the compounds in at least one test compound concentration, as determined by one way ANOVA including all tested concentrations plus control.

\section{GO-terms regulated by CYP, HEX and VPA specifically}

To study the effects of compounds at the biological process level, GO-terms significantly enriched by each of the three compounds were identified (Figure 2c). Within the 663 genes observed to be significantly altered by CYP, 93 enriched GO-terms were identified (Figure 2c), including GO-terms involved in general development (e.g. general, embryonic, central nervous system, organ), differentiation (cell, neuron), metabolism (sterol, lipid, isoprenoid, organic) transcription, biological regulation, cell cycle and protein kinase pathways (data in supplementary data of original article). For the 761 genes regulated by HEX, only $28 \mathrm{GO}$ biological processes were enriched (Figure 2c), including GO-terms related to development (e.g. general, embryonic, CNS, organ), metabolism (lipid, sterol, isoprenoid, organic), cell cycle and transcription (data in supplementary data of original article). Within the 3626 genes regulated by VPA, 74 distinct GO-terms were enriched (Figure 2b), related to a wide array of processes, including development (general, embryonic, central 
nervous system, organ), differentiation (cell, neuron), metabolism (sterol, lipid, isoprenoid, organic, nucleic acid and protein), cell cycle, cell death, oxidative stress and localization (data in supplementary data of original article) Significance $(\mathrm{p} \leq 0.01)$ and degree of significance (indexed as $\mathrm{z}^{*}-\log (\mathrm{p}$-value) for selected GO-terms is shown in figure $2 \mathrm{~d}$ (complete list of GO-term significance values is provided in the in supplementary data of the original article), to illustrate degrees of GO-term enrichment. For example steroid metabolic process is highly enriched in the triazoles compounds, but only slightly enriched in VPA, whereas neuron development is highly enriched in VPA, but slightly enriched in CYP and not enriched in HEX.

The 12 GO-terms significantly enriched by all three compounds were related to embryonic morphogenesis, metabolic processes (including sterol and lipid metabolic process) and regulation of cell cycle (Figure 2C). The 26 GO-terms regulated by CYP and VPA but not HEX were involved in general development (e.g. developmental process, anatomical system development), nervous system development (neuron development, axon guidance) and cell differentiation and morphology (Figure 2C). The 10 GO-terms significantly enriched by CYP and HEX but not VPA were involved in steroid metabolic processes, embryonic development and RNA processing (Figure $2 \mathrm{C}$ ). No GO-terms were significantly enriched by both HEX and VPA which were not also enriched by CYP.

Concentration-dependent effects of genes within selected enriched GO-term (embryonic morphogenesis, neuron development, regulation of cell cycle, programmed cell death, sterol metabolic process and RNA processing) were examined for CYP, HEX and VPA (Figure 3ABC). Concentration dependent changes in gene expression were associated with selected GO-terms. For example, 21 genes associated with sterol metabolic process on average showed a concentration dependent increase in effect with CYP with a peak change in the high dose of 1.5 FC. Across selected functional groups, we observed genes associated with embryonic morphogenesis followed by neuron differentiation to have greater changes as compared to genes associated with regulation of cell cycle and programmed cell death with all three compounds at all concentrations tested.

Effects on expression changes of single genes within the GO biological processes embryonic morphogenesis (Figure 4A), neuron development (Figure $4 \mathrm{~B}$ ) and sterol metabolic process (Figure 4C) were visualized by hierarchical clustering. Genes significantly regulated $(\mathrm{FDR}<0.01)$ by at least two out of three compounds are shown. In general, independent of significance, genes
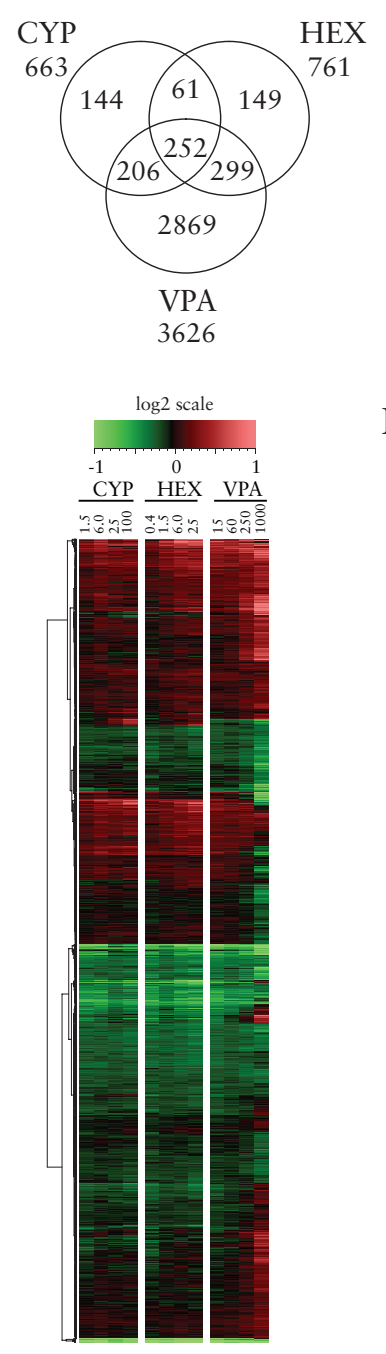
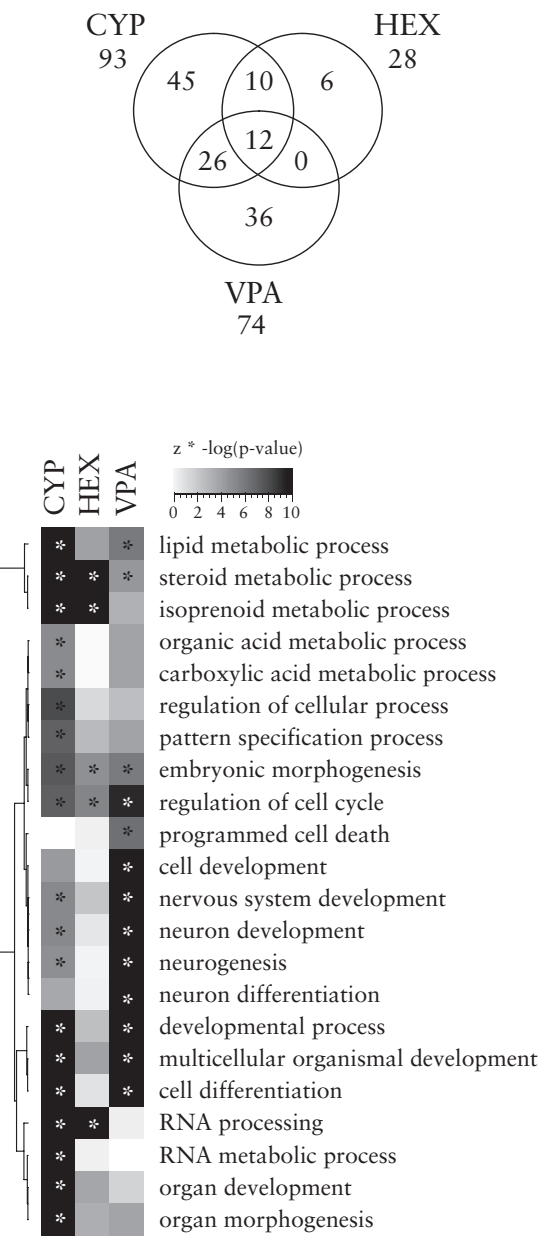

Figure 2 Venn diagrams and hierarchal clustering plots. Venn diagrams and (ierarchal clustering plots describing A) overlap of genes significantly concentrationdependently expressed for CYP (663 genes), HEX (761 genes) and VPA (3625 genes) (FDR 0.01) and B) Hierarchal clustering plot of all 3980 genes regulated by any of the compounds (One-way ANOVA FDR $<0.01, \log 2$ scale). C) overlap of GO-terms concentration-dependently enriched for CYP (93), HEX (28) and VPA (74) ( $p<0.01, \mathrm{z}>2$, number changed $>5$ ). D) Hierarchal clustering plot of GO biological processes showing significance of concentration-dependent enrichment response by CYP, HEX or VPA ** $=\mathrm{p} \leq 0.01$, grey scale $=\mathrm{z}$-score $*-\log (\mathrm{p}$-value $))$. 
A

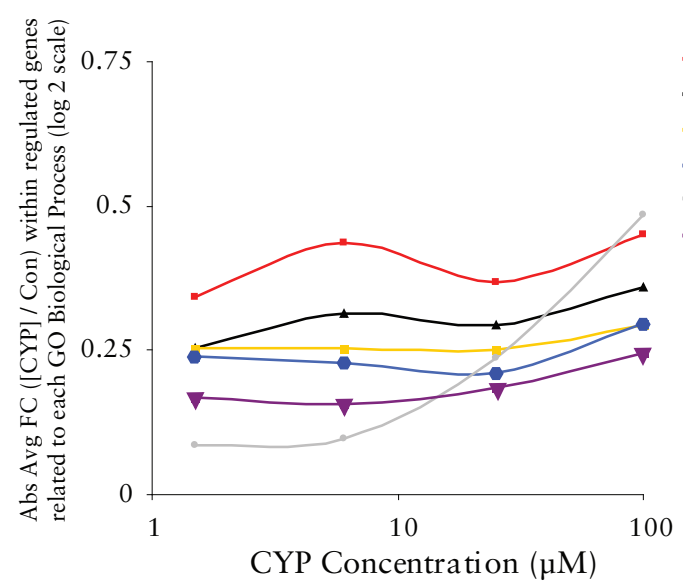

$\mathrm{B}$

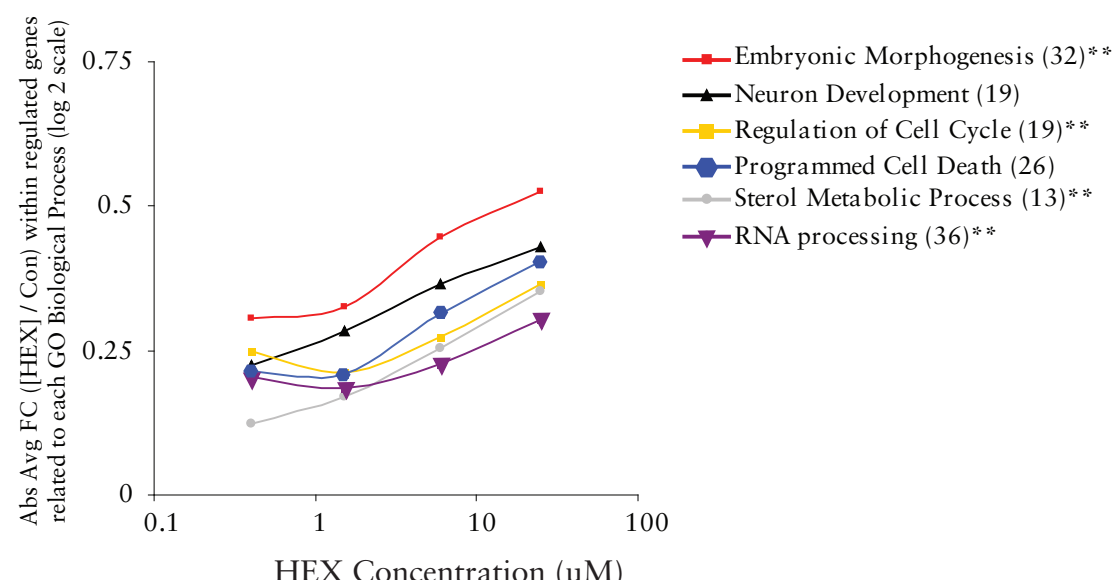

Figure 3 Compound effects on GO-terms fold change. Average absolute FC of selected key biological processes (embryonic morphogenesis, neuron development, regulation of cell cycle, programmed cell death, sterol biosynthetic process and RNA processing) for A) CYP, B) HEX and C) VPA. (** = significantly enriched GO-term by compound)

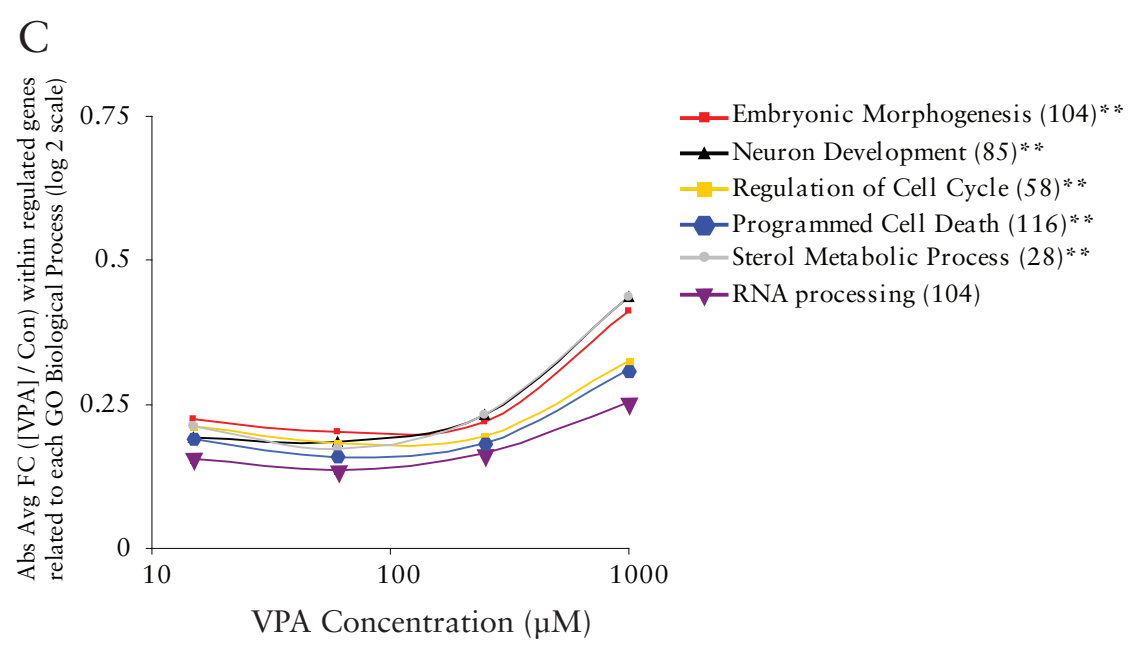

Figure 3 Continued

associated with embryonic morphogenesis were regulated by all three compounds in the same concentration responsive manner in terms of directionality, with the exception of the highest concentration of VPA tested $(1000 \mu \mathrm{M})$. For approximately $50 \%$ of embryonic morphogenesis genes regulated by two of the three compounds, VPA at $1000 \mu \mathrm{M}$ resulted in differential directional regulated expression compared to the other exposure groups (Figure 4A). Within the GO-term neuron development (Figure 4B), a similar pattern of concentration-dependent regulation was observed across the three compounds, again with VPA at $1000 \mu \mathrm{M}$ regulating genes in an opposite direction compared to lower doses and to the triazole compounds. Within the GO-term sterol metabolic process (Figure 4C), the majority of the genes were concentration-dependently upregulated in the triazole compounds, but concentration-dependently downregulated in VPA. For example, Cyp51, a gene which is important in sterol metabolism, but also in retinoic acid (RA) metabolism, was upregulated in a concentration-dependent way by the triazole compounds, but downregulated by VPA. 
A
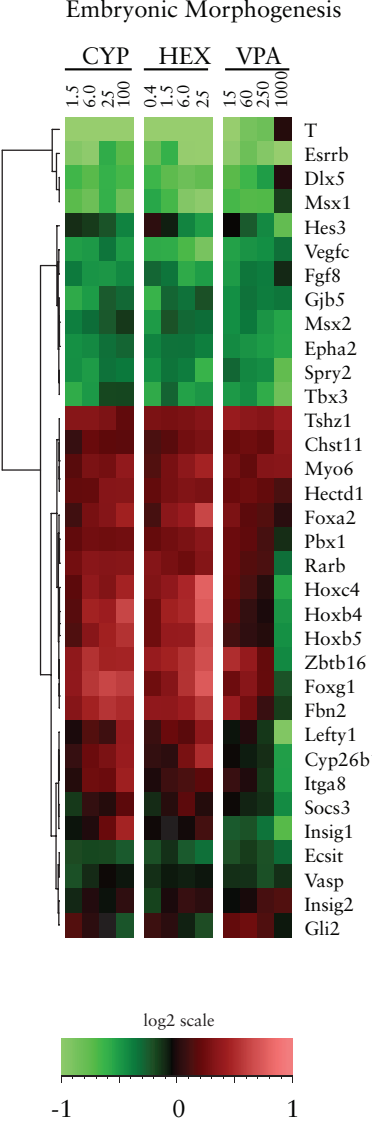

B
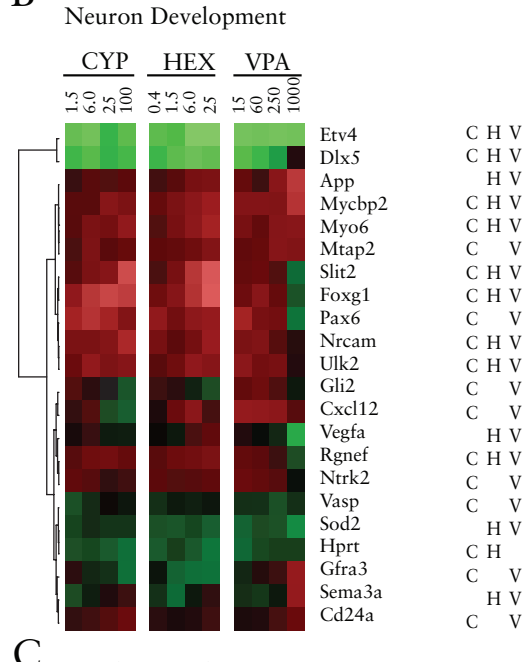

C$$
\text { CYP HEX VPA }
$$

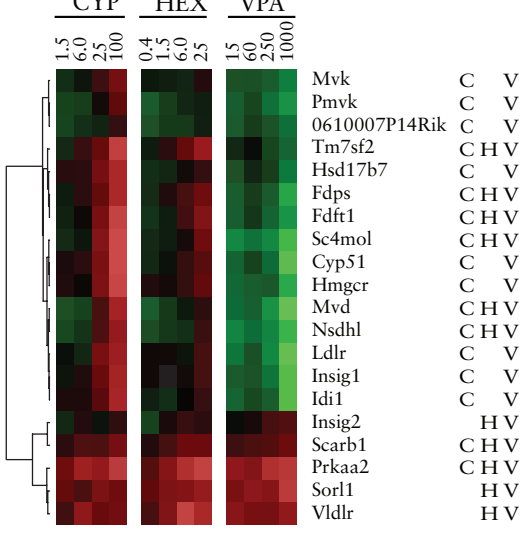

Figure 4 Genes significantly concentration-dependently expressed in at least two of three compounds CYP $(\mathrm{C}), \operatorname{HEX}(\mathrm{H})$ and VPA $(\mathrm{V})$ compared to control $(\mathrm{FDR}<0.01$, $\log 2$ scale) for the GO-terms A) Embryonic Morphogenesis, B) Neuron Development and C) Sterol Metabolic Process

\section{Discussion}

I recent years, several additions to the EST developmental toxicity assay have been proposed, including the introduction of additional differentiation routes $[36,37]$. We developed the complementary ESTn model to predict compound effects on the neuroectodermal lineage and investigated the effects of the neurodevelopmental toxicant $\mathrm{MeHg}$ [142]. Studies describing molecular endpoints [184-186] and transcriptomics techniques [40, 83, 187] in the EST proved to be useful in further increasing the predictivity of the EST $[84,155]$. Therefore, we studied the effects of MeHg over time in the ESTn using transcriptomics [177] and concluded that the most robust measurements can be achieved after $24 \mathrm{~h}$ treatment on day 4 of the standardized differentiation protocol. In the present study, concentration-dependent transcriptomic responses are studied testing three known developmental toxicants cyproconazole (CYP), hexaconazole (HEX) and valproic acid (VPA) to further characterize the ESTn. The compounds were chosen such that we should be able to discriminate a triazole specific response (CYP and HEX versus VPA) and a neurodevelopmental toxicity specific response (CYP and VPA versus HEX), thus investigating whether the differential gene expression responses would display mechanistic specificity and add greater value than morphological assessments alone.

\section{Relationships between compound effects on morphology and} gene expression changes

CYP, HEX and VPA impacted cell viability with different potencies, with HEX being the most potent and VPA the least. Compound effects on neurite outgrowth around the EB were concentration-dependent for CYP and VPA VPA severely reduced neurite outgrowth at concentrations without reduced viability, with approximately a 10 fold difference between the IC20 for viability and the ID20 for differentiation, showing that neurite outgrowth is a much more sensitive parameter compared to viability in this assay specifically for VPA. In contrast, only minor sensitivity differences were observed for CYP between compound effects on cytotoxicity and neurite outgrowth. We did not observe any sensitivity differences between cytotoxicity and neurite outgrowth for HEX. Associated with effects on cell viability and neurite outgrowth, we observed concentration-dependent effects on gene expression and corresponding functional GO-terms. Despite using a similar range in potencies, VPA significantly altered the expression of approximately four times more genes than CYP and HEX, primarily driven by the greater gene expression response at the highest concentration tested, which was in line with the relatively large effect on neurite outgrowth in the morphological test. 


\section{Concentration-dependent compound effects on GO-term} enrichment

Analysis of GO-terms regulated revealed major themes within genes significantly enriched by CYP, HEX and VPA. In association with concentration-dependen effects on morphology, we observed common enrichment of GO-terms related to development, metabolism and cell cycle. Uniquely, GO-terms related to cell differentiation and neuron differentiation were only significantly enriched by CYP and VPA, and not by HEX. In addition, published data have also shown neurodevelopmental effects for CYP and VPA but not HEX, confirming the correlation between ESTn gene expression readout and existing in vivo and in vitro data [15, 179-181]. VPA specifically regulated programmed cell death and apoptosis related GO-terms, at a concentration that was not found to be cytotoxic in culture. VPA, which is also an HDAC inhibitor, is known to induce apoptosis through this mechanism [188]. Enrichment of apoptosis accompanied with no observed cytotoxicity in the cells was also observed earlier in the pluripotent mouse embryonal carcinoma cell line after $24 \mathrm{~h}$ exposure to $1 \mathrm{mM}$ VPA [76]. In addition, effects on development related GO-terms were observed at concentrations at which no effects were observed on neural morphology or cell viability, implicating that gene expression can be a more sensitive endpoint.

\section{CYP, HEX and VPA disturb genes involved in embryonic} development

Most GO-terms were already regulated at the lowest concentration and concentration-dependently upregulated in all three compounds, indicating that effects on the RNA transcript level can occur without inducing adverse effects on morphology. The direction of gene regulation within embryonic morphogenesis was comparable for the triazoles, signifying a comparable mode of action on the regulation of this GO-term. However, VPA regulated gene expression of well characterized developmental associated genes in an opposite direction compared to the triazoles (Figure 4a), of which the majority is also important during neural development. These included Hox-pathway related genes (Hoxb4, Hoxc4, Hoxb5, Dlx5, Msx1) [189], genes of the TGFpathway (Lefty1) [190], early ectodermal formation and patterning (Fgf8) [191] early mesodermal formation (T) [192] and retinoic acid pathway related genes (Rarb, Cyp26b1) [193, 194]. The different direction in which these genes are regulated by VPA as compared to the triazoles, suggests a different mechanism of action, leading to comparable morphological outcomes in the ESTn.
CYP and VPA responses significantly target neural development Genes regulated within the neuron development GO-term showed a simila response for both the triazole compounds and VPA at the gene level, although only CYP and VPA significantly enriched this GO-term. Genes regulated by both CYP and VPA included Mtap2 which is important for dendrite microtubule formation during neuron development [141] and the well characterized Pax6, an important transcription factor during early ectodermal development [195]. Compound effects on the genes within the neuron development GO-term observed in the ESTn corresponded with developmental toxic effects found in vivo. VPA is well known for its induction of spina bifida and NTDs [181] and CYP was observed to increased incidence of hydrocephalus in rats [180] and furthermore induced neural differentiation in the ESTc [15]. No effects of HEX on neural development have been observed in vivo [15, 179], which is in line with the absence of a significant regulation of the neuron development GO-term in this study.

CYP and HEX increase sterol metabolic process related gene expression, whereas VPA decreases their expression

As a main mechanism of action, triazoles inhibit the enzyme lanosterol 14- $\alpha$ demethylase (Cyp51) which catalyses the synthesis of ergosterol, an important protein for membrane integrity [196]. Valproic acid was observed to inhibit sterol synthesis in the developing rat brain [197] and more recent studies show that VPA causes a general down-regulation of expression of genes encoding for enzymes early in steroidogenesis in the human carcinoma cell line H295R and mouse Y1 adrenocortical cells [198, 199]. All three compounds significantly regulated pathways involved in sterol synthesis in a concentration-dependent manner. For example, in the GO-term sterol metabolic process, gene expression was increased by both triazoles, however, VPA showed a decrease in the majority of the genes regulated in this pathway. Cyp51, for instance, was upregulated by CYP but downregulated by VPA. This indicates that, although all three compounds have an effect on sterol metabolism, the mechanisms behind the effect are clearly different.

HDAC inhibition pathways are specifically disturbed by VPA A well characterized mode of action of VPA is HDAC inhibition [200], a mechanism by which VPA is thought to change overall gene expression [188, 201, 202]. A core set of 15 gene markers regulated by three HDAC inhibitors was described earlier [76, 200]. Out of these 15 genes, 9 genes were significantly regulated $(\mathrm{FDR}<0.01)$ by VPA in this study, whereas the triazoles CYP and HEX only regulated 2 of these genes (data not shown). Furthermore, direction 
of regulation by VPA was comparable to effects observed earlier in the pluripotent mouse embryonal carcinoma cell line P19 [76], showing that this VPA specific main mechanism of action could be discerned in the ESTn.

\section{Conclusion}

The present study showed that differential gene expression provides a sensitive tool for studying the effects of chemicals on neural differentiation in the EST detecting effects at concentrations below those inducing morphological effects. Moreover, chemical-specific gene expression signatures can be discriminated such as the triazole signature and the neurodevelopmental toxicity signature in the present study, which allow mechanistic insight into the action of chemicals tested. This methodology therefore potentially offers significant added value to existing morphological effect assessment in ESTn and to the elucidation of the nature of developmental neurotoxicity of chemicals. Such mechanistic insights are instrumental in determining the extrapolation of adverse effects in alternative assays to human hazard assessment.

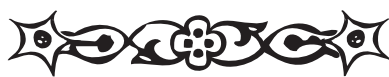




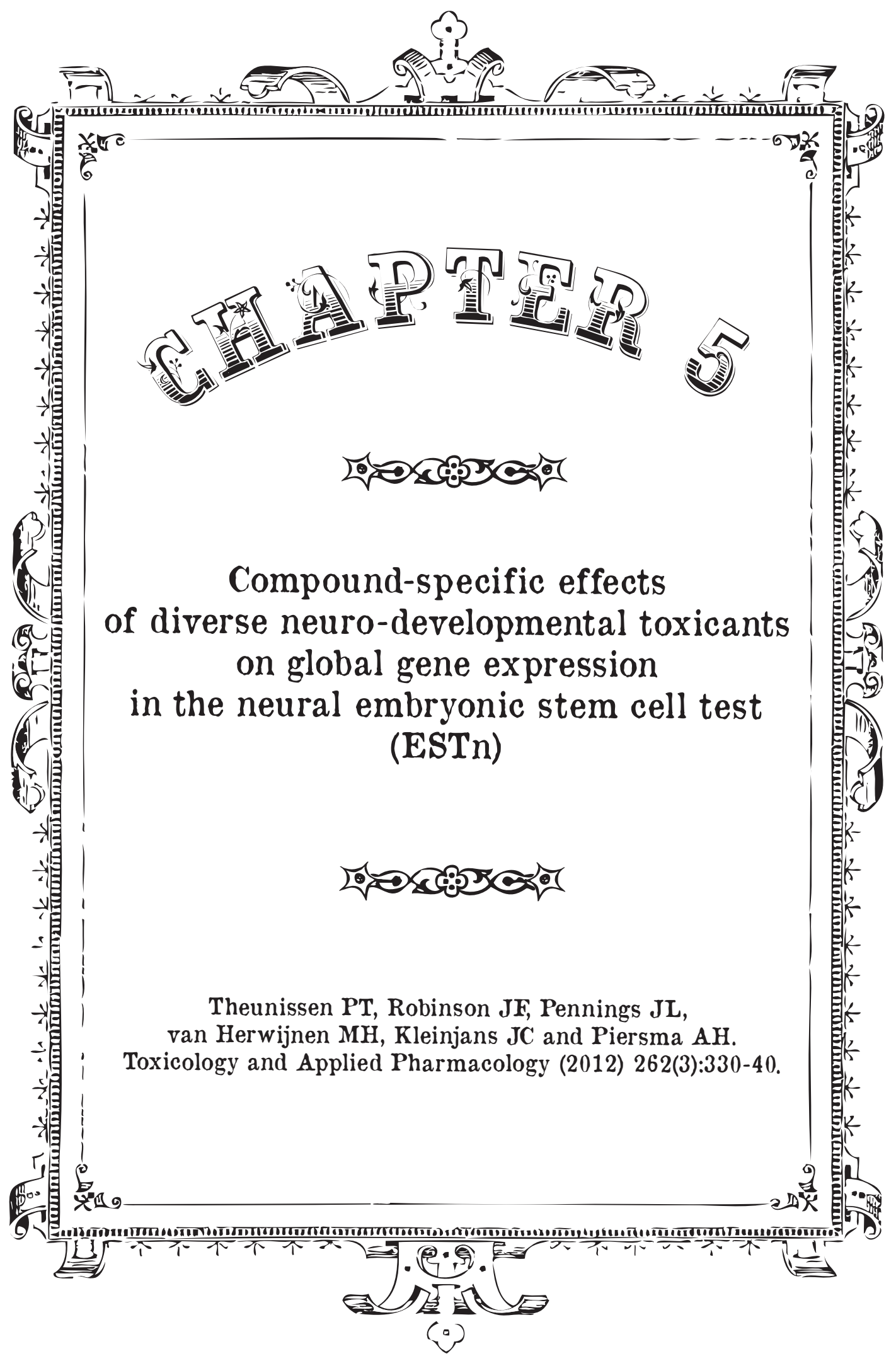




\section{Abstract}

A Lternative assays for developmental toxicity testing are needed to reduce A animal use in regulatory toxicology. The in vitro murine neural embryonic stem cell test (ESTn) was designed as an alternative for neurodevelopmental toxicity testing. The integration of toxicogenomic-based approaches may further increase predictivity as well as provide insight into underlying mechanisms of developmental toxicity. In the present study, we investigated concentrationdependent effects of six mechanistically diverse compounds, acetaldehyde (ACE), carbamazepine (CBZ), flusilazole (FLU), monoethylhexyl phthalate (MEHP), penicillin $\mathrm{G}$ (PENG) and phenytoin (PHE), on the transcriptome and neural differentiation in the ESTn. All compounds with the exception of PENG altered ESTn morphology (cytotoxicity and neural differentiation) in a concentrationdependent manner. Compound induced gene expression changes and corresponding enriched gene ontology biological processes (GOBP) were identified after $24 \mathrm{~h}$ exposure at equipotent differentiation-inhibiting concentrations of the compounds. Both compound-specific and common gene expression changes were observed between subsets of tested compounds, in terms of significance, magnitude of regulation and functionality. For example, ACE, CBZ and FLU induced robust changes in number of significantly altered genes ( $\geq 687$ genes) as well as a variety of GOBP, as compared to MEHP, PHE and PENG ( $\leq 55$ genes with no significant changes in GOBP observed). Genes associated with developmentally related processes (embryonic morphogenesis, neuron differentiation and Wnt signaling) showed diverse regulation after exposure to ACE, CBZ and FLU. In addition, gene expression and GOBP enrichment showed concentration dependence, allowing discrimination of non-toxic versus toxic concentrations on the basis of transcriptomics. This information may be used to define adaptive versus toxic responses at the transcriptome level.

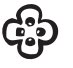

\section{Introduction}

URRENT legislation for chemical and pharmaceutical developmental toxicity testing requires a high number of experimental animals, creating momentum for development of alternative screening assays to precede or replace classical in vivo tests $[10,12]$. The embryonic stem cell test (EST) was designed as a screening assay for developmental toxicity of compounds [18]. The EST is used to assess compound effects on murine embryonic stem cell (mESC) differentiation into cardiomyocytes, providing an indication of developmental toxicity by scoring foci of beating cells [34]. Initial validation studies using the cardiac EST (ESTc) $[17,37,176]$ suggest promise in predictivity of developmenta toxicants, but also areas of needed improvement, including refined protocol development, the implementation of additional differentiation routes (e.g. neural) and the introduction of objective and mechanistic informative techniques to determine compound effects [36, 37].

Previously, we described the mESC neural differentiation protocol (ESTn), in which we were able to detect compound-induced adverse effects on neura outgrowth using the model neurodevelopmental toxicant, methylmercury $(\mathrm{MeHg})[17,142]$. In order to provide further mechanistic insight and increase the predictivity of the ESTn, a transcriptomics approach was incorporated. We identified time-dependent induced gene expression alterations, providing a signature for $\mathrm{MeHg}$ developmental toxicity in the ESTn which preceded observed morphological effects in our system [177]. Furthermore, we were able to discriminate between compound classes and mechanisms of action on the basis of concentration dependent differential gene expression during neural differentiation for two triazole antifungal compounds and valproic acid [203]. In the present study, the added value of the transcriptomics readout in the ESTn is investigated and possible common biomarker gene sets describing (neuro)developmental toxicity are explored. We investigated concentrationdependent effects of six structurally and mechanistically diverse compound on the transcriptome in relation to effects on neural differentiation and viability (Table 1). Four out of six compounds investigated were selected based on thei in vivo induction of neurodevelopmental defects: the active ethanol metabolite acetaldehyde (ACE), anti-convulsants carbamazepine (CBZ) and phenytoin (PHE) and mono-ethylhexyl phthalate (MEHP), the active metabolite of bis(2ethylhexyl)phthalate (DEHP) [204-210]. Additionally, two in vivo non-neurodevelopmental toxic compounds were tested, of which flusilazole (FLU) was observed to induce skeletal malformations in vivo [211,212], and the antibiotic penicillin $\mathrm{G}$ (PENG), which is known to induce no developmental toxicity in mammals [213]. In order to select genes only differentially expressed at 
Table 1 Background information on compound developmental toxicity data

\begin{tabular}{|c|c|c|c|c|c|c|}
\hline Compound & Abbreviation & LEL (mg/kg BW) & Species & Developmental Toxicity & Proposed mode of action & Reference \\
\hline Acetaldehyde & ACE & Not reported & Human & Fetal alcohol syndrome (FAS) & $\begin{array}{l}\text { Disrupted cell-cell interactions, oxidative stress, growth factor } \\
\text { signaling disruption, altered gene expression }\end{array}$ & [206] \\
\hline \multirow[t]{2}{*}{ Carbamazepine } & CBZ & $>1000$ & Rat & None & Unknown & \\
\hline & & Not reported & Human & $\begin{array}{l}\text { Small head circumference, } \\
\text { mild facial, dysmorphia, } \\
\text { and developmental delay }\end{array}$ & & [205] \\
\hline \multirow[t]{2}{*}{ Flusilazole } & FLU & 0,4 & Rat & Urogenital malformations & $\begin{array}{l}\text { Disturbance of CYP26 metabolic pathway [224], disturbance of } \\
\text { sterol biosynthesis through Cyp450 }\end{array}$ & [212] \\
\hline & & 20 & Mice & Skeletal malformations & & [211] \\
\hline \multirow[t]{2}{*}{ MEHP } & МЕHР & 0,5 & Mouse & $\begin{array}{l}\text { Anterior neural tube defects } \\
\text { and skeletal abnormalities }\end{array}$ & Endocrine disruption & {$[207,208]$} \\
\hline & & 100 & Rat & $\begin{array}{l}\text { Neural tube defects and } \\
\text { vascular abnormalities }\end{array}$ & & [209] \\
\hline Penicillin G & PENG & Not reported & Human & None observed & None & [213] \\
\hline Phenytoin & PHE & Not reported & Human & $\begin{array}{l}\text { Congenital } \\
\text { hydantoin syndrome, } \\
\text { neurodevelopmental delay }\end{array}$ & Oxidative stress & {$[204,210]$} \\
\hline
\end{tabular}

concentrations inhibiting neural differentiation in the ESTn, transcriptomics profiles were investigated at a concentration inhibiting neurite outgrowth in the ESTn as well as a $\sim 30$-fold lower concentration inducing no morphological effects. The present study reveals common and unique gene expression profiles between the six compounds tested and provides clues for the distinction between transcriptomics responses of toxic and non-toxic concentrations that may relate to adaptive versus adverse outcomes.

\section{@̊?}

\section{Materials and Methods}

\section{Culture Media}

Complete medium (CM) contained Dulbecco's modified eagle's medium (DMEM) (Gibco BRL, Gaithersburg, MD, USA) supplemented with $20 \%$ fetal bovine serum (Hyclone, Logan, UT, USA), 1\% nonessential amino acids (Gibco BRL, Gaithersburg, MD, USA), 1\% penicillin/streptomycin (Gibco BRL, Gaithersburg, MD, USA), 2 mM l-glutamine (Gibco BRL, Gaithersburg,
MD, USA) and $0.1 \mathrm{mM}$ mercapto-ethanol (Sigma-Aldrich, Zwijndrecht, The Netherlands). Low serum medium (LS) had the same composition as CM, except that the serum percentage was $10 \%$. Insulin-transferrin-selenitefibronectin medium (ITS) contained DMEM/Ham's nutrient mixture F12 (DMEM/F12)medium (Gibco, BRL, Gaithersburg,MD, USA) supplemented with $0.2 \mu \mathrm{g} / \mathrm{ml}$ bovine insulin (Sigma-Aldrich, Zwijndrecht,The Netherlands), $1 \%$ penicillin/streptomycin (Gibco BRL, Gaithersburg,MD, USA), $2 \mathrm{mM}$ l-glutamine (Gibco BRL, Gaithersburg, MD, USA), $30 \mathrm{nM}$ sodium selenite (Sigma-Aldrich, Zwijndrecht, The Netherlands), $50 \mu \mathrm{g} / \mathrm{ml}$ apo-transferrin (Sigma-Aldrich, Zwijndrecht, The Netherlands) and $2.5 \mu \mathrm{g} / \mathrm{ml}$ fibronectin (Invitrogen, Carlsbad, CA, SA). N2 medium contained DMEM/F12 medium (Gibco,BRL, Gaithersburg, MD, USA) supplemented with $0.2 \mu \mathrm{g} / \mathrm{ml}$ bovine insulin (Sigma-Aldrich, Zwijndrecht, The Netherlands), 1\% penicillin/ streptomycin (GibcoBRL, Gaithersburg, MD, USA), $30 \mathrm{nM}$ sodium selenite (Sigma-Aldrich, Zwijndrecht, The Netherlands), and $50 \mu \mathrm{g} / \mathrm{ml}$ apo-transferrin (Sigma-Aldrich, Zwijndrecht, The Netherlands), $20 \mathrm{nM}$ progesteron (SigmaAldrich, Zwijndrecht, The Netherlands) and $100 \mu \mathrm{M}$ putrescine (SigmaAldrich, Zwijndrecht, The Netherlands). 


\section{Test Compounds}

The following compounds were used in experiments. Acetaldehyde $199 \%$ purity) (ACE) (CAS number 75-07-0, Sigma-Aldrich, Zwijndrecht, The Netherlands), carbamazepine (CBZ) (CAS number 298-46-4, Sigma-Aldrich, Zwijndrecht, The Netherlands), flusilazole (FLU) (CAS number 85509-19-9, Sigma-Aldrich, Zwijndrecht, The Netherlands), monoethylhexylphthalate (MEHP) (CAS number 4376-20-9), Wako Chemicals GmbH, Neuss, Germany), penicillin G sodium salt (PENG) (CAS number 69-57-8, Sigma-Aldrich, Zwijndrecht, The Netherlands) and phenytoin (99\% number 57-41-0, Sigma-Aldrich, Zwijndrecht, The Netherlands), were diluted in dimethyl sulfoxide (DMSO), and cell cultures (including controls) were exposed to a final DMSO concentration of $0.1 \%$.

\section{Embryonic Stem Cell Culture and Neural Differentiation}

Mouse embryonic stem cells (ESC) (ES-D3, ATCC, Rockville, MD, USA) were routinely sub-cultured every 2-3 days and grown on 35-mm dishes (Corning Incorporated, Corning, NY, USA) as a monolayer in Dulbecco's Modified Eagle Medium (DMEM)-based (Gibco BRL, Gaithersburg, MD, USA) medium supplemented with leukemia inhibiting factor (LIF) (Chemicon, Temecula, CA, USA) at a final concentration of 1000 units $/ \mathrm{ml}$. The cells were maintained in a humidified atmosphere at $37^{\circ} \mathrm{C}$ and $5 \% \mathrm{CO}_{2}$. Induction of neural differentiation was performed as described earlier [142, 177].

In brief, ESC suspensions (3.75 $310^{4}$ cell $/ \mathrm{ml}$ ) were placed on ice before the initiation of the culture. Drops $(20 \mu \mathrm{l})$ containing 750 cells in CM were placed onto the inner side of the lid of a $90-\mathrm{cm}$ Petri dish filled with PBS (Gibco BRL, Gaithersburg,MD, USA) and incubated at $37^{\circ} \mathrm{C}, 90 \%$ relative humidity, and $5 \% \mathrm{CO}_{2}$. After 3 days of hanging drop culture, embryoid bodies (EB) had formed and were subsequently transferred to $60-\mathrm{mm}$ bacterial Petri dishe (Greiner Bio-one, Frickenhausen, Germany) containing CM supplemented with $0.5 \mu \mathrm{M}$ retinoic acid (RA). On day 5, EB were plated on laminin (Roche, Basel, Switzerland) coated 35-mm dishes (Corning Incorporated, Corning, NY) in LS medium supplemented with $2.5 \mu \mathrm{g} / \mathrm{ml}$ fibronectin (Invitrogen, Carlsbad, CA USA). One day later, on day 6 , the LS medium was replaced by ITS medium. On day 7, EB were washed with PBS and incubated in cell dissociation buffer (Gibco BRL, Gaithersburg, MD, USA) for three minutes. Then EB were carefully detached from each Petri dish without dissociating the EB, and the entire content was replated on poly-L-ornithine (Sigma-Aldrich, Zwijndrecht, The Netherlands) and laminin coated dish in $\mathrm{N} 2$ medium supplemented with $10 \mathrm{ng} / \mathrm{ml}$ basic fibroblast growth factor (bFGF; Srtathmann-Biotec AG, Englewood, CO). The N2 medium supplemented with bFGF was replaced every other day for 7 days.

\section{Resazurin cell viability assay}

To determine compound effects on cell viability, a Resazurin dye reduction assay (Promega, Leiden, The Netherlands) was used as a measure for the number of viable cells. ESC were plated in 96-well plates at 500 cells per well in $\mathrm{CM}$ supplemented with LIF and allowed to attach for $2 \mathrm{~h}$ before compound exposure was started in the following concentration ranges $(n=6$ per concentration): ACE $3-1000 \mu \mathrm{M}$, CBZ $3-600 \mu \mathrm{M}$, FLU $0.01-300 \mu \mathrm{M}$, MEHP $30-1000 \mu \mathrm{M}$, PenG $3-1000 \mu \mathrm{M}$ and PHE $0.3-100 \mu \mathrm{M}$. As a positive control in the viability assay, 5 -fluorouracil was tested at $0.15 \mu \mathrm{M}$. Concentration ranges were based on earlier concentration-response range finding studies in the same model with which the range between no cytotoxicity and complete cell death was determined (data not shown). The maximum concentration tested was either $1000 \mu \mathrm{M}$ or based on the solubility of the compound in the medium. Cells were subsequently cultured for 5 days at $37^{\circ} \mathrm{C}, 90 \%$ relative humidity, and $5 \% \mathrm{CO}_{2}$, with a medium renewal containing compound for exposure on day 3. On day 5 (approximately $80 \%$ cell confluence in non-exposed controls), CellTiter-blue (Promega, Leiden, The Netherlands) was added to each well and incubated for $2 \mathrm{~h}$. After the incubation period, fluorescence was read using a FLUOstar spectrofluorometer (FLUOstar Optima, BMG Labtech, de Meeren, The Netherlands) at $544 \mathrm{~nm}$ (excitation) and $590 \mathrm{~nm}$ (emission). Three independent experiments were performed. Concentration-response analysis to determine the $20 \%$ maximal inhibitory concentration (IC20) for cytotoxicity was performed using PROAST (Possible Risk Obtained from Animal Studies software (RIVM, Bilthoven, The Netherlands), a freeware program with which concentration-response curves can be fitted and benchmark concentrations can be determined (http://www.rivm.nl/en/Library/Scientific/Models/PROAST).

\section{Treatment and morphological scoring for effects on neural} outgrowth

Highest concentrations tested in the transcriptomics experiment were selected on the basis of 1) concentration range finding studies in the ESTn (data no shown) providing an inhibition of approximately 50\% reduction in neural outgrowth, 2) the Resazurin viability assay, wherein the highest concentration provided a minimum viability of $80 \%$ (IC20), or 3) a highest dose of $1000 \mu \mathrm{M}$, chosen as the highest concentration feasible in vivo. The lowest concentration tested in the transcriptomics experiment was always a factor 30 lower compared to the highest concentration tested. The highest concentrations tested for ACE, CBZ, MEHP and PHE were equal to the concentration resulting in 50\% reduction of neural outgrowth (ID50) (ACE $30 \mu \mathrm{M}$; CBZ $100 \mu \mathrm{M}$; MEHP 100 $\mu \mathrm{M}$ and PHE $30 \mu \mathrm{M})$, the highest concentration for FLU was equal to the IC20 
concentration $(10 \mu \mathrm{M})$ and PENG was tested at a highest concentration of $1000 \mu \mathrm{M}$. For morphological assessments, compounds were tested at the following concentrations, of which the lowest and highest concentrations were included in the transcriptomics experiment: ACE 1, 3, 10 and $30 \mu \mathrm{M}$; CBZ 3 , 10,30 and $100 \mu \mathrm{M}$; FLU 0.3, 1, 3 and $10 \mu \mathrm{M}$; MEHP 3, 10, 30 and $100 \mu \mathrm{M}$; PENG 30, 100, 300 and $1000 \mu \mathrm{M}$ and PHE 1, 3, 10 and $30 \mu \mathrm{M}$. Control EB were treated with $0.1 \%$ DMSO. The ESTn and morphological scoring were performed as described earlier [142, 177]. For morphological scoring, cultures were treated for $72 \mathrm{~h}$ from initiating suspension culture on day 3 of the protocol until the start of the serum free period on day 6. Morphological effects were determined by assessment of the extent of neurite outgrowth from the EB, at the end of the differentiation protocol (day 11), observed using an IX51 inverted microscope (Olympus, Zoeterwoude, The Netherlands) with CellD software (Olympus, Zoeterwoude, The Netherlands). Morphology of neura outgrowth was scored as the percentage of neural corona surrounding each $\mathrm{EB}$, irrespective of the distance of outgrowth from the EB, as was described earlier [142]. For scoring neurite outgrowth, a cutoff was chosen at $\geq 75 \%$ neural outgrowth around the EB, based on historical control data. Thus, when more than $75 \%$ of the corona around an EB contained microscopically observable neural outgrowth, the EB was scored positive, whereas below $75 \%$ it was scored negative. Three $35-\mathrm{mm}$ Petri dishes per concentration were scored (containing approximately $40 \mathrm{~EB}$ per dish) and each dish was regarded as one independent observation. A concentration-range finding study was performed for each compound to determine half maximal inhibitory concentration of differentiation (ID50) on neural differentiation, consisting of three separate experiments. Within each experiment three dishes per concentration were scored (with $40 \mathrm{~EB}$ per dish). Statistical analysis to determine the ID50 on neural differentiation was performed using PROAST.

During the final gene expression experiment a concentration-response study was performed in parallel for each compound to determine ID50 on neural differentiation in this specific experiment using PROAST. For gene expression analysis, parallel cultures were harvested at earlier time points (see next section).

\section{RNA isolation and whole-genome expression profiling}

Exposed differentiation cultures were treated from day 3 of the protocol onwards and sampled after $24 \mathrm{~h}$ exposure (day 4) (eight replicates per group). The $24 \mathrm{~h}$ exposure was chosen as the time point expressing the least variability between samples as observed in our earlier time-course study with the neurodevelopmental toxicant $\mathrm{MeHg}$ [177]. RNA from control differentiation cultures was sampled on protocol days 3 (eight replicates), 4 (five replicates), and 5 (five replicates). Gene expression changes over time correlated well with previous studies, both qualitatively and quantitatively [177]. Cells (approximately 30-40 EB/sample) were directly collected in RNA Protect (Qiagen Benelux, Venlo, The Netherlands) to stabilize RNA, and total RNA was purified using the Qiagen RNeasy Plus Mini Kit (Qiagen Benelux, Venlo, The Netherlands) following manufacturer's instructions. RNA quantity was determined using the NanoDrop Spectrophotometer (Isogen Lifescience, de Meern, The Netherlands). RNA integrity was assessed on the 2100 Bioanalyzer (Agilent, Amstelveen, The Netherlands) using the RNA 6000 Nano Chip Kit (Agilent, Amstelveen, The Netherlands), and RNA with RNA integrity number (RIN) $>8.0$ was used for gene expression analysis. Gene expression analysis using the Mouse Genome 4302.0 arrays (Affymetrix, Santa Clara, CA, USA) was performed as described previously [177]. Quality controls, including scaling factors, average intensities, present calls, background intensities, noise, and raw $\mathrm{Q}$ values, were performed according to the manufacturer's instructions and showed to be within acceptable limits for all chips. Raw and normalized data are available at ArrayExpress (http://www.ebi.ac.uk/arrayexpress/) under accession number E-TABM-1216.

\section{Data Analysis and Statistics}

Affymetrix CEL files were normalized using the Robust Multichip Average (RMA) algorithm [158] using RMAexpress [159]. For probe to gene mapping, a custom Chip Description File (CDF) was used according to the assembly by de Leeuw et al. [160] (http://mad-db.science.uva.nl/ wdeleeuw/HybridAnnot/ version6.html). Of the hybrid probe-set definitions included in the custom annotation, 16,331 probe sets defined by the Brainarray custom CDF version 11 (http://brainarray.mbni.med.umich.edu/Brainarray/Database/CustomCDF) [161] and 4648 additional probe sets defined by Affymetrix chip annotation 26 were used in further analyses, giving a total of 20,979 probe sets. Probe sets for Affymetrix internal controls or probe sets that did not correspond to an Entrez Gene ID were not used in further analyses. Statistical analyses were carried out using the R statistical software environment (http://www.R-project. org) using log-transformed values. For each gene, maximal fold change (FC) in gene expression between the experimental groups were determined by comparing the average normalized signal values per group and were calculated as the maximum/minimum ratio. For compound toxicity effects, the treated samples were compared to the time matched day 4 controls. Genes differentially expressed between any of the experimental groups (two concentrations and control) were identified by a one-way ANOVA. A significance threshold of FDR $<0.05$ was used to select genes that were significantly differentially expressed due to compound exposure. Functional annotation and enrichment 
for Gene Ontology (GO) biological processes were studied using DAVID (http://david.abcc.ncifcrf.gov/) [162]. Significantly enriched GO categories were based on $\mathrm{p}<0.01$.

For pathway-level analysis, the expression of each gene was corrected for its timed control after which Tox-Profiler [99] was used to score the difference between the mean expression level of GO biological processes and that of the remainder of the 20,979 probe sets defined earlier. Gene sets were significantly regulated if $\mathrm{T}>4.0$ and $\mathrm{E}<0.05$. The $\mathrm{T}$-value is the value obtained by a T-test between the expression changes for a defined set of genes versus all other genes. The corresponding formula and other calculation details can be found in Boorsma et al., 2005 [99]. The E-value is the associated two-tailed P-value with Bonferroni correction for the number of gene sets tested.

Hierarchical clustering of changes to control for all significantly differentially expressed genes (or subsets thereof selected on the basis of GO-terms) was performed in GeneMaths XT (Applied Maths, Sint-Martens-Latem, Belgium), using Euclidean distance and Ward linkage. Venn diagrams describing relations of significant gene expression were created using Venny [214].

The effect of the highest concentration analyzed for gene expression changes per compound on gene expression was further studied using principal component analysis (PCA) by means of the differentiation track algorithm, as was described earlier [177, 187]. In this analysis, a principal component is defined as a mathematically derived combination of genes and their expression characteristics which can be used to depict part of the process observed. A number of principal components that are mutually independent can be derived which in combination describe the process under study. For the differentiation track algorithm, at first a gene list was defined, which comprised the genes that were identified to be significantly differentially expressed among mESC neura differentiation over time sampled at days 3,4 and 5 of the mESC neural differentiation protocol. PCA analysis was performed with these genes using R statistical software, resulting in a curve describing the differentiation track. A differentiation track derived on the basis of genes showing differentia expression between day 3, day 4 and day 5 (FDR $<0.01, \mathrm{FC}>1.5$ ) in the ESTn was used to describe compound effects on the track at day 4 (after $24 \mathrm{~h}$ exposure). Limiting the analysis to genes showing a time-related change in expression in controls avoided the contribution of genes not regulated during neural differentiation in the ESTn. Coordinates along the first and second principal components were calculated for each sample.

\section{ठำ}

\section{Results}

Concentration-dependent compound effects on viability and neurite outgrowth

ACE, CBZ, FLU, MEHP and PHE perturbed pluripotent mESC viability in a concentration-dependent manner, whereas PENG did not have an effect on cell viability up to a concentration of $1000 \mu \mathrm{M}$ (Figure 1). Compound concentrations reducing cell viability by $20 \%$ (IC20) were $80.4 \mu \mathrm{M}$ for ACE, $204.5 \mu \mathrm{M}$ for CBZ, $12.9 \mu \mathrm{M}$ for FLU, $572.4 \mu \mathrm{M}$ for MEHP and $35.1 \mu \mathrm{M}$ for PHE. On day 11 in the assay, ACE, CBZ, MEHP and PHE significantly altered neura differentiation in the ESTn at the highest concentration tested (Figure 1). FLU and PENG did not significantly impact neural differentiation at concentrations tested.

Genes and GO-biological processes regulated by compounds Compound-induced differential gene expression was compared between exposed mESC neural differentiation cultures and time-matched contro cultures, using one-way ANOVA (FDR $<0.05)$. Test concentrations were at the ID50 for positive compounds or at the highest concentration tested for negative compounds, as well as concentrations 30 fold lower for each compound. Concentration-dependently, 1024, 687, 1574, 4, 3 and 55 genes were identified to be significantly regulated by ACE, CBZ, FLU, MEHP, PENG and PHE, respectively (Table $2 \mathrm{~A}$ ). For all compounds tested, the number of genes significantly differentially expressed was highest at the high concentration tested, as determined by post-hoc analysis. The most responsive compounds, ACE, CBZ and FLU showed significant commonalities in genes differentially expressed (for instance, ACE and FLU commonly regulated 339 genes) (Table 2a). In total, 2639 unique genes were regulated by any of the compounds at any concentration tested. Hierarchical clustering of FC ratios for these 2639 genes provided specific concentration-dependent gene expression patterns for each compound (Figure 2).

\section{Distribution of regulation of GO-terms among compounds}

Enrichment of GO biological processes $(\mathrm{p}<0.01)$ by gene sets derived per compound was observed for ACE, CBZ and FLU. However, due to a limited number of genes regulated, MEHP, PENG and PHE exposure did not result in enrichment of any GO biological processes (Table 2B). Given differential gene expression changes across compounds, functional analysis using Tox-profiler was performed to identify significant quantitative changes at the level of GO-terms (Figure 3). By at least one compound and concentration of any of 
A

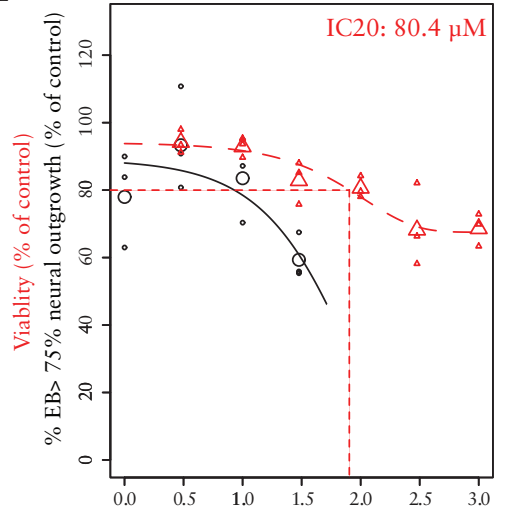

$\log 10-$ Concentration Acetaldehyde $(\mathrm{\mu M})$

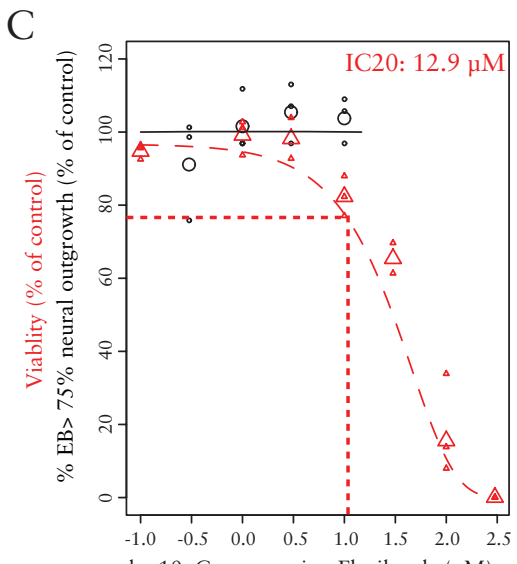

10-Concentration Flusilazole ( $\mathrm{mM})$
B

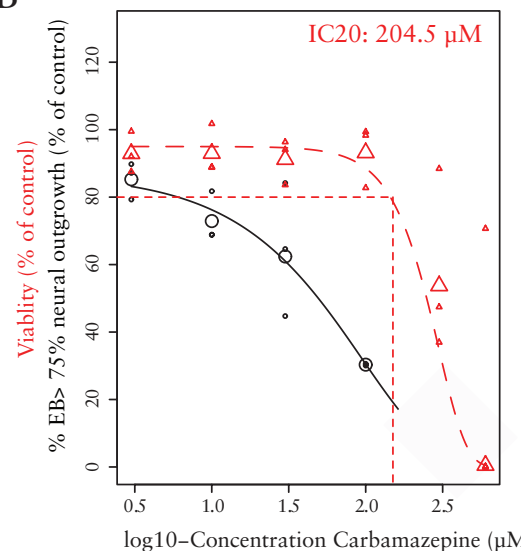

-Concentration Carbamazepine $(\mathrm{\mu M})$

$\mathrm{D}$

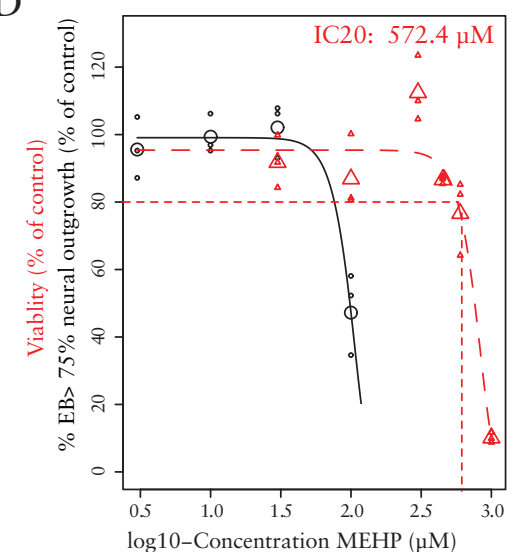

$\log 10-$ Concentration MEHP $(\mathrm{\mu M})$

Figure 1 Compound effects on viability (Resazurin cell viability assay) and morphological neuron outgrowth (ESTn).Concentration-response effects on cell viability in $\mathrm{mESC}$ (triangles) and concentration response effects on the percentage of EB with $>75 \%$ neural outgrowth (circles). Smaller circles and triangles represent separate experiments. The large circles and triangles represent the geometric mean of experiments at the specific concentration. Curves were fit using PROAST software.

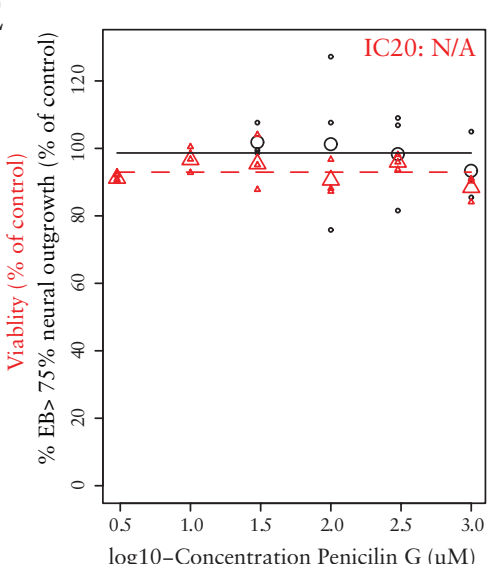

F

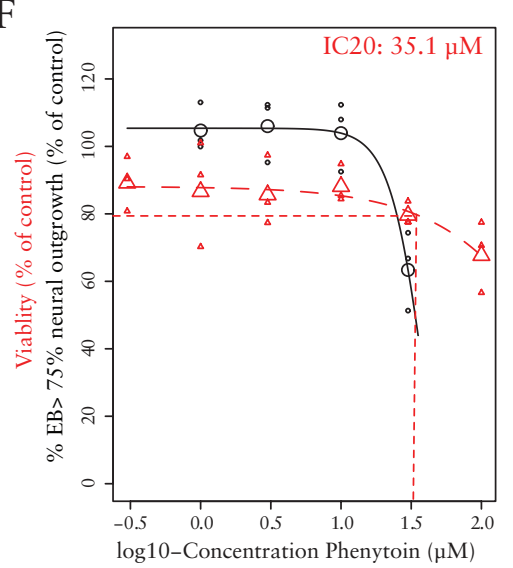

Figure 1 Continued

the six compounds, we identified 136 enriched GO-terms $(\mathrm{T}>4, \mathrm{E}<0.05)$ Hierarchal clustering of enriched GO-terms produced three clusters of expression profiles and $\mathrm{GO}$-term themes (indicated as clusters $\mathrm{A}, \mathrm{B}$ and $\mathrm{C}$ in Figure 3). In general, GO-terms in cluster A were mostly up-regulated in a concentration dependent manner by the majority of the six compounds (Figure 3A). For example, MEHP and PENG significantly regulated ion transport and oxidation reduction and FLU significantly regulated sterol- and cholesterol biosynthetic processes (FLU). GO-terms in cluster B were all down-regulated by compound exposures (Figure 3B). For instance, PHE down-regulated GO-terms in this cluster related to kinase activity, cell division, phosphorylation, ubiquitination and apoptosis, while FLU, MEHP, PENG and PHE down-regulated genes related to transcription. $\mathrm{GO}$-terms in cluster $\mathrm{C}$ were up- or down-regulated in a compound- and concentration-dependent manner and mainly consisted of GO-terms related to development $(60 \%)$ and transcription (14\%). GO-terms were up-regulated by ACE and CBZ, and down-regulated by FLU, MEHP, PHE, whereas PENG only showed a limited number of GO-terms being significantly regulated related to transcription (Figure 3C). All terms in cluster $\mathrm{C}$ were upregulated by ACE, representing a broad range of general and specific organ development related GO-terms. MEHP and PHE both down-regulated general development and transcription related GO-terms, whereas MEHP specifically affected genes related to skeletal development and PHE specifically affected genes present in cardiovascular development and cell differentiation related GO-terms. 


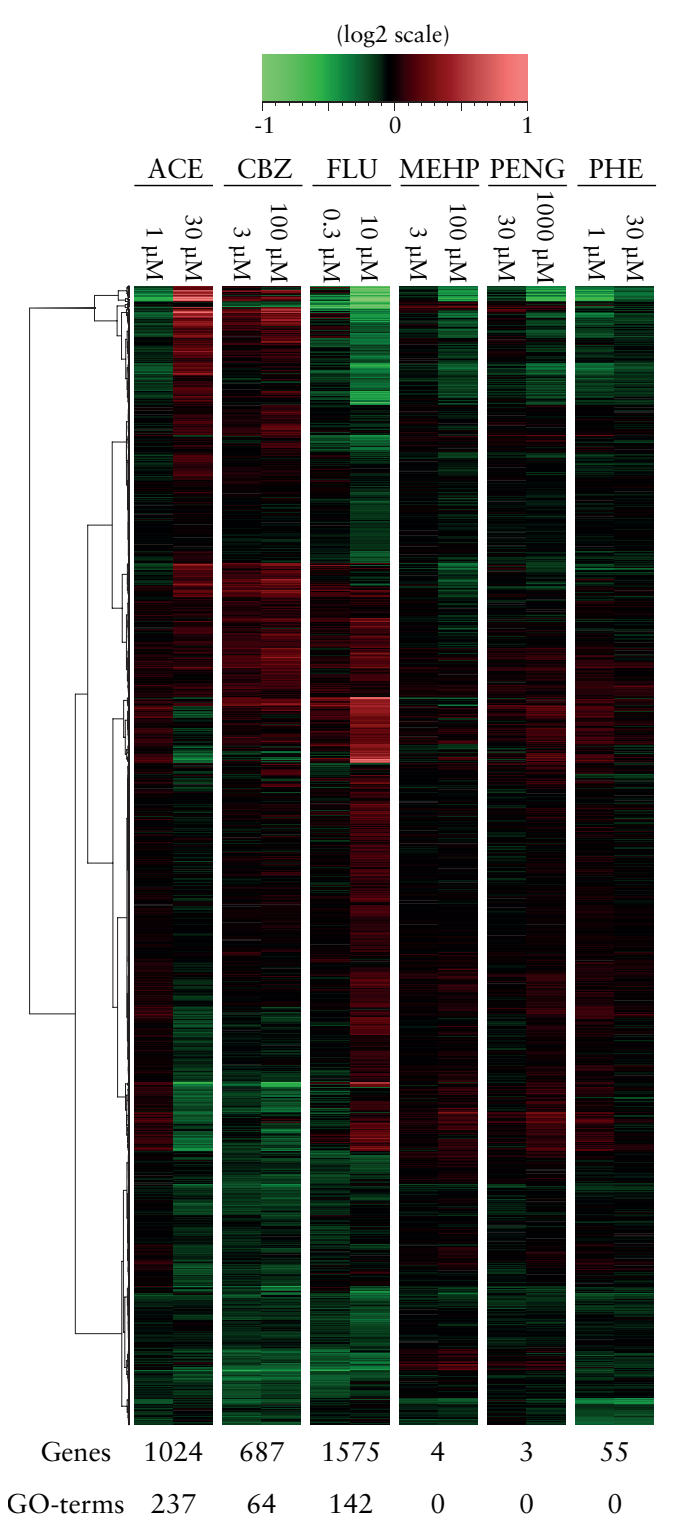

Figure 2 Hierarchal clustering plot of all 2639 genes regulated concentration-dependently by any of the compounds (One-way ANOVA FDR $<0.05, \log 2$ scale). Number of genes regulated by each compound and number of $\mathrm{GO}$ biological processes enriched by these genes are presented under the hierarchal clustering.

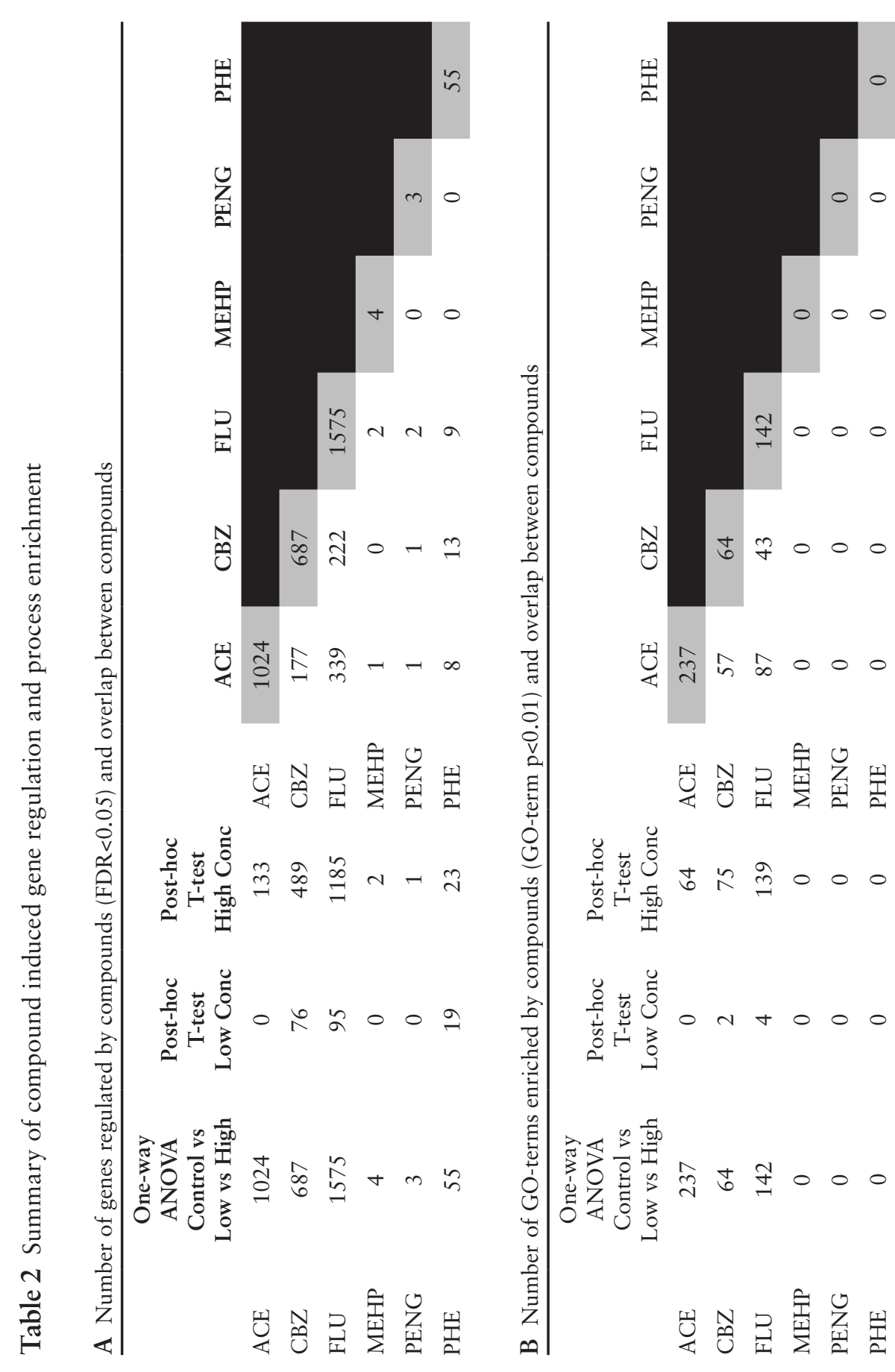




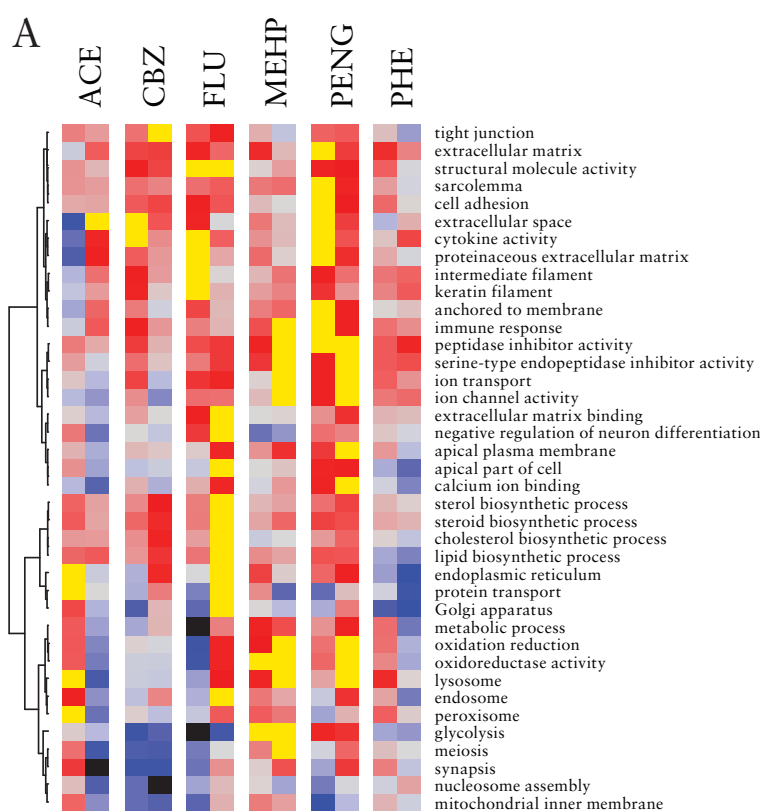

B

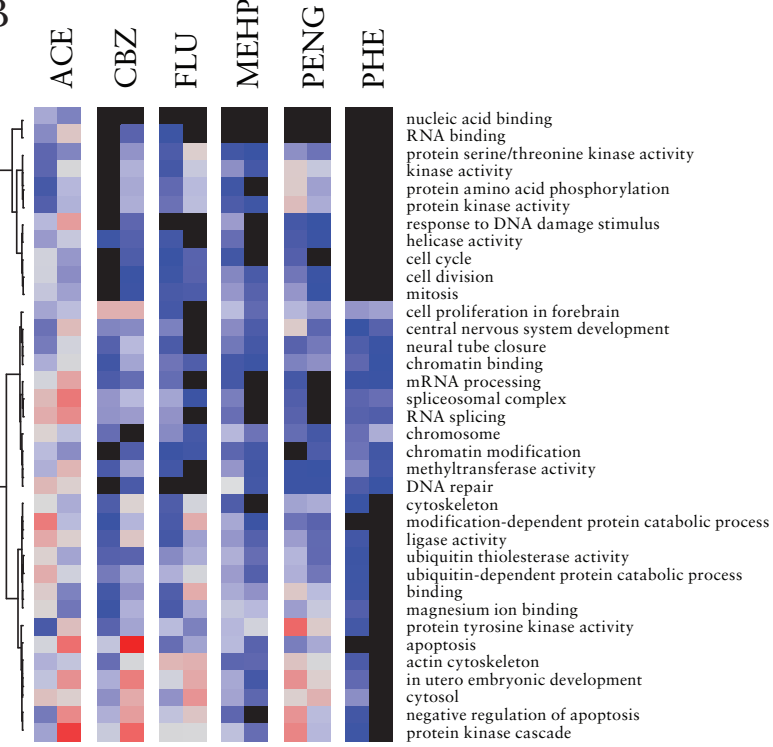

Figure 3 Hierarchal clustering plot of T-values describing gene distribution shifts within GO biological processes. (White with grey lining = significantly up-regulated $(\mathrm{T}>4, \mathrm{E}<0.05), \mathrm{Black}=$ significantly down-regulated $(\mathrm{T}<-4, \mathrm{E}<0.05)$, blue to grey to red: not-significant gradient of T-values).

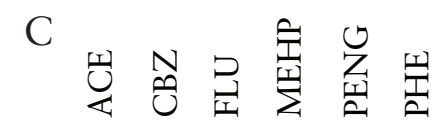
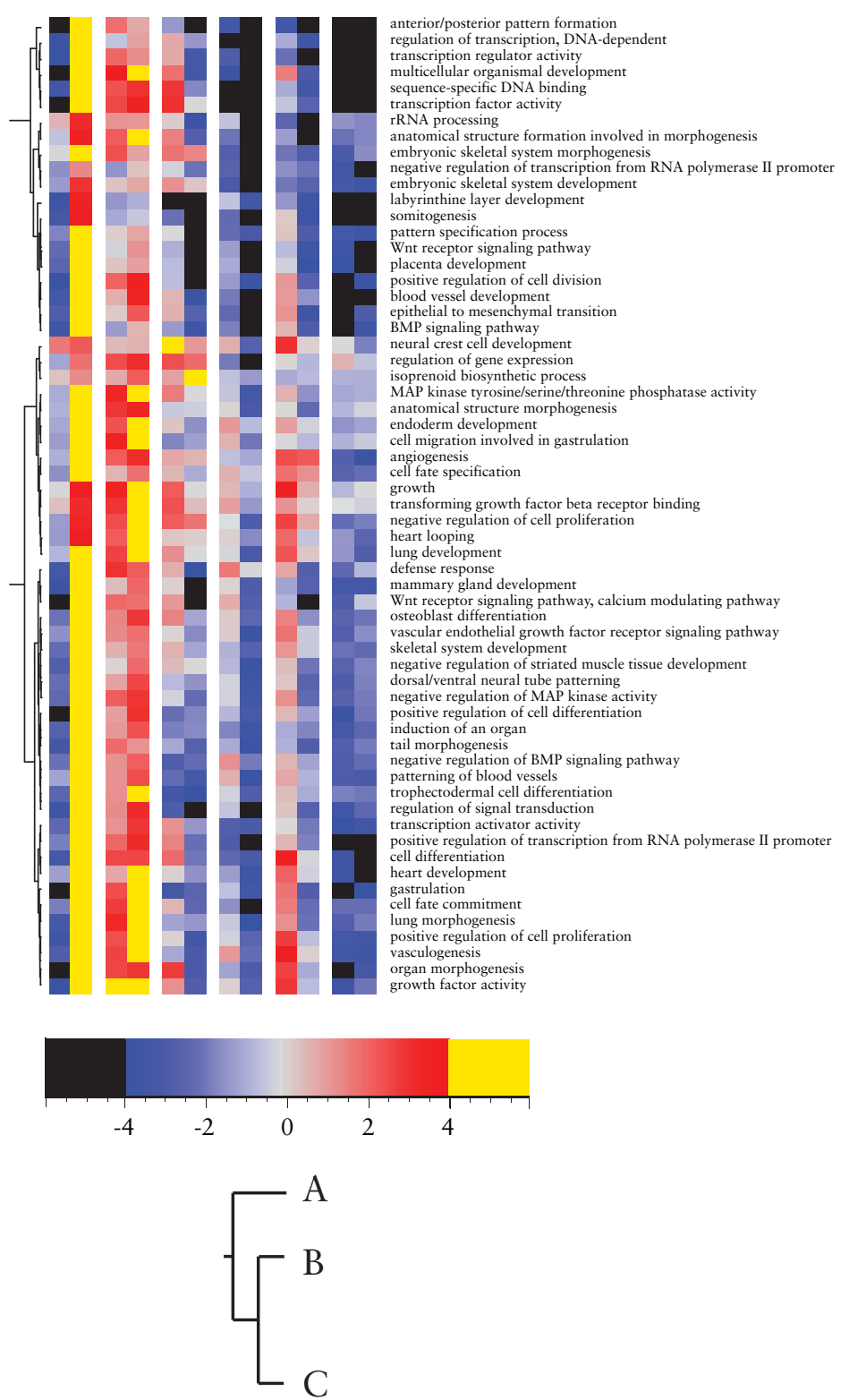

Figure 3 Continued 


\section{Direction of gene expression within selected GO biological} processes

We further evaluated gene expression changes within selected compound(s)specific enriched GO biological processes relevant for development. These included embryonic morphogenesis (Figure 4A), neuron differentiation (Figure $4 \mathrm{~B})$, and wnt receptor signaling pathway (Figure 4C). In general, genes within these three processes were primarily regulated only after exposure to ACE, CBZ or FLU. Most genes associated with these processes were up-regulated by ACE and CBZ when down-regulated by FLU, and vice versa. For example, Pax6 was down-regulated by ACE and CBZ at the high concentration, but up-regulated by the high concentration FLU (Figure 4B). As a trend, MEHP, PENG and PHE gene expression direction followed FLU expression profiles, but changes were not significant due to variation between samples. In addition, the GO-term sterol metabolic process was analyzed as a known specific target for flusilazole (Figure 4D). The GO biological process sterol metabolic process, was specifically significantly enriched by FLU and not by the other compounds. FLU up-regulated the expression of genes related to sterol metabolism (18 out of 19 genes).

Compound induced deviation from the differentiation track

Compound effects on gene expression related to differentiation were visualized by PCA using the differentiation track algorithm (Figure 5). Genes significantly differentially expressed (FDR $<0.01, \mathrm{FC}>1.5$ ) over time in control differentiation culture on days 3, 4 and 5 (4006 genes) were used to derive the differentiation track. Using these genes, for the location of each compound in the PCA was determined. In figure 5A, each separate control sample was plotted in the PCA and for exposed samples the mean of all samples $(n=8)$ was shown. A magnification of the PCA is shown in figure $5 \mathrm{~B}$ to highlight the deviation of the compound exposed samples from the day 4 control. ACE and CBZ findings indicated reduced differentiation on the track as compared to controls and PENG, whereas the remaining compounds tended to indicate enhanced differentiation (to the lower right of the average day 4 control). The grouping of these compounds by PCA is comparable to the directional differences observed in gene expression changes.

Low versus high concentration comparison for CBZ and FLU Differences in compound effects on gene expression and GO biological process enrichment at the non-toxic versus differentiation-inhibiting concentrations tested were performed for CBZ and FLU. For CBZ of the 76 and 489 genes regulated at the low and high concentrations, respectively, 47 genes were

$$
* *
$$

\section{덩}

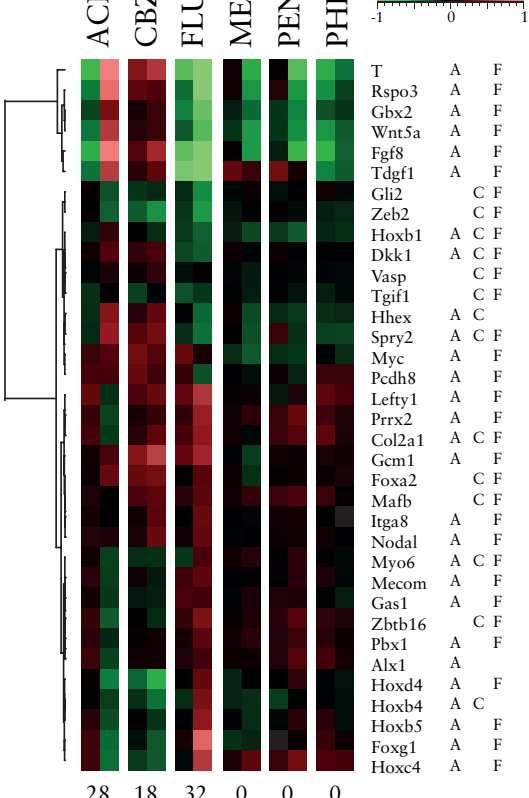

$\begin{array}{lll}28 & 18 & 32 \\ (41) & (14) & (47)\end{array}$
Embryonic Morphogensis
B

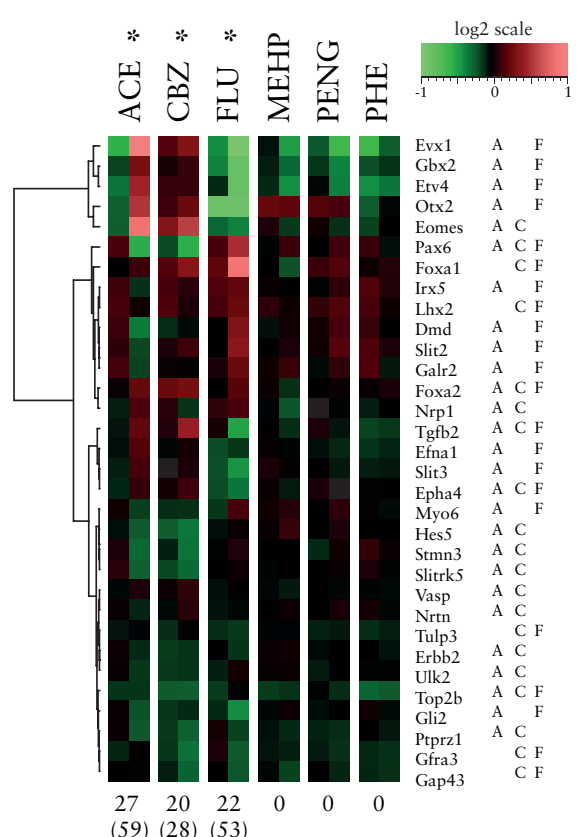

$\begin{array}{lll}27 & 20 & 22 \\ (59) & (28) & (53)\end{array}$
Figure 4 Genes significantly concentration-dependently expressed by at least two of the six compounds compared to control (FDR $<0.05, \log 2$ scale) for the GO biological processes A) Embryonic Morphogenesis, B) Neuron Differentiation, C) Wnt receptor signaling pathway and expressed by at least one of six compounds for D) Sterol Metabolic Process. Genes significantly changed are marked with A (ACE), C (CBZ) or $\mathrm{F}$ (FLU). No genes were significantly regulated by MEHP, PENG or PHE in any of the GO biological processes. Significant enrichment of the GO biological process by a compound as determined by DAVID is marked with * and significant distribution shif as determined by Tox-Profiler is marked with \#. Number of genes regulated in the figure and in the GO biological process (in brackets) are displayed under each column. 
C Wnt Receptor Signalling Pathway

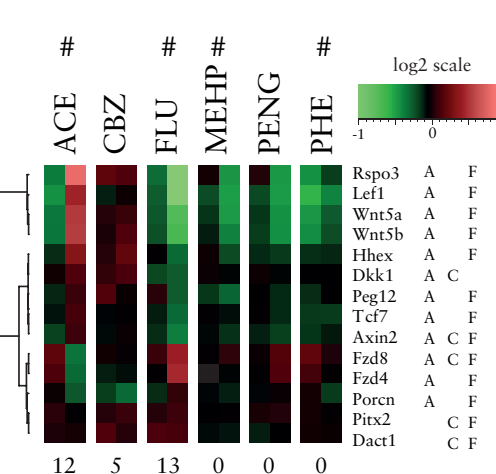

$\begin{array}{ccc}12 & 5 & 13 \\ (21) & (8) & (21)\end{array}$

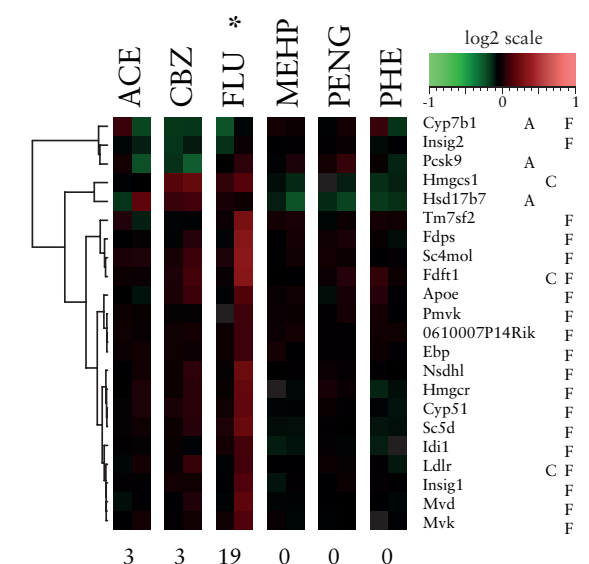

Figure 4 Continued

regulated at both concentrations (Figure 6A). FLU significantly regulated 95 and 1185 genes at the low and high concentration, respectively, with 62 genes regulated at both concentrations (Figure 6B). CBZ enriched 2, 4 and 113 GO biological processes for low-specific, common and high-specific genes, respectively (Figure 6C). GO biological processes significantly enriched $(\mathrm{p}<0.05)$ by the low-specific and common genes included processes involved in methylation (histone methylation, chromosome modification) and transcription. For transcription, significance values were concentration-dependently increased, whereas for methylation related GO biological processes they were not. At the high concentration CBZ significantly regulated processes involved in general and organ development, neural development, transcription, cellular processes and metabolism. FLU enriched 0,7 and 256 GO biological processes for low-specific, common and high-specific genes, respectively (Figure 6D). GO biological processes significantly enriched by the low-specific genes included processes involved in transcription and glucose metabolism. High-concentration specific significantly regulated genes related to enriched processes involved in general and organ development, neural development, transcription, cellular processes, metabolism and apoptosis.

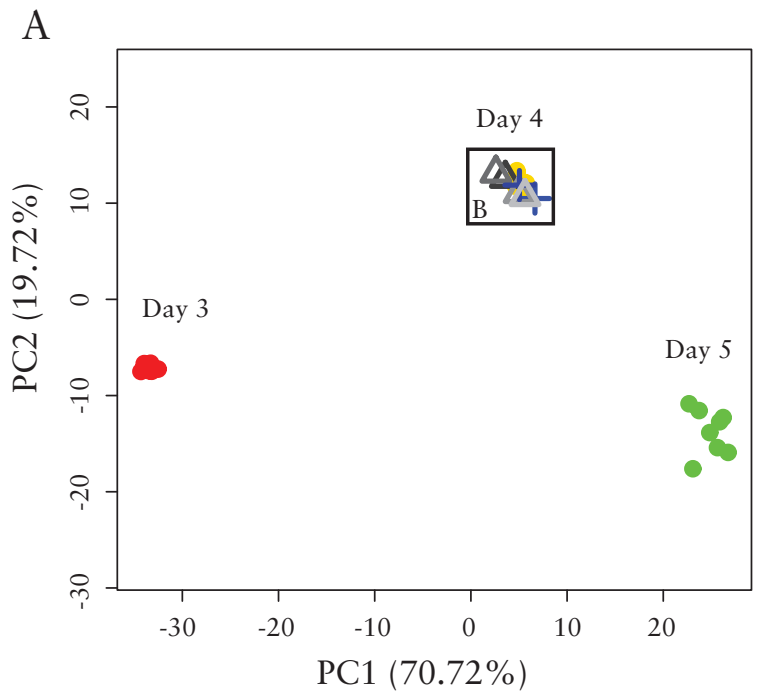

B

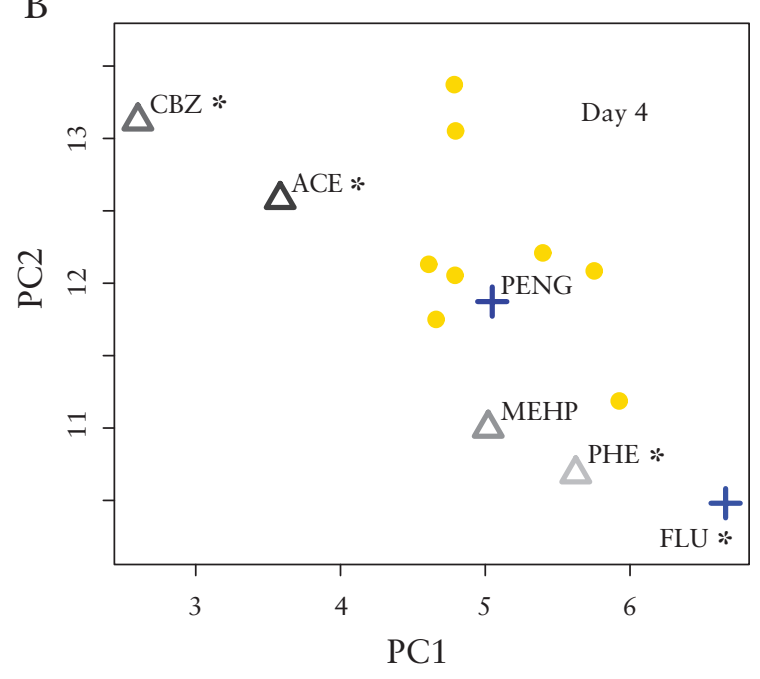

Figure 5 Principal component analysis (PCA) presenting compound deviation from the differentiation track. A) PCA plot based on the 4006 genes significantly regulated over time on days 3 (red), 4 (yellow) and 5 (green) of the neural differentiation protocol ( $\mathrm{FDR}<0.01$ and $\mathrm{FC}>1.5$ ). Compound exposed cultures (mean of $\mathrm{n}=8$ samples, triangles (in vivo neuro-developmental toxicants) and crosses (in vivo non-neuro-developmental toxicants)) are plotted in the PCA using the 4006 genes regulated over time. B) Magnification of the day 4 control samples and exposed samples $(*=$ Compounds significantly deviating from the day 4 control $(\mathrm{p}<0.05))$. 
A

B

FLU

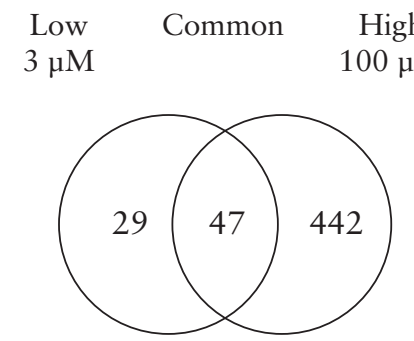

C

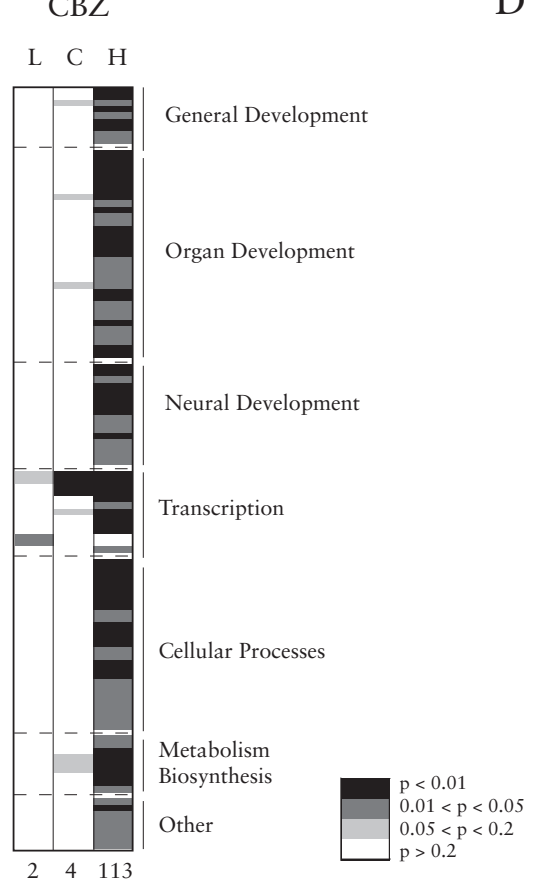

D FLU
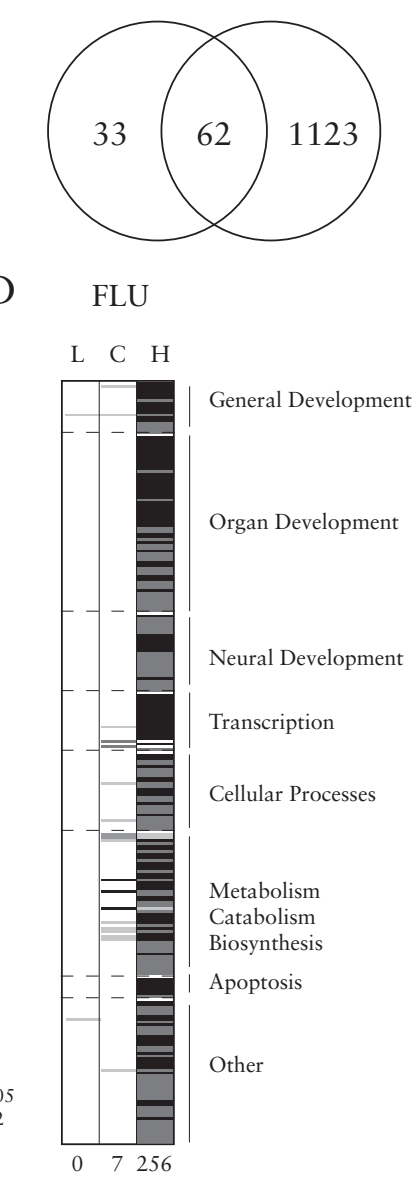

Figure 6 Venn diagrams and hierarchal clustering plots describing overlap of genes significantly expressed compared to control (One-way ANOVA with post hoc t-test $($ FDR $<0.05))$ at the low and high concentration tested. Venn diagrams present the number of genes regulated by the low concentration only, the high concentration only or both concentrations for A) CBZ ( 3 and $100 \mu \mathrm{M})$ and B) FLU $(0.3$ and $10 \mu \mathrm{M})$ ). Hierarchal clustering plots describe GO-biological processes significantly enriched $(\mathrm{p}<0.05$, ranked by general theme and by number changed) by low concentration only (L), the high concentrations only $(\mathrm{H})$ or both concentrations $(\mathrm{C})$ for $\mathrm{C}) \mathrm{CBZ}$ and $\mathrm{D})$ FLU.

\section{Discussion} E recently developed a neural differentiation variant of the classic cardiac
EST, the ESTn, aimed at predicting neurodevelopmental toxicity, and conducted a series of studies investigating the potential implementation of transcriptomic analyses in the assay [142,177]. Our data suggest that transcriptomics implementation in the ESTn may provide added value information on compound-related mechanisms of action over time and concentration, which may improve predictivity. In the present study, we further examined the relationship between concentration-dependent transcriptomic responses and neural differentiation in the ESTn using six mechanistically diverse compounds. PENG appeared negative as expected, and the non-neurodevelopmental toxicant FLU inhibited neural differentiation at cytotoxic concentrations only. However, ACE, CBZ, MEHP and PHE induced inhibition of neural differentiation at non-cytotoxic concentrations, in concordance with the known ability of these compounds to act as neural developmental toxicants in humans and/ or in vivo [204-207].

Equipotent concentrations were selected across compounds as determined by neural differentiation inhibition on day 11 in ESTn. In contrast, they showed different potencies as regards gene expression changes after $24 \mathrm{~h}$ exposure. Concentration-dependently, ACE, CBZ and FLU induced robust changes with $\geq 687$ genes identified to be significantly altered, $>10$ fold more compared to MEHP and PHE. These differences in gene expression changes after $24 \mathrm{~h}$ exposure may represent differing response time courses between compounds, which indicate possible differences in modes of action. Similar phenomena in compound potencies for the induction of differential gene expression have been described in the cardiac ESTc, zebrafish embryo toxicity test (ZET) and the rat whole embryo cultures (WEC) $[81,82,215]$. For example, in the ZET, for glycol-ether and triazole compounds tested at concentrations equally embryotoxic at $72 \mathrm{~h}$, glycol ethers induced $>20$ fold more genes than triazoles after 24h exposure, [215]. Consequently, dependent on the effectiveness in ESTn over time of the compound tested, the $24 \mathrm{~h}$ exposure time point may or may not be optimal for the detection by transcriptomics of neurodevelopmental toxicity in the assay. Additional time-response studies for compounds less responsive at $24 \mathrm{~h}$ may increase the sensitivity of this approach.

The present study revealed no genes regulated by all of the embryotoxic compounds tested, which is obviously caused largely by the low gene expression response to MEHP and PHE. In addition, the overlap in genes regulated 
between any two compounds was also limited. Out of the six compounds, ACE and FLU shared the most overlap in gene expression changes (339 genes). On the other hand, genes regulated by both developmental neurotoxicants ACE and CBZ (177 genes) enriched 51 GO biological processes $(\mathrm{p}<0.01)$, mainly involved in (neural) development $(\mathrm{n}=21)$ and differentiation $(\mathrm{n}=12)$. In addition, genes within these processes (for instance neuron differentiation, figure 4B) were predominantly regulated in the same direction by both compounds. In contrast, the limited response to the neurodevelopmental toxicant PHE limited the overlap between PHE and ACE or CBZ (8 and 13 genes, respectively) resulting in no common GO biological processes. The limited overlap between compound induced gene expression profiles suggests different modes of action and different toxicity pathways for the compounds used in this study. Transcriptomic studies examining multiple mechanistically diverse classes of compounds in WEC [82], ZET [216] and cardiac EST [81] support our observations of compound-induced specificity of signatures despite common morphological effects. For example, in the WEC, four compound inducing similar neural tube defects at equipotent concentrations, showed distinct gene expression profiles and enriched developmental GO biological processes [82]. Within the top 1000 regulated genes, only $10-16 \%$ of the genes overlapped between any two compounds despite all four compounds inducing neural tube defects. Thus, distinct gene expression disruption profiles may underlie similar compound induced developmental defects. Therefore, gene set biomarkers for developmental toxicity should not only be based on common responses between embryotoxicants, but complementary responses should also be considered. This notion corroborates the concept of "pathways of toxicity", as described in the trend setting US-NAS Report on toxicity testing in the $21^{\text {st }}$ century [217]. Clearly, the challenge is in defining the critical "pathways of toxicity" for developmental toxicity, for which the current study provides another set of background data.

Functionally relevant developmental pathways as represented in GO-terms appeared informative about compound-specific effects. Significant regulation was observed in the Wnt-receptor signaling pathway, important during neural tube formation [218]. In addition, embryonic morphogenesis and neura differentiation were regulated by ACE, CBZ and FLU, mainly including early development related genes ( $T$, Wnt $5 a, F g f 8$ and early Hox genes) and well known early neuroectodermal related genes such as $\operatorname{Pax} 6$ [219]. Interestingly, a number of gene clusters disrupted by ACE and $\mathrm{CBZ}$ were inversely regulated compared to FLU and to a lesser extent MEHP and PHE. On a more general level, this was also apparent in the differentiation track method, showing delayed differentiation induced by ACE and CBZ, whereas FLU and PHE treated cultures appeared ahead on the differentiation track. Similarly, as observed in the morphological test at day 11 of the ESTn, ACE and CBZ decreased neural differentiation concentration dependently, whereas FLU did not affect differentiation. These findings, observed at three distinct levels (morphology, gene expression and pathway level), further imply that different mechanisms of action are responsible for developmental toxicity between these compounds.

ACE, ethanol's active metabolite which causes developmental toxicity, induces fetal alcohol spectrum disorder (FASD) in humans, encompassing a whole range of developmental defects, including effects on neurodevelopment [206] In vitro, ACE in a concentration-dependent manner retarded embryonic growth by disrupting cell growth and differentiation in the WEC [220] Possible mechanisms of action behind ACE induced developmental toxicity include disrupted cell-cell interactions, oxidative stress, growth factor signaling disruption and altered gene expression [206]. Pathway analysis of ACE exposure in the ESTn shows signs of many of the proposed ACE mechanism of action being regulated, including the above stated disruption of genes involved in neural differentiation.

A comprehensive review of cohort studies examining the teratogenic effects of CBZ treatment during the first trimester in humans described a strong association between CBZ exposure and spina bifida [205]. Mechanisms of action of CBZ developmental toxicity remain poorly understood. In the present study, CBZ mainly regulated processes related to transcription and methylation, neural and blood vessel development and phosphorylation.

FLU exposure on gestational days 6-15 induced skeletal malformations and variations in mice, but no malformations related to neural development were reported [211]. However, in the WEC, delay in neuropore development and subtle effects on hindbrain segmentation, neural crest cell migration and cranial nerve abnormalities were detected after FLU exposure [221, 222]. FLU developmental toxicity may in part be explained through disruption of retinoic acid (RA) homeostasis. There is evidence that triazoles inhibit the catalytic activity of Cyp26 enzymes, which play an important role in RA degradation [223, 224]. Excess RA in the cell induces Cyp26a1 and Cyp26b1 gene expression in order to maintain RA homeostasis [225]. Changes in RA concentration may affect cell differentiation. FLU effects on Cyp26a1 and Cyp26b1 gene expression observed in the present study support this theory (Data not shown). Furthermore, in our study, FLU clearly induces gene nvolved in sterol- and cholesterol metabolic processes, being the triazole mode of action as antifungal agents. 
Although only a limited number of genes were regulated by MEHP, PENG and PHE, pathway gene distribution analysis did provide additional insight in possible developmental toxicity and mechanisms of action of these compounds. MEHP and PHE both downregulated a number of developmental related pathways. Furthermore, MEHP and PENG mainly induced pathways related to ion transport and oxidation, while PHE mainly inhibited a range of pathways involved in transcription, cell cycle, phosphorylation, ubiquitination and cell death.

The current knowledge on the diversity of in vivo mechanisms of action of the studied compounds is in line with the differences in gene expression profiles between compounds observed in the present study. It shows that the ESTn combined with transcriptomics can provide additional mechanistic information compared to the classical readout.

In this study, we additionally compared gene expression responses between a non-developmental toxic and a developmental toxic exposure concentration. This approach is in line with a recent proposal for validation of in vitro alternatives, in which not the compound per se but an "exposure", defined as a compound concentration or dose, is classified as a developmental toxicant, [101]. Our design was aimed at distinguishing between adaptive and adverse responses at the gene expression level. With the increased sensitivity of innovative molecular techniques for effect assessment, this distinction has gained importance in view of interpreting findings for risk assessment [226]. We observed that CBZ and FLU at the low concentrations enriched pathways involved in transcription and compound metabolism. Additionally, the low concentration CBZ enriched histone methylation related processes which represent a known pharmacological mechanism of action of CBZ [227]. Genes regulated only at the highest concentration CBZ or FLU perturbed a range of developmental, transcription and cellular related processes. In addition, the FLU high concentration resulted in enriched developmentally related genes as well as sterol metabolism-related genes, the triazole mechanism of action for antifungal activity [228]. Recently, a number of concentration response studies have been performed in multiple models, attempting to distinguish adaptive and adverse compound effects [229, 230]. For instance, in rat nasal cells, a low concentrations formaldehyde regulated p53 pathways, followed at increasing concentrations by regulation of pathways involved in cell cycle, DNA damage and apoptosis [229]. Furthermore, a study in the cardiac EST described FLU concentration dependent mechanisms of action at the transcriptome level [230]. Effects on processes related to development were observed at lower concentrations than those related to sterol metabolism, followed by enrichment of cell cycle gene expression at even higher concentrations. Taken together, current evidence suggests that up to a certain level of induction, general physiologica pathways such as related to transcription and metabolism may indicate adaptive responses, whereas the induction of developmental pathways may be more specifically indicating adverse responses. In addition, the magnitude of induction should be considered. More detailed studies will be needed to define the thresholds between adaptive and adverse responses in in vitro assays, in the interest of their interpretation in view of hazard and risk assessment.

\section{Lisexsers}




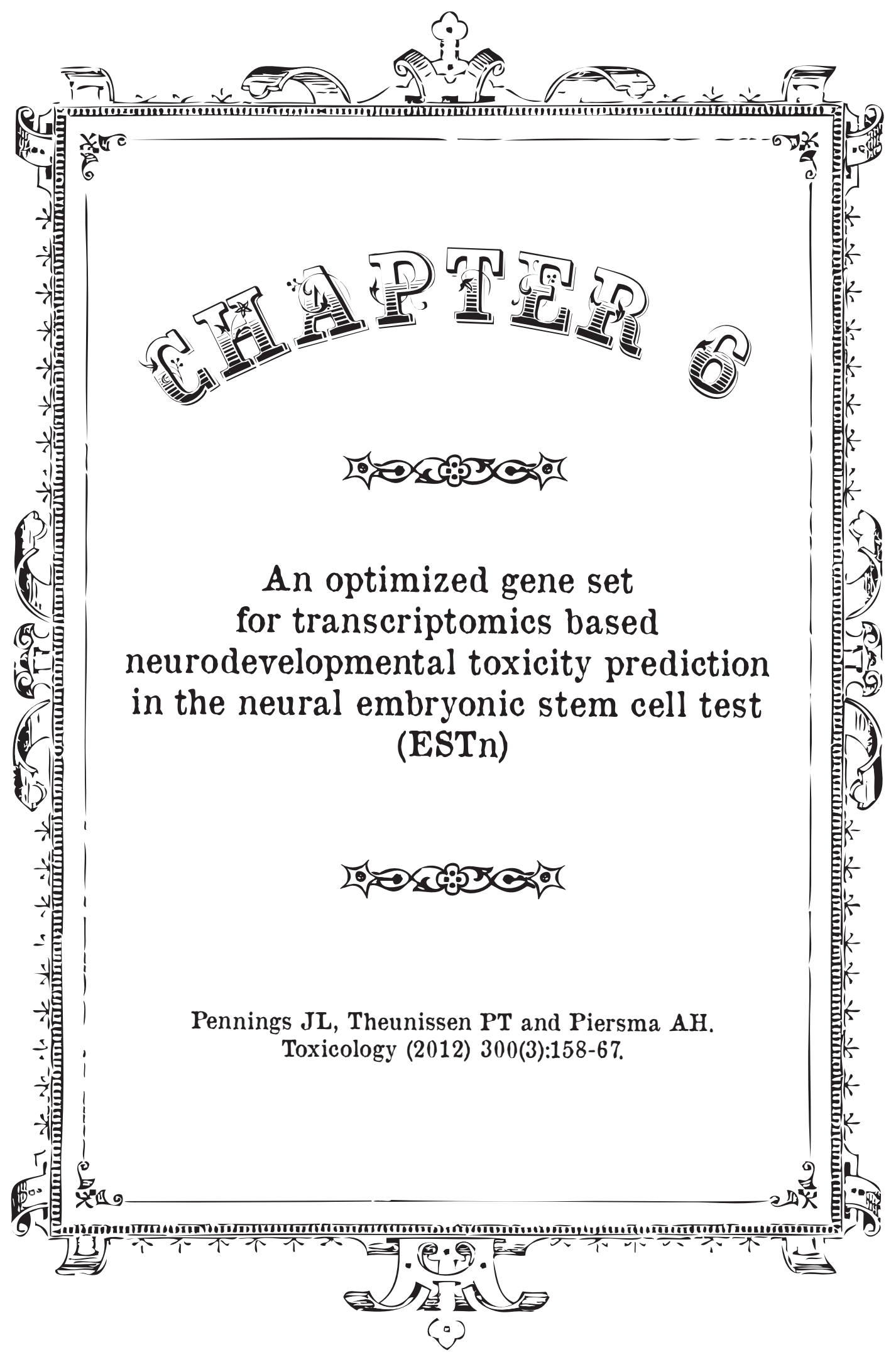




\section{Abstract}

T HE murine neural embryonic stem cell test (ESTn) is an in vitro model for neurodevelopmental toxicity testing. Recent studies have shown that application of transcriptomics analyses in the ESTn is useful for obtaining more accurate predictions as well as mechanistic insights. Gene expression responses due to stem cell neural differentiation versus toxicant exposure could be distinguished using the Principal Component Analysis based differentiation track algorithm. In this study, we performed a de novo analysis on combined raw data (10 compounds, 19 exposures) from three previous transcriptomics studies to identify an optimized gene set for neurodevelopmental toxicity prediction in the ESTn. By evaluating predictions of 200,000 randomly selected gene sets, we identified genes which significantly contributed to the prediction reliability. A set of 100 genes was obtained, predominantly involved in (neural) development. Further stringency restrictions resulted in a set of 29 genes that allowed for $84 \%$ prediction accuracy (area under the curve 94\%). We anticipate these gene sets will contribute to further improve ESTn transcriptomics studies aimed at compound risk assessment.

\section{Introduction}

T oxicological hazard identification is currently built upon animal tes 1 guidelines globally harmonized by the Organization for Economic Co-operation and Development (OECD). Under the new European chemica safety regulation (Regulation, Evaluation, and Authorization of Chemicals, REACH), reproductive and developmental toxicity studies are estimated to require approximately $60 \%$ of the number of animals needed for toxicity testing [12]. Therefore, increasing international research effort is put into developing alternative testing methods to predict reproductive and developmental toxicity, in order to allow for reduction, refinement and replacement of animal use. An in vitro developmental toxicity testing system validated by the European Centre for the Validation of Alternative Methods (ECVAM), is the embryonic stem cell test (EST) [17]. In the EST, compound developmental toxic effects on stem cel differentiation towards beating cardiomyocytes are determined. However, as the EST is primarily based on mesodermal-derived cardiomyocyte differentiation, it is likely less predictive for compounds that disturb ectodermal or endodermal tissue development [17]. Given that a common target for developmental toxicity is the developing nervous system, an assay based on neural differentiation of embryonic stem cells can be particularly valuable to further improve in vitro prediction accuracy for these compounds.

Building upon protocols described by Okabe et al. [52] and Bibel et al. [103], Theunissen et al. [142] designed a novel protocol for the neural embryonic stem cell test (ESTn). In this assay, mouse embryonic stem cells are stimulated towards multiple lineages of neural and other brain-associated cells by means of embryoid body formation, retinoic acid (RA) stimulation and serum deprivation. The potential of the ESTn in toxicity testing was demonstrated using the neurodevelopmental toxicant methylmercury chloride [142]. In subsequent studies performed in our lab, Theunissen et al. tested a range of compounds in the ESTn by means of microarray gene expression profiling [177, 203, 231]. These studies were motivated by the knowledge that molecular biological readou methods such as transcriptomics had already shown potential to improve the accuracy of the classic (cardiac) EST by providing more quantitative endpoints as well as providing mechanistic information [83, 232]. In these studies, also different time points [177] and compound concentrations [203, 231] were studied for both mechanistic comparisons between compound-induced responses as well as neurodevelopmental toxicity prediction.

The 'differentiation track algorithm' initially developed for classic EST transcriptomics studies $[83,233,234]$ was found to be applicable for toxicity prediction in the ESTn as well. This algorithm is based on (1) the finding that 
a Principal Component Analysis (PCA) applied to expression data for different time points during embryonic stem cell differentiation results in a visualization that represents a 'track' of their chronological order [234, 235] and (2) that compound-induced differentiation perturbation leads to deviation from this track [234]. An important requirement for algorithm prediction accuracy is to base the calculations on a set of genes which are regulated by normal (unexposed) differentiation and/or toxicity-related compound exposure. For classic EST transcriptomics, several such gene sets have been described [40, 83]. By means of an integrated analysis combining microarray and toxicological data from multiple studies, Pennings et al. assembled an optimized gene set for developmental toxicity prediction on classic EST transcriptomics data [84]. This set outperformed previously obtained gene sets, allowing for $83 \%$ overall accuracy with all misclassifications being attributable to ineffective data [84].

In the present study, a similar integrated analysis is applied to ESTn transcriptomics data from three previous studies [177, 203, 231]. By means of randomly sampling 200,000 gene sets and evaluating these in differentiation track algorithm toxicity prediction on 19 exposures using 10 compounds, genes significantly contributing to high prediction accuracy are determined. Following additional cross-validation, consistently high-scoring genes are combined into a novel gene set providing $84 \%$ accuracy for neurodevelopmental toxicity prediction.

\section{\&}

\section{Methods}

\section{Microarray samples and analysis}

Methods and data regarding ESTn culture conditions, compound exposures, assessment of differentiation inhibition, and microarray transcriptiona profiling have been described in full detail in previous publications from our group [142, 177, 203, 231]. Raw and normalized microarray data used in this study are available at ArrayExpress (www.ebi.ac.uk/arrayexpress/), under accession numbers E-TABM-1108, E-TABM-1205, and E-TABM-1216. As the three data sets used are not fully identical in their experimental design regarding time points and concentrations, some restrictions were applied to ensure mutual comparability. Unexposed controls at 0,24 , and $48 \mathrm{~h}$ after neural induction were included for each of the three studies; compound exposed samples at $24 \mathrm{~h}$ were included for the highest concentration tested in the ESTn (ID50 for neurodevelopmental toxicants, maximally feasible concentration without affecting cellular viability for non-developmenta toxicants) and, when available, the lowest exposure concentration tested (30 or
64 times below the high concentration) as a non-neurodevelopmentally toxic 'adaptive phase' control (no observed ESTn inhibition). This led to 218 out of 314 samples being selected for further analyses. An overview of selected compounds and concentrations is given in Table 1.

All statistical calculations used in this study were performed in R (www.r-project. org) [236] unless indicated otherwise. The algorithm for calculating p-values for differentiation track deviation has been described previously [83, 234]. Briefly, normalized gene expression data for a set of genes are log-transformed, and data for unexposed samples at $0 \mathrm{~h}, 24 \mathrm{~h}$, and $48 \mathrm{~h}$, together with compound treatment samples at $24 \mathrm{~h}$, are used in a PCA. Coordinates along the first and second principal component are calculated for each sample. P-values for deviation of the $24 \mathrm{~h}$ compound-exposed samples from the differentiation track are determined by applying a Hotelling T-test to these coordinates compared to those of the $24 \mathrm{~h}$ control cultures.

\section{Gene set optimization}

For the assembly of a predictive gene set for ESTn neural differentiation track deviation, we used a five-step approach that is largely analogous to an integrated analysis on EST cardiac differentiation transcriptomics data [84].

First, the data set was restricted to genes consistently regulated during (normal neural) differentiation in all three included data sets. For each study we selected the genes that showed significant differences in expression between the unexposed samples at $0 \mathrm{~h}, 24 \mathrm{~h}$, and $48 \mathrm{~h}$ (False Discovery Rate (FDR) $1 \%$, Absolute Fold Ratio (AFR) > 1.25), after which we determined the intersection between these lists. This first step reduced the number of genes in the data set from 20979 (all annotated genes on the microarray) to 4579.

Next, 200,000 randomly selected sets of 30 genes each were taken from the data set, so that each gene was sampled well over a 1000 times (average 1310 \pm 36 times). For each gene set, we calculated the p-value for differentiation track deviation for each $24 \mathrm{~h}$ compound exposed group, using the data of this group plus data of the matched 0,24 , and $48 \mathrm{~h}$ unexposed groups from the same study. Unexposed $24 \mathrm{~h}$ samples were used in the analysis as a time-matched reference group with (by definition) no differentiation track deviation. For each set, the calculated p-values for the various compound exposures were compared to their neurodevelopmental toxicity ( 1 for yes, 0 for no) by calculating the Spearman (rank-based) correlation. The lower p-values have more pronounced differentiation track deviation corresponding to neurodevelopmentally toxic exposures; therefore gene sets that have a strong negative correlation to the binary value for neurodevelopmental toxicity can be considered as most predictive. Genes in a gene set were scored as a hit if the 
correlation for a gene set was less than -0.60 , which corresponds to the top $5 \%$ of the obtained correlations.

In the third step, after the correlations for 200,000 gene sets were determined, it was determined how many times each gene was sampled and how many times it had scored a hit (i.e. was part of the top 5\% best predictive sets). This latter value was compared to the expected number of hits (i.e. $5 \%$ of the number of samplings). As for most of the genes this scoring ratio (actual hits predicted hits) followed a Gaussian distribution, this distribution was used to determine which genes had a significantly higher ratio, at an FDR of 5\%. Al identified genes were combined in a set (ESTn_enriched) and track deviation p-values were calculated using this set.

Fourth, a leave-one-out cross-validation (LOOCV) was carried out. This involved repeating the above procedure with each time one of the compound exposed groups left out and determining which genes were significantly enriched in the top $5 \%$ sets. These cross-validation gene sets were used to calculate the p-value for the left out group. Finally, those genes in the ESTn enriched set that were confirmed by all cross-validations were combined in a restricted gene set (ESTn_restricted), after which track deviation p-values were calculated using this set.

\section{Further gene set analyses}

The two obtained gene sets were evaluated for neurodevelopmental toxicity identification, using 19 compound/concentration exposure cultures from the data sets $[177,203,231]$ and the differentiation track algorithm. Compound/ concentration combinations were considered neurodevelopmentally toxic if they showed significant deviation from the differentiation track $($ at $\mathrm{p}<0.05)$ and as non-toxic otherwise. Additional accuracy evaluation was carried out by calculating the Area Under the Curve (AUC) for a Receiver Operating Characteristic (ROC) curve of p-values against the neurodevelopmental toxicity binary value.

Gene expression changes during normal (unexposed) differentiation or between compound exposures and their matching control were visualized in $\mathrm{R}$ (using averaged values per experimental condition)by means of PCA or by hierarchical clustering (Euclidean distance, Ward linkage).

Enrichment for Gene Ontology (GO) and other functional terms was determined using the DAVID Bioinformatics Resource website (http://david.abcc.ncifcrf. gov) [237] using default settings. Additionally, an in-house developed $\mathrm{R}$ application (based on the DAVID methodology) was used to determine enrichment for literature gene sets such as the MSigDB curated from genomics literature [238] and a stem cell development literature database described earlier [234] updated with additional more recent literature gene sets.
A network with gene, protein, and compound interactions was build in Cytoscape using data obtained from the STITCH database [239] (http://stitch. embl.de/). We used the gene symbols in the ESTn_enriched set as input, to le the STITCH search tool determine mutual interactions among the input genes as well as proteins or chemicals that the STITCH search tool finds associated with them. This query was performed twice, using both mouse and human as organism and otherwise default settings. The network description output files for mouse and human were merged into a single network description file to remove redundancy. This file was combined with gene information from Supplementary Table 2 for visualization in Cytoscape [240].

\section{\&̊ำ}

\section{Results}

\section{Gene set optimization and cross-validation}

After evaluating 200,000 randomly selected gene sets for the prediction of 19 exposure conditions, we found 100 genes to be significantly enriched among gene sets that show strong correlation between neurodevelopmental toxicity and differentiation track deviation. These genes were combined into a novel gene set which we name ESTn_enriched (Table 2). Using this gene set, predicted differentiation track showed $74 \%$ agreement with observed or literature-based neurodevelopmental toxicity at a p-value cutoff of 0.05 (Table 1). Othe prediction parameters support the predictive capacity of the gene set $(\mathrm{AUC}=$ $0.90, \mathrm{R}=-0.67)$.

Subsequent predictions using a leave-one-out cross-validation gave comparable p-values for most exposures as with the ESTn_enriched gene set. Overal accuracy here was $58 \%$, with an AUC of $70 \%$ and an R of -0.34 . Of the initial 100 genes, 92 were found to be significantly enriched in a majority, and 29 genes in all of the cross-validations. This latter, restricted, set was named ESTn_restricted (see upper part of Table 2) and largely corresponds to the genes in the former set that have the highest scoring ratios (Table 2, Figure 1) Predictions using the ESTn_restricted gene set showed an improved accuracy compared to those based on the ESTn_enriched set, as the accuracy was $84 \%$, the AUC was 0.94 and the correlation was -0.74 .

\section{Biological characterization}

PCA on ESTn_enriched set gene expression for the $0 \mathrm{~h}, 24 \mathrm{~h}$, and $48 \mathrm{~h}$ controls, together with the various $24 \mathrm{~h}$ compound exposures, shows the differentiation track for normal (unexposed) differentiation (Figure 2). Compound exposures 
Table 1 Compound exposures and corresponding $\mathrm{p}$-values obtained using the different gene sets.

\begin{tabular}{|c|c|c|c|c|c|c|c|}
\hline $\begin{array}{l}\bar{\Xi} \\
\stackrel{\Xi}{\Xi}\end{array}$ & $\begin{array}{l}\overrightarrow{\vec{E}} \\
\vec{E}\end{array}$ & 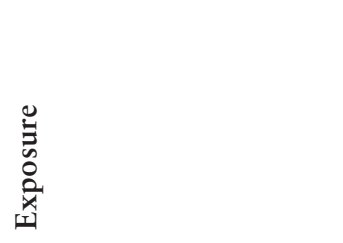 & 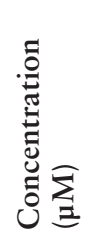 & 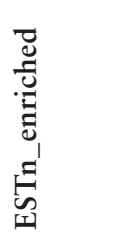 & 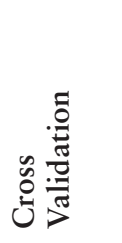 & 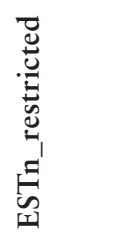 & 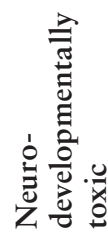 \\
\hline mehgH & 1 & Methylmercury chloride & 0.025 & 0.045 & $0.086 *$ & 0.009 & yes \\
\hline cypL & 2 & Cyproconazole & 1.5 & 0.300 & 0.154 & 0.280 & no \\
\hline сурН & 2 & Cyproconazole & 100 & $0.379 *$ & $0.624 *$ & $0.160 *$ & yes \\
\hline hexL & 2 & Hexaconazole & 0.4 & 0.472 & 0.530 & 0.548 & no \\
\hline hexH & 2 & Hexaconazole & 25 & 0.454 & $0.004 *$ & 0.587 & no \\
\hline vpaL & 2 & Valproic acid & 15 & 0.442 & 0.282 & 0.305 & no \\
\hline vpaH & 2 & Valproic acid & 1000 & $<0.001$ & $<0.001$ & $<0.001$ & yes \\
\hline aceL & 3 & Acetaldehyde & 1 & 0.221 & 0.275 & 0.361 & no \\
\hline aceH & 3 & Acetaldehyde & 30 & 0.006 & 0.009 & 0.009 & yes \\
\hline cbzL & 3 & Carbamazepine & 3 & 0.746 & 0.707 & 0.815 & no \\
\hline $\mathrm{cbzH}$ & 3 & Carbamazepine & 100 & $0.076 *$ & $0.258 *$ & 0.046 & yes \\
\hline fluL & 3 & Flusilazole & 0.3 & 0.544 & $0.017 *$ & 0.378 & no \\
\hline fluH & 3 & Flusilazole & 10 & 0.002 & 0.002 & 0.002 & yes \\
\hline mehpL & 3 & Monoethylhexylphtalate & 3 & 0.575 & 0.522 & 0.388 & no \\
\hline mehpH & 3 & Monoethylhexylphtalate & 1000 & 0.006 & 0.019 & $<0.001$ & yes \\
\hline pengL & 3 & Penicillin G & 30 & 0.554 & 0.502 & 0.568 & no \\
\hline pengH & 3 & Penicillin G & 1000 & $0.036 *$ & $0.018 *$ & $0.035 *$ & no \\
\hline pheL & 3 & Phenytoin & 1 & $0.024 *$ & $0.016 *$ & $0.020 *$ & no \\
\hline pheH & 3 & Phenytoin & 30 & $0.055 *$ & $0.228 *$ & 0.045 & yes \\
\hline correct & & & & 14 & 11 & 16 & \\
\hline$\%$ & & & & $73.7 \%$ & $57.9 \%$ & $84.2 \%$ & \\
\hline $\mathrm{R}$ & & & & -0.672 & -0.336 & -0.743 & \\
\hline AUC & & & & 0.896 & 0.698 & 0.938 & \\
\hline
\end{tabular}

Incorrect classifications are marked with an asterisk are positioned mostly close to the unexposed $24 \mathrm{~h}$ control for the non-toxic exposures but deviate further from the track for toxic exposures (Figure 2).

Visualization of compound-induced gene expression changes as compared to time-matched unexposed controls shows that, in general, higher compound exposure levels result in a stronger gene expression response (Figure 3).

Functional enrichment analysis on the ESTn_enriched in DAVID showed significant enrichment for a number of GO terms related to neural development such as "neuron differentiation", "neurogenesis", "neural crest cell development", "axonogenesis", and "neuron recognition"; which together covered 18 genes in the set. Additionally, several other development related terms were enriched, such as "anterior/posterior pattern formation", "embryonic morphogenesis", "embryonic organ development", "cell morphogenesis", and "Wnt-signaling pathway" which, when combined, together comprise 30 genes. Also functional terms involving homeobox genes or domains were enriched (12 genes), as was the case for transcription ( 25 genes). These functional associations are summarized in Table 2.

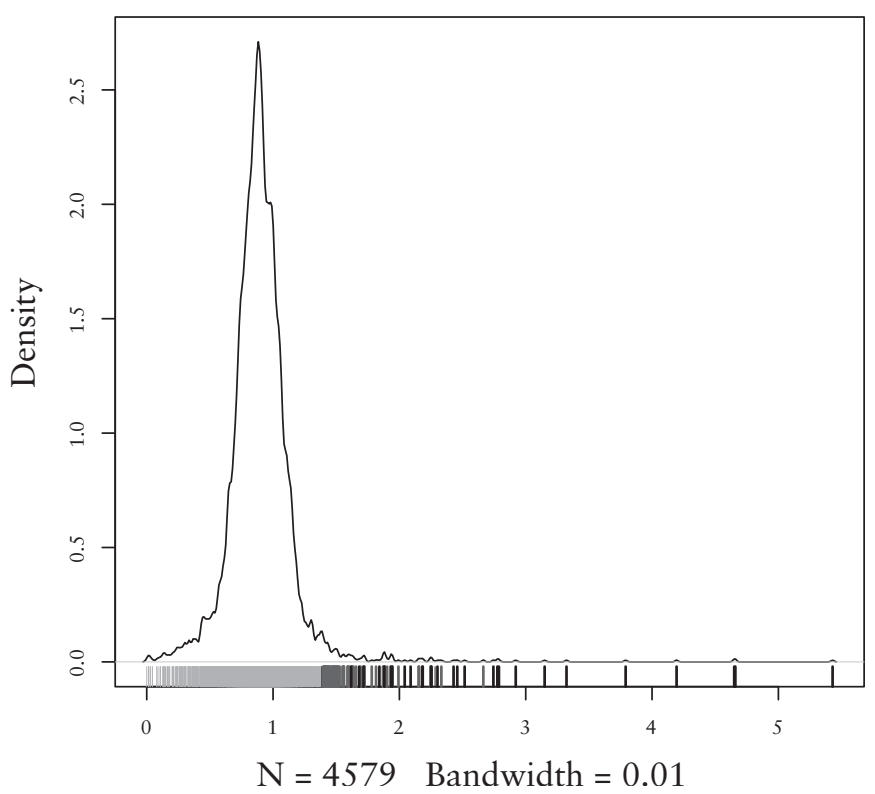

Figure 1 Density plot for scoring ratios. Vertical ticks below the distribution curve ndicate significance status of individual genes. Black, not significant; green, significantly enriched (FDR 5\%); red, significant and also part of the ESTn_restricted set. 
PCA using ESTn_enriched gene set

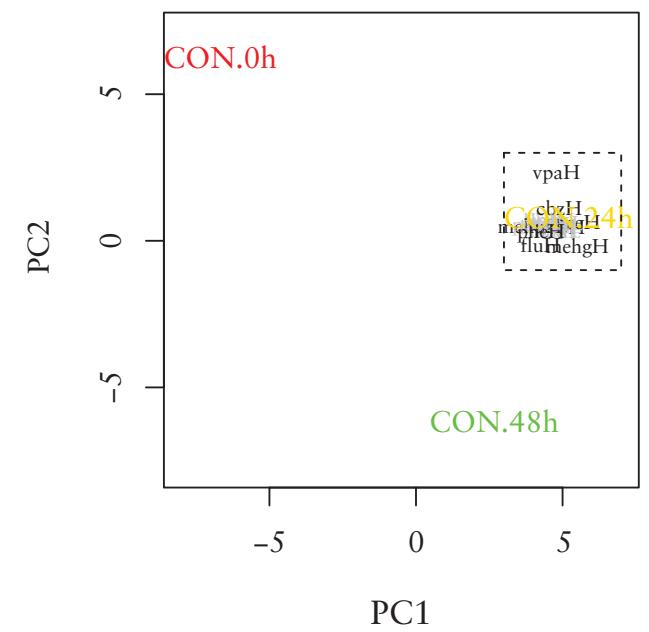

B

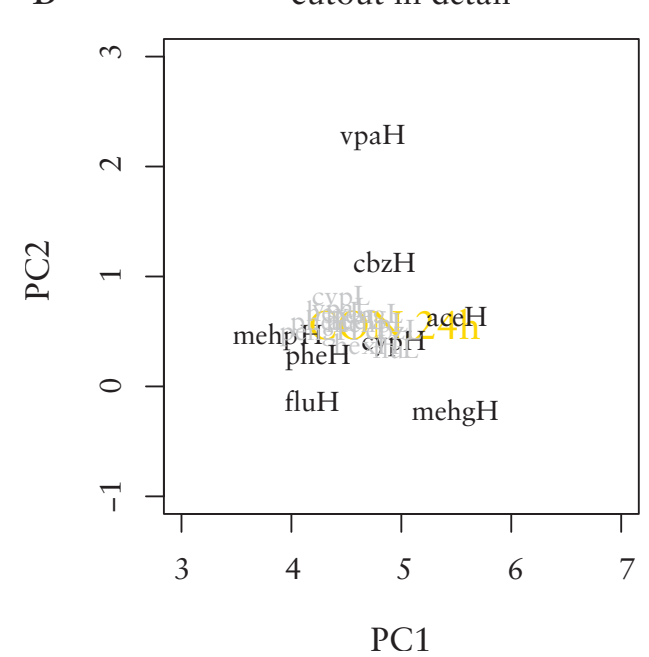

Figure 2 Track visualization based on the ESTn_enriched gene set. (A) PCA for all experimental conditions (red, $0 \mathrm{~h}$ control; yellow, 24h control; green, 48h control; black, 24h toxic exposure; grey, 24h non-toxic exposure) (B) dashed cutout in detail.

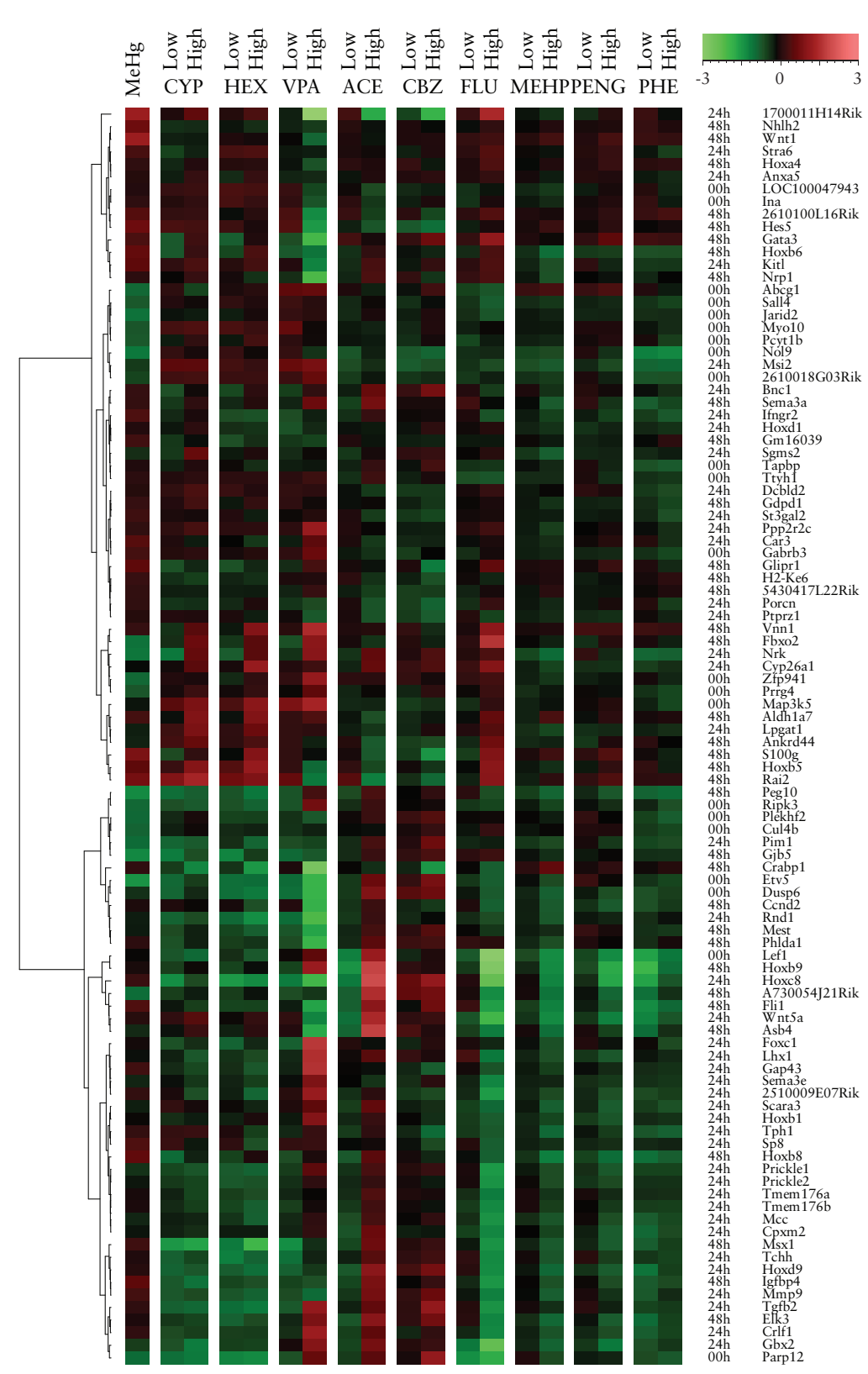

Figure 3 Expression response to compound exposure for genes in the ESTn_enriched set. Peak expression time points are indicated along with gene symbols. 
Table 2 Genes in the ESTn_enriched set

\begin{tabular}{|c|c|c|c|c|c|c|c|c|c|c|c|c|}
\hline 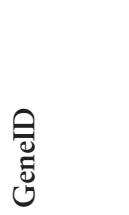 & 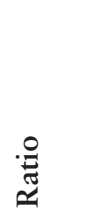 & 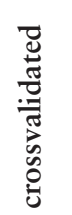 & $\begin{array}{l}\overline{0} \\
\text { है } \\
\text { हn }\end{array}$ & 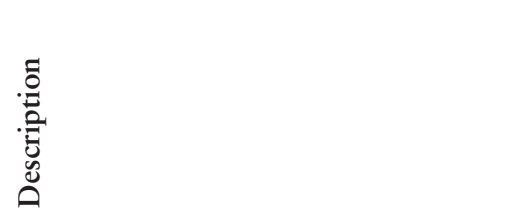 & 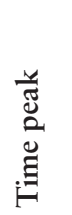 & 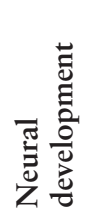 & 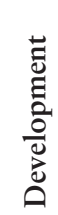 & 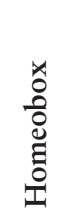 & 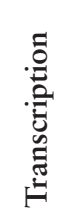 & 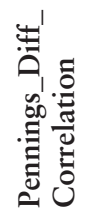 & 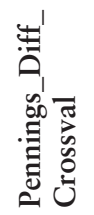 & 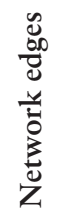 \\
\hline 16842 & 2,502 & yes & Lef1 & lymphoid enhancer binding factor 1 & $\mathrm{Oh}$ & 0 & 1 & 0 & 1 & 1 & 0 & 11 \\
\hline 67082 & 5,173 & yes & 1700011H14Rik & RIKEN cDNA 1700011H14 gene & $24 \mathrm{~h}$ & 0 & 0 & 0 & 0 & 0 & 0 & 0 \\
\hline 72190 & 2,426 & yes & 2510009E07Rik & RIKEN cDNA 2510009E07 gene & $24 \mathrm{~h}$ & 0 & 0 & 0 & 0 & 1 & 0 & 0 \\
\hline 12173 & 1,797 & yes & Bnc1 & basonuclin 1 & $24 \mathrm{~h}$ & 0 & 0 & 0 & 1 & 0 & 0 & 0 \\
\hline 14432 & 2,045 & yes & Gap43 & growth associated protein 43 & $24 \mathrm{~h}$ & 1 & 1 & 0 & 0 & 0 & 0 & 3 \\
\hline 15429 & 2,145 & yes & Hoxd1 & homeobox D1 & $24 \mathrm{~h}$ & 0 & 1 & 1 & 1 & 1 & 1 & 1 \\
\hline 17311 & 2,088 & yes & Kitl & kit ligand & $24 \mathrm{~h}$ & 1 & 0 & 0 & 0 & 0 & 0 & 12 \\
\hline 16869 & 2,555 & yes & Lhx1 & LIM homeobox protein 1 & $24 \mathrm{~h}$ & 1 & 1 & 1 & 1 & 1 & 1 & 3 \\
\hline 27206 & 5,163 & yes & Nrk & Nik related kinase & $24 \mathrm{~h}$ & 0 & 0 & 0 & 0 & 0 & 0 & 0 \\
\hline 18712 & 2,422 & yes & Pim1 & proviral integration site 1 & $24 \mathrm{~h}$ & 0 & 0 & 0 & 0 & 0 & 0 & 2 \\
\hline 269643 & 1,905 & yes & Ppp2r2c & $\begin{array}{l}\text { protein phosphatase } 2 \text {, regulatory } \\
\text { subunit B, gamma isoform }\end{array}$ & $24 \mathrm{~h}$ & 0 & 0 & 0 & 0 & 0 & 0 & 2 \\
\hline 106042 & 2,500 & yes & Prickle1 & prickle homolog 1 (Drosophila) & $24 \mathrm{~h}$ & 0 & 0 & 0 & 0 & 0 & 0 & 3 \\
\hline 219151 & 2,794 & yes & Scara3 & scavenger receptor class $\mathrm{A}$, member 3 & $24 \mathrm{~h}$ & 0 & 0 & 0 & 0 & 1 & 1 & 0 \\
\hline 20349 & 3,095 & yes & Sema3e & semaphorin $3 \mathrm{E}$ & $24 \mathrm{~h}$ & 1 & 1 & 0 & 0 & 0 & 0 & 2 \\
\hline 21808 & 3,047 & yes & Tgfb2 & transforming growth factor, beta 2 & $24 \mathrm{~h}$ & 1 & 1 & 0 & 0 & 1 & 1 & 8 \\
\hline 21990 & 4,209 & yes & Tph1 & tryptophan hydroxylase 1 & $24 \mathrm{~h}$ & 0 & 0 & 0 & 0 & 1 & 0 & 3 \\
\hline 22418 & 3,689 & yes & Wnt5a & $\begin{array}{l}\text { wingless-related MMTV integration } \\
\text { site } 5 \mathrm{~A}\end{array}$ & $24 \mathrm{~h}$ & 0 & 1 & 0 & 0 & 0 & 0 & 12 \\
\hline 70441 & 3,243 & yes & 2610100L16Rik & RIKEN cDNA 2610100L16 gene & $48 \mathrm{~h}$ & 0 & 0 & 0 & 0 & 0 & 0 & 0 \\
\hline 26358 & 2,697 & yes & Aldh1a7 & $\begin{array}{l}\text { aldehyde dehydrogenase family } 1 \text {, } \\
\text { subfamily A7 }\end{array}$ & $48 \mathrm{~h}$ & 0 & 0 & 0 & 0 & 0 & 0 & 2 \\
\hline 12903 & 4,656 & yes & Crabp1 & cellular retinoic acid binding protein I & $48 \mathrm{~h}$ & 0 & 0 & 0 & 0 & 0 & 0 & 6 \\
\hline 14247 & 3,497 & yes & Fli1 & Friend leukemia integration 1 & $48 \mathrm{~h}$ & 0 & 0 & 0 & 1 & 0 & 0 & 1 \\
\hline 14462 & 2,158 & yes & Gata3 & GATA binding protein 3 & $48 \mathrm{~h}$ & 0 & 0 & 0 & 1 & 0 & 0 & 5 \\
\hline 15413 & 3,081 & yes & Hoxb5 & homeobox B5 & $48 \mathrm{~h}$ & 0 & 1 & 1 & 1 & 1 & 0 & 1 \\
\hline 15414 & 6,027 & yes & Hoxb6 & homeobox B6 & $48 \mathrm{~h}$ & 0 & 1 & 1 & 1 & 1 & 0 & 1 \\
\hline 16010 & 2,267 & yes & Igfbp4 & $\begin{array}{l}\text { insulin-like growth factor binding } \\
\text { protein } 4\end{array}$ & $48 \mathrm{~h}$ & 0 & 0 & 0 & 0 & 0 & 0 & 3 \\
\hline 17294 & 2,145 & yes & Mest & mesoderm specific transcript & $48 \mathrm{~h}$ & 0 & 0 & 0 & 0 & 0 & 0 & 1 \\
\hline 18186 & 2,319 & yes & Nrp1 & neuropilin 1 & $48 \mathrm{~h}$ & 1 & 1 & 0 & 0 & 1 & 0 & 8 \\
\hline 170676 & 2,729 & yes & Peg10 & paternally expressed 10 & $48 \mathrm{~h}$ & 0 & 0 & 0 & 0 & 0 & 0 & 0 \\
\hline
\end{tabular}


Table 2 Continued

\begin{tabular}{|c|c|c|c|c|c|c|c|c|c|c|c|c|}
\hline 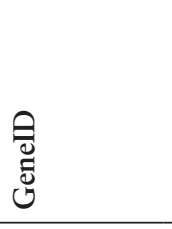 & 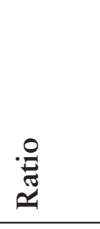 & 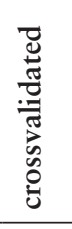 & $\begin{array}{l}\overline{0} \\
\text { हे } \\
\text { 心 }\end{array}$ & 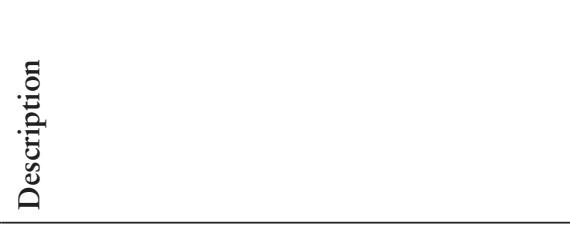 & 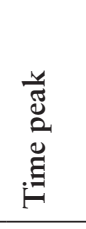 & 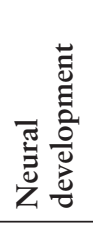 & 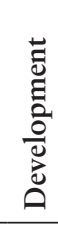 & 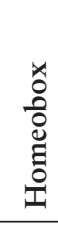 & 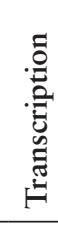 & 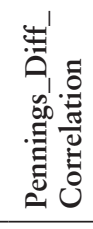 & 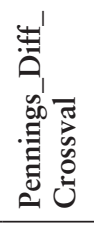 & 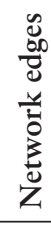 \\
\hline 22408 & 1,867 & yes & Wnt1 & wingless-related MMTV integration site 1 & $48 \mathrm{~h}$ & 1 & 1 & 0 & 0 & 0 & 0 & 12 \\
\hline 70415 & 1,677 & no & 2610018G03Rik & RIKEN cDNA 2610018G03 gene & oh & 0 & 0 & 0 & 0 & 0 & 0 & 0 \\
\hline 11307 & 1,597 & no & Abcg1 & $\begin{array}{l}\text { ATP-binding cassette, sub-family G } \\
\text { (WHITE), member } 1\end{array}$ & oh & 0 & 0 & 0 & 0 & 0 & 0 & 2 \\
\hline 72584 & 1,676 & no & Cul4b & cullin 4B & Oh & 0 & 0 & 0 & 0 & 0 & 0 & 1 \\
\hline 67603 & 1,642 & no & Dusp6 & dual specificity phosphatase 6 & $0 \mathrm{~h}$ & 0 & 0 & 0 & 0 & 0 & 0 & 3 \\
\hline 104156 & 1,694 & no & Etv5 & ets variant gene 5 & Oh & 0 & 0 & 0 & 1 & 0 & 0 & 3 \\
\hline 14402 & 2,085 & no & Gabrb3 & $\begin{array}{l}\text { gamma-aminobutyric acid (GABA) } \\
\text { A receptor, subunit beta } 3\end{array}$ & $0 \mathrm{~h}$ & 1 & 0 & 0 & 0 & 0 & 0 & 1 \\
\hline 226180 & 1,575 & no & Ina & $\begin{array}{l}\text { internexin neuronal intermediate filament } \\
\text { protein, alpha }\end{array}$ & $0 \mathrm{~h}$ & 1 & 1 & 0 & 0 & 0 & 0 & 0 \\
\hline 16468 & 1,664 & no & Jarid2 & jumonji, AT rich interactive domain 2 & $0 \mathrm{~h}$ & 0 & 1 & 0 & 1 & 0 & 0 & 1 \\
\hline 100047943 & 1,906 & no & LOC100047943 & similar to Ina protein & $0 \mathrm{~h}$ & 0 & 0 & 0 & 0 & 0 & 0 & 0 \\
\hline 26408 & 1,771 & no & Map3k5 & $\begin{array}{l}\text { mitogen-activated protein kinase kinase } \\
\text { kinase } 5\end{array}$ & oh & 0 & 0 & 0 & 0 & 0 & 0 & 1 \\
\hline 17909 & 1,785 & no & Myo10 & myosin $\mathrm{X}$ & Oh & 0 & 0 & 0 & 0 & 0 & 0 & 0 \\
\hline 74035 & 1,732 & no & Nol9 & nucleolar protein 9 & Oh & 0 & 0 & 0 & 0 & 0 & 0 & 0 \\
\hline 243771 & 1,915 & no & Parp12 & $\begin{array}{l}\text { poly (ADP-ribose) polymerase family, } \\
\text { member } 12\end{array}$ & Oh & 0 & 0 & 0 & 0 & 0 & 0 & 1 \\
\hline 236899 & 1,911 & no & Pcyt1b & $\begin{array}{l}\text { phosphate cytidylyltransferase } 1, \\
\text { choline, beta isoform }\end{array}$ & $0 \mathrm{~h}$ & 0 & 0 & 0 & 0 & 0 & 0 & 1 \\
\hline 71801 & 2,076 & no & Plekhf2 & $\begin{array}{l}\text { pleckstrin homology domain containing, } \\
\text { family F member } 2\end{array}$ & Oh & 0 & 0 & 0 & 0 & 0 & 0 & 0 \\
\hline 228413 & 1,556 & no & $\operatorname{Prrg} 4$ & $\begin{array}{l}\text { proline rich Gla (G-carboxyglutamic } \\
\text { acid) } 4 \text { (transmembrane) }\end{array}$ & Oh & 0 & 0 & 0 & 0 & 0 & 0 & 1 \\
\hline 56532 & 1,648 & no & Ripk3 & $\begin{array}{l}\text { receptor-interacting serine-threonine } \\
\text { kinase } 3\end{array}$ & Oh & 0 & 0 & 0 & 0 & 0 & 0 & 1 \\
\hline 99377 & 2,150 & no & Sall4 & sal-like 4 (Drosophila) & $0 \mathrm{~h}$ & 0 & 1 & 0 & 1 & 0 & 0 & 5 \\
\hline 21356 & 1,637 & no & Tapbp & TAP binding protein & $0 \mathrm{~h}$ & 0 & 0 & 0 & 0 & 0 & 0 & 0 \\
\hline 57776 & 1,587 & no & Ttyh1 & tweety homolog 1 (Drosophila) & Oh & 1 & 0 & 0 & 0 & 0 & 0 & 0 \\
\hline 407812 & 1,567 & no & Zfp941 & zinc finger protein 941 & Oh & 0 & 0 & 0 & 1 & 0 & 0 & 0 \\
\hline 11747 & 1,570 & no & Anxa5 & annexin A5 & $24 \mathrm{~h}$ & 0 & 0 & 0 & 0 & 0 & 0 & 4 \\
\hline 12350 & 1,880 & no & Car3 & carbonic anhydrase 3 & $24 \mathrm{~h}$ & 0 & 0 & 0 & 0 & 0 & 0 & 0 \\
\hline
\end{tabular}


Table 2 Continued

\begin{tabular}{|c|c|c|c|c|c|c|c|c|c|c|c|c|}
\hline 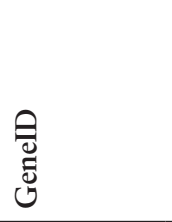 & 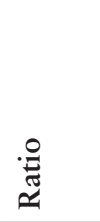 & 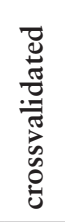 & $\begin{array}{l}\overline{0} \\
\text { हो } \\
\text { के }\end{array}$ & 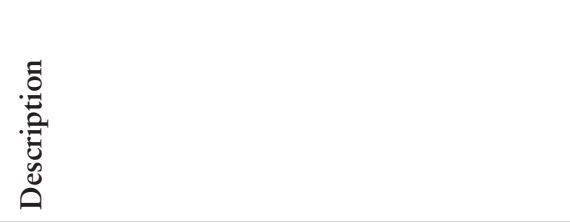 & 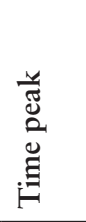 & 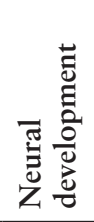 & 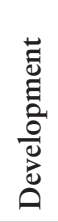 & 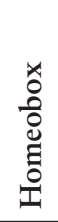 & 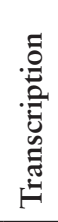 & 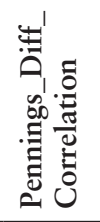 & 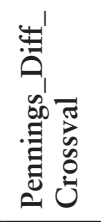 & 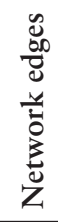 \\
\hline 55987 & 1,691 & no & Cpxm2 & carboxypeptidase X 2 (M14 family) & $24 \mathrm{~h}$ & 0 & 0 & 0 & 0 & 0 & 0 & 0 \\
\hline 12931 & 1,806 & no & Crlf1 & cytokine receptor-like factor 1 & $24 \mathrm{~h}$ & 0 & 0 & 0 & 0 & 0 & 0 & 0 \\
\hline 13082 & 1,977 & no & Сур26a1 & $\begin{array}{l}\text { cytochrome P450, family } 26 \text {, subfamily a, } \\
\text { polypeptide } 1\end{array}$ & $24 \mathrm{~h}$ & 1 & 1 & 0 & 0 & 0 & 0 & 7 \\
\hline 73379 & 1,561 & no & Dcbld2 & $\begin{array}{l}\text { discoidin, CUB and LCCL domain } \\
\text { containing } 2\end{array}$ & $24 \mathrm{~h}$ & 0 & 0 & 0 & 0 & 0 & 0 & 1 \\
\hline 17300 & 2,159 & no & Foxc1 & forkhead box C1 & $24 \mathrm{~h}$ & 1 & 1 & 0 & 1 & 1 & 1 & 4 \\
\hline 14472 & 2,493 & no & Gbx2 & gastrulation brain homeobox 2 & $24 \mathrm{~h}$ & 1 & 1 & 1 & 1 & 1 & 1 & 3 \\
\hline 15407 & 1,674 & no & Hoxb1 & homeobox B1 & $24 \mathrm{~h}$ & 0 & 1 & 1 & 1 & 1 & 1 & 6 \\
\hline 15426 & 2,212 & no & Hoxc8 & homeobox C8 & $24 \mathrm{~h}$ & 1 & 1 & 1 & 1 & 0 & 0 & 2 \\
\hline 15438 & 1,725 & no & Hoxd9 & homeobox D9 & $24 \mathrm{~h}$ & 1 & 1 & 1 & 1 & 1 & 0 & 1 \\
\hline 15980 & 2,394 & no & Ifngr2 & interferon gamma receptor 2 & $24 \mathrm{~h}$ & 0 & 0 & 0 & 0 & 0 & 0 & 1 \\
\hline 226856 & 1,732 & no & Lpgat1 & $\begin{array}{l}\text { lysophosphatidylglycerol } \\
\text { acyltransferase } 1\end{array}$ & $24 \mathrm{~h}$ & 0 & 0 & 0 & 0 & 0 & 0 & 1 \\
\hline 328949 & 2,089 & no & Mcc & mutated in colorectal cancers & $24 \mathrm{~h}$ & 0 & 0 & 0 & 0 & 0 & 0 & 0 \\
\hline 17395 & 2,389 & no & Mmp9 & matrix metallopeptidase 9 & $24 \mathrm{~h}$ & 0 & 1 & 0 & 0 & 0 & 0 & 9 \\
\hline 76626 & 2,093 & no & Msi2 & Musashi homolog 2 (Drosophila) & $24 \mathrm{~h}$ & 0 & 0 & 0 & 0 & 0 & 0 & 0 \\
\hline 53627 & 2,589 & no & Porcn & porcupine homolog (Drosophila) & $24 \mathrm{~h}$ & 0 & 0 & 0 & 0 & 0 & 0 & 2 \\
\hline 243548 & 1,558 & no & Prickle2 & prickle homolog 2 (Drosophila) & $24 \mathrm{~h}$ & 0 & 0 & 0 & 0 & 0 & 0 & 2 \\
\hline 19283 & 1,643 & no & Ptprz1 & $\begin{array}{l}\text { protein tyrosine phosphatase, receptor } \\
\text { type } Z \text {, polypeptide } 1\end{array}$ & $24 \mathrm{~h}$ & 1 & 0 & 0 & 0 & 1 & 0 & 2 \\
\hline 223881 & 1,731 & no & Rnd1 & Rho family GTPase 1 & $24 \mathrm{~h}$ & 0 & 0 & 0 & 0 & 0 & 0 & 4 \\
\hline 74442 & 1,655 & no & Sgms2 & sphingomyelin synthase 2 & $24 \mathrm{~h}$ & 0 & 0 & 0 & 0 & 0 & 0 & 0 \\
\hline 320145 & 1,601 & no & Sp8 & trans-acting transcription factor 8 & $24 \mathrm{~h}$ & 0 & 1 & 0 & 0 & 0 & 0 & 0 \\
\hline 20444 & 1,551 & no & St3gal2 & $\begin{array}{l}\text { ST3 beta-galactoside alpha-2,3- } \\
\text { sialyltransferase } 2\end{array}$ & $24 \mathrm{~h}$ & 0 & 0 & 0 & 0 & 0 & 0 & 0 \\
\hline 20897 & 1,840 & no & Stra6 & stimulated by retinoic acid gene 6 & $24 \mathrm{~h}$ & 0 & 0 & 0 & 0 & 0 & 0 & 4 \\
\hline 99681 & 2,015 & no & Tchh & trichohyalin & $24 \mathrm{~h}$ & 0 & 0 & 0 & 0 & 0 & 0 & 0 \\
\hline 66058 & 1,587 & no & Tmem176a & transmembrane protein $176 \mathrm{~A}$ & $24 \mathrm{~h}$ & 0 & 0 & 0 & 0 & 0 & 0 & 1 \\
\hline 65963 & 1,593 & no & Tmem176b & transmembrane protein $176 \mathrm{~B}$ & $24 \mathrm{~h}$ & 0 & 0 & 0 & 0 & 0 & 0 & 1 \\
\hline 100043272 & 1,809 & no & 5430417L22Rik & RIKEN cDNA 5430417L22 gene & $48 \mathrm{~h}$ & 0 & 0 & 0 & 0 & 0 & 0 & 0 \\
\hline 320862 & 1,622 & no & A730054J21Rik & RIKEN cDNA A730054J21 gene & $48 \mathrm{~h}$ & 0 & 0 & 0 & 0 & 1 & 0 & 0 \\
\hline
\end{tabular}


Table 2 Continued

\begin{tabular}{|c|c|c|c|c|c|c|c|c|c|c|c|c|}
\hline $\begin{array}{l}\text { 总 } \\
\text { ज̃ }\end{array}$ & 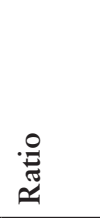 & 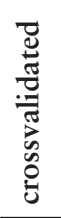 & $\begin{array}{l}\overline{0} \\
\text { हे } \\
\text { हn }\end{array}$ & 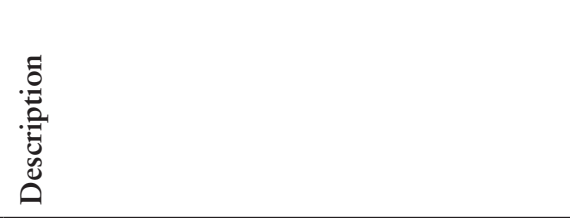 & 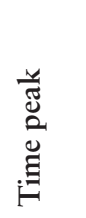 & 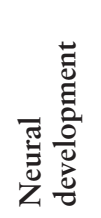 & 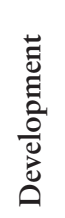 & 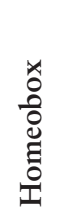 & 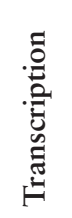 & 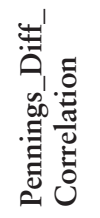 & 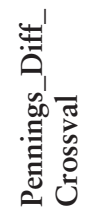 & 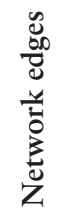 \\
\hline 329154 & 1,600 & no & Ankrd44 & ankyrin repeat domain 44 & $48 \mathrm{~h}$ & 0 & 0 & 0 & 0 & 0 & 0 & 0 \\
\hline 65255 & 2,085 & no & Asb4 & $\begin{array}{l}\text { ankyrin repeat and SOCS box- } \\
\text { containing } 4\end{array}$ & $48 \mathrm{~h}$ & 0 & 0 & 0 & 0 & 1 & 1 & 1 \\
\hline 12444 & 1,818 & no & Ccnd2 & cyclin D2 & $48 \mathrm{~h}$ & 0 & 0 & 0 & 0 & 0 & 0 & 9 \\
\hline 13713 & 1,772 & no & Elk3 & ELK3, member of ETS oncogene family & $48 \mathrm{~h}$ & 0 & 0 & 0 & 1 & 0 & 0 & 3 \\
\hline 230904 & 1,592 & no & Fbxo2 & F-box protein 2 & $48 \mathrm{~h}$ & 0 & 0 & 0 & 0 & 0 & 0 & 1 \\
\hline 66569 & 1,667 & no & Gdpd1 & $\begin{array}{l}\text { glycerophosphodiester phosphodiesterase } \\
\text { domain containing } 1\end{array}$ & $48 \mathrm{~h}$ & 0 & 0 & 0 & 0 & 0 & 0 & 0 \\
\hline 14622 & 1,585 & no & Gjb5 & gap junction protein, beta 5 & $48 \mathrm{~h}$ & 0 & 1 & 0 & 0 & 0 & 0 & 0 \\
\hline 73690 & 1,592 & no & Glipr1 & GLI pathogenesis-related 1 (glioma) & $48 \mathrm{~h}$ & 0 & 0 & 0 & 0 & 0 & 0 & 1 \\
\hline 100036521 & 1,630 & no & Gm16039 & predicted gene 16039 & $48 \mathrm{~h}$ & 0 & 0 & 0 & 0 & 0 & 0 & 0 \\
\hline 14979 & 1,621 & no & H2-Ke6 & H2-K region expressed gene 6 & $48 \mathrm{~h}$ & 0 & 0 & 0 & 1 & 0 & 0 & 0 \\
\hline 15208 & 1,606 & no & Hes5 & $\begin{array}{l}\text { hairy and enhancer of split } 5 \\
\text { (Drosophila) }\end{array}$ & $48 \mathrm{~h}$ & 1 & 1 & 0 & 1 & 0 & 0 & 2 \\
\hline 15401 & 1,768 & no & Hoxa4 & homeobox A4 & $48 \mathrm{~h}$ & 0 & 1 & 1 & 1 & 0 & 0 & 1 \\
\hline 15416 & 2,958 & no & Hoxb8 & homeobox B8 & $48 \mathrm{~h}$ & 0 & 1 & 1 & 1 & 0 & 0 & 2 \\
\hline 15417 & 1,586 & no & Hoxb9 & homeobox B9 & $48 \mathrm{~h}$ & 0 & 1 & 1 & 1 & 1 & 0 & 2 \\
\hline 17701 & 1,764 & no & Msx1 & homeobox, msh-like 1 & $48 \mathrm{~h}$ & 0 & 1 & 1 & 1 & 0 & 0 & 11 \\
\hline 18072 & 1,669 & no & Nhlh2 & nescient helix loop helix 2 & $48 \mathrm{~h}$ & 0 & 1 & 0 & 1 & 0 & 0 & 0 \\
\hline 21664 & 1,563 & no & Phlda1 & $\begin{array}{l}\text { pleckstrin homology-like domain, } \\
\text { family A, member } 1\end{array}$ & $48 \mathrm{~h}$ & 0 & 0 & 0 & 0 & 1 & 0 & 2 \\
\hline 24004 & 1,721 & no & Rai2 & retinoic acid induced 2 & $48 \mathrm{~h}$ & 0 & 0 & 0 & 0 & 0 & 0 & 1 \\
\hline 12309 & 1,656 & no & S100g & S100 calcium binding protein $G$ & $48 \mathrm{~h}$ & 0 & 0 & 0 & 0 & 0 & 0 & 0 \\
\hline 20346 & 1,573 & no & Sema3a & semaphorin $3 \mathrm{~A}$ & $48 \mathrm{~h}$ & 1 & 1 & 0 & 0 & 0 & 0 & 7 \\
\hline 22361 & 2,113 & no & Vnn1 & $\operatorname{vanin} 1$ & $48 \mathrm{~h}$ & 0 & 0 & 0 & 0 & 0 & 0 & 1 \\
\hline
\end{tabular}

Association with neural or stem cell development was also found among the most significantly enriched gene sets from literature gene set databases. The most significant among these were the "Pennings_Diff_Correlation" (19 genes) and "Pennings_Diff_crossval" (8 genes) sets from a previous gene set optimization on cardiac EST transcriptomics data [84]. Various other gene sets also showed significant enrichment, mainly those describing genes regulated during loss of self-renewal capacity [241-243]. From general omics literature gene sets, significan enrichment was found for the "Lee_Neural_Crest_Stem_Cell_Up" set that contains genes up-regulated in neural crest stem cells (NCS, defined as p75+ HNK1+) [244], and the development-associated "cancer module 220" [245] 
Comparison of differential expression between normal (unexposed) ESTn time points showed that of the 100 genes in the ESTn enriched set, 22 genes peaked at $0 \mathrm{~h}, 43$ peaked at $24 \mathrm{~h}$, and 35 peaked at $48 \mathrm{~h}$. For the ESTn_restricted set, these numbers were 1,16 , and 12 , for $0 \mathrm{~h}, 24 \mathrm{~h}$ and $48 \mathrm{~h}$ respectively (Table 2 ). We retrieved STITCH database interactions between proteins, genes, and compounds using the ESTn_enriched as input and visualized this into molecular interaction network (Figure 4). This resulted in a network with 85 nodes (proteins, genes, compounds) and 182 edges (interactions). Of the network nodes, 66 are genes in the ESTn_enriched set, with the STITCH tool

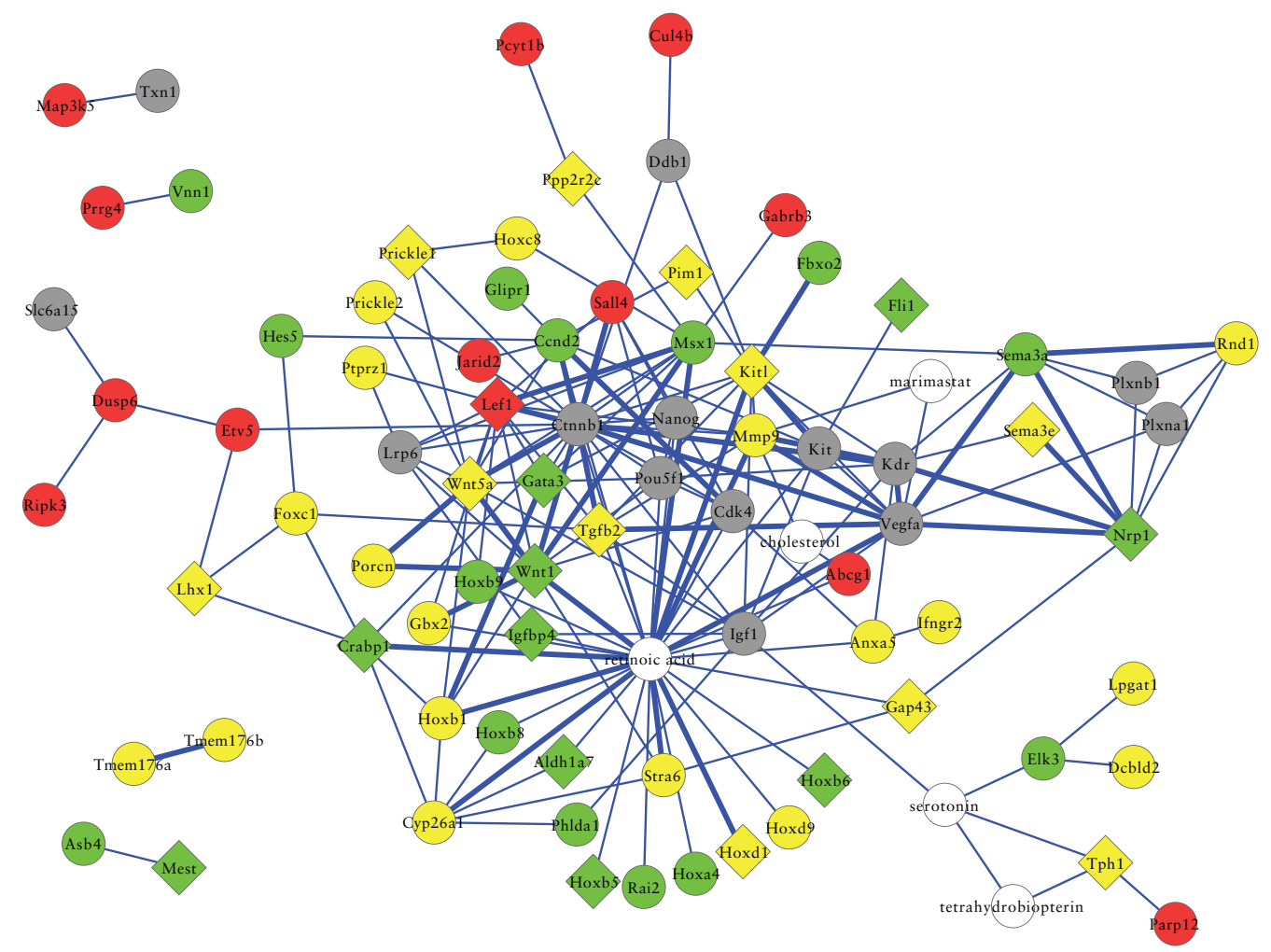

Figure 4 Molecular interaction network based on STITCH interactions for genes in the ESTn_enriched set. Narrow lines indicate moderate evidence (STITCH score $>0.4$ ) for interaction in either mouse OR human, wide lines strong evidence $(>0.7)$ in both mouse AND human. Nodes: red, $0 \mathrm{~h}$ peak expression; yellow, $24 \mathrm{~h}$ peak expression; green, $48 \mathrm{~h}$ peak expression; grey, protein added by STITCH; white, compound added by STITCH. Nodes shown as circles are only part of the ESTn_enriched set, nodes shown as diamonds are also part of the ESTn_restricted set. adding 5 compounds and 14 proteins that meet its default interaction thresholds. When expression and cross-validation data (Table 2) is overlaid on the network, it can be seen that the network is tightly connected around nodes with a role in development. Retinoic acid, introduced in the network by STITCH interaction analysis, has a central position and the highest connectivity (31 edges) in the network. Among its interactions are those with RA-metabolizing (Cyp26a1, Aldh1a7), and binding (Crabp1, Stra6) proteins, as well as several genes whose expression is affected by RA. Also in the network, othe central positions and/or high connectivity can be found for genes involved in (stem cell) development such as Wnt5a, Wnt1, Ctnnb1, Pou5f1, Nanog, Lef1, and Msx1; growth factors (Tgfb2, Kitl, Igf1, Vegfa) and growth factor receptors $($ Kdr, Kit)
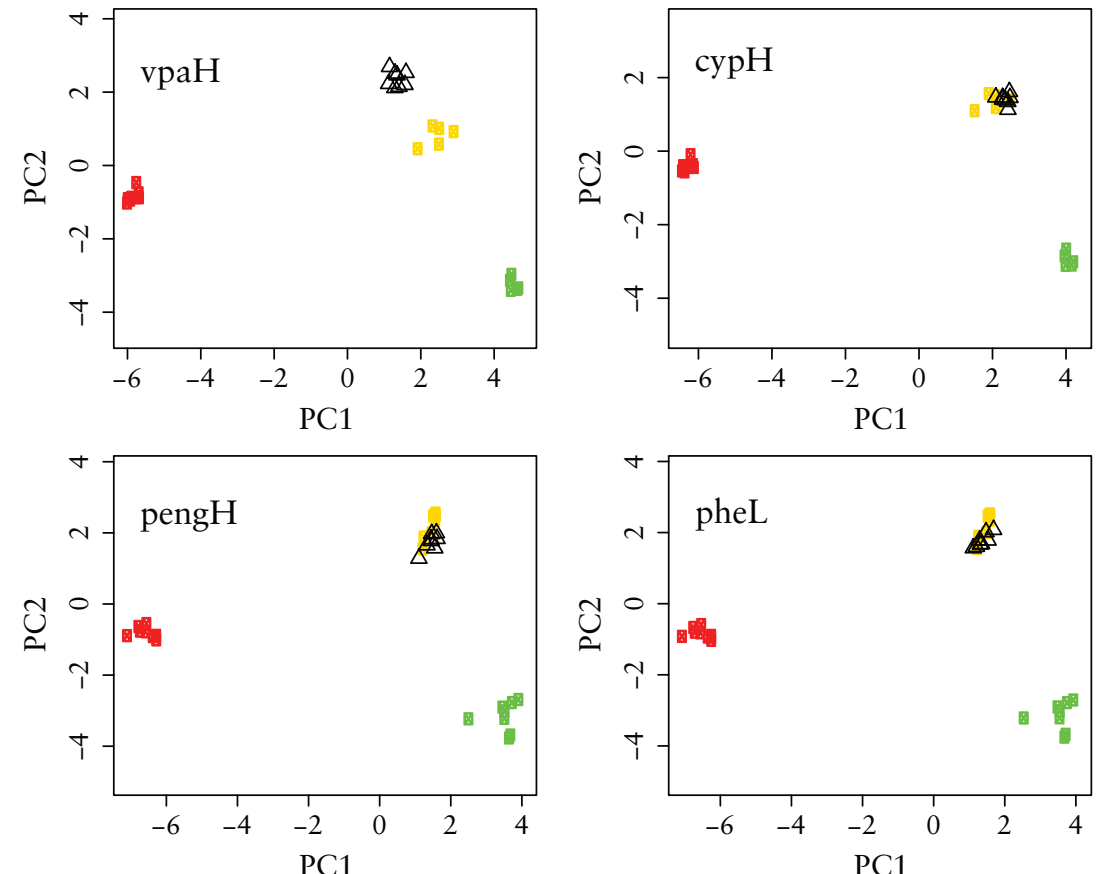

Figure 5 Examples of differentiation tracks for different misclassified compound exposures. Correctly classified valproic acid is shown in the upper left for comparison. Red circles, $0 \mathrm{~h}$ controls; yellow circles, $24 \mathrm{~h}$ controls; green circles, $48 \mathrm{~h}$ controls; black triangles, $24 \mathrm{~h}$ compound exposed samples. Labels are explained in Table 1. 


\section{Discussion}

$\mathrm{N}$ a number of previous studies, we introduced the use of transcriptomics in the ESTn [177, 203, 231]. In these papers, we also showed that the differentiation track algorithm previously developed for toxicity prediction in the classic (cardiac) EST, is effective in the ESTn as well. Given the possibilities for the ESTn as an alternative (neuro)developmental toxicity test system, further application of the differentiation track algorithm to the ESTn is of interest. Although current data are definitely not exhaustive, due to the wide range of molecular mechanisms and pathological outcomes known for neurodevelopmental toxicity, the current three studies allow including data of 10 compounds in various concentrations amounting to 19 exposure conditions in an integrated analysis, which should provide an indication of the ESTn predictive potentia at this moment. Moreover, such an integrated analysis would give an indication of which genes are most sensitive to different gene expression regulation by normal neural differentiation compared to neurodevelopmental toxican exposure, allowing focusing further research into in vitro screening assays.

The data used in this study contain both neurodevelopmental toxicants and non-toxicants ("positive" and "negative" compounds), but we also included exposures to non-toxic concentrations of neurodevelopmental toxicants as "negative" exposures. This is based on the notion that not the compound in itself but rather an "exposure", defined as a compound concentration or dose, is considered as a (developmental) toxicant [101]. By including both toxic (adverse) as well as merely adaptive exposure levels, this distinction can be linked to the magnitude of gene expression perturbation [226], i.e. the magnitude of track deviation, leading to better insights into the interpretation of in vitro assay results for risk assessment.

By sampling 200,000 gene sets and determining genes contributing to prediction accuracy, a set of 100 genes was obtained providing $74 \%$ prediction accuracy for the included data ( $\mathrm{p}$-value cutoff 0.05 ). Restricting the set by taking the intersection of cross-validation generated gene sets resulted in a smaller set of 29 genes providing $84 \%$ accuracy.

Among the incorrectly predicted exposures, misclassification of cyproconazole might be attributed to its properties of both being able to induce or inhibit neural differentiation in vitro, depending on the concentration [15, 203]. A look at the other misclassified compound exposures indicates these can be considered as 'borderline misclassified', such as the high concentration carbamazepine (ESTn_enriched only), high concentration penicillin G (both gene sets) and high concentration phenytoin (ESTn_enriched only). Upon closer examination, it was found that such borderline p-values correspond to a ow magnitude of track deviation (see for examples with the ESTn_restricted gene set Figure 5). In such cases, a borderline magnitude of the deviation (between $24 \mathrm{~h}$ exposed and control) might not lead to a correctly predicting p-value due to potential differences in intersample variation [84], indicating that such $\mathrm{p}$-values should be considered with more caution than more clear-cut positive or negative $\mathrm{p}$-values.

The biological relevance of the ESTn_enriched gene set is reflected in it functional enrichment for (neuro)developmental terms from GO as well as additional databases. For two thirds of these genes, a STITCH interaction was retrieved which, when visualized as a molecular interaction network (Figure 4), resulted in a compact network (diameter 7 links) that is strongly interconnected and built around a number of regulatory hubs in its center. The central position of RA and its high number of interactions (31, versus network average 4.3) corroborates the pivotal role of RA as a morphogen in the neura differentiation induction in the ESTn. Several additional network hubs are known as genes or proteins involved in development or growth, and are eithe part of the ESTn enriched set or have STITCH interactions with several of them (Figure 4, Table 2). Several relatively peripheral nodes added by STITCH are of interest in neural development as well, including the neurotransmitter serotonin, two plexin genes (Plxna1 and Plxnb1) involved in axon guidance [246-248], and Slc6a15 which belongs to a family of neurotransmitte (precursor) transporters [249, 250] and has been associated with susceptibility to major depression [251]. Furthermore, cholesterol is of importance during early embryogenesis and deficiencies in its metabolism have been associated with a range of (neuro)developmental effects [82, 252-256]. For most of the proteins added by STITCH, we found no significant gene expression changes during normal neural differentiation, and all of them showed only weak expression changes upon compound exposure (data not shown). This is in line with the finding that these genes are not found the be predictive in our ESTn model system. Instead, for these added nodes, the literature evidence for thei developmental function and interactions with ESTn_enriched genes should be interpreted as supportive evidence for the functional relevance of the network genes in neural differentiation. Altogether, the molecular concept network supports the observation that the majority of genes in the ESTn_enriched are directly or indirectly involved in (neural) development.

During unexposed differentiation in control cultures over time, the 100 genes regulated in the ESTn_enriched gene set peak in their expression at differen time points, which is consistent with one of the principles behind the differentiation track algorithm, namely allowing for continuous data visualization across a larger time window. Nevertheless, genes with an expression peak at 
$24 \mathrm{~h}$ are somewhat overrepresented (43/100 genes). This overrepresentation is also apparent in the more stringent ESTn_restricted set with 16 out of 29 genes having their highest expression at $24 \mathrm{~h}$. Such enrichment was not found in the Pennings_Diff_Correlation set obtained by a similar analysis on classic (cardiac) EST transcriptomics, where 24 and $48 \mathrm{~h}$ expression peaks (or those in between) were approximately equally present. Although comparative data between time points are limited, this emphasis on genes peaking at $24 \mathrm{~h}$ is in line with our finding that the transient gene expression changes at $24 \mathrm{~h}$ are clearly more sensitive to perturbation by methylmercury chloride compared to later time points in the ESTn [177]. For cardiac differentiation data after 24 and $96 \mathrm{~h}$ exposure to monobutyl phthalate and 6-aminonicotinamide, the $24 \mathrm{~h}$ time point was the more sensitive to transcriptomics changes, although the difference with the later time point was less pronounced [234].

The algorithms for gene set optimization and differentiation track definition are not based on a premise of similar gene expression responses across multiple compounds as there is increasing evidence that (neuro)developmental toxicity can occur through different effects on the transcriptome [82, 203, 231]. In fact, the key to the algorithm is that a set of genes whose expression is regulated during normal differentiation might (additionally) each be regulated in a different manner by different toxic compound exposure, provided the extent of overall gene expression perturbation is predictive for (neuro)developmental toxicity (see for examples Figure 2, Figure 3 or the figures in $[83,234]$ ). As the range of compounds tested vary considerably in mode of action regarding (neuro)developmental toxicity, gene expression changes for the various exposures to their matched control were also observed to be different (both qualitatively and quantitatively). Nevertheless, some similarities were found that are worth mentioning. Effects on RA-dependent genes by high concentrations of the three triazole compounds (flusilazole, cyproconazole and hexaconazole), such as their induction of Cyp26a1, Aldh1a7, and Rai2, are in agreement with the hypothesis that the triazole teratogenic mode of action lies in inhibiting common enzymes involved in RA metabolism, leading to disrupted RA homeostasis [225]. It should be noted that hexaconazole has developmentally toxic properties in causing skeletal malformations in rats, however no specific effects on neural development have been observed in vivo or in vitro [15, 257]. Similarities between acetaldehyde, carbamazepine and to a lesser extent - also valproic acid are also present. However, these cannot be linked to a structural similarity, although it might be noted that these compounds have all been associated with neural tube defects. Although compound response comparisons are not the primary purpose of this study, these similarities do indicate the possibility of deriving gene expression signatures for specific structural or phenotypic classes of (neuro)developmental toxicants and linking these to toxicity pathways [81, 203, 215, 231, 258-260]. Such signatures would be of additional interest for compound screening and risk assessment.

In summary, we have combined data from three transcriptomics studies in a single new analysis to identify an optimized gene set for neurodevelopmental toxicity prediction in the ESTn. This gene set allows for $84 \%$ prediction accuracy and consists mainly of genes involved in (neural) developmental processes. We anticipate this set will prove to be helpful to further improve studies into the ESTn as an in vitro assay for compound risk assessment.

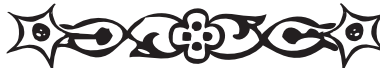




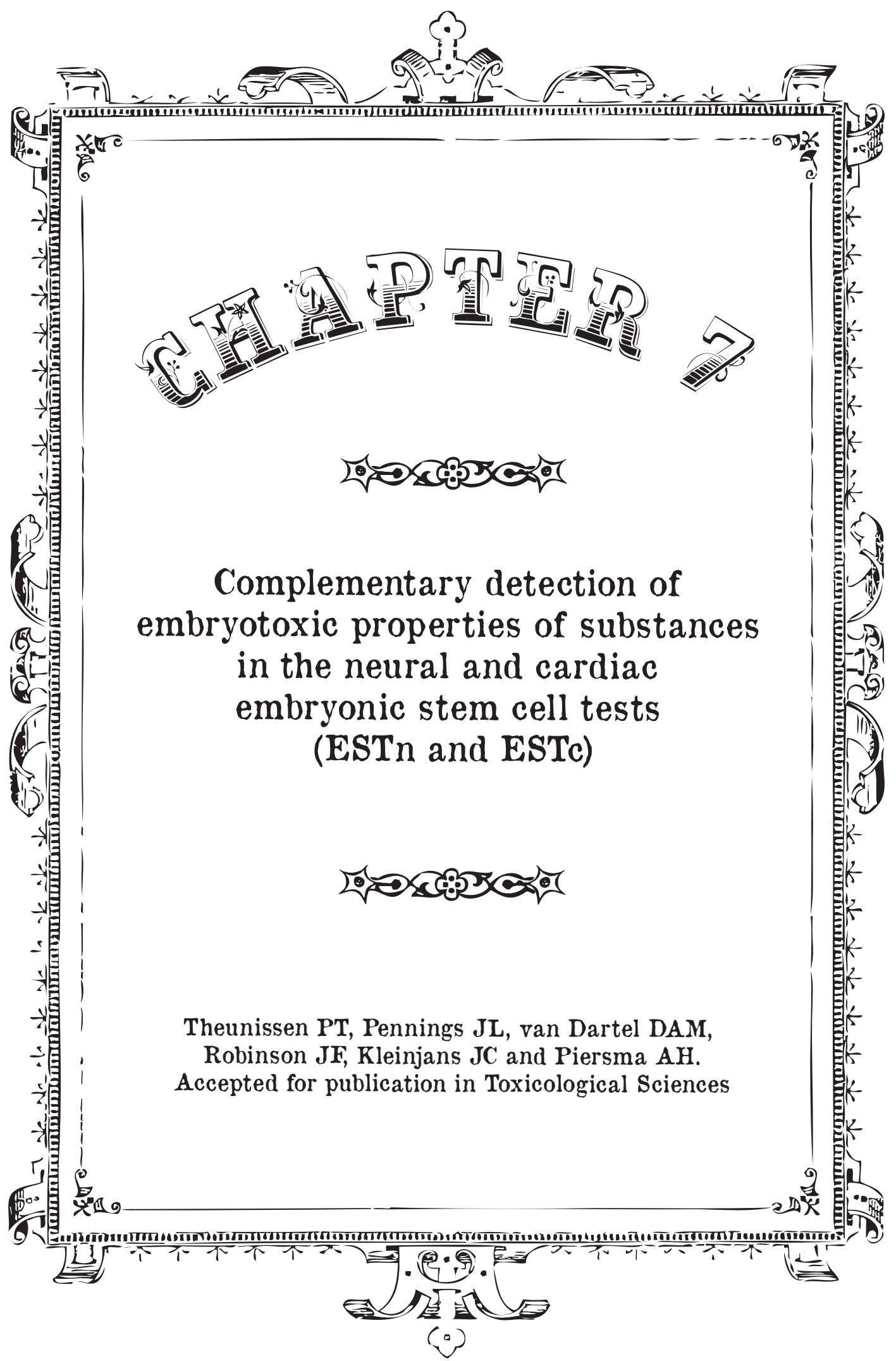




\section{Abstract}

$\mathrm{N}$ developmental toxicity testing, in vitro screening assays are highly needed to increase efficiency and to reduce animal use. A promising in vitro assay is the cardiac mouse embryonic stem cell test (ESTc), in which the effect of developmental toxicants on cardiomyocyte differentiation is assessed. Recently we developed a neural differentiation variant of the stem cell test (ESTn), and performed transcriptomic studies to characterize both ESTn and ESTc over time and to detect developmental toxicity in both models. Here, we compare the gene expression profiles of ESTn and ESTc over time in both methods as well as model-specific changes induced by seven compounds, comprising six in vivo developmental toxicants. Time-related gene expression profiles showed similarities between the two models. However, groups of genes could be identified responding differently in each model, mainly related to cardiac development in ESTc and neural development in ESTn, indicating characteristic differences between ESTn and ESTc. Furthermore, compound-induced gene expression changes were generally different in either model, such as for methylmercury and flusilazole, which were predicted better in ESTn and in ESTc, respectively. In addition, VPA induced gene expression changes were comparable in both ESTn and ESTc, but varied at the process level. This indicates that both ESTn and ESTc results complement each other. Therefore, a combined approach incorporating ESTc and ESTn may improve developmental toxicant detection over individual assays.

\section{Introduction}

URRENT chemical hazard assessment for developmental toxicity is built upon globally harmonized OECD animal test guidelines, providing a structure for chemical risk assessment. Under the current European chemical safety regulation guidelines (REACH, Regulation, Evaluation and Authorization of Chemicals), approximately $60 \%$ of the animals used for toxicity testing are estimated to be required for reproductive and developmental toxicity studies [261]. In order to reduce the number of experimental animals needed for developmental toxicity testing, alternative test systems have been developed, including the embryonic stem cell test (EST) [17]. In this test system compound perturbation of mouse embryonic stem cell (ESC) differentiation into cardiomyocytes is determined by manually counting beating cell foci. In the first in ter-laboratory EST validation tests, performed under supervision of the European Centre for Validation of Alternative Methods (ECVAM), the model predicted compound developmental toxicity with an overall accuracy of $78 \%$. However, one of the compounds misclassified in four out of eight experiment was methylmercury $(\mathrm{MeHg})$, a model neurodevelopmental toxicant [17]. In workshops following the validation studies, attempts were made to discern the applicability domain of the EST and to determine the improvements deemed necessary to increase prediction and throughput of the EST [36, 37]. Proposed improvements included integration of objective molecular parameters into the EST as predictors for developmental toxicity and development of ESC developmental toxicity assays using alternative differentiation methods into the ectodermal and endodermal lineage, including neural differentiation [36, 37].

Previously, we developed a neural variant of the cardiac differentiation EST (ESTc), the neural embryonic stem cell test (ESTn) [142]. The ESTn was designed to complement the ESTc and therefore, culture methods were kept comparable to the ESTc, including embryoid body (EB) formation using hanging drop culture, EB suspension phase and plating of the EB [142]. In order to promote neural differentiation and repress mesodermal differentiation, a physiological concentration of $0.5 \mu \mathrm{M}$ all-trans retinoic acid (RA) was added to the culture during the suspension phase [262]. In addition, medium serum deprivation was used to further select for neural differentiated cell types.

In order to characterize the ESTn and ESTc, transcriptomics studies were performed, analyzing global gene expression patterns in both models over time $[40,177,187]$. These studies formed the basis for the differentiation track, using principal component analysis (PCA) to describe differentiation over time in both models [187]. Furthermore, transcriptomics was used to study effects 
of known developmentally toxic compounds on cardiac and neural differentiation in ESTc and ESTn, respectively [83, 155, 177, 203, 263]. Compound effects on gene expression were determined using the differentiation track algorithm by calculating the deviation of $24 \mathrm{~h}$ exposed samples to the differentiation track consisting of $0 \mathrm{~h}$ (day 3), 24h (day 4) and 48h (day 5) control samples [187]. Using this approach, developmental toxicity of 17 and 10 compounds has been evaluated in ESTc and ESTn, respectively, of which 7 compounds have been tested in both models $[83,155,177,203,263]$. Due to the similarities in design between ESTc and ESTn and comparable exposure scenarios using the differentiation track algorithm, comparisons between the two models are straightforward. In this study, we aim to characterize differences and similarities in global gene expression changes between the ESTc and ESTn systems over time in control and exposed situations.

\section{ఝి}

\section{Materials and Methods}

\section{Microarray samples and analysis}

Methods and data regarding ESTc and ESTn culture conditions, compound exposures, assessment of differentiation inhibition, and microarray transcriptional profiling have been described in full detail in previous publications by our group [83, 155, 177, 203, 263]. Raw and normalized Affymetrix microarray data used in this study are available at ArrayExpress (www.ebi.ac.uk/), under accession numbers E-MTAB-300, E-TABM-1027, E-TABM-1108, E-TABM1205, and E-TABM-1216. As the five data sets used are not fully identical in experimental design regarding time- and concentration points, some restriction were applied regarding comparable time-courses and exposure scenarios, to ensure comparability. Unexposed controls at 0,24 , and $48 \mathrm{~h}$, calculated from 3 days after the start of the protocols, were included for each of the five studies; compound exposed samples at $24 \mathrm{~h}$ were included for compounds studied in both ESTc and ESTn at the concentration resulting in a $50 \%$ inhibition of differentiation (ID50) for developmental toxicants or a maximally feasible concentration without affecting cellular viability for non-developmenta toxicants. This led to 246 out of 555 samples being selected for further analyses. An overview of selected compounds and concentrations is provided in Table 1. All statistical calculations used in this study were performed in $\mathrm{R}$ (http://www.R-project.org) unless indicated otherwise.

\section{Data Analysis and Statistics}

Affymetrix CEL files were normalized using the Robust Multichip Average (RMA algorithm [158] using RMAexpress [159]. For probe to gene mapping, a custom Chip Description File (CDF) was used according to the assembly by de Leeuw et l. [160] (http://mad-db.science.uva.nl/ wdeleeuw/HybridAnnot/version6.html). Of the hybrid probe-set definitions included in the custom annotation, 16,331 probe sets defined by the Brainarray custom CDF version 11 (http://brainarray.

Table 1 Included data for compounds tested and numbers of genes regulated

\begin{tabular}{|c|c|c|c|c|c|c|c|c|c|c|c|}
\hline \multirow[b]{2}{*}{ Compound } & \multirow[b]{2}{*}{ Abbreviation } & \multirow[b]{2}{*}{$\begin{array}{l}\text { In vivo } \\
\text { classification }\end{array}$} & \multicolumn{3}{|l|}{ ESTn } & \multicolumn{6}{|l|}{ ESTc } \\
\hline & & & Concentration & $\begin{array}{l}\text { Concentration } \\
\text { Selection }\end{array}$ & $\begin{array}{l}\text { \# genes } \\
\text { regulated } \\
\text { FDR }<0.05 \text {; } \\
\mathrm{p}<0.001\end{array}$ & Concentration & $\begin{array}{l}\text { Concentration } \\
\text { Selection }\end{array}$ & $\begin{array}{l}\text { \# genes } \\
\text { regulated } \\
\text { FDR }<0.05 ; \\
\text { p }<0.001\end{array}$ & Overlap & $\%$ in ESTn & $\%$ in ESTc \\
\hline Carbamazepine & $\mathrm{CBZ}$ & Teratogen / NDT & $100 \mathrm{uM}$ & ID50 & 531 & $160 \mathrm{uM}$ & ID50 & 1117 & 148 & $28 \%$ & $13 \%$ \\
\hline Flusilazole & FLU & Teratogen & $10 \mathrm{uM}$ & IC20 & 994 & $5.4 \mathrm{uM}$ & ID50 & 588 & 105 & $11 \%$ & $18 \%$ \\
\hline Hexaconazole & HEX & Teratogen & $25 \mathrm{uM}$ & IC20 & 1028 & $21 \mathrm{uM}$ & ID50 & 687 & 90 & $9 \%$ & $13 \%$ \\
\hline Methylmercury & $\mathrm{MeHg}$ & NDT & $0.025 \mathrm{uM}$ & ID50 & 618 & $0.48 \mathrm{uM}$ & ID50 & 605 & 33 & $5 \%$ & $5 \%$ \\
\hline Monoethylhexylphthalate & MEHP & Teratogen & $100 \mathrm{uM}$ & ID50 & 16 & $680 \mathrm{uM}$ & ID50 & 844 & 4 & $25 \%$ & $0 \%$ \\
\hline Penicillin G & PenG & Non-Teratogen & $1000 \mathrm{uM}$ & Max conc & 1 & $1100 \mathrm{uM}$ & Max conc & 536 & 0 & $0 \%$ & $0 \%$ \\
\hline Valproic acid & VPA & Teratogen / NDT & $1000 \mathrm{uM}$ & ID50 & 1925 & $800 \mathrm{uM}$ & ID50 & 4133 & 919 & $48 \%$ & $22 \%$ \\
\hline
\end{tabular}

NDT: Neurodevelopmental Toxicant 
mbni.med.umich.edu/Brainarray/Database/CustomCDF) [161] and 4648 additional probe sets defined by Affymetrix chip annotation 26 were used in further analyses, giving a total of 20,979 probe sets. Probe sets for Affymetrix internal controls or probe sets that did not correspond to an Entrez Gene ID were not used in further analyses. For each gene, maximal fold change (FC) in gene expression between the experimental groups were determined by comparing the average normalized signal values per group and were calculated as the maximum/ minimum ratio. For gene expression over time, samples were compared to the mean expression of all time samples. For compound toxicity effects, the treated samples were compared to the time matched day 4 controls. Genes differentially expressed between any of the experimental groups were identified by a one-way ANOVA. A significance threshold with a false discovery rate (FDR) of FDR $<0.01$ and $\mathrm{FC}>1.5$ was used to select genes significantly differentially expressed over time. Genes expressed over time in all three ESTn and/or both ESTc experiments were used for further analysis (Figure 1A). Genes regulated over time were ranked using hierarchal clustering and definition of gene expression clusters with a simila expression profile over time was performed using a hierarchal clustering branching cutoff at 10 branches. A significance threshold of FDR $<0.05$ and $p<0.001$ was used to select genes significantly differentially expressed due to compound exposure compared to the day-matched control. For further analysis of gene expression changes by specific compounds (FLU, MeHg and VPA) all genes significantly regulated by the compound were included (FDR $<0.05$ and $\mathrm{p}<$ 0.001), without the additional time criteria. Hierarchical clustering of changes to control for all significantly differentially expressed genes was performed in GeneMaths XT (Applied Maths, Sint-Martens-Latem, Belgium), using Euclidean distance and Ward linkage. Venn diagrams describing relations of significant gene expression were created using Venny [214].

Functional annotation and enrichment for Gene Ontology biological processes (GOBP) were studied using DAVID (http://david.abcc.ncifcrf.gov/) [92] Significantly enriched GOBP categories were based on a set criteria of $\mathrm{p}$-value ( $\mathrm{p}<$ 0.01 ), and genes changed within each specific gene ontology category $(>5)$. In order to visualize GOBP enrichment, GOBP were separated into the following groups: "general development" (including broad developmental terms not describing specific organs or cell types), "neural development", "cardiac/ vasculature and mesenchymal development", "other development" (including development and differentiation related to organs and cell types other than neural or cardiac), "transcription", "metabolic, biosynthetic and catabolic processes" and "other" GOBP (including all GOBP not involved in the earlier defined criteria). Involvement of individual genes in these pathways was determined at GeneCards (www.genecards.org) [90].
Gene expression differences between ESTn and the ESTc were further characterized using principal component analysis (PCA) to visualize them as a differentiation track, as was described earlier [187]. In this analysis, a principal component is defined as a mathematically derived combination of genes and their expression characteristics which can be used to depict part of the proces observed. A number of principal components that are mutually independen can be derived which in combination describe the process under study. For the differentiation track comparison was based on those identified to be significantly differentially expressed in all three ESTn experiments or both ESTc experiments during differentiation over time sampled at days 3,4 and 5 (FDR $<0.01, \mathrm{FC}>1.5$ ). PCA analysis was performed with these genes, resulting in two curves describing the differentiation track for either ESTn or ESTc.

A network with gene, protein and compound interactions was built in Cytoscape using data obtained from the STITCH database [239] (http://stitch. embl.de/). We used the gene symbols enriched in clusters B and $\mathrm{G}$ as input, to let the STITCH search tool determine mutual interactions among the input genes as well as proteins and chemicals that the STITCH search tool finds associated with them. This query was performed using the mouse as organism, a confidence score of 0.300 and otherwise default settings. STITCH output files were reformatted for input and visualization in Cytoscape [240].

\section{\&8}

\section{Results}

Selection of genes regulated over time in ESTn and ESTc

Genes significantly regulated over time (day 3, day 4 and day 5 of the protocols, FDR $<0.01 ; \mathrm{FC}>1.5$ ) in the ESTn and ESTc were determined for three and two separate experiments, respectively (Figure 1A). In the three ESTn experiments, 3297, 3903 and 3977 genes were regulated over time, of which 2453 were commonly regulated over three experiments. In the two ESTc experiments, 3509 and 3754 genes were regulated time-dependently, of which 2675 were commonly regulated in both experiments. The 2675 and 2453 genes commonly regulated in the ESTc and ESTn, respectively, were used for furthe analysis, leading up to a combined total of 3648 genes to be further analyzed. In total, 1480 genes were commonly significantly regulated over time in both models, comprising $60 \%$ of ESTn and $55 \%$ of ESTc temporally regulated genes, respectively. 


\section{Common and unique gene expression changes between ESTn and ESTc}

Common and model-specific global gene expression changes over time in ESTn and ESTc were investigated. Changes of time-dependent gene expression were visualized using PCA (Figure 1B). The first and second principal components (PC1 and PC2) together were responsible for $71 \%$ of gene expression variation. Day 3 samples of all experiments, both ESTn and ESTc, clustered together. Day 4 and day 5 samples followed distinct separate paths corresponding to differences in the ESTn versus ESTc differentiation culture conditions. For each time-point in both models, samples clustered together, demonstrating limited variation within and among experiments in both models. Hierarchal clustering of the 3648 genes regulated by either ESTn and/or ESTc is shown in figure 1C. Ten gene clusters were identified, each describing distinct patterns of gene expression changes over time in both ESTn and ESTc. For instance, clusters B, D and E were mainly regulated over time in ESTc $(17 \%$ of al time-regulated genes), while clusters $\mathrm{F}$ and $\mathrm{G}$ were mainly regulated over time in the ESTn (12\% of all time-regulated genes). Furthermore, clusters A, C, H, $\mathrm{I}$ and $\mathrm{J}$ showed similar gene expression responses over time for both models, indicating these genes are involved in processes regulated in both models, independent of different culture conditions. Number of genes and number of GOBP regulated varied between clusters. GOBP enrichment analysis of genes regulated within the clusters showed that in all clusters a substantial part of enriched GOBP was involved in General Development, Transcription and Metabolic Processes (Figure 1D). In addition, GOBP in cluster $\mathrm{H}$ were enriched for cell death and apoptosis. Specifically, the number of GOBP related to neura development were enriched in clusters A-G, and most prominently in cluster $\mathrm{F}$, whereas GOBP related to cardiac, vasculature and/or mesenchyme development were enriched in clusters A-F, most prominently in clusters B and D. The increased amount of enriched GOBP related to neural (cluster G) and cardiac (clusters B and D) related development was in correspondence with the gene expression profiles in these clusters as observed in figure 1C.

Further analysis of the two most responsive clusters B and G was performed at the gene expression level (Figures $1 \mathrm{E}$ and $1 \mathrm{~F}$ ). These clusters contained the fewest genes regulated as compared to other clusters, but were enriched for a relatively large number of GOBP (86 and 47). Genes in cluster B were involved in cardiac, vasculature or mesenchymal development (23 genes) (e.g. Myl7, Mesp2 and Nrp1), development related signaling pathways (15 genes), including the TGF -signaling pathway (e.g. Bmp2, Bmp7, Fgf3, Fgfb2), Wnt-signaling pathway (Wnt2, Wnt5a, Frzb) and homeobox gene expression (Lhx1, Msx2,
Cdx2) and other organ/cellular development (9 genes) (GATA6, Foxc1, Foxc2, Igf2). In addition, 8 genes were involved in neural development, of which 4 genes were also involved in cardiac development (Cfc1, Snai2, Gpr124 and Hey2). Furthermore, in cluster B, all 77 genes were significantly regulated ove time in ESTc and 46 of these genes were also significantly regulated in ESTn. Genes regulated over time in ESTn showed a much lower FC compared to FC in ESTc. Genes in cluster G were mainly homeobox genes (24 genes) (e.g. Hoxb, Hoxc and Hoxd family genes, Pax 3, Pax6, Mnx1), involved in neura development (10 genes) (e.g. Col2a1, Foxd1) and retinoic acid signaling (9 genes) (e.g. Crabp1, Cyp26b1, Nr2f1, Rarb). In cluster G, all 70 genes were significantly regulated over time in ESTn and 49 of these genes were also significantly regulated in ESTc. Genes regulated over time in ESTc showed a much lower FC compared to that in the ESTn. Furthermore, genes significantly regulated by both ESTn and ESTc were upregulated from day 4 onwards in ESTn, but only upregulated on day 5 in ESTc.

In order to investigate functional connections between the genes within cluster B and G, interaction maps were constructed using STITCH and Cytoscape. Approximately $70 \%$ of the genes in both clusters were involved in the interaction mapping. For cluster B, genes involved with Wnt-, BMP- and TGF signalling pathways clustered together, with cardiac, vasculature and mesoderm differentiation related genes mainly connected to these genes. In cluster $G$, genes were mainly connected to RA, which was added by STITCH as a highly associated compound, and consisted mainly of RA signaling and homeobox genes, whereas genes related to neural development were connected at the rim of the network (Figure $1 \mathrm{G}$ and figure $1 \mathrm{H}$ ). 
A

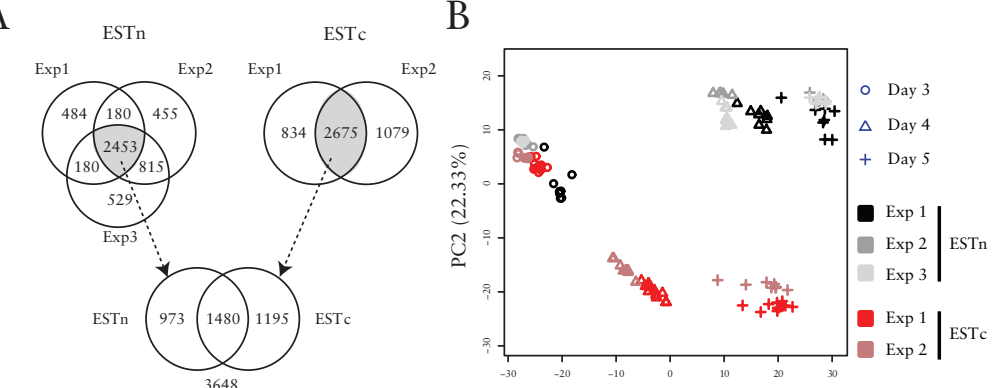

C

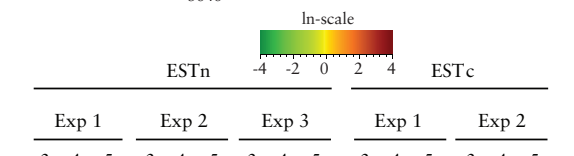

PC1 (49.05\%)
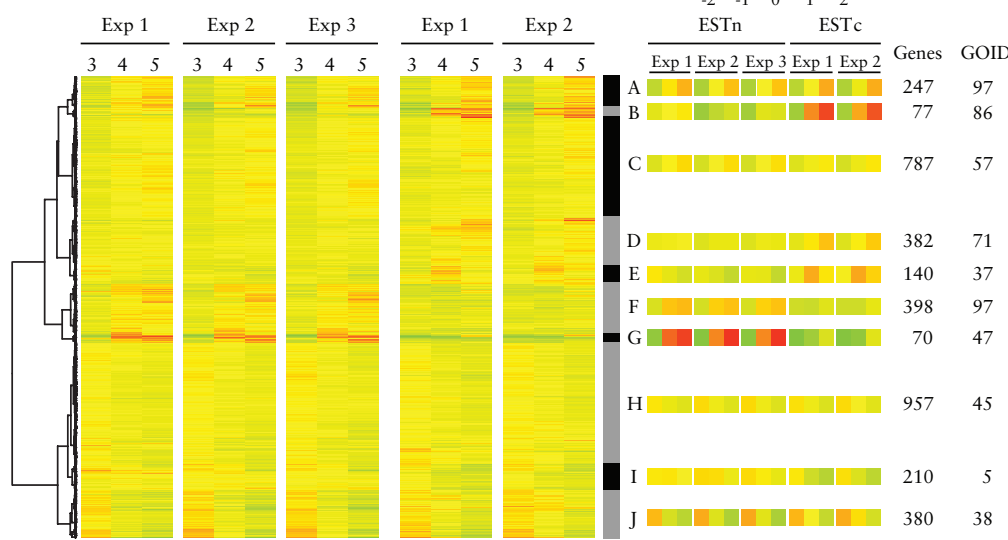

D

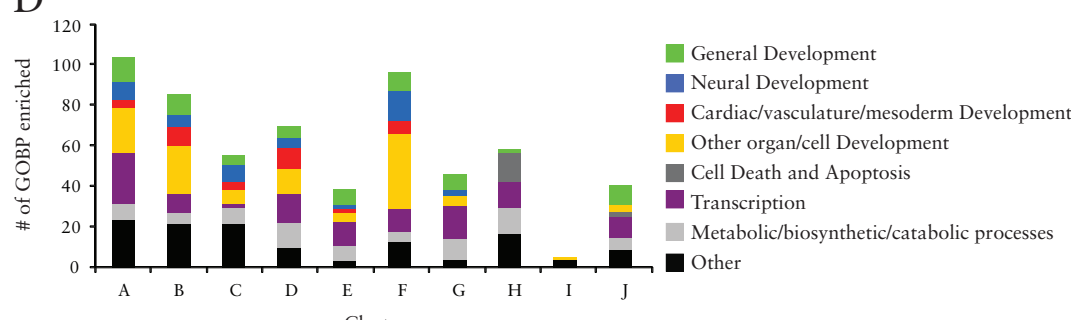

Cluster

Figure 1 Time-related gene expression changes in ESTn and ESTc. A) number of genes regulated over time (day 3, 4 and 5) in three ESTn experiments and two ESTc experiments (FDR < 0.01; FC > 1.5). Genes (3648) expressed in all three ESTn experiments and in both ESTc experiments were used for further analysis. B) PCA of samples taken at days 3, 4 and 5 in ESTn and ESTc, comprising 3648 genes regulated by ESTn and/or ESTc, describing the differentiation tracks for ESTn and ESTc experiments. C) Hierarchal clustering of the 3648 genes regulated over time in either model. The average gene expression of the top ten clusters (A-J) is provided for each experiment. Number of
E

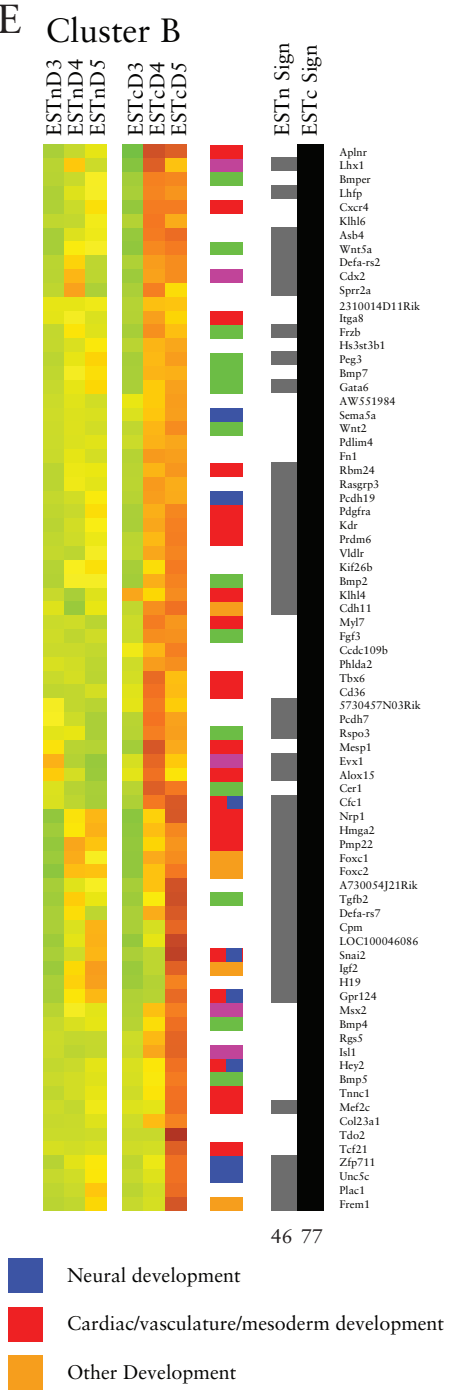

Cluster G

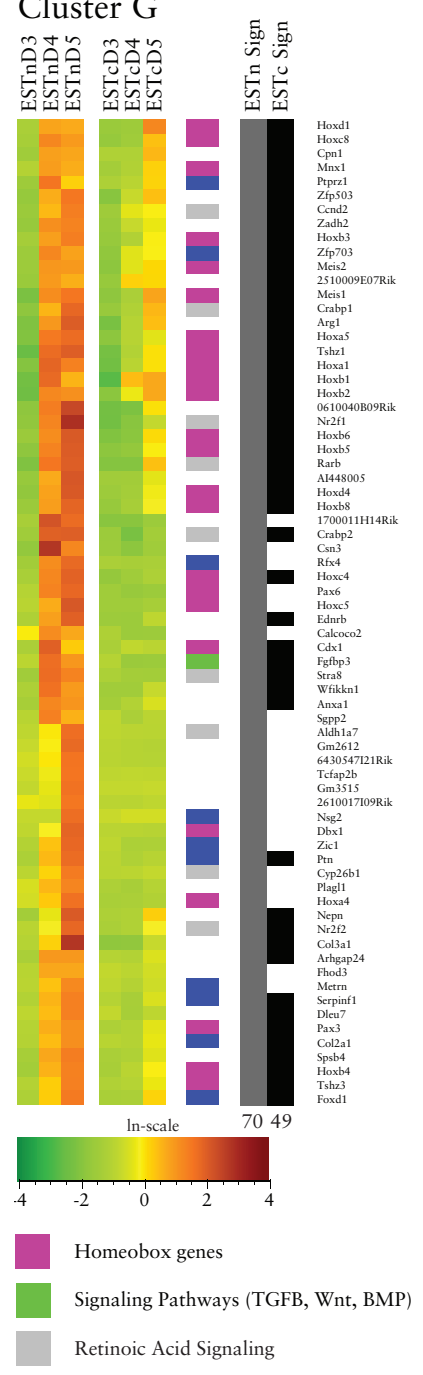

genes per cluster and GOBP regulated by these clusters are provided. D) Number of GOBP regulated in each cluster related to general development, neural development, cardiac/vasculature/mesodermal development, other organ/cell development, cell death and apoptosis, transcription, metabolic/biosynthetic/catabolic processes or other processes. E) Genes regulated in clusters B and G. Gene order is based on hierarchal clustering in figure 1C. Gene expression, function and significance (ESTn = grey, ESTc = black) are shown. 
G

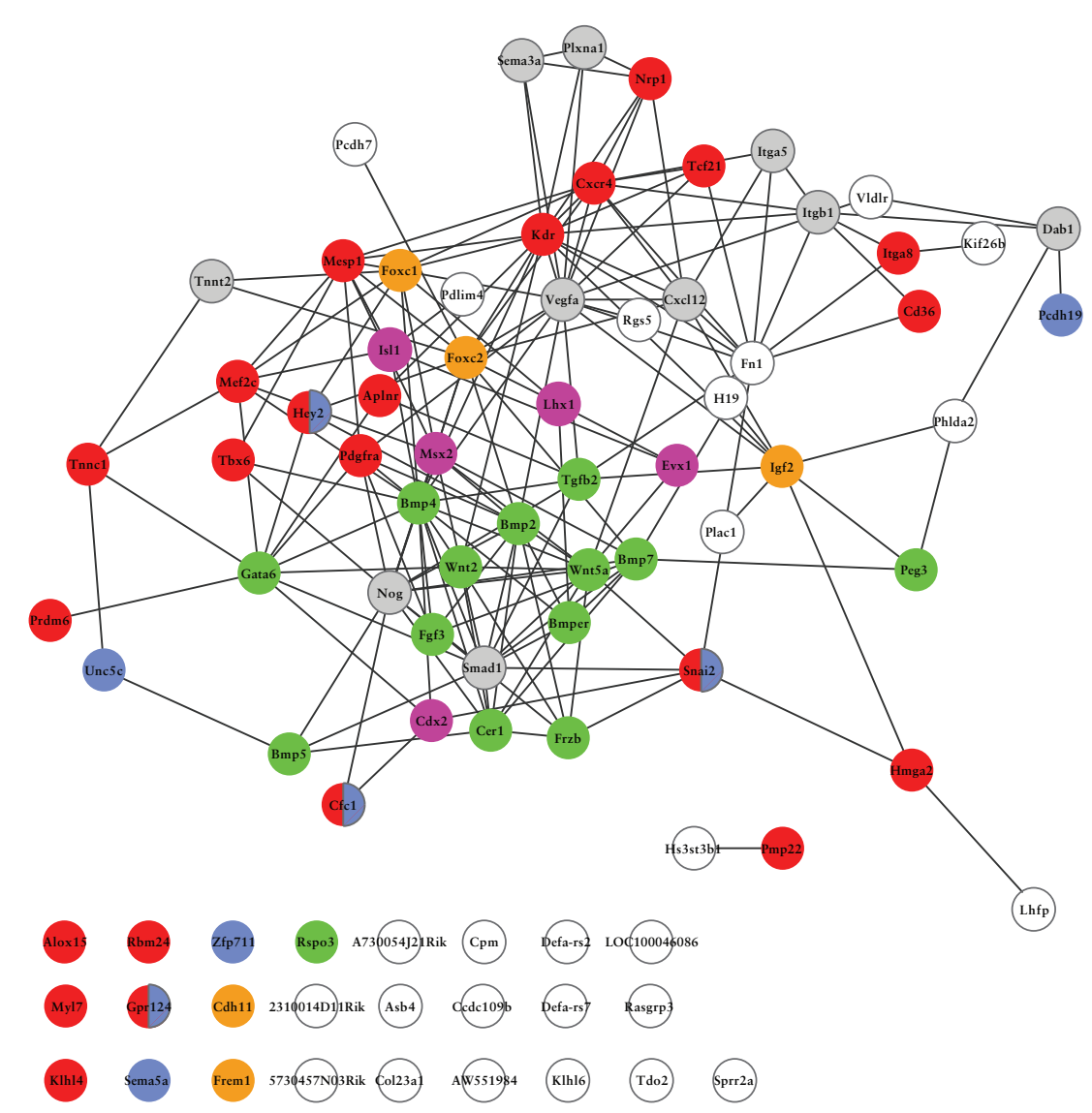

Figure 1 Molecular interaction network based on STITCH interactions for genes in sets cluster B (Figure 1G) and cluster G (Figure 1H). Lines indicate at least moderate evidence (STITCH score $>0.3$ ) for interaction in mouse. Compounds (square) and genes (circles) added by STITCH due to a very high connection are marked grey with black border.
$\mathrm{H}$

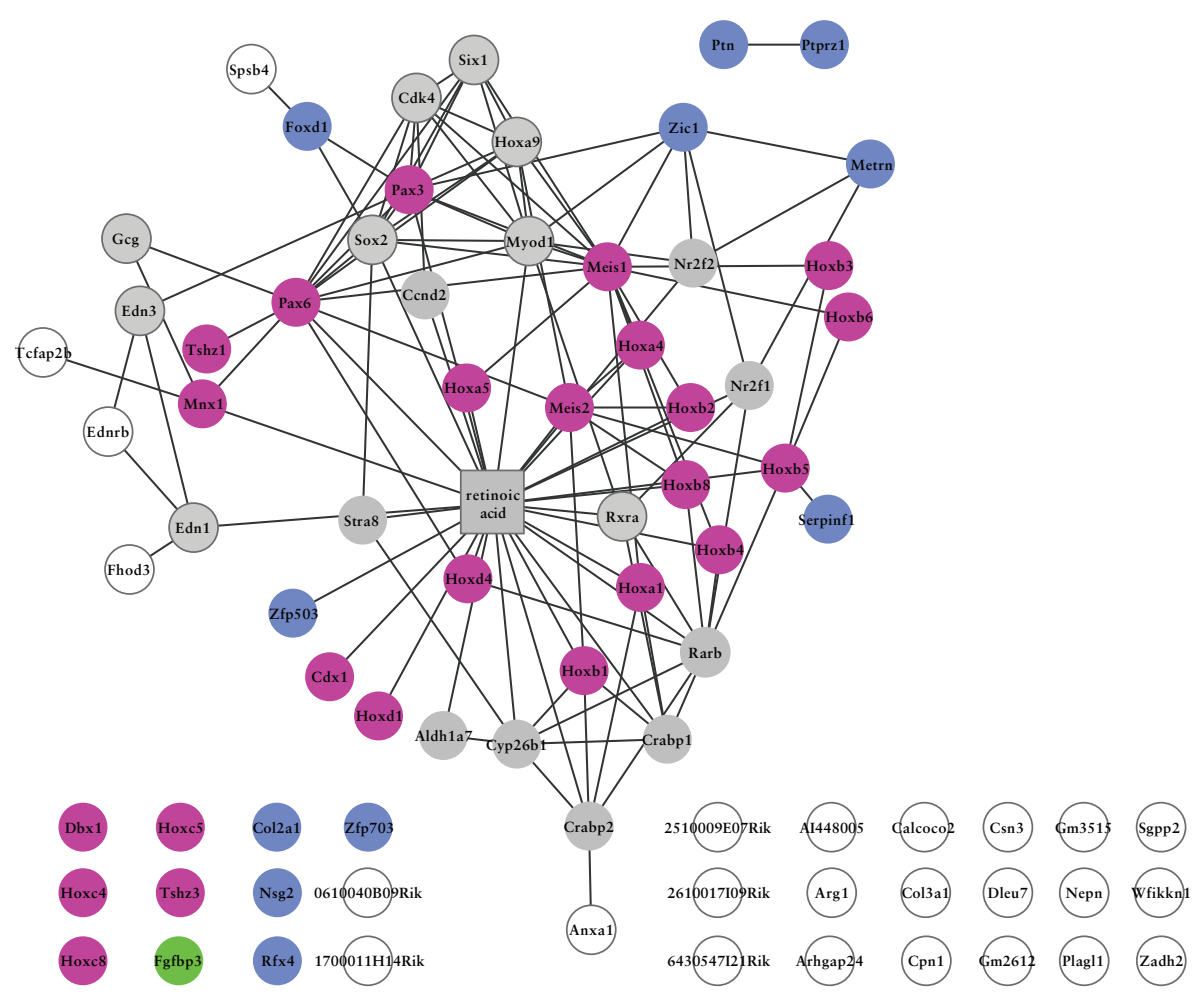

Neural development

Cardiac/vasculature/mesoderm development Other organ/cell Development

Homeobox genes

Signaling Pathways (TGFB, Wnt, BMP)

Retinoic Acid Signaling

Other

$\bigcirc / \square$ Added by STITCH 


\section{GOBP enrichment over time in ESTn and ESTc}

Differences in significance of GOBP enrichment between the two models was examined in a clustering plot $(-\log (\mathrm{FDR}))$ (Figure $2 \mathrm{~A})$. In general, GOBP involved in cardiac development were more significantly enriched in the ESTc, including the GOBPs Heart Development (ESTn FDR 7.8*10-7; ESTc FDR

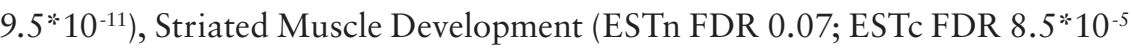
and Mesoderm Development (ESTn FDR 2.4 ; ESTc FDR:8.0*10-6). GOBP involved in neural development were more significantly enriched in the ESTn, including GOBP Regulation of Neuron Differentiation (ESTn FDR 2.3*10-8; ESTc FDR 0.006). GOBP involved in general development were more evenly regulated between the ESTn and ESTc. Overall, GOBP enriched related to general development were significantly enriched at a comparable level, such as Embryonic Morphogenesis (ESTn FDR 5.0*10-22 ; ESTc FDR 1.0*10-23). However, the GOBP Gastrulation and Formation of Primary Germ Layer were significantly regulated in ESTc (FDR $1.2^{*} 10^{-6}$ and $4.8^{*} 10^{-6}$, respectively) but

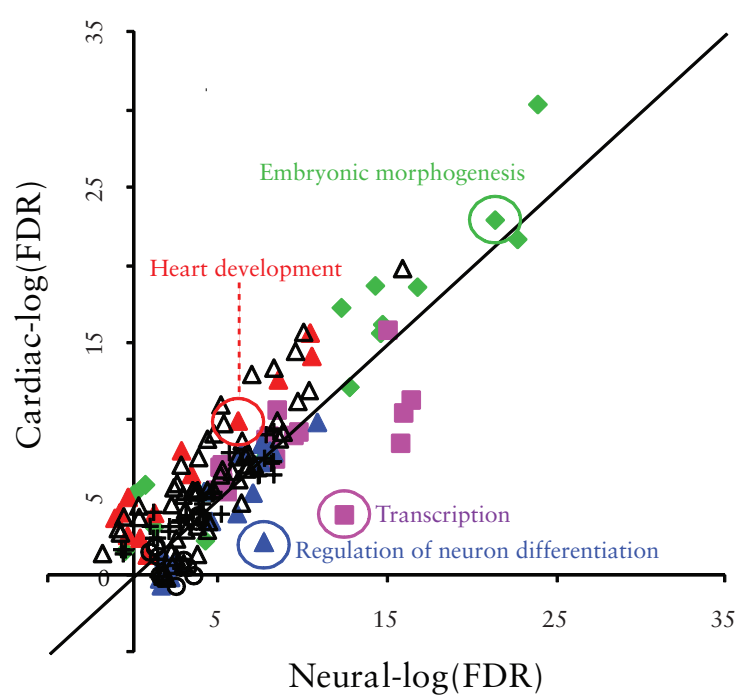

- General Development(14) $\quad \Delta$ Other Development $(60)$

- Transcription (16) O Cell death \& apoptosis (13)

$\Delta$ Neural development (18) + Other (34)

Cardiac/vasculature/mesoderm Development (14)

Figure 2 Clustering of GO biological process enrichment significance $(F D R<0.05$ in one of the models) over time (days 3, 4 and 5) between ESTn and ESTc not in ESTn (FDR 0.07 and 0.23, respectively). Further analysis showed that all significantly regulated genes within the GOBP Mesoderm Formation (20 genes) were part of the Gastrulation GOBP (data not shown). Four GOBP related to transcription, including the GOBP Transcription itself, were more significantly enriched in the ESTn (FDR 4.1*10-13) compared to the ESTc (FDR $\left.1.0 * 10^{-4}\right)$.

Compound- and time induced gene expression changes in ESTn and ESTc

In total, seven compounds previously studied for developmental toxicity in the ESTn and ESTc using a comparable transcriptomic approach were included in the analysis (Table 1) $[83,155,177,203,263]$. Ideally, concentrations at which compound effects were compared between assays were equipotent (ID50) in terms of morphological end point of ESTn and ESTc, respectively. For all compounds equipotent concentrations were within the same order of magnitude in both assays, except for $\mathrm{MeHg}$, which appeared over an order of magnitude more potent in ESTn as compared to ESTc. For flusilazole and hexaconazole, in ESTn cytotoxicity occurred at concentrations lower than those at which effects on differentiation were observed. Therefore, for these two compounds, the IC20 in ESTn was used for comparison with ESTc to compare simila effective concentrations in both assays and to avoid the analysis being dominated by cell viability-related genes rather than differentiation-related genes. The number of genes regulated by a compound in $(48 \%$ of genes regulated in ESTn and 22\% of those regulated in ESTc). $\mathrm{MeHg}$ induced gene expression showed a low overlap, with only 33 genes regulated by both models ( $5 \%$ of genes regulated in both ESTc and ESTn), despite a similar number of genes regulated in both models. In total, 6748 and 9380 genes (FDR $<0.05$ were significantly regulated by any compound in ESTn or ESTc, respectively. Furthermore, of these compound-regulated genes, 1718 and 1808 genes were additionally regulated over time in ESTn or ESTc, respectively (Figure 3A). Genes in these sets were therefore involved in differentiation in either model and regulated by at least one compound. In total, 2720 genes were regulated time-dependently and by at least one compound in both ESTn and ESTc. Gene expression profiles of these 2720 genes are shown as a hierarchal clustering plot in figure $3 \mathrm{~B}$.

To further illustrate differences in compound induced gene expression changes between the two models, compound specific effects of MeHg, FLU and VPA on gene expression in ESTn and ESTc are shown (Figure 4). MeHg and FLU both induced dissimilar gene expression profiles in ESTn and ESTc, with only limited overlap in significantly altered genes between models (Table 1). When 


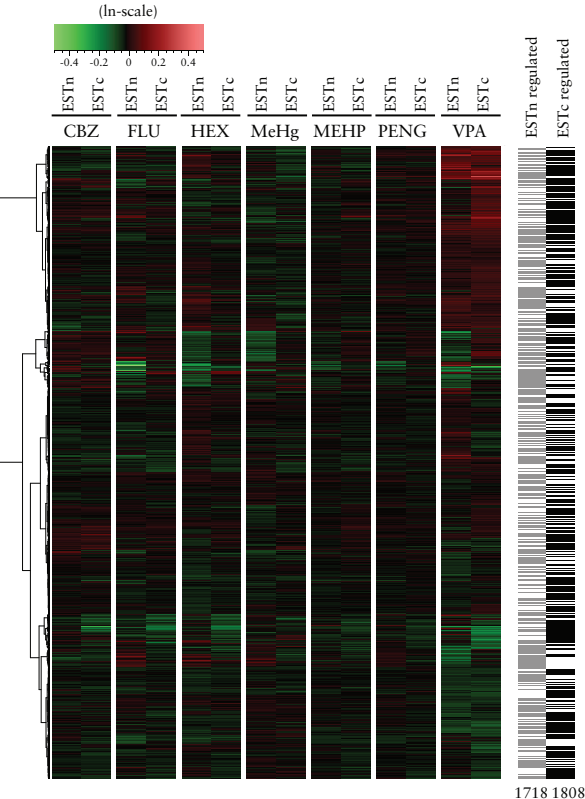

A
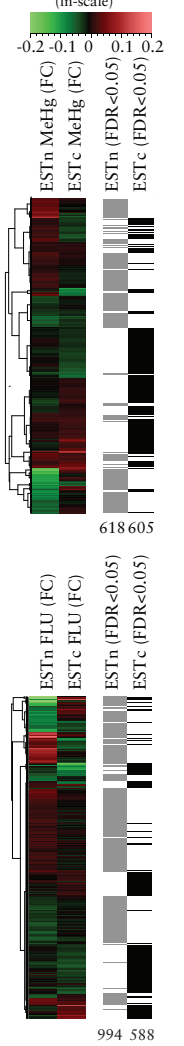
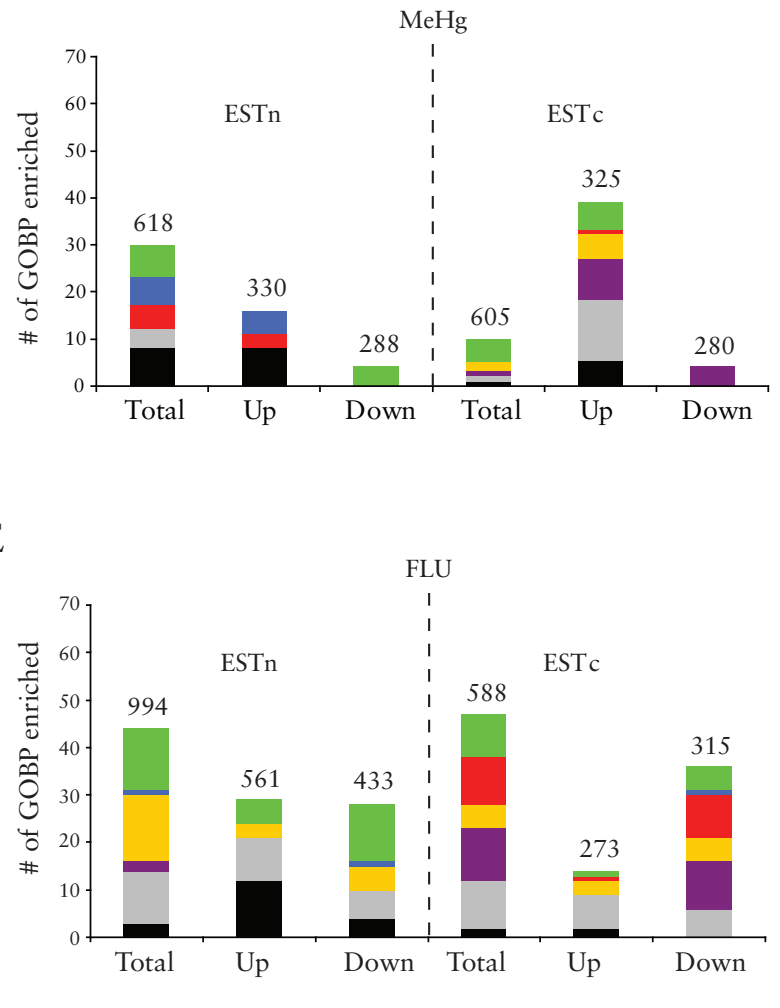

Figure 3 Time and compound related gene expression in ESTn and ESTc. A) Venn diagrams of genes regulated over time (day 3,4 and $5 ; \mathrm{FDR}<0.01 ; \mathrm{FC}>1.5$ ) and by any compound (compared to day-matched control; FDR $<0.05$ ) for both ESTn and ESTc experiments. 1718 and 1808 genes were regulated over time and by any compound in ESTn and ESTc, respectively. 806 of these genes were commonly regulated over time and by compounds in both models B) Hierarchal clustering of the 2720 genes over time and by compounds in either ESTn and/or ESTc (Grey and black bars indicate significantly regulated by any of the seven compounds in ESTn or ESTc, respectively).

genes were regulated up or down in one model by $\mathrm{MeHg}$ or FLU, FC regulation in the other model was either in the opposite direction or completely absent (Figure 4A, Figure 4C). VPA-regulated genes in general showed similar direction of gene expression in both models, in addition to genes significantly regulated in only one of the models (Figure 4E)

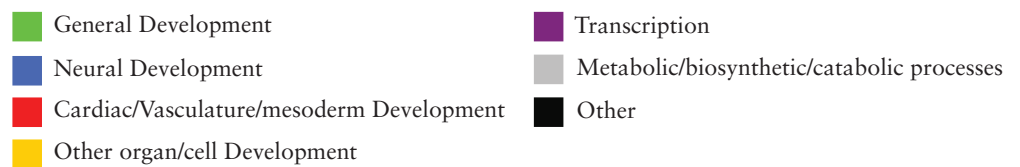

Figure 4 Compound related gene expression profiles in ESTn and ESTc. Hierarcha clustering of the genes regulated by $\mathrm{MeHg}(\mathrm{A}), \mathrm{FLU}(\mathrm{C})$ and VPA (E) in either ESTn and/or ESTc (FDR < 0.05). Hierarchal clustering of the genes regulated by VPA in either ESTn and/or ESTc $(F D R<0.05 ; \mathrm{FC}>1.1$, dark grey = significant in ESTn, black = significant in ESTc). Number of GOBP significantly enriched $(\mathrm{p}<0.001$, number genes regulated within GOBP > 5) after 24h exposure to B) $\mathrm{MeHg}$, D) FLU and F) VPA. Enrichment for each compound is shown based on all genes regulated, up-regulated genes and down-regulated genes; number of genes per group is indicated on top of each bar. GOBP function is depicted in each bar. 
$\mathrm{C}$

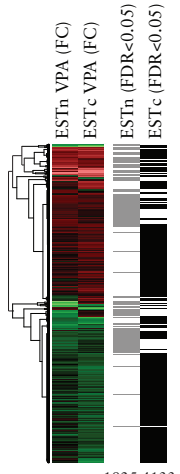

$\mathrm{F}$
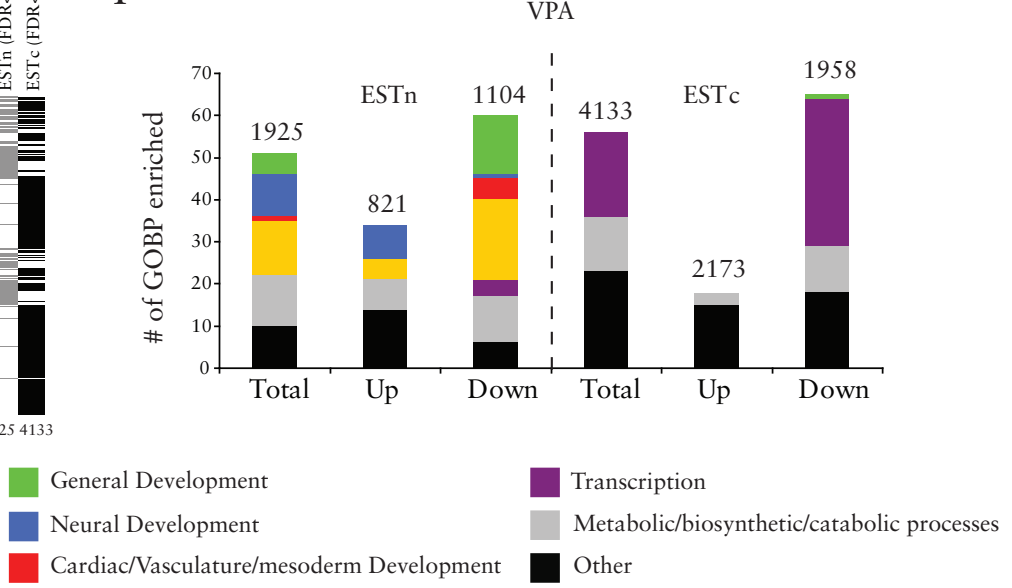

Other organ/cell Development

Figure 4 Continued.

G

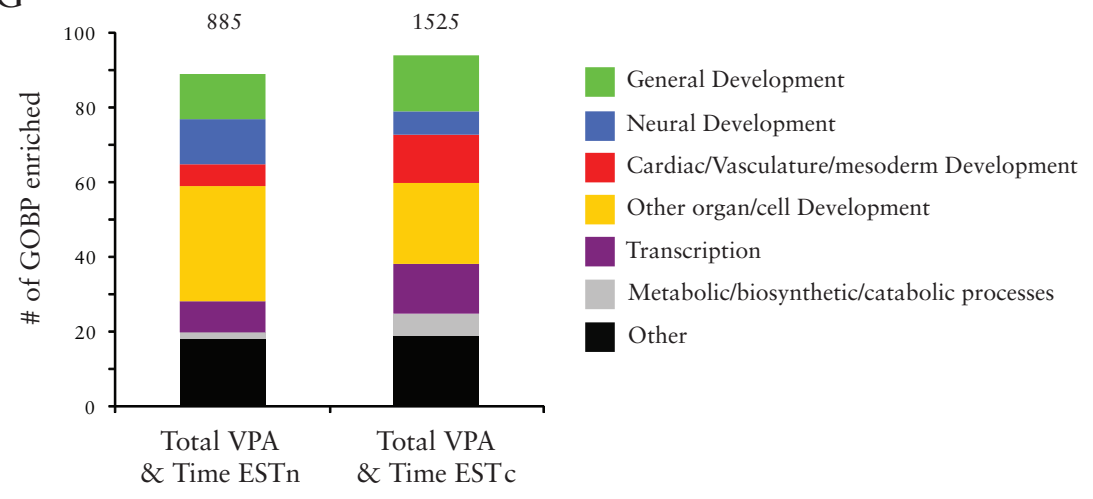

Figure 4 GOBP enriched by genes regulated both over time (FDR $<0.01 \mathrm{FC}>1.5)$ and by VPA (FDR $<0.05 \mathrm{p}<0.001$ ) in ESTn (885 genes) and ESTc (1525 genes). (GOBP $\mathrm{p}<0.001$, number genes regulated within GOBP $>5$ )

\section{Compound induced GOBP enrichment in ESTn and ESTc}

Analysis of GOBP enrichment by compounds and assays is shown in figure $4 \mathrm{~B}, 4 \mathrm{D}$ and $4 \mathrm{~F}$. In each figure the number of enriched GOBP is shown for all genes regulated by the compound, as well as separately for either upregulated or downregulated genes. Within each bar, a subdivision of GOBP in relation to specific themes is shown. Due to the statistical algorithms used for calculating enrichment, the number of enriched GOBP will depend on the number of genes used as input as well as the functional coherency of the input gene set. Therefore, the number of enriched GOBP can vary between the "larger" set of all regulated genes versus the "more focused" set of up- or downregulated genes.

In the ESTn, MeHg upregulated genes were specifically enriched for neural and cardiac development related GOBP and downregulated genes were enriched for general development GOBP (Figure 4B). In ESTc, MeHg upregulated genes were enriched for GOBP related to general development and other development (including mainly specific organ and cell differentiation GOBP) (Figure 4B). Furthermore in the ESTc, a large group of GOBP related to transcription and metabolism (macromolecule and nitrogen related) were enriched by $\mathrm{MeHg}$ upregulated genes.

GOBP enriched by FLU exposure in ESTn showed enrichment in both up- and down-regulated genes of general development related GOBP and other developmental GOBP (skeletal and epithelium development related GOBP (Figure 4D). Furthermore, FLU up-regulated GOBP involved in metabolism were related to sterol- and cholesterol metabolism, whereas downregulated GOBP were involved in glucose- and hexose metabolism. FLU exposure in ESTc had a large effect on development related GOBP, specifically cardiac, vasculature and mesenchyme related developmental processes, which were mainly enriched by down-regulated genes (Figure 4D). GOBP related to FLU up-regulated genes were involved in sterol- and cholesterol metabolism, whereas down-regulated genes were involved in transcription and metabolism related to transcription.

PA exposure in ESTn enriched many processes involved in development, including neural and cardiac development (Figure 4F). GOBP enriched by VPA up-regulated genes were involved in neural development, cellular morphogenesis related GOBP (other development) and transport related processes (other). GOBP enriched by VPA down-regulated genes were involved in general development, cardiac development, skeletal development ("other development" 
group) and cell proliferation ("other" group). Changes in gene expression induced by VPA in ESTc regulated processes involved in transcription and metabolic processes. GOBP enriched by VPA up-regulated genes were involved in cellular transport, whereas GOBP enriched by VPA down-regulated genes were involved in mitosis, stress, DNA repair, transcription and metabolic processes mainly involved in transcription. Transcription related term included a large group of GOBP related to epigenetics, including histone modification, methylation and chromosome reorganization.

In order to specifically investigate VPA effects on genes related to development, an additional gene selection was used by which genes had to be regulated both by VPA (FDR < 0.05) and over time (the 3648 genes defined earlier in Figure 1A). This resulted in 1599 genes (enriching 95 GOBP) and 885 genes (enriching 90 GOBP) regulated in ESTc and ESTn, respectively. Using this approach, for both models approximately 60 GOBP were involved in developmental related processes. In addition, more cardiac development and transcription related GOBP were enriched in ESTc whereas more neural- and "other" development related GOBP were enriched in ESTn (Figure 4G)

\section{\$ి}

\section{Discussion}

$\Gamma$ HE development and implementation of predictive alternative assays in developmental toxicology has been hampered by the complexity of embryo-fetal development. The multitude of mechanisms in development and their spatial and time-dependent relevance do not allow a single in vitro assay to contain all developmental mechanisms. Therefore, a combination of in vitro assays in a battery and/or tiered approach will be needed to achieve an acceptable level of prediction of in vivo developmental toxicity of tested chemicals. Various studies have addressed this issue, for instance by applying a group of available assays showing improved prediction of the combination over individual assays [175, 264], This study used a variety of assays based on receptor binding, cell differentiation, and whole embryo culture methods. The much larger ToxCast initiative used several hundreds of high throughput assays, primarily based on enzyme activity and receptor binding assays [265, 266]. This battery is being extended with more complex assays such as the EST and the zebrafish embryotoxicity assay to enhance predictivity [267, 268] Although it should be realized that the entire developmental programme is more than the sum of its constituents, a battery approach containing the most crucial mechanisms of development may be able to detect most embryotoxi- cants. In addition, such a battery should be used within an integrated testing strategy which also employs non-testing information such as physico-chemical properties and structure-activity relationships [11, 269].

The ESTc is one of the most extensively studied in vitro assays for developmental toxicity [18]. It has a rich history of validation efforts that have shown it possibilities and limitations $[17,36,37,264]$. One limitation relates to the readout of spontaneously contracting cardiomyocytes only. We hypothesized that the addition of alternative differentiation pathways might improve detection of developmental toxicants both in terms of numbers of compound detected as well as to mechanistic information gained about tested compounds. Therefore, we developed the ESTn as a complementary assay to the ESTc [142] We have performed a series of transcriptomics experiments in both models, studying time- and compound-perturbed gene expression effects, in order to enhance information about compound-mediated effects at the molecular level, and to possibly increase predictivity $[83,155,177,203,263]$. The basic difference between the ESTn and ESTc differentiation protocols in the curren observation period is the supplementation with RA. RA, an active metabolite of vitamin $\mathrm{A}$, is an essential regulator for instance in the physiology of vision and brain function, with blood serum levels in human adults of approximately $2 \mathrm{ng} / \mathrm{ml}$ (approximately $7 \mathrm{nM}$ ) [270]. RA is also known to play an essential role as a morphogen in normal embryogenesis. Subtle gradients of RA direct morphogenesis throughout the embryo, including limb development [271] and neural development $[272,273])$ through specific receptor interactions with the retinoic acid receptor family [274]. During early organogenesis in vivo, RA levels are observed to be highest in the neural tube [272]. This morphogen is employed to induce neural differentiation in ESTn. Other investigators have used similar or higher concentrations to induce neural differentiation in stem cell differentiation assays $[52,275]$. The extensive differences in differentiation pathways between the cardiac and neural differentiation assays is especially exemplified in the present study by the detailed analysis of clusters B and G, with development related signaling pathways (e.g. FGF, Wnt and BMP) and cardiac genes dominating in ESTc and homeobox gene and neural pathways dominating in ESTn (Figure 1). Reduced expression of cardiac developmen and Wnt- and TGF -signaling pathways by RA has been demonstrated before in differentiating mouse ESC in a comparable setting, where neural to mesodermal fate change occurred when RA signaling was inhibited, dependen on induction of Nodal-, Wnt- and FGF-signaling pathways [262]. Furthermore, in zebrafish it has been shown that early neural patterning is mediated by the Wnt-, FGF- and RA-signaling pathways. Wnt and FGF suppressed the Cyp26 
gene important in RA metabolism, whereas induced Cyp26 gene expression suppressed Wnt and FGF related genes [276].

The current comparison between existing data of ESTc and ESTn was performed using tested concentrations that were equipotent on the differentiation end point in the assays wherever possible. Therefore, the effective concentrations of a given compound analyzed might differ somewhat between tests, resulting in comparisons between different tested concentrations. However, given that the qualitative nature of the gene expression response was studied rather than its magnitude, such a comparison is justified. Differences in effective concentrations between assays were especially apparent with $\mathrm{MeHg}$, which showed a 20 -fold difference in ID50 between tests (Table 1). In addition, for FLU and HEX, cytotoxicity overruled effects on differentiation in ESTn but not ESTc. Therefore, from a pragmatic point of view, we decided to use IC20 concentrations in ESTn, which matched with ID50 concentration in ESTc and allowed useful comparisons of differentiation related gene expression signatures in both assays without domination of cell viability related genes only.

The added value of both tests combined over the use of a single assay was confirmed in the present study in several aspects. The well-known specific effect of $\mathrm{MeHg}$ on neurodevelopment [109] was mimicked both in its higher potency in ESTn as well as in the related gene expression pattern. For example, neurodevelopmental GOBP were uniquely affected in ESTn as compared to the more general transcription and metabolic processes but not cardiac GOBP dominating in ESTc. This is reminiscent of the reliable prediction of $\mathrm{MeHg}$ in ESTn as compared to its variable prediction in ESTc in the past $[17,155,277]$ As to FLU and HEX showing cytotoxicity at concentrations inhibiting neura differentiation in ESTn, the detailed analysis of FLU (Figure 4CD) indicates a similar effect on the gene expression level, with only few neural specific gene sets regulated in ESTn whereas in ESTc multiple cardiac gene sets appear affected.

VPA induced by far the highest number of genes among the compound analyzed in the present study. In both assays specific differentiation inhibition was observed in the absence of reduced cell viability. However, whereas in ESTn neural gene sets appeared to be regulated in the overall analysis as expected, ESTc failed to reveal effects on cardiac gene sets (Figure 4F). Restriction of the analysis of VPA induced gene expression changes to genes regulated during the differentiation process revealed both cardiac and neural gene sets regulated in both assays, with neural gene sets dominating in ESTn and cardiac gene sets dominating in ESTc (figure 4G). The relatively large number of genes regulated by this compound as compared to other tes compounds may have caused the less specific gene expression response observed. This analysis shows the advantage of restricting the gene expression analysis to the subset of genes that change their expression with the differen tiation process. These differentiation-related genes determine the "differentiation track" as defined in earlier studies [187]. This selection of genes can be used to reveal more specific information of compound effects on gene pathways related to differentiation in the assays.

In summary, we showed that combining ESTn and ESTc provided added value over individual assays by revealing complementary information on tes compounds. These observations serve to both improve the detection of differentiation-modulating compounds as well as show underlying gene expression changes indicating mechanisms of action. Such underlying knowledge will assist extrapolation from in vitro to in vivo as well as between species. The latter is ultimately important as the entire testing paradigm is focused toward risk assessment for man. The current interest in defining adverse outcome pathways (AOP) as the basis of hazard assessment $[226,278]$ is served by this approach in view of its detailed assessment of gene expression pathways underlying differentiation modulation. An inclusive combination of in vitro assays, each of which incorporating one of the critical AOP, could provide strong basis for a comprehensive pre-screen of in vitro prediction of human hazard. These findings exemplify the usefulness of combining complementary in vitro assays in in vitro tiered/battery approaches with the aim to improve overall detection of compounds potentially affecting embryo-fetal development.

\section{Lipereser}




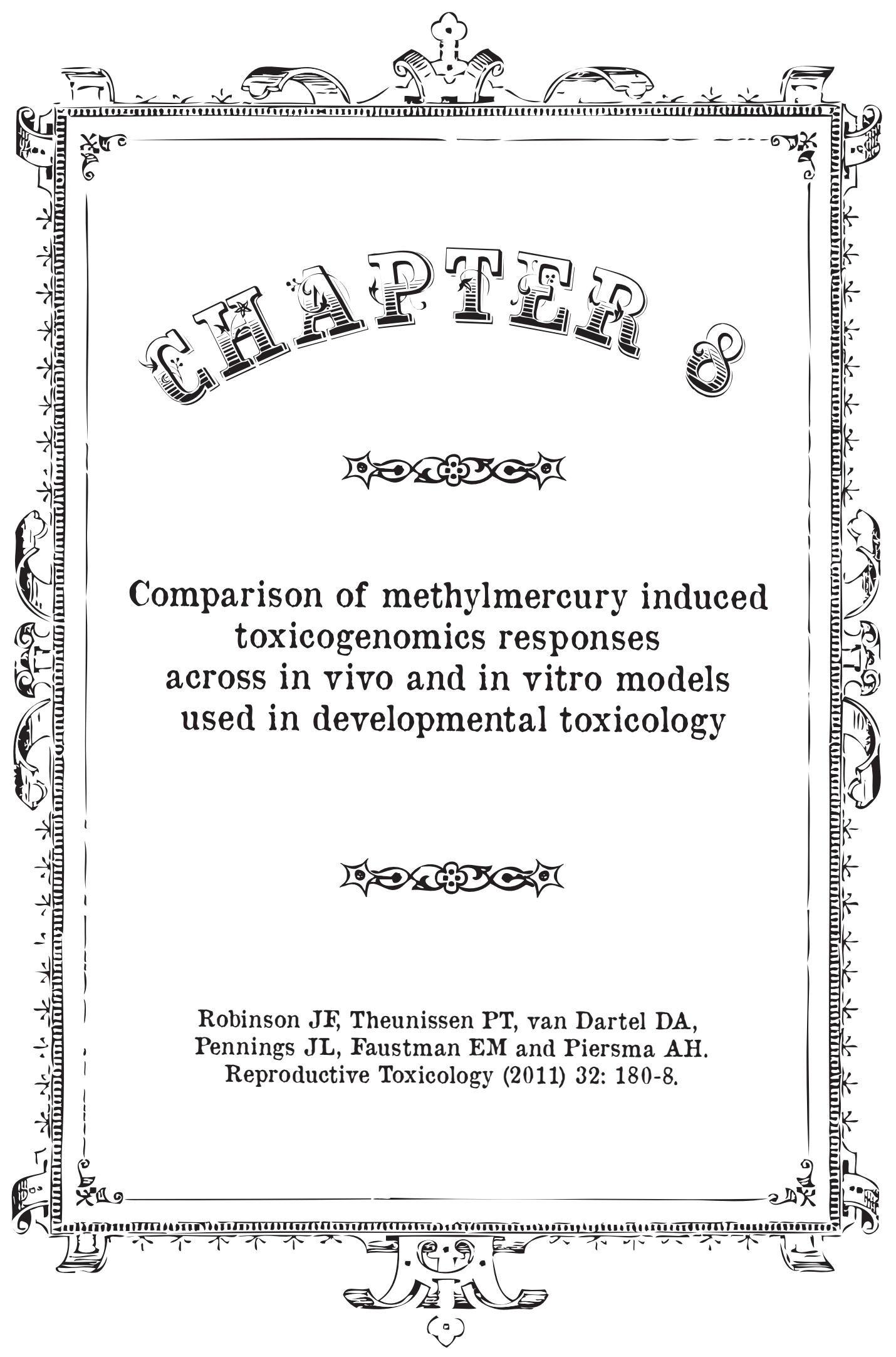




\section{Abstract}

OXICOGENOMIC evaluations may improve toxicity prediction of in vitroembryonic stem cells (ESC), by providing a robust mechanistic marker which can be linked with responses associated with developmental toxicity in vivo. While promising in theory, toxicogenomic comparisons between in vivo and in vitro models are complex due to inherent differences in model characteristics and experimental design. Determining factors which influence these global comparisons are critical in the identification of reliable mechanistic-based markers of developmental toxicity. In this study, we compared available toxicogenomic data assessing the impact of the known teratogen, methylmercury $(\mathrm{MeHg})$ across a diverse set of in vitro and in vivo models to investigate the impact of experimental variables (i.e. model, dose, time) on our comparative assessments. We evaluated common and unique aspects at both the functional (Gene Ontology) and gene level of MeHg-induced response. At the functional level, we observed stronger similarity in MeHg-response between mouse embryos exposed in utero (2 studies), ESC, and WEC as compared to liver, brain and mouse embryonic fibroblast $\mathrm{MeHg}$ studies. These findings were strongly correlated to the presence of a $\mathrm{MeHg}$-induced developmentally-related gene signature. In addition, we identified specific MeHg-induced gene expression alterations associated with developmental signaling and heart development across WEC, ESC and in vivo systems. However, the significance of overlap between studies was highly dependent on traditional experimental variables (i.e. dose, time). In summary, we identify promising examples of unique gene expression responses which show in vitro-in vivo similarities supporting the relevance of in vitro developmental models for predicting in vivo developmental toxicity.

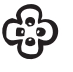

\section{Introduction}

UE to the implementation of the European REACH legislation for chemical safety and additional global pressures, a reduction in animal usage for toxicology studies is in high demand. The majority of toxicological testing is devoted to assess developmental health risks [12]. In vitro models, such as the rodent whole embryo culture (WEC) and embryonic stem cells (ESC), which can be induced into multiple lineages of cell types, such as neural (ESTn) and cardiomyocyte (ESTc), serve as potential replacements for current in vivo-based approaches to predict the potential for chemical compounds to induce developmental toxicity [175]. While less complex than whole animal models, in vitro developmental models possess characteristics (e.g. structure, mechanistic pathways) which are believed to allow partial representation of the whole organism developing in vivo. Multiple research groups are currently working on determining the applicability domain for these tests while trying to improve aspects of each methodology (e.g. duration, stability) to enable implementation into the risk assessment framework [14].

The integration of toxicogenomic evaluations may improve the prediction of developmental toxicity in vitro. Recent studies suggest toxicogenomics assessments assist in identifying 1) unique and common mechanisms of toxicity across multiple developmental toxicants [82, 155], 2) dose- [230, 279] and timeresponse [40,177], and 3 ) potential gene lists predictive of in vitro toxicity [83, 84, 187, 230]. Furthermore, in vitro toxicogenomic assessments provide mechanistic markers linked with developmental toxicity which can be identified across both in vivo and in vitro developmental models. Initial studies in either ESC or WEC evaluating compound effects of retinoic acid [280], methylmercury $[82,177]$, phthalates $[40,81,82]$ triazoles $[81,230]$ and glycol ethers [82 suggest specific transcriptomic responses to be relevant for developmental toxicity in vivo. These and future toxicogenomic studies conducted in developmentally-related models will produce valuable datasets to examine and link specific responses in vitro with in vivo developmental toxicity.

Although the identification of common responses across in vivo and in vitro models seems straightforward in principle, many factors exist in comparing across toxicogenomic studies due to inherent biological complexity of each model and each study. Recent gene expression studies completed in vivo and/ or in vitro suggest many factors to play a role in response, including mode [281], dose [152, 230], time [151, 282, 283], strain [151, 282], species [284, 285], gender [286], organ [287] and developmental time. As well, technical 
differences, such as RNA preparation, platform [68, 288], experimental practices [289], and differing statistical approaches may add obstacles in identifying common biomarkers across studies. In the area of carcinogenesis, initial comparative toxicogenomic assessments across multiple compounds have shown promise in identifying responses which distinguish genotoxicants from non-genotoxicants [290], however, these approaches may be too crude to examine the complexity of developmental toxicants due to vast mechanisms of toxicity and the specificity and comparability of common windows of sensitivity for particular toxicants. Initial comparisons examining responses across smaller sets of compounds and development models may assist in guiding which variables may factor in these comparative assessments.

Due to the public health relevancy surrounding exposure and inherent neurodevelopmental and teratogenic toxic properties [144], methylmercury ( $\mathrm{MeHg}$ ) is one of the most studied developmental toxicants. In the recent decade, toxicogenomic evaluations of $\mathrm{MeHg}$ have been conducted across a diverse set of conditions in both in vivo and in vitro developmental models. Therefore, using the unique availability of $\mathrm{MeHg}$ data, in this study, we investigated factors which influence comparisons of toxicogenomic response. We included currently available published datasets assessing $\mathrm{MeHg}$ in in vivo and in vitro models representative of early rodent embryonic development (five total) [82, $151,152,155,177]$. Also, we selected two in vivo studies assessing long term effects in brain [291] and kidney [287, 292] and one in vitro study (mouse embryonic fibroblasts) [292] for comparisons. We compared variables of interest, including model, dose, and time which may influence toxicogenomic comparisons across these studies which represented a variety of experimental conditions and toxicity outcomes. We conducted global comparative assessments across all eight studies, examining MeHg-induced functional changes (GO Biological Processes) as well as specific gene expression alterations associated with development via model by model comparisons between in vitro and in vivo developmental models. In general, these initial comparisons suggest that common toxicogenomic responses are able to be identified across in vitro and in vivo developmental models, however, many factors influence these comparative assessments. Moreover, this study provides insight into the specificity of models in response; specific developmental models may compliment other models better when assessing common toxicological responses across in vivo and in vitro models. These types of genomic based-analyses may guide in defining applicability domains of developmental models in terms of windows of sensitivity and chemical response.

\section{Methods}

\section{Acquisition of Datasets Associated with Gene Expression} Analysis of $\mathrm{MeHg}$ Effects

Retrieved toxicogenomic datasets regarding $\mathrm{MeHg}$ exposure were generated at different research institutions. Investigations concerning $\mathrm{MeHg}$ impacts in mouse embryos undergoing early gestational development [151,152] and mouse embryonic fibroblasts (MEF) [292] were performed at the University of Washington (US) under supervision by Dr. Elaine M. Faustman. Toxicogenomic studies regarding $\mathrm{MeHg}$ exposure in the WEC [82] and ESTn and ESTc system (neural and cardiomyocytes) $[155,177]$ were conducted at the National Institute for Public Health and the Environment, (The Netherlands) under supervision by Dr. Aldert H. Piersma. The dataset concerning $\mathrm{MeHg}$ gene expression alterations in mouse brain in gestational/postpartum-exposed mice [291] was accessed with permission from Dr. Chris N. Glover and Dr. Christer Hogstrand (National Institute of Nutrition and Seafood Research, Norway; University of Canterbury, New Zealand). The dataset investigating sub chronic $\mathrm{MeHg}$ effects in rat (kidney) was identified using ArrayExpress at EBI (E-TOXM-30, TNO Quality of Life, The Netherlands) [287].

All datasets were initially processed separately. Methodologies regarding statistical analysis of initial processing of each dataset were conducted as previously described when possible [82, 151, 152, 155, 177, 287, 291, 292]. A complete summary of processing steps (array type, normalization) are described in Supplementary Table 1 (see supplementary data original article), including modifications to previous methodologies. A general framework to investigate $\mathrm{MeHg}$-toxicogenomic responses across in vivo and in vitro datasets is illustrated in Supplemental Figure 1 (see supplementary data original article).

\section{Identification of Differentially Expressed Genes due to $\mathrm{MeHg}$}

\section{Exposure}

Normalized data were log transformed and imported into BRBarraytools (NCI) [293]. ANOVA was used to determine significantly altered genes by $\mathrm{MeHg}$ within each dataset across all experimental conditions (i.e. time, dose). P-values were corrected for multiple testing by calculating the False Discovery Rate (FDR) according to Benjamini and Hochberg [85]. All redundant probes (multiple probes for the same gene) within each study were eliminated; only the most significant ( $\mathrm{p}$-value) probe to be altered by $\mathrm{MeHg}$ was included for the following comparative analyses. 


\section{Functional Analysis of Differentially Expressed Genes across}

\section{Developmental Models}

Due to differences in distribution of significance and the complexity of using differing sizes of gene lists for later analyses, a ranking approach was implemented to make comparisons regarding common gene expression responses and assessing functional changes in gene expression (gene ontology analysis). The top 1000 genes were determined using ANOVA to develop a ranking value for each gene within each study. This approach translated into different criteria in terms of p-value cutoff for each one of the studies (see supplementary data original article, table 1). Enrichment of significantly enriched gene ontology (GO) Biological Processes was conducted using DAVID [294]. Enriched GO Biological Processes were determined to contain $\geq 5$ genes and a $\mathrm{p}<0.05$. Similar results were observed with assessments conducted using the top 500 (not shown) in terms of broad functional changes, however, less categories of genes were enriched. Hierarchical clustering (Manhattan, Average Linkage) was performed using the p-value associated with enrichment of GO Biological Processes across all eight studies to determine similarities in overrepresentation at the functional level for all significantly enriched GO Biological Processes and more specifically, developmentally-related terms (children terms of the GO Biological Process, developmental process) (TIGR MEV) [295, 296].

\section{Comparison of Developmental Genes Commonly Altered} between In Vivo and WEC

Due to common enrichment of $\mathrm{GO}$ developmentally-related processes in $\mathrm{MeHg}$ altered genes across multiple models and prior investigations suggesting these related genes to be critical for development of the organism, we further examined these related responses at the gene level to identify common biomarkers in the context of factors which influence our comparisons (model, dose, time). We assessed $\mathrm{MeHg}$ commonly altered genes related to the GO biological process, Developmental Process between mouse embryos [152] and rat WEC [82]. As described [152], the in vivo dataset used for these assessment investigated the impact of $\mathrm{MeHg}$ on gene expression in $\mathrm{C} 57$ mouse embryos (GD8 + 0, 8, 12h) across three dose groups $(1,4,6 \mathrm{mg} / \mathrm{kg} \mathrm{BW})$. The WEC study [82] examined gene expression changes at $4 \mathrm{~h}$ in $\mathrm{MeHg}$ exposed $(6 \mu \mathrm{g} / \mathrm{ml}$ embryos. Commonly represented genes in both studies (9166 genes) were determined using Official Gene Symbol (OGS) annotation. In total, 267 genes were identified to be significantly altered by $\mathrm{MeHg}$ in both studies irrespective of dose or time (ANOVA, $\mathrm{p}<0.05$ ). Employing DAVID, we identified $28 \%$ of these genes (76 genes) to be related to the GO biological process, developmental process. For these 76 genes, the relative fold change ( $\log 2$ scale) was determined comparing each dose/time group to its relative control using the average intensity. Cross-scatter plots were employed to visually compare the effect of $\mathrm{MeHg}$ across multiple conditions in vivo with WEC. Genes identified to be significantly altered in vivo at each particular condition (dose/time) were evaluated for significance using a post-hoc test (T-test, $\mathrm{p}<0.05$ ).

\section{Comparison of Developmental Genes Commonly Altered}

\section{between In Vivo and ESC systems}

Additionally, we assessed commonly MeHg-altered genes related to the GO biological process, developmental process between mouse whole embryos [152], ESTn [177] and ESTc [155]. As described above [152], the in vivo datase used for these assessments investigated the impact of $\mathrm{MeHg}$ on gene expression in C57 mouse embryos (GD8 + 0, 8, 12h) across three dose groups $(1,4,6 \mathrm{mg} /$ $\mathrm{kg} \mathrm{BW})$. The ESTn study [8] examined gene expression changes at 24, 48, 72 , $96 \mathrm{~h}$ in $\mathrm{MeHg}$ exposed $(25 \mathrm{nM})$ cultures. $\mathrm{MeHg}$-induced alterations were assessed in ESTc at 24h following $\mathrm{MeHg}$ exposure (480nM). Commonly represented genes across the three studies (17315 genes) were determined using Official Gene Symbol (OGS) annotation. Genes were identified to be significantly altered by $\mathrm{MeHg}$ across two of the three studies irrespective of dose or time (ANOVA, $\mathrm{p}<0.05$ ). Employing DAVID [294], we identified genes to be related to the GO Biological Process, developmental process. In total, 163, 281 and 181 developmentally-related genes were identified to be significantly altered between ESTn and ESTc; whole mouse embryos and ESTn; and whole mouse embryos and ESTn, respectively. The relative fold change $\left(\log _{2}\right.$ scale) was determined comparing each dose/time group to its relative control using the average intensity. Cross-scatter plots were created between all three studies. Selected comparisons are shown between the three model systems. Genes identified to be significantly altered in vivo/in vitro for each particular condition (dose/time) were evaluated for significance using a post-hoc test (T-test, $\mathrm{p}<0.05)$, when necessary.

\section{Overlapping $\mathrm{MeHg}$-induced Alterations in Developmental In Vivo / In Vitro models}

To investigate the overlap in $\mathrm{MeHg}$ response between developmental in vitro and in vivo models, we identified genes commonly significantly altered (ANOVA, $\mathrm{p}<0.05$ ) in at least four of the five available studies [82, 151, 152, $155,177]$. Datasets were merged by OGS annotation. Fold change values for all 53 genes identified to be significantly altered in four of the five studies (ANOVA, $\mathrm{p}<0.05$ ) were examined for response to $\mathrm{MeHg}$ (in comparison with comparative control) using hierarchical clustering (TIGR MEV, mean average). 
Classification of developmental- and apoptosis-related genes was conducted using DAVID. In addition, genes previously identified to be critical for neurulation in mouse models [297, 298] were labeled.

\section{母}

\section{Results}

\section{Microarray Datasets Investigating MeHg-induced}

\section{Developmental Toxicity}

We examined the comparative relationship of $\mathrm{MeHg}$-induced gene expression response across eight transcriptomics studies (labeled A-H) (Table 1). Specifically, we obtained four in vivo studies assessing $\mathrm{MeHg}$ effects on gene expression in mouse embryos exposed in utero (A, B), young mice (brain) exposed in utero and during postnatal period (C) and adult rats (kidney) chronically exposed to $\mathrm{MeHg}(\mathrm{D})$. We compared responses in these in vivo models with studies conducted in four alternative (in vitro)-based assays WEC (E), ESTn $(F), \operatorname{ESTc}(\mathrm{G})$, and MEF $(\mathrm{H})$. Different parameters were observed across all eight studies regarding, model, dose, time (exposure duration), and linked toxicity endpoint.

\section{Overlapping Enrichment of MeHg-induced Impacts across In Vivo and In Vitro Developmental Models}

As shown in figure 1A, we identified overlapping and non-overlapping enrichment of GO Biological Processes within the most prominent MeHg-induced gene expression alterations (top 1000 in each dataset) across the eight datasets examined. In total, $537 \mathrm{GO}$ Biological Processes were identified to be significantly enriched in at least one of the eight datasets ( $\geq 5$ genes changed, $p$ $<0.05$ ). Twenty-five percent of enriched GO Biological Processes were related to Developmental Process terms (Figure 1B). Other general themes previously linked with MeHg-toxicity were identified such as Cell Differentiation (6\%) and Stress-related $(6 \%)$ (e.g. cell death, cell cycle arrest, oxidative stress, DNA damage). Hierarchical clustering of the degree of enrichment ( $p$-value) of the 537 GO Biological Processes indicated similarity between datasets A and B (mouse embryos) and in vitro models (E, F, G) as compared to datasets C, D, and $\mathrm{H}$ (Figure 1C).

Overlap in enrichment of developmentally-specific GO Biological Processes were apparent between datasets, in particular, between A, B, E, F, and $\mathrm{G}$ (Figure 2A). Hierarchical clustering of the degree of enrichment ( $p$-value) of
Table 1 In Vivo and In Vitro Study Comparisons: MeHg-induced Toxicogenomic Changes in Gene Expression

\begin{tabular}{|c|c|c|c|c|c|c|}
\hline$\frac{\pi}{8}$ & $\begin{array}{l}\frac{\mathscr{U}}{\tilde{U}^{\prime}} \\
\hat{\nu}^{2}\end{array}$ & $\frac{\bar{v}}{\sum_{0}^{\circ}}$ & 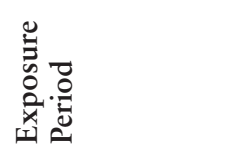 & 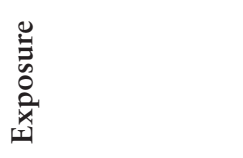 & 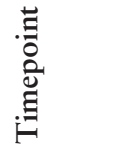 & : \\
\hline A & $\mathrm{M}$ & Embryo & $\begin{array}{l}\text { Neurulation } \\
\text { Early } \\
\text { Organogenesis }\end{array}$ & $\begin{array}{l}\text { 1, 4, 6mg/kg (ip), } \\
\text { GD8 }\end{array}$ & $8,12 \mathrm{~h}$ & $\begin{array}{l}\text { BW, growth, } \\
\text { reproductive, } \\
\text { structural defects } \\
(\geq 4 \mathrm{mg} / \mathrm{kg})\end{array}$ \\
\hline B & $\mathrm{M}$ & Embryo & $\begin{array}{l}\text { Neurulation } \\
\text { Early } \\
\text { Organogenesis }\end{array}$ & $\begin{array}{l}\text { 4mg/kg (ip), } \\
\text { GD8 }\end{array}$ & $12,24 \mathrm{~h}$ & $\begin{array}{l}\text { Neural tube closure } \\
\text { delay reproductive } \\
\text { and growth effects }\end{array}$ \\
\hline $\mathrm{C}$ & M & Brain & $\begin{array}{l}\text { Gestation } \\
\text { Post-partum }\end{array}$ & $\begin{array}{l}1.5,4.5 \mathrm{mg} / \mathrm{kg} \\
\text { (food) } \\
\text { (11 weeks) }\end{array}$ & $24 \mathrm{~h}$ & $\begin{array}{l}\text { Impaired brain } \\
\text { function, resorptions } \\
(\geq 1.5 \mathrm{mg} / \mathrm{kg})\end{array}$ \\
\hline D & $\mathrm{R}$ & Kidney & Adult & $\begin{array}{l}0.25,0.75 \mathrm{mg} / \mathrm{kg} \\
\text { (gavage) } \\
\text { (14 days) }\end{array}$ & NA & NOAEL $(0.75 \mathrm{mg} / \mathrm{kg})$ \\
\hline $\mathrm{E}$ & $\mathrm{R}$ & Embryo & $\begin{array}{l}\text { Neurulation } \\
\text { Early } \\
\text { Organogenesis }\end{array}$ & $\begin{array}{l}6 \mu \mathrm{g} / \mathrm{mL} \\
(\mathrm{GD} 10)\end{array}$ & $4 \mathrm{~h}$ & $\begin{array}{l}\text { Reduction in total } \\
\text { morphological score }\end{array}$ \\
\hline $\mathrm{F}$ & $\mathrm{M}$ & ESCn & $\begin{array}{l}\text { Neural } \\
\text { Differentiation }\end{array}$ & $\begin{array}{l}25 \mathrm{nM} \\
(\mathrm{d} 3)\end{array}$ & $\begin{array}{l}24,48 \\
72,96 h\end{array}$ & $\begin{array}{l}\text { Inhibition of } \\
\text { differentiation }\end{array}$ \\
\hline G & $\mathrm{M}$ & ESCc & $\begin{array}{l}\text { Cardiomyocyte } \\
\text { Differentiation }\end{array}$ & $\begin{array}{l}480 \mathrm{nM} \\
\text { (d3) }\end{array}$ & $24 \mathrm{~h}$ & $\begin{array}{l}\text { Inhibition of } \\
\text { differentiation }\end{array}$ \\
\hline $\mathrm{H}$ & $\mathrm{M}$ & MEFs & Normal Culture & $5 \mathrm{mM}$ & $24 \mathrm{~h}$ & Increased cell death \\
\hline
\end{tabular}

Coding (A-H) signifies study label throughout the manuscript and for shorthand notation of select figures. Toxicogenomic studies were completed in rat $(\mathrm{R})$ or mouse $(\mathrm{M})$.

the 136 developmentally-related GO Biological Processes suggested greater similarity in functional response between datasets, $\mathrm{A}$ and $\mathrm{E}$ and $\mathrm{B}, \mathrm{F}, \mathrm{G}$ as compared to $\mathrm{C}, \mathrm{D}$ and $\mathrm{H}$ (Figure $2 \mathrm{~B}$ ). A selection of specific GO Biological Processes associated with Developmental Process, Heart Development, Anterior/Posterior Pattern Formation, and Regulation of Neurogenesis were significantly enriched $(\mathrm{p}<0.05)$, dependent on model (Figure $2 \mathrm{C})$. For example, we observed enrichment of the most general developmentally-related GO Biological Process, Developmental Process, across models, A, B, E, F, G. Enrichment of genes related to Heart Development were only observed in models A, B, E and G. 
A

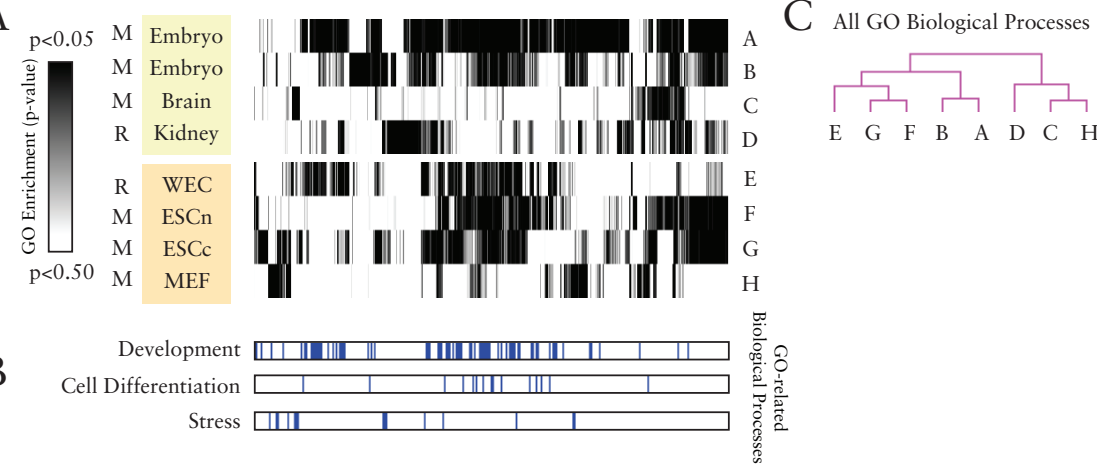

Figure 1 GO Enrichment of MeHg-induced Gene Expression Alterations across In Vivo and In Vitro Models. Enrichment of GO Biological Processes within the most prominent $\mathrm{MeHg}$-induced responses (top 1000 genes in each study) across eight in vitro and in vivo studies (Figure 1A). Species (mouse or rat) and examined tissue/cell type are located to the left of the hierarchical clustering plot depicting the enrichment score (p-value) of GO biological processes identified using DAVID. In total, 537 GO Biological Processes identified to be significantly enriched in one of the eight datasets $(p<0.05$, gene changed $>5$ ) are depicted by color intensity (columns). GO Biological Processes related to development, cell differentiation and stress are labeled by color bar corresponding with the columns above (Figure 1B). Hierarchical clustering by study using the enrichment score (p-value) of all 537 GO Biological Processes (Figure 1C). Coding (A-H) corresponds with coding in Figure 1A.

MeHg-induced Developmental Gene Expression Alterations in Mouse Embryo and WEC: The influence of dose and time

Developmental genes commonly significantly altered by $\mathrm{MeHg}$ (76 total) in mouse embryo (A) and WEC (E) were examined for comparative expression changes between models in association with dose- and time-dependent effects (in vivo) (Figure 3). In general, we observed an overrepresentation of common up-regulated gene expression responses (quadrant I) in the 76 genes related to development between both studies across all conditions in vivo (Figure $3 \mathrm{~B}$, a-e). We observed a dose-dependent effect in the number of genes commonly altered between studies with increasing dose (in vivo) in our $8 \mathrm{~h}(\mathrm{a}-\mathrm{c})$ and $12 \mathrm{~h}$ (d, e) comparisons with the WEC (Figure 3B). For example, 54/76 genes were significantly altered between in vivo $(6 \mathrm{mg} / \mathrm{kg}, 8 \mathrm{~h})$ and WEC $(6 \mu \mathrm{gg} / \mathrm{ml}, 4 \mathrm{~h})$. Seventy-four percent of the 54 genes were observed to be commonly upregulated (quadrant I) in this comparison. Common directionality was observed for $>60 \%$ of all commonly altered genes dependent on comparison. Up-regulation of specific targets was observed for genes such as Celsr1, Chd7, Frem2, Cdx2, and Smarcc1 in both models (Figure 3C).
A
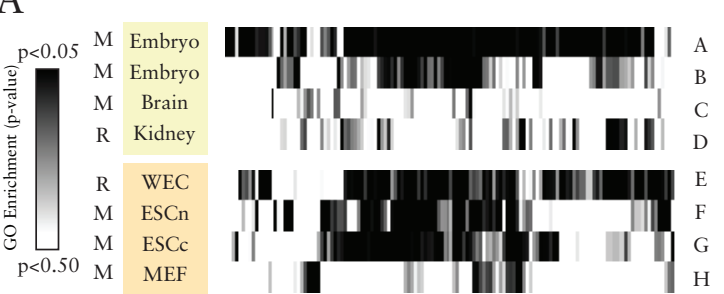

B Developmental GO Biological Processes A E F G

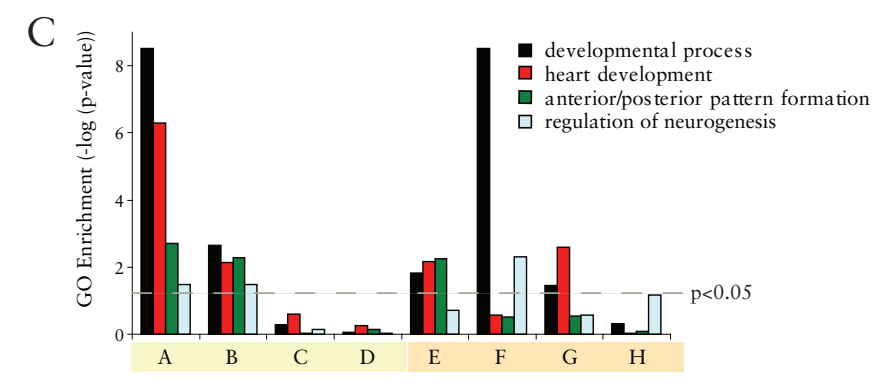

Figure 2 Enrichment of Developmentally-related GO Biological Processes across In Vivo and In Vitro Models. Enrichment of GO Biological Processes related to development within the most prominent MeHg-induced responses (top 1000 genes in each study) across eight in vitro and in vivo studies (Figure 2A). Species (mouse or rat) and examined tissue/cell type are located to the left of the hierarchical clustering plot depicting the enrichment score ( $\mathrm{p}$-value) of GO Biological Processes identified using DAVID. In total, 157 GO Biological Processes related to development identified to be significantly enriched in one of the eight datasets $(p<0.05$, genes changed $>5)$ are depicted by color intensity (columns). Hierarchical clustering by study using the enrichment score (p-value) of all 157 GO Biological Processes (Figure 2B). Examples of model dependent enrichment of select developmentally-related GO Biological Processes (Figure 2C).

Comparison of MeHg-induced Developmental Gene Expression Alterations in the ESC Model and Mouse Embryo

In comparisons between models A (mouse embryo), F (ESTn) and G (ESTc), we observed 165 (a), 281 (b), 181 (c) genes commonly altered between G and F; F and $\mathrm{A}$; and $\mathrm{G}$ and $\mathrm{A}$, respectively (Figure $4 \mathrm{~A}$ ). Comparing across all three studies, 67 genes were identified to be commonly significantly altered by $\mathrm{MeHg}$ in all three models (Figure 4A)

In figure 4Ca, 165 genes identified to be significantly altered in both ESTn and ESTc model systems are plotted using a cross-scatter plot. At $24 \mathrm{~h}$, in both ESTc and ESTn, 84 genes (blue triangles) were identified to be commonly 
A

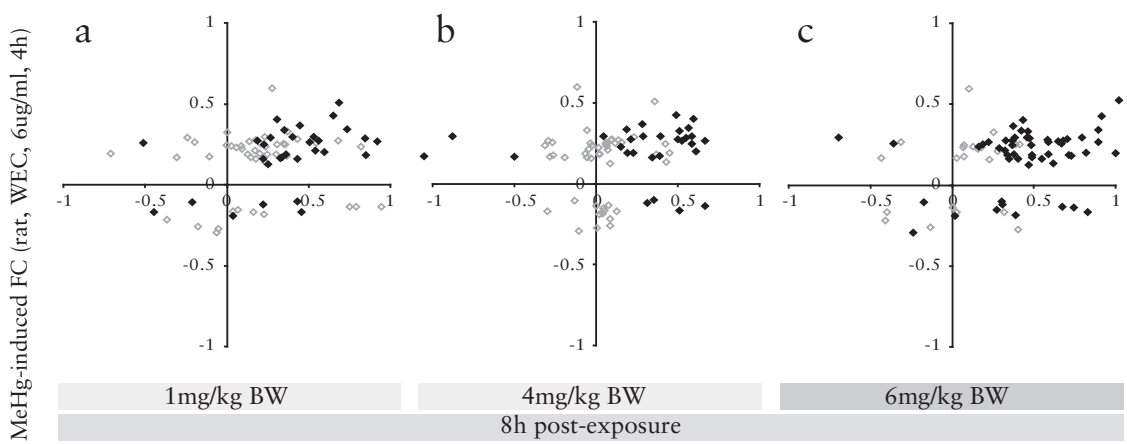

MeHg-induced FC (mouse, in vivo, GD8 exposure)

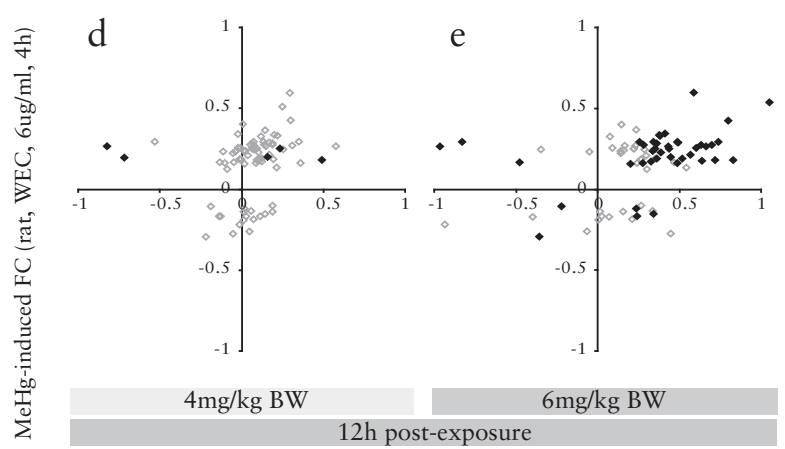

MeHg-induced FC (mouse, in vivo, GD8 exposure)

Figure 3 MeHg-induced Developmental Gene Expression Alterations in Mouse Embryo and WEC. Common developmentally-related MeHg-induced gene expressio alterations in in vivo (whole mouse embryo) and WEC models across different dose and time conditions (in vivo) (Figure 3A, a-e). In total, 76 genes were identified to be commonly altered across all conditions tested in the two studies. Post-hoc analysis (T-test) was used to determine significance in particular dose/time groups $(\mathrm{p}<0.05$, dark diamonds). Figure 3B describes relationships observed in Figure 3A. The percentage of the 76 genes showing common directionality between the two models in the degree of response ( $\%$ common direction). The percentage of the 76 genes identified to be commonly upregulated (Quadrant I) (\% common direction, Quadrant I). The total number of commonly altered developmentally-related genes, significant in both models in particular conditions (\# commonly altered). The percentage of commonly altered genes showing similar directionality between the two models in the degree of response (\% common direction). The percentage of the commonly altered genes identified to be commonly upregulated (Quadrant I). Comparison of fold change response in $\mathrm{MeHg}$-induced developmentally-related gene expression alterations in mouse embryos (GD8, $6 \mathrm{mg} / \mathrm{kg}, 8 \mathrm{~h}$ ) and rat WEC (GD10, $6 \mathrm{\mu g} / \mathrm{ml}$, 4h) (Figure 3C).

\begin{tabular}{|l|c|c|c|c|c|c|}
\hline Comparison & Key & a & b & c & d & e \\
\hline$\%$ common direction $(76$ genes) & $\searrow$ & $76 \%$ & $62 \%$ & $78 \%$ & $67 \%$ & $78 \%$ \\
\hline$\%$ common direction Quadrant I & & $68 \%$ & $58 \%$ & $71 \%$ & $59 \%$ & $71 \%$ \\
\hline \#commonly altered & & 31 & 29 & 54 & 5 & 42 \\
\hline$\%$ common direction (com alt genes) & & $81 \%$ & $76 \%$ & $78 \%$ & $60 \%$ & $83 \%$ \\
\hline$\%$ common direction Quadrant I & $744 \%$ & $76 \%$ & $74 \%$ & $60 \%$ & $79 \%$ \\
\hline
\end{tabular}

76 senes relot to der

76 genes related to development commonly significantly alterd
in vivo and in vitro (WEC) across all conditions within study

C

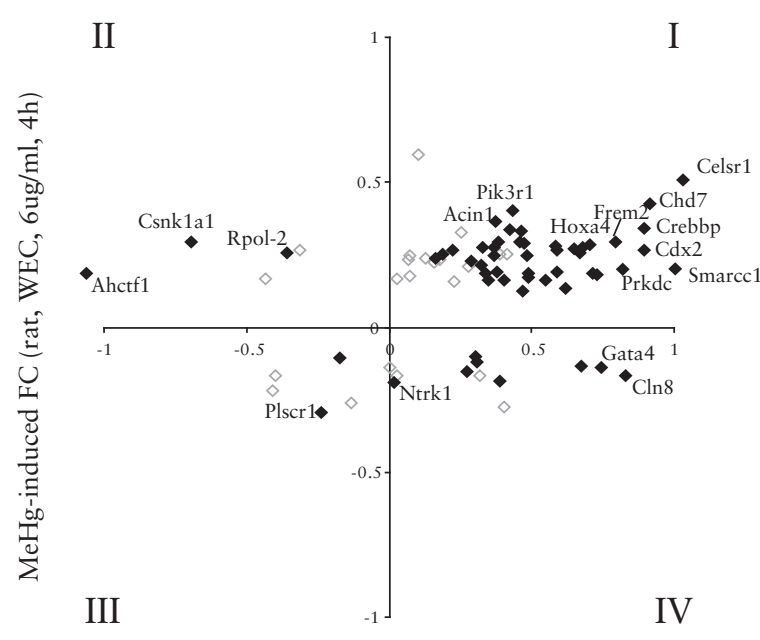

MeHg-induced FC (mouse, in vivo, $6 \mathrm{mg} / \mathrm{kg}$ BW $(8 \mathrm{~h})$ )

Figure 3 Continued

disrupted in expression (Figure 4B). Within these 84 genes, 56\% showed common directionality (Quadrant I and III). Common alterations include genes such as Gata3 $(\uparrow)$ and Ndrg2 ( $\downarrow)$. In general, strong correlation in the directionality of response of all significantly altered genes was not observed between the two systems.

In ESTn (24h, $25 \mathrm{nM})$ and mouse embryos $8 \mathrm{~h}(6 \mathrm{mg} / \mathrm{kg}), 83 / 281$ altered genes, showed common significance in this particular comparison (Figure $4 \mathrm{Cb}$ ). O the 83 genes, $45 \%$ were observed to be altered in the same direction, including

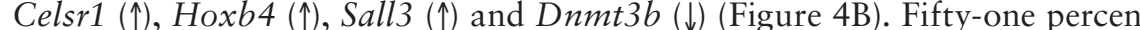
of the 83 genes were specifically down-regulated in mouse embryos and up-regulated in the ESTn (quadrant III). 
In comparisons between ESTc $(24 \mathrm{~h}, 480 \mathrm{nM})$ and mouse embryos $(6 \mathrm{mg} / \mathrm{kg}$, $8 \mathrm{~h}), 102 / 181$ genes were identified to be significantly altered (Figure 4Cc). Sixty one percent of the 102 genes showed common up/down-regulation (Tgfb2, Tbx3, Msx1, Gata3) between the two models (Figure 4B). Improved correlation in terms of common directionality of response $(86 \%)$ was observed within the subset of developmental genes related to heart development (Figure 4Cd). Commonly upregulated genes related to heart development included Tbx3, Tgfb2, Msx1, Tbx20, Nrp1, and Nrp2.

\section{Biomarkers of $\mathrm{MeHg}$ developmental toxicity applicable across}

\section{developmental in vitro and in vivo models}

Overlapping $\mathrm{MeHg}$-induced gene expression alterations were determined across developmental models (A, B, E, F and G). In total, 53 genes were identified to be altered by $\mathrm{MeHg}$ (ANOVA, $\mathrm{p}<0.05$ ) in 4/5 studies (Figure 5). Enrichment of developmentally- and cell death-related GO Biological Processes was observed within the 53 genes (data not shown). In total, 34\% (18 genes) of the 53 genes were identified to be developmentally related, including five previously identified as neural tube defect (NTD) candidate genes in mouse models (i.e. Frem2, Gja1, Piga, Cycs, Apaf1).

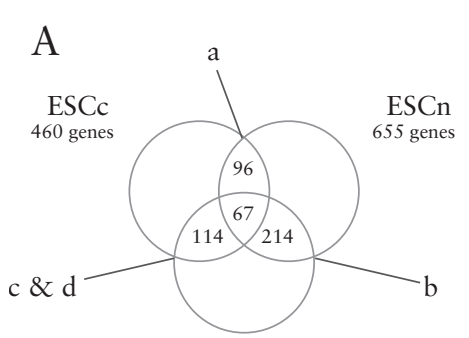

B $\underset{900 \text { genes }}{\text { Embryo (in vivo) }}$

C

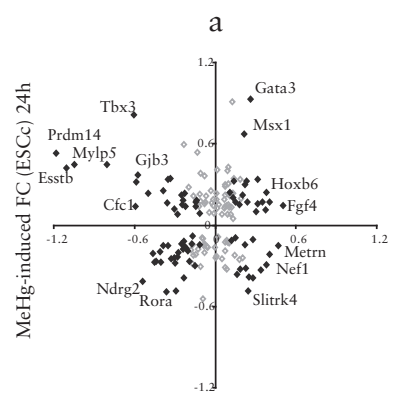

MeHg-induced FC (ESCn) 24h

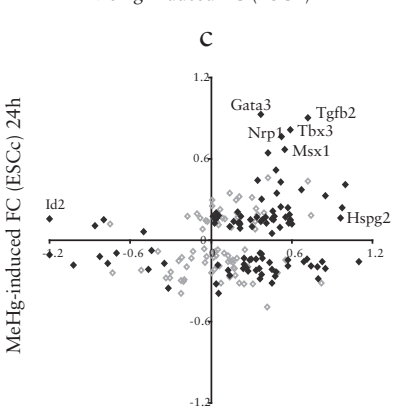

MeHg-induced FC (Embryo (in vivo)) 8h - Significantly altered in both models for particular condition $(\mathrm{p}<0.05)$

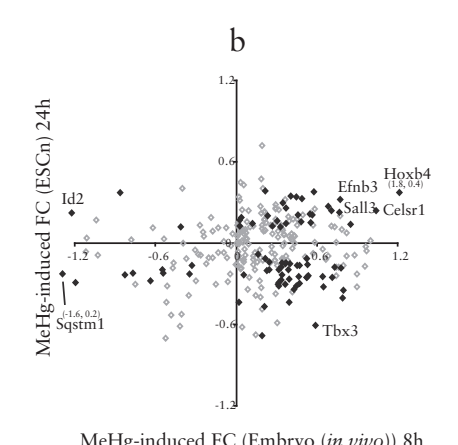

MeHg-induced FC (Embryo (in vivo)) 8h

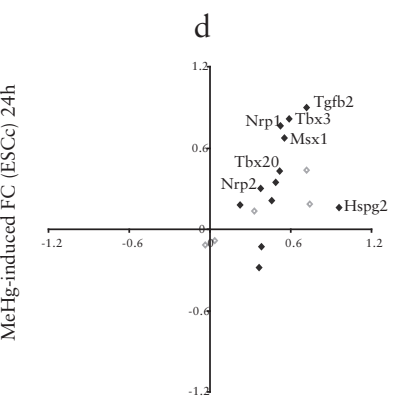

eHg-induced FC (Embryo (in vivo)) 8h

lar condition $(\mathrm{p}<0.05$

Figure 4 MeHg-induced Developmental Gene Expression Alterations in Mouse Embryo and ESC Model Systems. The overlap in significant developmentally-related gene expression alterations in MeHg exposed mouse embryos, ESTn and ESTc (Figure 4A). Figure 4B describes relationships observed in Figure 4C. Cross scatter plots comparing common MeHg-developmentally related gene expression alterations in ESTn and ESTc (Figure 4Ca), mouse embryo and ESTn (Figure 4Cb), and mouse embryo and elated gene expression a exlated gene expression alterations in mouse entho analysis (T-test) was used to determine significance in particular dose/time groups ( $\mathrm{p}<$ 0.05 , dark diamonds) 
Average fold change $(\mathrm{MeHg} / \mathrm{Con})$ for each gene

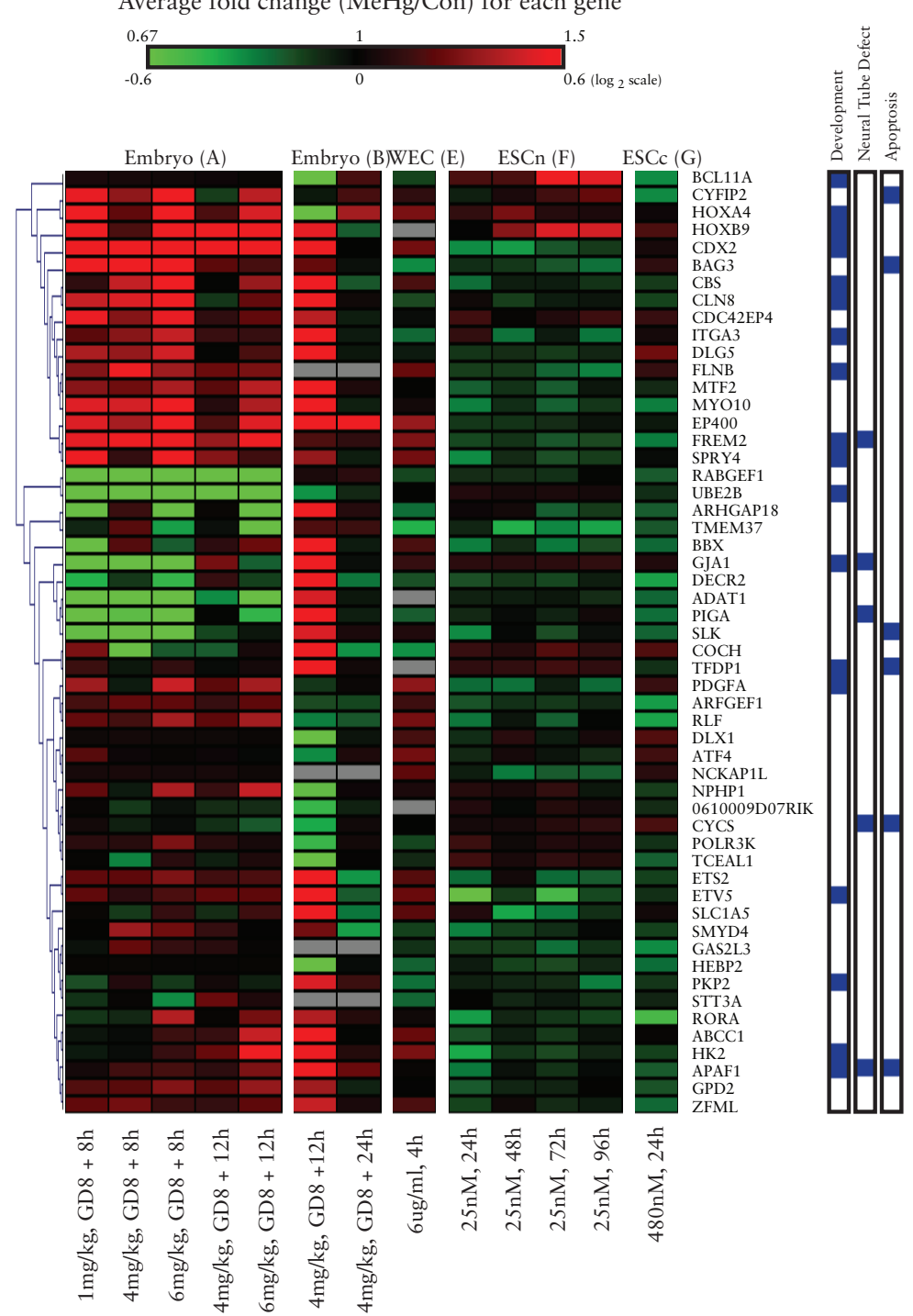

Figure 5 Overlapping MeHg-induced Transcriptional Alterations across Developmental Models. Hierarchical clustering of all 53 genes identified to be altered by $\mathrm{MeHg}$ in $4 / 5$ developmental in vivo/in vitro models (A, B, E, F, G) (ANOVA, $\mathrm{p}<0.05)$. Fold change values ( $\log$ ratios) are presented in color Genes related to GO Biological Processes, developmental process and apoptosis are labeled (right). In addition, genes previously identified to be linked with neural tube defects in mouse models are labeled (right).

\section{Discussion}

A Lternative models, such as the WEC and ESC systems are being developed A as potential replacements for in vivo-based approaches to determine the potential for chemicals to induce developmental toxicity [14]. Established protocols for these alternative models use morphological criteria to classify chemical toxic properties $[142,299]$. The integration of toxicogenomic evaluations may improve the capability of in vitro-based models by providing robust mechanistic markers used for classification and prediction [187, 280, 300]. Toxicogenomic responses identified in vitro provide a subjective endpoint which can be correlated with responses across other models, in particular, in vivo models assessing the relationship between exposure and developmental disease. Therefore, toxicogenomic evaluations enable a linkage between in vitro and developmental toxicity outcomes in vivo. In theory, the identification of these biomarkers or "phenotypic anchors" associated with developmental toxicity in vivo that are also identified to be altered in vitro due to chemical exposure should provide a more reliable, mechanistic-based objective endpoin to classify and predict developmental toxicity potential. The comparability of toxicogenomic data across models may also provide a robust measure to determine the applicability domain of a particular test in terms of chemical, organ and developmental window specificity. Due to the complexity of the developing organism and the wide diversity of mechanisms of developmental toxicants, the generation of toxicogenomic data assessing multiple variables is crucial in understanding the potential use of toxicogenomic data in making these types of comparisons across in vitro and in vivo developmental models.

Toxicogenomic comparisons across studies are complex because of inherent differences in experimental design and model. Determining factors which influence these global comparisons are critical in the identification of reliable mechanistic based markers of developmental toxicity. Here, as a proof of principal, we examined eight in vivo and in vitro datasets examining the mpact of the known developmental neurotoxicant and teratogen, $\mathrm{MeHg}$, across a wide set of diverse study designs (Table 1). MeHg-induced gene expression alterations across the eight studies were assessed from the functional and gene level. At the functional level (GO biological process), we provide evidence of specific in vitro models to share response similarities with early developmental models (i.e. neurulation, mouse) as we observe better correlations with enrichment of $\mathrm{MeHg}-\mathrm{GO}$ responses as compared to other study designs in vivo (kidney, brain) and in vitro (MEFs) (Figure 1). Differences at the functional level between early developmental models (A, B, E, F, G) and 
chronic organ models $(\mathrm{C}, \mathrm{D})$ /cultured $\mathrm{MEFs}(\mathrm{H})$ included the presence (or absence) of a robust $\mathrm{MeHg}$-induced developmental-gene signature (Figure 2A, B). Furthermore, gene categories (GO Biological Processes) related to specific processes were only observed in select models (Figure 2C). For example, while the most general developmental term, developmental process, was observed to be enriched across models A, B, E, F, G, children terms of the parent term developmental process were also only observed to be enriched in particular models. While we hesitate to make strong conclusions without further analyses and more representative datasets assessing across dose and time for the selected study designs, our results give a preliminary assessment of the comparative signature specificity across these particular in vivo and in vitro models and highlight the specificity of select in vitro models to present (functionally) similar gene expression signatures as early developing embryos exposed to $\mathrm{MeHg}$.

Due to the specificity of developmental signatures from the functional level in select developmental models (A, B, E, F, G), we assessed whether commonly altered developmentally-related genes were regulated similarly between these models in terms of significance and directionality. Moreover, we examined differences in our comparative assessments due to factors which influence the comparability of response, including model, dose and time.

In comparisons between mouse embryos (A) and WEC (E) we observed correlations (in terms of common directionality) in gene expression response influenced by dose and time (in vivo). Better correlations (\# of genes commonly altered, directionality), in general, were observed with the highest dose tested $(6 \mathrm{mg} / \mathrm{kg}, 8 \mathrm{~h}$ and $12 \mathrm{~h})$ in the mouse embryo study (A) and WEC $(6 \mu \mathrm{g} / \mathrm{ml}$, $24 \mathrm{~h}$ ), including multiple genes critical for embryonic development (e.g. Celsr1, Chd7) [301, 302]. These simple comparisons indicate the complexity of aligning in vitro and in vivo responses. Careful consideration must be assessed for dose and time-dependent factors when determining if an overlap in response exists between two systems. In these comparisons, dose levels $(\geq 4 \mathrm{mg} / \mathrm{kg}$ ) of $\mathrm{MeHg}$ in utero and the concentration used in the WEC $(6 \mu \mathrm{g} / \mathrm{ml}$ ) are comparable in their ability to induce teratogenic effects, including effects on neural tube closure and reduced growth (i.e. body weight, somites, size) [82, 151, 152, 173, 303]. While these models hold inherent similarities in terms of the exposure window $\left(G D 8_{\text {mouse }} \approx G D 10_{\text {rat }}\right)$, multiple differences in study designs could explain differences in observed responses, including, species differences, maternal environment versus culturing conditions, concentration, time point, $\mathrm{MeHg}$-kinetics, probe specificity, and other experimental considerations.
In the two ESC systems (F, G), at the functional level, we observed strong similarities in MeHg-response for enriched GO Biological Processes as well as developmentally specific categories (Figure 1,2). Despite these observations, only $56 \%$ of commonly altered developmental genes were observed to correlate in the same direction in response to $\mathrm{MeHg}$ in the two systems at $24 \mathrm{~h}$ following day 3 of culture $(\mathrm{p}<0.05)$ (Figure 4Ca). Interestingly, the ESC models were derived from the same blastocyst-derived cell line (ES-D3, ATCC) and studie were conducted in the same laboratory suggesting possible similarities. However, stark differences in culturing procedures between the ESTn and ESTc occur after culture day 3 with the addition of growth factors (e.g. retinoic acid on day 3) which induce neural versus cardiomyocyte differentiation [40, 142]. Our results support commonalities in $\mathrm{MeHg}$ effects between the two systems at the gene level (e.g. Msx1, Ndrg2), but also point out major difference in terms of the directionality of response (e.g. Tbx3, Prdm14) in genes significantly disrupted in both systems $(\mathrm{p}<0.05)$. Differences in response between these two studies may be explained by the addition of retinoic acid on day 3 of culturing, as well as differing concentrations (ESTn, $25 \mathrm{nM}$ and ESTc, $480 \mathrm{nM}$ ) and sensitivity of the two systems (neural more sensitive on a concentration basis).

As observed in comparisons between the WEC and mouse embryo (A) study, we observed similarities in $\mathrm{MeHg}$ response at the functional level (Figure 2) for developmentally-related GO Biological Processes between both ESC system $(\mathrm{F}, \mathrm{G})$ and mouse embryos (A, B). Cross comparisons of commonly altered developmental genes between ESTn $(\mathrm{F})$ and whole embryos $(\mathrm{A})$ indicated common targets such as Celsr1, Sall1, however, the majority of responses were not concordant in directionality $(55 \%)$ between the two systems (Figure $4 \mathrm{Cb}$ ). Likewise, we observed genes which correlated directionally (up/down) in comparisons between ESTc and mouse embryo (A) with $\mathrm{MeHg}$ (e.g. Gata3, Tbx3), but also genes differentially expressed in terms of direction $(39 \%)$ (Figure 4Cc). Interestingly, in both comparisons (Figure 4Cb, c), we observed a high representation of downregulated genes in the ESC model systems which were upregulated in the whole embryo study (Quadrant III). With these broad assessments of all commonly MeHg-altered developmental genes, it is no surprising to observe imperfect correlations due to the character of the underlying model comparison. ESC systems, in theory, possess characteristic which may mimic partial representation of the whole organism developing in vivo. For example, the ESTc model represents a subtype of progenitor cell on the track of differentiating into beating cardiomyocytes. However, we may not necessarily expect to see precise correlations at the gene level in comparing 
a cell line to whole embryo due to the complexity of what is being assessed at the embryo level (multiple tissues, dilution of response, etc.). Interestingly though, comparisons between ESTc and mouse embryo (A) were greatly improved when only comparing the subset of developmental genes related to heart development (Figure 4Cd). These results suggest that specific pathway(s) or a specific process may be impacted similarly between the two models, enabling the identification of a subset of genes in which $\mathrm{MeHg}$ targets in both systems. Again, due to the limitation of these comparisons, our conclusions need further verification, but form a basis for future comparative analyses. Gene expression alterations associated with developmental signaling and hear development across ESC and in vivo-based models may serve as possible predictors to assess developmental toxicity in the ESC model system. Specific pathways (or genes) may correlate, in terms of response, in both these representative simple and complex (in vivo) systems in connection with adverse developmental outcomes.

In total, we identified 53 genes to be commonly altered (4/5 studies) across developmental models (A, B, E, F, G) (Figure 6). In general, directionality in $\mathrm{MeHg}$ response was not necessarily agreeable across all conditions across al five studies. The response associated with $\mathrm{MeHg}$ in these 53 genes indicates the promise of identifying overlapping targets across multiple in vivo and in vitro developmental models and the potential ability to derive predictive gene sets across in vitro and in vivo models, but also illustrates the true complexity due to the influence of multiple factors on response (e.g. dose, time, model, platform). Comparisons between neuroblastoma (P19) cells and mouse embryos exposed to valproic acid support the ability to identify common toxicogenomic responses across in vivo and in vitro developmental models [76]. Studies like these, which evaluate compound-induced response across in vivo and in vitro developmental models may greatly assist in determining the applicability domain of in vitro models in their ability to correlate with in vivo model hazard identification and toxicity prediction.

In this study, we did not focus on overlapping single gene changes across all eight studies due to limited common response (see supplementary data origina article, table 2). From the $\mathrm{GO}$ level, $\mathrm{MeHg}$-induced developmentally related genes were not enriched within the brain $(C)$, kidneys $(D)$ and $M E F(G)$ studies. These results do not necessarily translate into developmentally-related genes not being impacted in these systems. In fact, many developmental genes are impacted in these systems. However, the strength of signature is weaker in these designs/models in terms of the proportion of genes impacted by $\mathrm{MeHg}$ related to development as compared to other processes. Studies assessing expression profiles across models under exposure and control conditions may provide insight into differences in toxicant-induced developmental-related signatures.

Also, in this study, we did not attempt to focus on comparisons at the gene level between different platforms (Affymetrix, Codelink, Operon) due to the added complexity of platform specificity [68, 288]. Differences in $\mathrm{MeHg}$ response were apparent at the gene level even between similar studies A (Affymetrix) and B (Codelink), both assessing $\mathrm{MeHg}$ response in C57 mouse embryos undergoing neurulation (not shown). Close examination of Figure 5 reveals some of these differences in the directionality of expression in overlapping genes between mouse embryo studies (A, B). Despite these observations, strong similarities were observed between these two studies at the functional level (Figure 1A, C), supporting the use of programs which identify gene expression changes at a higher functional level (pathway, GO-level) as previously observed in comparisons between non-developmenta models [304, 305].

\section{@8}

\section{Conclusion}

Toxicogenomic studies conducted in developmentally-related models produce 1 valuable datasets which allow comparison of specific responses across in vitro and in vivo developmental models. Mechanistic-based markers (e.g. RNA, proteins, etc) may improve assessment of potential toxicity by providing an objective measure which can be associated with developmental anomalies. As with all toxicological models, response and toxicity is dependent on multiple factors such as dose and time. Here, in this study, using $\mathrm{MeHg}$ as an example developmental toxicant, we show promise of these types of toxicogenomic comparisons, but also the complexity in evaluating transcriptional responses across models. More intelligent design is needed for future studies which consider for comparable factors related to dose, time, kinetics, developmenta time, microarray platform, species and other experimental variables to make proper comparisons across developmental models.

\section{Loxistar}




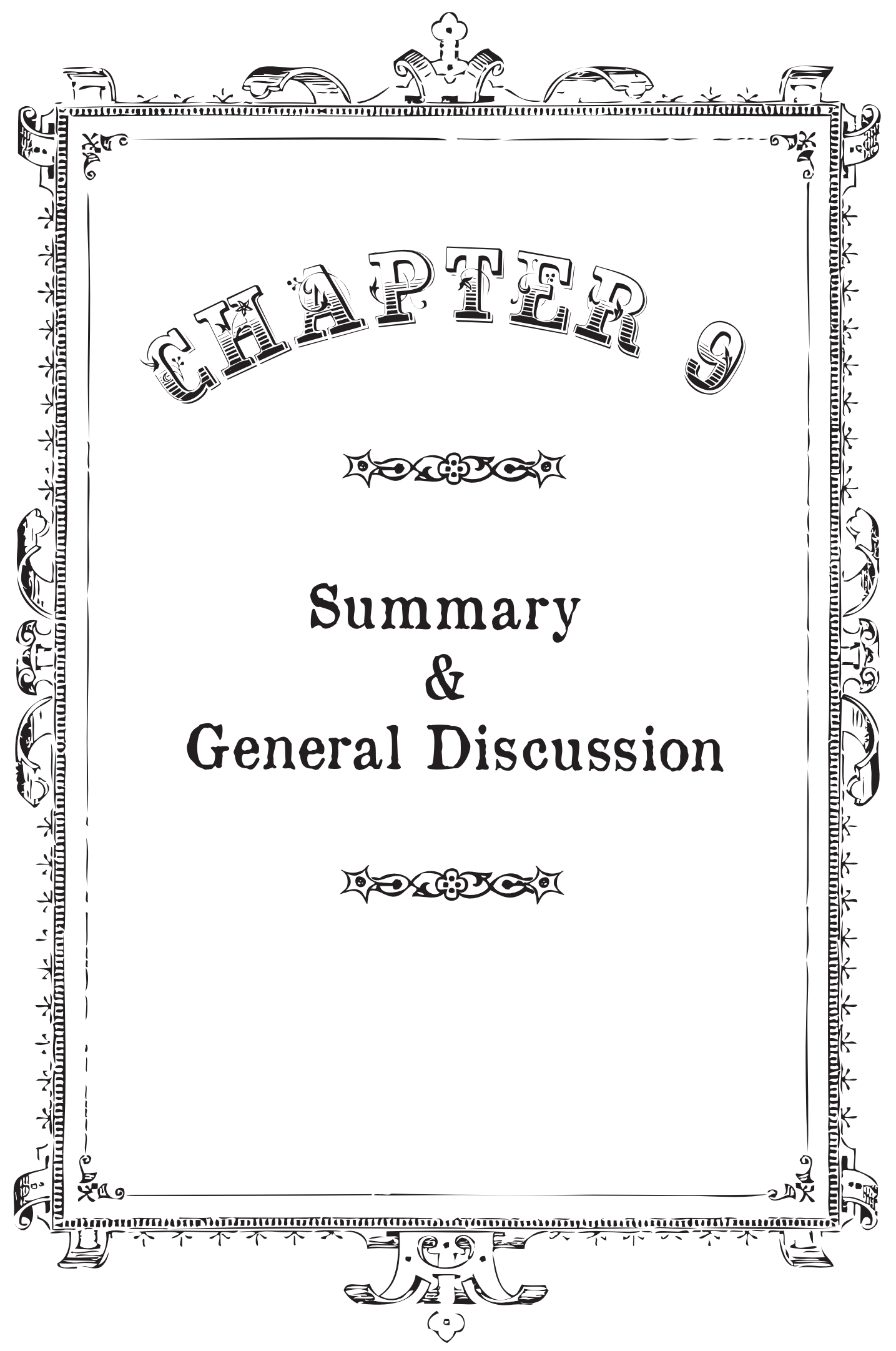




\section{Summary of main findings}

$\mathrm{N}$ this thesis the development of the neural embryonic stem cell test (ESTn) 1 as a high throughput system for detection of compound induced neurodevelopmental toxicity is described: from developing and characterizing the model to implementation of transcriptomics techniques in the testing strategy and finally the formation of a biomarker gene set for neurodevelopmental toxicity in this test system. In addition, the time-related and compound induced gene expression changes across ESTn and other in vitro and in vivo models are compared. A schematic summary of the main findings is provided in Figure 1.

Development and molecular characterization of the ESTn is described in chapter 2. A 20-day neural differentiation protocol was designed based on two existing protocols published by Okabe et al. and Bibel et al. [52, 103]. The main steps in the protocol are embryoid body (EB) formation using the hanging drop method, exposure of the EB to the morphogen retinoic acid (RA) at a physiological concentration in a suspension culture, step-wise reduction of the serum percentage from $20 \%$ to $0 \%$ in the medium and addition of an extra-cellular matrix and growth factors stimulating neural differentiation. A the end-stage of the differentiation culture, EB were for $75-100 \%$ surrounded by neural outgrowth (Figure 2).

In order to increase the high-throughput of the test system, two additional shorter variants of the protocol (13 and 17 days), with a reduced 'serum-free' phase were designed and the three protocols were characterized morphologically and at the molecular level for development related protein and mRNA markers using immunocytochemistry, gene expression and flow-cytometry. Early in the protocols, which are identical the first seven days, markers for al three primary germ layers are expressed and in addition, an increase in ectodermal and specific neuron type differentiation was observed, whereas pluripotency markers were decreased over time. This demonstrates neural differentiation was induced early in the protocols. Furthermore, at the end-stag of the three protocols, expression level of specific neuron type markers was comparable between the three protocol variants, except for GFAP, a marke for astrocytes, for which gene expression was lower but still significantly present in the shortest protocol. Due to the shared morphological and molecular characteristics of the three protocols, further experiments to test compound induced effects on neural differentiation were performed in the shortest protocol.

As a proof-of-principle, perturbation of neural differentiation by the model neurodevelopmental toxicant methylmercury $(\mathrm{MeHg})$ was assessed at multiple 
Chapter 2 .

Development and characterization of a neural differentiation protocol (ESTn). MeHg exposu
concentration dependently decreases neural outgrowth

Chapter 3

haracterization of ESTn shows time-dependent gene expression changes. MeHdg upregulates neural differentiation genes

Chapter 4 Iranscriptomics concentration-response identifies compound

Chapter 5

Mechanistically diverse compounds show similar morphological effects. Adaptive versus adverse effects are detected at process level

Chapter 6

De novo analysis generates gene set of 29 gene

predictive for neurodevelopmental toxicity
in FSTn with $84 \%$ accuracy

Chapter 7

differences at gene expressio

evel explain specific differentiation other on prediction of developmental toxcity
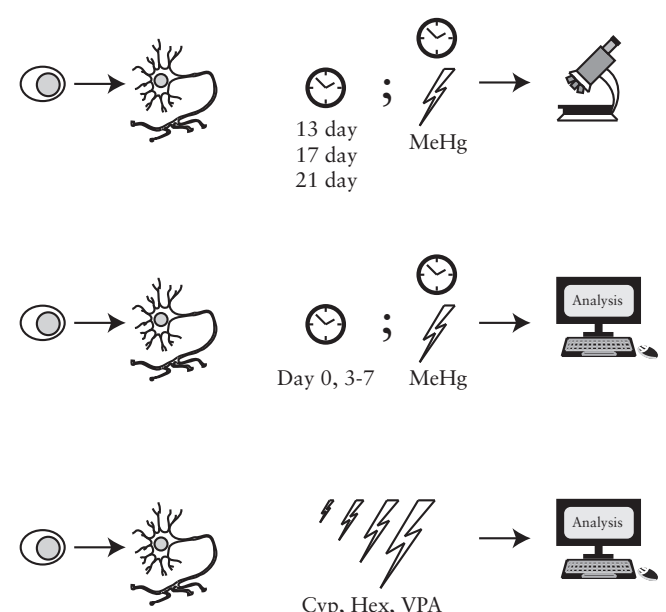

Cyp, Hex, VPA

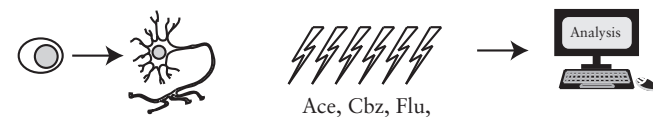

Ace, Cbz, Flu,
MFHP, PenG, Phe

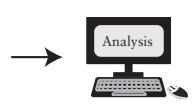

Data Chapter 3, 4,

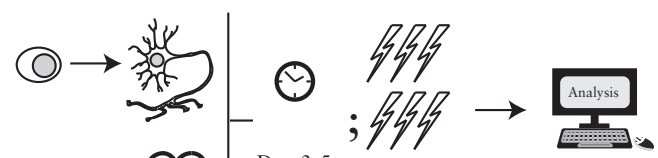

(2) $\rightarrow \bigcup \begin{array}{r}\text { Day 3-5 Cbz, Flu, Hex } \\ \text { MeHg, MEHP, PenG }\end{array}$

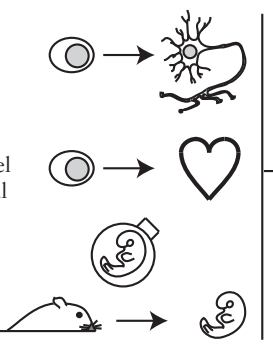

Figure 1 A Schematic summary of the main findings

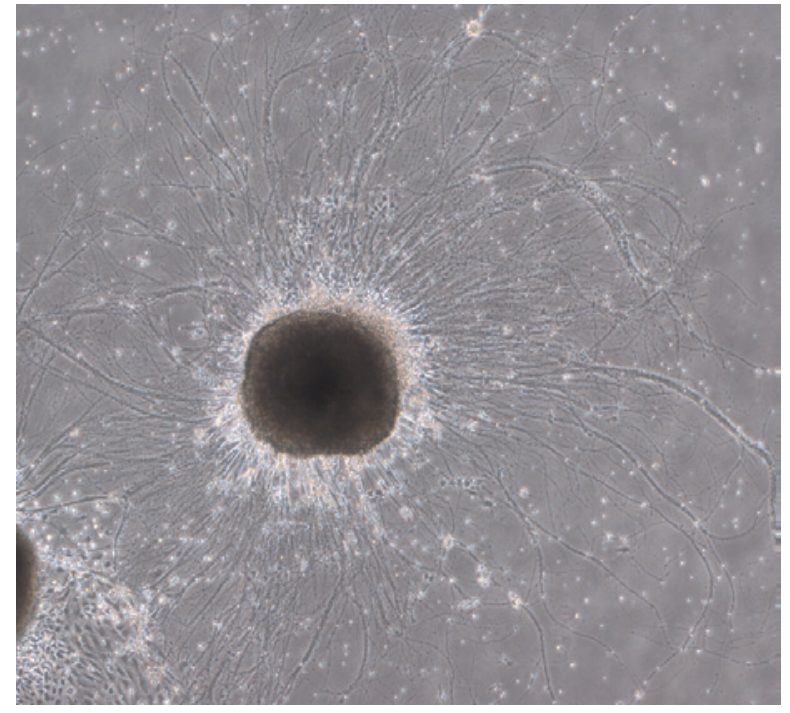

Figure 2 Example of an embryoid body (EB) completely surrounded by neural outgrowth.

concentrations. Neurite outgrowth was assessed by morphologically scoring the percentage of the EB surrounded by neural outgrowth and by measuring the amount cells positive for the pluripotency marker SSEA1, neural precursor marker nestin and neuronal marker $\beta$ III-tubulin. Due to the possible binding of $\mathrm{MeHg}$ to proteins in the serum, three different $\mathrm{MeHg}$ exposure designs were performed: exposure only during the serum containing period (days 3-5), only during the serum-free period (days 6-13) or a complete exposure (days 3-13). $\mathrm{MeHg}$ exposure effects on morphological neural outgrowth at the serum-only and complete exposures showed a similar concentration response, whereas the serum-free exposure was more sensitive. An explanation may be that at start of the serum-free exposure, the cell population in culture is different compared to the population with exposure from day 3 onwards. Furthermore, a complete absence of neural outgrowth observed exclusively after late exposure (serum-free and complete exposure) to the highest concentration tested, may be related to cytotoxicity of the compound at this concentration, which may be more pronounced under serum-free conditions. Due to the similar concentration-response observed after serum-containing and complete exposure and possible effects of lack of protein binding in the serum-free phase of the method, exposure for following experiments was performed from day 3-5. A scheme of the final version of the ESTn is depicted in chapter 2, figure 1. 
In chapter 3 the ESTn is further characterized by monitoring temporal gene expression changes related to differentiation. Cultures were sampled at days 0 , 3, 4, 5, 6 and 7 of the ESTn protocol and whole genome mRNA expression was assessed. Temporal regulated genes could be subdivided into eight separate clusters, each describing a distinct gene expression pattern over time. Multiple analysis techniques (including hierarchical clustering, DAVID and Tox-profiler) showed that early up-regulated gene clusters were involved in processes related to transcription and pluripotency and that late up-regulated gene clusters were related to processes involved in neural development. Furthermore, using principal component analysis (PCA) a differentiation track describing differentiation over time could be constructed with all genes regulated over time, as was shown before for cardiac differentiation in the cardiac stem cell test (ESTc) [187]. The differentiation track showed that for each time-point, samples clustered together, but that variation between samples increased over time, indicating cells in culture differentiated to become more heterogeneous in gene expression profile.

$\mathrm{MeHg}$ induced effects on gene expression over time were studied after 24, 48, 72 and 96 h exposure, starting from day 3 of culture onwards. $\mathrm{MeHg}$ was observed to up-regulate neural development related processes and down-regulate general development related processes and to a lesser extent, endoderm and mesoderm related processes. In addition, the PCA-based differentiation track method was used to study temporal $\mathrm{MeHg}$ effects on gene expression after 24, 48 and $72 \mathrm{~h}$ exposure. In this approach, a differentiation track is set up using the genes regulated by the time-matched control and the two control days surrounding the day of interest (e.g. for $24 \mathrm{~h}$ exposure, controls days 3, 4 and 5 are used to setup the track). Then the exposed samples are compared to its day-matched control and significant deviation from the track is calculated. $\mathrm{MeHg}$ induced samples were most significantly deviating from the differentiation track after $24 \mathrm{~h}$ exposure. Based on these results, for following transcriptomics experiments a $24 \mathrm{~h}$ compound exposure with controls on day 3,4 and 5 was performed.

Compound induced concentration-dependent effects on gene expression during embryonic stem cell (ESC) differentiation were studied in chapter 4 Three known (neuro)developmental toxic compounds were selected as tes compounds; the triazoles cyproconazole and hexaconazole and the anticonvulsant valproic acid (VPA). Compound-mediated effects on gene expression were identified after $24 \mathrm{~h}$ exposure in relation to morphologica changes on day 11 of culture. All three compounds showed a concentrationdependent effect in gene expression and biological processes, providing information on mechanisms and concentration-response characteristics.
General and specific effects on development could be discriminated between compounds. For example, concentration-dependent gene expression for the biological process embryonic morphogenesis was enriched for all compounds, indicating an effect on general development. However, cyproconazole and VPA (both neurodevelopmental toxicants) but not hexaconazole (developmenta toxicant) significantly enriched the neuron development process. In addition, compound class specific mechanisms could be detected, including sterol biosynthetic process enriched by the triazoles. Implementation of transcrip tomics in this study was shown to precede morphological changes and provided a more sensitive measure to assess possible developmental toxicity.

In order to increase knowledge on the applicability domain of the test system, effects on gene expression of six additional compounds with a differen mechanism of action was assessed at equipotent and non-toxic concentrations in chapter 5. Five compounds tested were known (neuro)developmenta toxicants (acetaldehyde, carbamazepine, flusilazole, monoethylhexyl phthalate (MEHP), and phenytoin) and penicillin G was tested as a negative control. Compounds induced transcriptomic profiles were assessed at two concentration for gene expression profiling; a toxic concentration inhibiting differentiation by approximately $50 \%$ or a concentration at which $80 \%$ of the cells was still viable and a non-toxic concentration thirty times lower. Both compound-specific and common gene expression changes were observed between subsets of tested compounds, in terms of significance, magnitude of regulation and functionality. However, overlap between the compounds was very low, indicating the specific mechanisms behind each of the compound's developmental toxicity. In addition, gene expression and GOBP enrichment showed concentration dependence, allowing discrimination of non-toxic versus toxic concentrations on the basis of transcriptomics. This information may be used to define adaptive versus toxic responses at the transcriptome level.

In chapter 6, transcriptomics data from chapters 3,4 and 5 is used to identify a biomarker gene set to predict neurodevelopmental toxicity in the ESTn. Gene expression responses were distinguished using the differentiation track algorithm with data from ten compounds and nineteen exposures. Using classification feature selection in combination with a leave-one-out cross-validation, a set of 29 genes was obtained consisting of genes mainly involved in neural development. This set allowed for $84 \%$ prediction accuracy and may contribute to further improve ESTn transcriptomics studies aimed at compound risk assessment.

The ESTn was developed as a companion test system to the ESTc, therefore differences and similarities in transcriptomics responses between the two 
systems are discussed in chapter 7. Gene expression profiles of both ESTn and ESTc over time early in both methods (days 3, 4 and 5) and changes induced by seven compounds, including six in vivo developmental toxicants and one negative control, are compared. Although temporal gene expression responses were generally comparable between models, a relatively small group of genes could be identified behaving differently in each model. These genes were mainly related to cardiac development and early developmental signaling pathways in ESTc and neural development and retinoic acid signaling in ESTn. Compound induced gene expression changes were generally different in either model, predicting mechanism behind compound developmental toxicity better in one model or in the other (e.g. MeHg in ESTn and FLU in ESTc). However, VPA induced gene expression changes were comparable in both ESTn and ESTc, but varied at the process level. A comparison between the two models shows that a tiered approach of compound screening in ESTc and ESTn may improve prediction and understanding of the compound mechanism of action. A transcriptomic based in vivo versus in vitro comparison is performed in chapter 8 in order to obtain insight on differences in prediction and mechanism of action in different models. The impact of $\mathrm{MeHg}$ on a diverse set of in vitro and in vivo models was assessed. Models predicting developmental toxicity were the in vitro rat whole embryo culture (WEC), ESTc and ESTn and two mouse-embryo developmental toxicity studies. Models predicting genera toxicity were an embryonic fibroblast system, adult rat liver brain studies. Evaluation of common and unique aspects at the functional and gene level showed stronger similarities in $\mathrm{MeHg}$ response between the development related studies compared to the general toxicity studies. This comparison shows in vitro / in vivo similarities supporting the relevance of in vitro developmental models for predicting in vivo developmental toxicity.

\section{क्ष}

\section{General discussion}

\section{Development of a neural differentiation cell culture}

ESC differentiation models are currently very promising as alternative and animal-free models for developmental toxicity [306]. Due to the pluripotent nature of ESC, in theory the effect of compounds on any differentiation route of the three germ layers can be studied, which is not possible in primary cel lines, immortalized cell lines, or cells already in a more advanced stage of differentiation, such as neural progenitor cells (NPC) [19, 130-132]. In the ESTc, the ESC default differentiation pathway is induced, mainly differentiating cells towards mesodermal and endodermal lineages, resulting in approximately $40 \%$ cardiomyocytes at end of culture. Due to the relatively weak prediction of the ESTc for neurodevelopmental toxicants such as $\mathrm{MeHg}$, the need for addition of a neural differentiation based EST was recognized [17, 36, 37].

As was described in chapter 2, we developed the ESTn as a companion model to the ESTc, leaving as many as possible characteristics of the original ESTc differentiation model intact. This approach introduced advantages, but also some minor disadvantages of the ESTn protocol compared to other possible methods. Similarly as the ESTc, standardized embryoid body (EB) formation using the hanging drop method is implemented in the ESTn, decreasing variation in EB size compared to spontaneous EB formation [21]. Three-dimensional EB formation furthermore allowed differentiation to mimic the in vivo developmental process in a more complete manner compared to monolayer differentiation, due to the presence of all three germ layers [307]. However, in the EB no physical structures such as formation of a neural tube or blood vessels is observed. The three-dimensional induced multi-lineage character of the assay may allow improved detection of developmental toxicity in the assay. On the other hand, this three-dimensional complexity can be a disadvantage when a relatively simple morphological readout for compound effects on differentiation is preferred. EB structure is maintained throughout the complete protocol, from EB formation to the morphological readout, supporting stable culture conditions. In previously described ESC neural differentiation methods for neurodevelopmental toxicity, ESC monolayer culture was used [106], or EB were dissociated to single cells during the protocol [41], ensuing a less stable protocol. Further similarities between the ESTn and ESTc, the suspension phase after the hanging drop phase and plating of the EB on day 5, are maintained to keep comparability between protocols.

In order to induce neural differentiation, three distinct steps are implemented in the protocol, which were earlier described in neural differentiation protocols by Okabe et al. and Bibel et al. [52, 103]. The first being addition of the morphogen all-trans retinoic acid (RA) to the culture at the suspension phase at a physiological concentration during neural development in vivo [308]). The first developed ESC neural differentiation models already used RA as a morphogen to induce differentiation [52]. RA, an active metabolite of vitamin $\mathrm{A}$, is known to play an important role in normal embryogenesis. Subtle gradients of RA direct morphogenesis throughout the embryo, including limb development and neural development [271-273]. In addition, during early 
organogenesis in vivo, RA levels are observed to be highest in the neural tube [272]. The second critical step in the ESTn is gradual serum deprivation of the medium as an induction and selection for neural cell types. Serum deprivation is currently widely used to induce ESC neural differentiation and was first described in an ECC cell line [56, 309]. In ECC, serum deprivation was described to induce neural differentiation and in addition induce apoptosis in other cell types which depend on serum for growth [309, 310]. However, specific mechanisms behind the effects of serum deprivation on neural differentiation remain unclear. The last critical step in the ESTn is facilitating neura growth by offering a an extracellular matrix (laminin and poly-L-ornithine) and addition of growth factors to the medium [311]. These growth factors include progesterone, sodium selenite, transferrin and bFGF and are used in serum free primary neuron cultures to create a viable environment for neura cell types [308]. Together RA supplementation, serum deprivation, the extracellular matrix and addition of growth factors create the environment for ESC to differentiate into neural cell types in ESTn.

\section{Molecular characterization of ESC neural differentiation}

\section{Characterization at the single gene level}

Characterization of differentiation over time in ESC differentiation cultures has been performed using distinguished gene expression markers for differentiation of all three germ layers, as was also performed in ESTn in chapter 2 [19]. Using this approach, differentiation can be studied in broad terms with markers for pluripotency (e.g. Pou5f1 and SSEA1), markers for progenitor cells of the three germ layers (e.g. T, nestin, Nkx2-5) and markers for mature cell types (e.g. Myh6, Tubb3, GFAP), which has shown characteristics reminiscent of germ layer development and specific lineage differentiation in ESTn and other models $[19,312]$. Although this approach can provide information on general temporal regulation of predetermined differentiation routes, obtained information remains limited due to the pre-selection of genes.

\section{Characterization at the whole genome level}

In recent years, transcriptomics analysis have provided a more complete view of ESC differentiation dynamics. A number of studies published described a range of differentiation routes, including cardiac, neural and pancreatic differentiation $[80,169,313-315]$. In these studies it was confirmed that gene expression profiles of specialized cellular differentiation protocols were concurrent with functional processes observed for specific regions of the embryo during development. However, in these studies transcriptome profiling was performed on very distinct morphological stages during ESC differentiation with relatively much time between time-points studied, by which especially during early ESC differentiation, subtle short changes in gene expression were not identified. More recently, early temporal gene expression changes over a relatively small time-span (one day or less) were studied in a number of ESC differentiation protocols, including the ESTn and ESTc, as described in chapter 3 and chapter 7 [177, 187, 235]. In these studies, it was shown that the continuous developmental changes occurring in various mouse and human ESC differentiation models could be visualized using PCA to describe a 'differentiation track' $[177,187,235,316]$. This approach makes it possible to visualize and quantify differentiation status and developmental potency status of cells cultures. In the ESTn, the differentiation track over time showed that all time-points clustered together and separate time-points were ordered chronologically. However, over time, variation between samples increased, probably due to increased heterogeneity of the cell culture. Studies on ESC differentiation have been instrumental in describing differentiation processes, showing a reduction of pluripotency and an induction of neural differentiation-related gene sets over time [177, 187, 235]. In addition, these studies showed that early ESC differentiation in diverse differentiation protocols is characterized by a cascade of time-dependent early-, mid- and late-regulated gene expression profiles and processes. For instance, in ESTn, ESTc and other ESC differentiation models, genes and processes involved in pluripotency (including Pou5f1, Nodal and Eomes) and proliferation (including Ccnd1 and Ccne1) were up-regulated early and down-regulated over time, genes up-regulated for a short duration during the midterm of the protocol related to early differentiation (T, Cyp26a1, Frzb, Lhx1) and genes and process up-regulation of (end-stage) differentiation as a late response (including Tubb3, Pax6, Gata6 among others). These 'coordinated waves' of differentiation have also been observed in vivo during the gastrula stage in multiple species, including the mouse [317].

Comparing temporal gene expression profiles in ESTn and ESTc

In order to further characterize early ESC differentiation, in chapter 7 early temporal gene expression signatures (days 3, 4 and 5) for neural differentiation in ESTn and cardiomyocyte differentiation in ESTc were compared. Thi analysis showed for a large part ( $71 \%$ of genes regulated in either model) early gene expression profiles in the two models were regulated similarly, illustrating that temporal differentiation related genes are conserved in both models. In addition, genes regulated early specifically in ESTn $(12 \%)$ could be related to connected processes involved in patterning (mainly homeobox related), RA 
metabolism and neural development, whereas genes regulated specifically in ESTc $(17 \%)$ were related highly connected to Wnt-, Tgf $\beta$ - and BMP signalling and cardiac development, showing early gene expression responses are highly related to the morphological phenotype later in the protocols.

\section{Temporal gene expression profiles in ESC differentiation in context} of in vivo organogenesis

A useful in vitro model should to a certain extent be comparable to the in vivo situation [318]. Mouse ESC are collected in vivo from the inner cell mass of the blastocyst, approximately 4 days after fertilization [19]. Therefore, the first week of ESC differentiation is expected to be correlated to day 4 - 10, during gastrulation of the developing embryo [19]. Mitiku and Baker performed a very detailed genomic profiling of gastrulation and organogenesis in the mouse embryo from day 6.5 through 9.0 post conception, which was compared to ou data in the discussion in chapter 3 [171]. Seven gene clusters were identified, describing specific processes during development, including two cluster involved in neurogenesis and cardio-vasculature development, which were up-regulated from day 8.0 onwards, in a comparable manner to late responses observed in ESTn and ESTc [171, 177, 187]. Interestingly, another cluster involved in blood vessel development and neurogenesis consisted of genes with a peak up-regulation at day 8 , which was again down-regulated at day 9.0, similar to the observation in ESTn and ESTc. Comparisons of gene clusters with late expression profiles in ESTn with the in vivo data were performed and corresponding genes, and GO terms were observed. Although evidence is limited, this similar gene signature provides an additional indication that processes involved in ESTn and ESTc are comparable to the in vivo situation.

\section{ESTn as a test system for assessment of neurodevelopmental toxicity}

Developing an endpoint for neurodevelopmental toxicity in ESTn

For detection and prediction of adverse effects on neural differentiation in the ESTn, a readout method was needed. In earlier described models for neurotoxicity and neurodevelopmental toxicity, a number of readouts have been proposed [319], including cell morphology by assessing neural outgrowth, axonal and dendritic morphology, cytotoxicity/viability and migration in monolayer or neurosphere cultures [149, 150], functional parameter determined by electric potential or calcium homeostasis $[147,320]$ or assessing neural differentiation using mRNA transcripts of a small selected number of genes $[41,321]$. In order to perform quick readout screens, a relatively fast readout system was required for ESTn. Due to the complexity of the neura outgrowth at the end of culture, fast measurement of functional parameters was not possible. Therefore, initially a combination of a morphological and molecular approach was chosen, assessing neural outgrowth from EB microscopically and compound effects on protein markers using flow cytometry, as was described in chapter 2. Morphology of neural outgrowth was scored a the percentage of neural corona surrounding each $\mathrm{EB}$, irrespective of the distance of outgrowth from the EB. A scoring was performed for 100, 75, 50, $25,<10$ and $0 \%$ neural outgrowth surrounding the EB (Figure 3). For scoring compound effects on neurite outgrowth, a cutoff was chosen at $\geq 75 \%$ neura outgrowth around the EB, based on historical control data. Thus, when more than $75 \%$ of the corona around an EB contained microscopically observable neural outgrowth, the EB was scored positive, whereas below $75 \%$ it was scored negative. Using a concentration-response curve, the concentration by which a $50 \%$ inhibition of differentiation (ID50) (EB with $>75 \%$ neural outgrowth) was observed, could be identified. This ID50 method has also been used to define developmental toxicity in the EST [17].

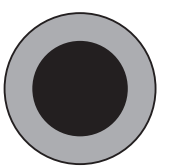

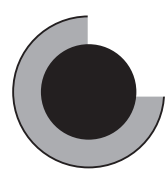

$75 \%$

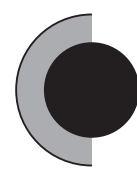

$50 \%$
Positive

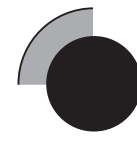

$25 \%$

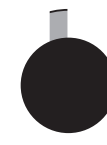

$<10 \%$
Negative

Figure 3 Schematic overview of Morphological Scoring criteria for determining the percentage of neural outgrowth around the EB.

Next to morphology, initially flow cytometry was used to count the number of cells positive for the pluripotency marker SSEA1, neural precursor marke nestin and neuron marker $\beta$ III-tubulin. Flow cytometry has been successfully implemented in the validated EST using the cardiomyocyte marker MYH as readout parameter [322]. As a proof-of-principle, effects of the model neurodevelopmental toxicant $\mathrm{MeHg}$ were assessed using the morphology and flowcytometry readouts. A strong concentration-response was observed in the morphological readout, but only limited prediction was observed by the flow cytometry method. Therefore, for further experiments, morphological scoring 
of neurite outgrowth was used as a parameter to define compound effects on neural differentiation and outgrowth.

\section{Exposure strategy: Selection of exposure duration and} concentration ranges

Due to the complexity of the ESTn, including changes of serum percentage in culture medium and the differentiating character of the culture cell types, effects of compound exposure duration and concentration ranges should be taken into consideration, in order to obtain an optimum exposure strategy for assessing compound perturbation on neural differentiation in ESTn (chapte 2). The ESTn was designed to detect compound induced effects specifically on neural development. In an optimal setting, a compound has an effect on neura differentiation, but not on cell viability or cytotoxicity [17]. Therefore, a viability assay (the Resazurin assay) on ESC was performed to determine the cytotoxic effects of a compound, and to determine a concentration-range to be tested in ESTn [323]. Furthermore, previous studies performed in ou laboratory showed that proliferation and differentiation of ESC in the ESTc are highly intertwined processes [137]. It was recommended that, to largely limit exposure to the proliferation phase of the assay, it would be advantageous that EB be exposed to compounds from day 3 in the assay onwards [137]. Up to day 3, the ESTc and ESTn are identical. Therefore, compound exposure was performed from day 3 onward in all studies. Another variable influence on exposure in culture was serum deprivation of the culture medium [324]. Effects of $\mathrm{MeHg}$ exposure only during the serum-containing period (days 3-5), only during the serum-free period (days 6-13) or a complete exposure (days 3-13) was assessed. Due to the similar concentration-response observed after serumcontaining and complete exposure and possible differences in exposure due to compound binding to protein between the serum-containing and serum-free phase of the method, exposure for following experiments was performed from day 3-5. In addition, temporal transcriptomics profiling of ESTn differentiation described in chapter 3 showed that greatest changes in gene expression are observed early in the ESTn (day 3-5) compared to later (days 6 and 7), indicating greatest compound effects can be expected during this stage of culture [177].

\section{Limitations of scoring of neural outgrowth as a readout for neuro-}

\section{developmental toxicity}

Although the morphological readout in ESTn was successful in predicting neurodevelopmental toxicity for a number of compounds, including $\mathrm{MeHg}$, valproic acid, carbamazepine and phenytoin (chapters 3,4 and 5), there are limitations to this readout. The scoring only takes into account the morphological outgrowth, however, this method does not take into account effects on the variety of cell types in culture, such as shifts in ectodermal versus other germ layer type cells or even differences in neuron types or neuron versus astrocytes [319]. In addition, as stated before, neuron functionality cannot be taken into account using this model. Furthermore, morphologica scoring provides insight on the ultimate effect of a compound on neurite outgrowth, while the adverse compound effect might have occurred much earlier in the differentiation process, as was observed in ESTc [155]. Although the morphological readout in ESTn and other readouts in previous test systems show promise in being used for the prediction of neurodevelopmental toxicity, the complexity of the developing nervous system warrants a more comprehensive assessment at the molecular level of exposure effects by neurodevelopmenta toxicants [60].

\section{Implementation of transcriptomic techniques in ESTn for} prediction of neurodevelopmental toxicity

The usefulness of transcriptomics as a mechanistic approach for prediction of (neuro)developmental toxicity has been shown in vivo and in vitro [79, 80, 82, 151]. The use of molecular screens for toxicity testing is furthermore predicted to play an important role in near-future risk assessment [217]. The aim of transcriptomics implementation in the ESTn was to increase test throughput by measuring compound toxicity at an earlier stage, gain additional mechanistic insight in compound specific toxicity and to find biomarkers for (neuro) developmental toxicity $[177,263]$. Performing a time-response transcriptomics study to determine most robust gene expression changes over time has been proven useful before in mouse developmental toxicity studies [152]. In order to determine the most informative exposure duration, $\mathrm{MeHg}$ exposure effects at the morphological ID50 concentration after 24, 48, 72 and 96h exposure were assessed in chapter 3. Using multiple analysis tools, from the single gene leve to process enrichment, $\mathrm{MeHg}$ was observed to increase neurectodermal related genes and decrease mesodermal, endodermal and early differentiation genes over all time-points. Similar observations made in the WEC and in vivo in mice confirmed that transcriptomics in the ESTn can provide additiona information on compound mechanism of action $[82,152]$. In vivo mechanism of action was later also verified in ESTn for multiple other compounds in chapter 4 and chapter 5, including VPA, cyproconazole and flusilazole [203, 263].

In order to make a statistical prediction for compound induced neurodevelopmental toxicity in ESTn, the differentiation track approach was used in chapte 3 determining best exposure timing, chapter 5 to explain differences in 
mechanism of action between compounds and for biomarker discovery in chapter 6 . The differentiation track approach was first described by van Dartel et al. [187]. Using this technique, differentiation over time can be visualized by PCA and compound induced deviation from the track can be statistically defined [187]. Earlier we observed an increase in variation of late time-point samples for differentiation over time, indicating an increased heterogeneity between samples of one time-point [177]. Furthermore, greatest dynamics in gene expression changes were observed at day 4 (which is the same time-point as a $24 \mathrm{~h}$ exposure) on the differentiation track compared to the remaining time-points, as was also observed in ESTc [177, 187]. Compound induced effects on the differentiation track were further studied on a 24,48 and 72 hour exposure differentiation track. The 24 hour MeHg exposure provided the most significant deviation from the differentiation track, whereas 48 and 72 hour exposure samples did not deviate significantly from the track, due to increased time- and compound-induced sample variance. Furthermore, a shorter exposure of 6 and 12 hours with monobutyl phthalate in the ESTc did not induce any significant gene expression changes [40]. Therefore, based on the least variance, greatest observed dynamics in temporal gene expression and most significant compound deviation from the track, a 24 hour exposure was chosen for following experiments. Use of the differentiation track in chapter 5 was furthermore instrumental to visualize compound induced changes on differentiation [263].

In chapter 6 , the differentiation track was further used to obtain a biomarker set for prediction of neurodevelopmental toxicity in the ESTn. Using a de novo analysis, including a leave-one-exposure-out approach, a gene set consisting of 29 genes was developed using data from chapters 3,4 and 5. This gene set described both ESC differentiation and predicted neurodevelopmental toxicity. Evaluating the performance of this gene set in combination with the differentiation track algorithm showed that developmental toxicity of 10 compound and 19 toxic and non-toxic exposures could be correctly predicted with an accuracy of $84 \%$. These genes were mainly involved in processes related to neural development and general development. A similar approach was successfully performed for the ESTc for 15 compounds, resulting in a set of 52 genes with an accuracy of $83 \%$ [84]. In contrast to the prediction accuracy of $78 \%$ under the original ECVAM validated EST, the results for both ESTn and ESTc are promising [17]. However, additional compounds have to be evaluated, including negative controls and pharmacological active compounds which are not developmental toxic, to further confirm prediction of these differentiation track-based novel identified gene sets.
Implementation of transcriptomic concentration-response in ESTn

\section{to identify adaptive and adverse responses}

The value of taking into account concentration-response in combination with transcriptomics to identify compound toxic properties has been shown in various fields of toxicological research [151, 229, 230, 305, 325]. In the field of reproductive toxicology, Naciff and Daston performed a range of studies in the male and female reproductive tract, showing individual endocrine disruptor induced gene expression changes followed a dose-response relationship [73, 325-327]. Comparable findings were observed in developmental toxicity studies by Robinson et al., where exposure to the metals $\mathrm{MeHg}$, cadmium and arsenic showed a dose-dependent effect on development related gene expression and process enrichment during gestational development in the mouse [152, 279]. Furthermore, a study in the ESTc described flusilazole concentrationdependent mechanisms of action at the transcriptome level [230]. Effects on processes related to development were observed at lower concentrations than those related to sterol metabolism, followed by enrichment of cell cycle gene expression at even higher cytotoxic concentrations. Similar findings were observed in chapter 4 where exposure to VPA, cyproconazole and hexaconazole in the ESTn showed a concentration-dependent effect on individual gene expression, fold change and process enrichment. Using a concentration-response approach, mechanistic effects on development related processes could be identified at concentrations below observed effects on morphology, providing a more sensitive measure of concentration-dependent effects. Furthermore, compound specific process enrichment could be identified, enabling discrimination between the neurodevelopmental- (e.g. VPA and cyproconazole) and more general developmental toxicants (e.g. hexaconazole). In addition, compound class specific responses on sterol metabolism could be identified, discerning the triazoles from VPA.

Although using transcriptomics enables to detect compound induced effects on gene expression with greater sensitivity, a definition is required to discriminate adaptive from adverse gene expression changes. This definition should identify the level of regulation of a functional process representing an adverse response, in order to discern compound adverse effects exclusively with gene expression. This distinction has gained importance in view of interpreting gene-expression findings for risk assessment [226]. Until adverse and adaptive responses are identified, application of toxicogenomics for risk assessment remains dependen on traditional toxicology parameters [328]. Based on available human data, it has been proposed that reproductive and developmental toxicants have threshold of adversity [329]. Current in vivo developmental toxicity testing paradigms are also build upon this hypothesis $[10,11]$. In order to discern 
adverse from adaptive effects on gene expression, Daston and Naciff proposed an approach for validation of in vitro alternatives, in which not the compound per se but an "exposure", defined as a compound concentration or dose, is classified as a developmental toxicant, enabling the use of a compound exposure as both a 'positive' and a 'negative' toxicant [101]. In line with this approach ESTn gene expression responses between a non-developmental toxic and a developmental toxic exposure concentration were compared in chapte 5. The study design was aimed at distinguishing between adaptive and adverse responses at the gene expression level. The developmental toxicant carbamazepine and flusilazole were observed to enriched pathways involved in transcription and compound metabolism at the low concentrations, whereas development related processes were enriched only at the high concentration. Additionally, the low concentration carbamazepine enriched histone methylation related processes which represent a known pharmacological mechanism of action of carbamazepine [227]. Taken together current evidence in literature suggests that up to a certain level of induction, general physiologica pathways such as related to transcription and metabolism may indicate adaptive responses, whereas the induction of developmental pathways may be more specifically indicating adverse responses [151, 203, 229, 230, 305, 325] In addition, the magnitude of induction should be considered. More detailed studies and development of additional concentration-response modeling tools will be needed to define the thresholds between adaptive and adverse responses in in vitro assays, in the interest of their interpretation and in view of hazard and risk assessment [217].

\section{ESTn in risk assessment}

Risk assessment determines the risks of human exposure to a compound or pharmaceutical [330]. Regulatory risk assessment is currently based on anima toxicity studies [9, 11]. Intelligent testing strategies are used to assess toxicological profiles, using decision schemes to determine the requirements of animal studies needed for hazard assessment [331]. Intelligent testing strategies use a stepwise approach (battery or tiered) with tests of increasing complexity to gather information on compound toxicity, weighing the information to determine further testing after each test performed, with the aim of limiting the number of animals needed. To further reduce the number of animals and decrease the time consuming process of toxicity testing, enhancing the use of in vitro developmental toxicity testing methods within intelligent testing strategies is desirable. In an intelligent testing strategy, such as the OECD conceptual framework [11], initial prioritization based upon all existing nformation is followed by non-testing information such as read-across and Quantitative) Structure-Activity Relationships ((Q)SAR). Simple in vitro screening assays, such as receptor binding assays, will provide more mechanistic data and as a next step, more complex (multi)cellular in vitro models such as the ESTn, can be studied. In this phase, multiple test systems each describing part of development could be combined to provide a more complete indication of possible untoward effects on embryogenesis, functioning as a prioritizing tool for determining whether in vivo developmental toxicity studies are necessary. An initiative combining multiple in vitro models in an effort to determine compound induced toxicity in humans is the US Environmental Protection Agency (EPA) ToxCast program [332]. In this project 650 in vitro assays, including a variant on the ESTc, are combined to obtain a chemical fingerprint of currently more than 2000 chemicals. Using computational technology, it is thought a prediction model can be constructed for developmental toxicity based on high- throughput systems [266]. The optimal place of ESTn within an intelligent testing strategy is dependent on a clear definition of its applicability domain, in terms of the biological processe represented in the assay. The term applicability domain was defined by European Centre of Validation of Alternative Methods (ECVAM) as: Definition of the chemical classes and/or ranges of test method endpoints for which the model makes reliable predictions [35]. According to this definition, the predictive value of the method for different compound classes should assist in determining its applicability domain. Furthermore, the biological mechanism incorporated within the system need to be characterized, to define the scope and limitations of the assay.

\section{Defining the applicability domain}

Developmental toxicants are structurally diverse and induce a broad range of developmental defects [333]. Furthermore, it has been shown that compound inducing comparable birth defects, can do this through multiple mechanisms of action. For instance, in the WEC it was observed that four compounds inducing spina bifida in vivo showed limited overlap in toxicogenomic responses [82]. In chapter 5 a similar finding is observed in the ESTn, where five compounds inducing neural tube defects in vivo showed no overlap in gene expression responses. To deal with this issue, a compound category approach may be useful. This strategy allows the assessment of specific chemical classes and their biological mechanisms for which the ESTn can make accurat prediction. A category approach could lead to a substantial reduction in anima use for developmental toxicity testing. When a chemical class has been correctly 
predicted in vitro, chemicals belonging to the same class but with unknown embryotoxic properties could reliably be tested in the ESTn. In addition, using a read-across approach a prediction can be made as to the embryotoxic potency of that compound. Implementing a compound category approach and relativepotency ranking within a compound class have proven to be reliable methods for determining relative toxic properties in various models for developmenta toxicity [15, 78, 81, 277, 334-336]. In the ESTc, de Jong et al. were able to rank relative potency of a series of homologous compounds, glycol ether alkoxy acid metabolites, valproic acid analogs and triazoles, matching the potency ranking to in vivo ranking [15, 334, 335]. In addition, using transcriptomics, categorization of phthalates and triazoles was performed in the ESTc, using gene sets of class specific differentiation modulation [81, 337]. Furthermore, within classes, known in vivo developmental toxicants and non-developmenta toxic compounds could be distinguished using gene expression profiling [81]. In line with these studies, in chapter 4 a transcriptomic comparison between gene expression profiles induced by two triazoles, the neurodevelopmenta toxicant cyproconazole and developmental toxicant hexaconazole, was performed in ESTn. For both compounds, effects on general embryonic development terms could be identified, however, only for cyproconazole effects on neuron development were observed. Although further research is needed to determine the applicability domain of the ESTn, these results provide promising insight. To further increase knowledge on the applicability domain, a proposed method is identification of perturbations in pathways of toxicity in a model [217]. In the National Research Council's proposed $21^{\text {st }}$ century toxicity testing strategy, these pathways of toxicity are identified as the biological signaling pathways important in compound perturbation [217]. In their vision, a systems approach can be used to describe the fundamental biologic events involved in toxicity pathways and to provide evolving biologic modeling tools that describe cellular circuits and their perturbations by compounds [338]. When the specific pathways of toxicity are identified for the ESTn, the applicability domain of the test system will be better understood.

\section{Extrapolating transcriptional profiling in the ESTn to other} test systems and species

\section{Transcriptomic ESTn and ESTc comparisons}

The ESTn was developed as a companion test system to the ESTc, and in both models transcriptomics studies have been performed to determine developmental toxicity. In chapter 7 differences and similarities in transcriptomics responses between the two systems are discussed. Gene expression profiles of both ESTn and ESTc over time early on days 3, 4 and 5 are compared. Although temporal gene expression responses were generally comparable between models, a relatively small group of genes could be identified behaving differently in each model. These genes were mainly related to cardiac development in ESTc and neural development in ESTn. Gene expression changes induced by seven compounds, including six in vivo developmenta toxicants and one negative control were assessed further. Compound induced gene expression changes were generally different in either model, predicting better in one model or in the other. For instance, the neurodevelopmental toxicant $\mathrm{MeHg}$, which was misclassified in the validated EST [17], specifically induced gene expression changes related to neural development in ESTn, an only general development in ESTc. Furthermore, the tested concentration in ESTn was 200 fold lower compared to ESTc, indicating a higher sensitivity of neural differentiating cells to $\mathrm{MeHg}$. On the other hand, specific effects of flusilazole on cardiac differentiation were observed in ESTc, whereas at a comparable concentration, no specific effects on neural differentiation were observed in ESTn. Furthermore, VPA induced gene expression changes were comparable in both ESTn and ESTc, but varied at the process level. Testing of developmental toxicants in both ESTc and ESTn in a tiered approach can provide additional information for in vitro developmental toxicity prediction screens, especially when a compound is suspected to be a neurodevelopmental toxicant, as shown with $\mathrm{MeHg}$.

\section{Interspecies- and in vitro / in vivo extrapolations using}

\section{transcriptomics}

Transcriptomics assessments provide a sensitive, robust and common endpoint that can be compared across in vivo and in vitro models, which usually use a variety of model-specific morphological endpoints to determine developmental toxicity. Additionally, cross-comparisons across in vitro and in vivo models may facilitate the identification of relevant biomarkers associated with compound exposure and developmental toxicity, and increase our understanding in determining how systems correlate in terms of response to compounds.

For extrapolation between species in vivo, there is recent transcriptomics evidence that the phylotypic stage plays a critical role in development [339] Irie and Kuratani performed a quantitative comparative transcriptome analysis of four model vertebrate embryo models (mouse, chick, zebrafish and frog) and showed that the phylotypic stage is the most conserved phase in development across vertebrates (e.g. GD8.5 in mouse and 24hpf in zebrafish) [339]. During this stage, forming of the blastula, gastrulation and neurulation occur. In addition, at earlier (cleavage and morula) and later stages (somitogenesis and 
organogenesis) transcriptomic profiles between vertebrates is less conserved. Extrapolation of compound induced developmental toxicity between species at the phylotypic stage may be more relevant than at earlier or later stages. Current transcriptomic tests performed in vivo, and in the WEC, zebrafish, ESTn and ESTc are performed during this stage, increasing the probability of finding effects which can be extrapolated to the human situation [77, 82, 84 , 177, 215].

Although limited, initial comparative studies across models have shown the ability to match gene alterations linked with developmental toxicity in vivo with biomarkers in vitro. In a study by Kultima et al. [340], targets of valproic acid associated with effects on neural tube development in vivo were identified to be commonly altered in P19 neuroblastoma cells due to valproic acid. Genes commonly altered between the two models represented known neural tube defect candidate genes and pathways critical for early embryogenesis (e.g. retinoic acid). In a parallel transcriptomic comparison study between unexposed cultured whole embryos (WEC) and embryos in vivo, high similarities in gene expression changes over time were identified between the two model systems, including significance of gene expression changes, direction in regulation (increase or decrease) and corresponding functionality [341] suggesting the WEC to be a good representation of this particular window of in vivo development at the molecular level. Similarly, in response to all-transretinoic acid exposure, high similarity in gene expression changes were observed between WEC and embryos in vivo despite both common and unique developmental toxicity endpoints [77].

In chapter 8, a larger comparison study between in vivo model systems and in vitro systems, including the ESTn and ESTc, MeHg-induced transcriptomic response was compared to identify commonalities and dissimilarities in response [342]. Greater similarity were observed in terms of response between mouse embryos exposed in utero (2 studies), differentiating ESC in the ESTc and ESTn and WEC as compared to adult mouse liver, juvenile mouse brain and mouse embryonic fibroblast $\mathrm{MeHg}$-studies. $\mathrm{MeHg}$ was observed to impact developmentally-related signatures in the early development model systems (in vivo or in vitro) versus the non-traditional developmental model systems. Future comparisons between ESTn and in vivo (as well as other alternative systems) using the emerging rich toxicogenomic databases should increase the mechanistic understanding of the relevancy of compound induced response in the ESTn, and therefore, the applicability domain of the ESTn for developmenta toxicity testing.

\section{కి}

\section{Conclusion}

In this thesis the development of the ESTn and implementation of transcriptomics into the ESTn testing strategy have been described, ultimately resulting in a biomarker gene set for prediction of neurodevelopmental toxicity. Compound effects on gene expression have been studied both over time and concentrations, comparing mechanism of action of a range of neurodevelopmental toxicants. Furthermore, comparisons between ESTn, ESTc and other developmental toxicity models show the ESTn can add mechanistic insight and increased prediction to the developmental toxicity testing strategy. In order increase the prediction of the ESTn, the applicability domain of the ESTn should be further defined by defining the pathways of toxicity important in ESTn neural differentiation and compound perturbation.

\section{Toxesers}




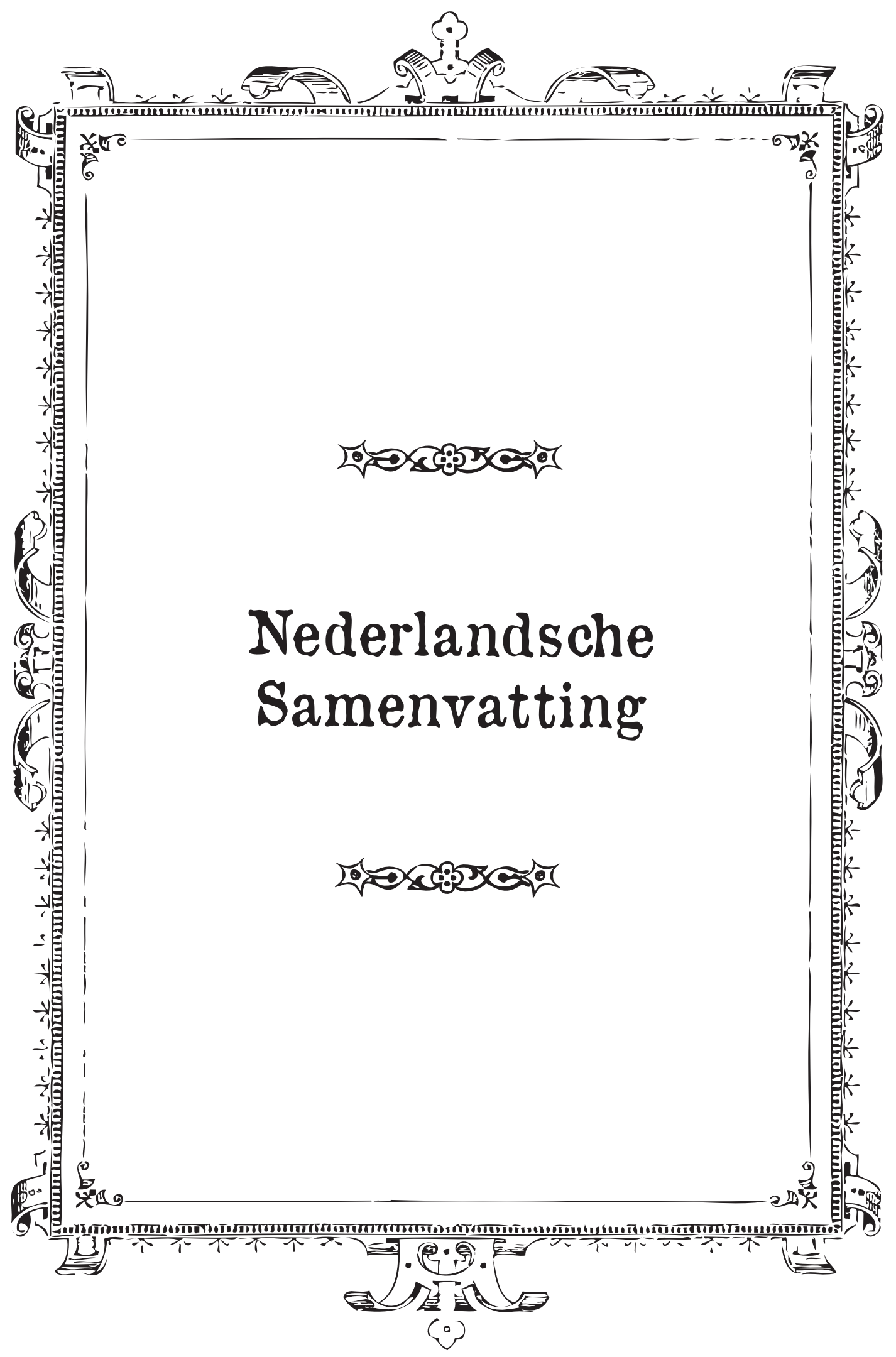




\section{Inleiding}

E wetenschap van de ontwikkelingstoxicogie houdt zich bezig met het onderzoeken van de giftige effecten van stoffen op de ontwikkeling van de ongeboren vrucht. Om geboorteafwijkingen door giftigheid van stoffen te voorkomen, verplicht huidige internationale wetgeving fabrikanten om stoffen en medicijnen te testen op ontwikkelingstoxiciteit. De bepaling van toxiciteit van een stof wordt uitgevoerd op proefdieren. Van alle proefdieren die tegenwoordis gebruikt worden voor toxicologische testen, wordt ongeveer $65 \%$ gebruikt voor ertiliteit- en ontwikkelingstoxicologie. Dit komt doordat in deze studies het effect van stoffen over meerdere generaties dieren wordt bestudeerd. Omdat het gebruik van proefdieren ethisch ongewenst is, is het belangrijk om nieuwe alternatieve testsystemen te ontwikkelen voor het voorspellen van ontwikkeingstoxiciteit, waarbij minder of zelfs géén proefdieren gebruikt hoeven te worden. Daarnaast zijn de huidige testen te arbeidsintensief om voorspellingen te kunnen doen over de giftigheid van een groot aantal stoffen in een korte tijd. Een veelbelovend alternatief model voor het voorspellen van ontwikkelingstoxiciteit is de embryonale stamcel test (EST). Deze test maakt gebruik van de pluripotente eigenschap van embryonale stamcellen (ESC), waardoor deze cellen kunnen differentiëren tot elk celtype in het lichaam. In deze test worden stamcellen gedifferentieerd tot hartspiercellen (cariomyocyten), welke onder de microscoop waarneembaar zijn doordat deze cellen contraheren. Het verstoren van deze differentiatie ten gevolge van blootstelling aan ontwikkelingstoxische stoffen wordt gezien als een maat voor ontwikkelingstoxiciteit. Dit wordt gemeten door met behulp van de microscoop 'handmatig' het aantal celculturen met kloppende gebieden te tellen. Uit validatie van de EST bleek dat de test een redelijk goede voorspelling gaf voor ontwikkelingstoxiciteit, maar niet voor stoffen welke een nadelig effect hebben op de ontwikkeling van het zenuwstelsel. Om de voorspelbaarheid van de EST te verbeteren, werden daarom een aantal voorstellen gedaan. Een van deze verbeteringen was het ontwikkelen van een neurale variant van de EST, om de giftigheid van neuraal ontwikkelingstoxische stoffen toch goed te kunnen voorspellen. Een ander voorstel was om de subjectieve handmatige scoringsmethode van de EST te vervangen door een objectievere maat. Een methode om dit te doen is met behulp van transcriptomics, een relatief nieuwe techniek waarmee de expressie van tienduizenden genen in één experiment gemeten kan worden. Wanneer met deze techniek het verschil in genexpressie tussen blootgestelde en niet blootgestelde cellen gemeten wordt, kan een profiel ontwikkeld worden welke stof geïnduceerde effecten op ontwikkeling in een testsysteem beschrijft. Daarnaast kan deze methode opheldering geven over de vele moleculaire mechanismen die ten grondslag liggen aan ontwikkelingstoxiciteit. 


\section{Samenvatting}

$\mathrm{N}$ dit proefschrift wordt de ontwikkeling van de neurale embryonale stam1 cel test (ESTn) beschreven. De ESTn is ontwikkeld als een snel, of 'highthroughput', test systeem voor het detecteren van neuraal-ontwikkelings toxische stoffen. Alle stappen, van ontwikkeling en het karakteriseren van het model, naar implementatie van transcriptomics technieken in het test systeem tot het ontwikkelen van een biomarker genen set voor neuraal-ontwikkelings toxiciteit worden besproken in dit proefschrift. Daarnaast worden de genexpressie profielen, welke de differentiatie over de tijd en effecten van stoffen beschrijven in de ESTn, vergeleken genexpressie profielen in andere in vitro en in vivo modellen.

De ontwikkeling en het karakteriseren van de ESTn is beschreven in hoofdstuk 2 Een 20 dagen lang neuraal differentiatie protocol was ontworpen, gebaseerd op twee eerder beschreven protocollen door Okabe et al. en Bibel et al. De belangrijkste stappen om stamcellen te laten differentiëren tot neurale cellen in deze methode zijn de volgende: Ten eerste de vorming van embryoid bodies (EB) door middel van de 'hanging drop' methode. Een EB is een agglomeratie van cellen die de karaktereigenschappen heeft van een zeer vroeg stadium embryo. Ten tweede worden deze EB bloot gesteld aan retinolzuur (RA), een actieve metaboliet van vitamine A. Deze metaboliet heeft tijdens de ontwikkeling onder andere een belangrijke functie in de vroege vorming van het zenuwstelsel. Ten derde wordt het serum percentage in het medium in twee stappen verlaagd van $20 \%$ naar $0 \%$ en worden groeifactoren toegevoegd aan het medium, beiden om ESC differentiatie richting neurale cellen te stimuleren. Aan het einde van de kweek worden de EB compleet omringd door neurale uitgroei, die bestaat uit neuronen en glia cellen (Hoofdstuk 9, Figuur 2).

Omdat dit protocol voor neurale differentiatie redelijk lang is voor een snelle screening van stoffen, ontwikkelden we twee kortere varianten, een 13 dagen en een 17 dagen lange methode. Dit deden we door de fase waarin het medium geen serum bevat te verkorten. De drie protocollen werden vergeleken wat betreft morfologie van de neurale uitgroei en op moleculair niveau, waarbij we keken naar RNA en eiwit markers welke belangrijk zijn tijdens de vroege ontwikkeling. Dit gebeurde met behulp van immunocytochemie, genexpressie bepalingen en flow-cytometrie. Tijdens de eerste zeven dagen zijn de drie protocollen identiek aan elkaar. Tijdens deze fase zagen we dat markers voor de drie primaire kiemlagen (ectoderm, mesoderm en endoderm) aanwezig waren in de kweek. Daarnaast werd gezien dat neurale en ectodermale markers over de tijd verhoogd waren en markers voor pluripotentie over de tijd verlaagd werden. Hiermee werd aangetoond dat neurale differentiatie al vanaf vroeg in het protocol geïnduceerd werd. Vergelijking van de eindstadia van de drie protocollen laat zien dat de mate van genexpressie voor verschillende neuronen typen vergelijkbaar is. Uit dezelfde analyse blijkt dat het genexpressie niveau voor een marker voor astrocyten lager, maar nog steeds significant verhoogd is in het korte protocol ten opzichte van de andere twee langere protocollen. Aangezien de drie protocollen wat betreft morfologie en moleculaire expressie vergelijkbaar zijn, werd voor de volgende experimenten gebruik gemaakt van het kortste protocol, wat we vanaf nu de ESTn zullen noemen.

Om te bepalen of het mogelijk was om specifieke neurale ontwikkelingstoxiciteit te kunnen voorspellen in de ESTn, stelden we de cellen bloot aan methylkwik $(\mathrm{MeHg})$ op verschillende concentraties. We bepaalden het effect van $\mathrm{MeHg}$ op de neurale differentiatie door bij verschillende EB de mate van neurale uitgroei rond de EB te bepalen. Daarnaast gebruikten we de flow-cytometrie methode om het percentage cellen te tellen dat positief was voor de volgende drie markers: SSEA1, een marker voor pluripotentie; nestin, een marker voor neurale voorlopercellen; en $\beta$ III-tubuline, een marker voor neuronen. Doorda $\mathrm{MeHg}$ aan bestanddelen van het serum in het medium kan binden, kan de effectieve concentratie en daarmee de blootstelling aan de cellen dalen. Aangezien in de ESTn het serum percentage in het medium verlaagd wordt van $20 \%$ naar $0 \%$, voerden we drie verschillende blootstellingen uit: blootstelling tijdens de serum periode (dag 3-5), tijdens de serum vrije periode (dag 6-13) of tijdens de gehele periode (dag 3-13). Effecten van $\mathrm{MeHg}$ op de neurale uitgroei waren vergelijkbaar voor blootstellingen tijdens de serum periode en gehele periode, terwijl de serum vrije periode gevoeliger was. Daarnaast was bij de hoogst geteste concentratie nog steeds neurale uitroei waargenomen bij de serum periode blootstelling, terwijl bij de twee andere blootstellingen geen neurale uitgroei meer was. Dit komt waarschijnlijk doordat tijdens de serum vrije periode meer $\mathrm{MeHg}$ vrij beschikbaar is in het medium waardoor de effectieve concentratie toeneemt. Door de vergelijkbare concentratierespons tijdens de serum periode en complete blootstelling werd voor de toekomstige experimenten gebruik gemaakt van de blootstelling van dag 3 tot dag 5 .

In hoofdstuk 3 wordt de ESTn verder gekarakteriseerd door genexpressie over de tijd te bestuderen met behulp van transcriptomics. We namen monsters van celkweken op dag 0 van de kweek (pluripotente ESC) en op de dagen 3, 4, 5, 6 en 7 tijdens de neurale differentiatie. Genen die gereguleerd waren over de tijd konden, op basis van hun genexpressie profiel, onderverdeeld worden in acht 
clusters, elk met een specifiek genexpressie patroon over de tijd. Met verscheidene analyse technieken kon aangetoond worden dat genen met een vroeg verhoogde genexpressie gerelateerd waren aan pluripotentie en transcriptie en dat laattijdig gereguleerde genen te maken hadden met neurale ontwikkeling. Daarnaast kon differentiatie over de tijd beschreven worden met behulp van de 'differentiatie track', waarbij door 'principal component analysis' het patroon van genexpressie van alle genen over de tijd weergegeven kan worden per monster. De 'differentiatie track' liet zien dat voor elk getest tijdspunt de monsters samen clusterden en dat deze groepjes chronologisch over de track verdeeld lagen. Daarnaast werd de variatie tussen monsters binnen een tijdsmeting groter bij monsters genomen op een later tijdspunt. Dit geeft aan dat cellen in de kweek differentieerden en ten opzichte van elkaar steeds meer verschillend werden.

Op eenzelfde manier werd ook het effect van $\mathrm{MeHg}$ bestudeerd met behulp van transcriptomics. Cellen werden blootgesteld aan $\mathrm{MeHg}$ vanaf dag 3, waarna monsters werden genomen na 24, 48, 72 en 96 uur blootstelling. Deze data lieten zien dat op elk tijdspunt $\mathrm{MeHg}$ de genexpressie verhoogde van neuraal gerelateerde genen en genexpressie verlaagde van genen gerelateerd aan algemene en vroege ontwikkeling. De 'differentiation track' techniek werd gebruikt om veranderingen van $\mathrm{MeHg}$ op de genexpressie aan te tonen na 24 , 48 en 72 uur blootstelling. MeHg monsters verschilden het meest van de 'differentiation track' na 24 uur blootstelling. Op 48 en 72 uur blootstelling was de variatie binnen de tijd en blootgestelde monsters te groot om een significant verschil op te kunnen pikken. Op basis van deze gegevens werden monsters voor de volgende transcriptomics experimenten blootgesteld voor 24 uur en, namen we controles mee van dag 3, 4 en 5 om de 'differentiatie track' op te kunnen zetten.

Een belangrijk gegeven in de toxicologie is dat giftigheid toeneemt met $\mathrm{d}$ concentratie van de blootstelling en dat er dus een concentratie hoort te zijn waarbij een stof niet giftig is. In hoofdstuk 4 gebruiken we transcriptomics om het effect van de blootstelling van een stof te bestuderen bij verschillende concentraties. Hiervoor gebruikten we drie stoffen waarvan bekend is dat ze ontwikkelingstoxisch zijn: de twee triazolen cyproconazole en hexaconazole en valproaat. Triazolen worden normaal gebruikt als antischimmel middelen en valproaat is een medicijn dat gebruikt wordt tegen epilepsie. Effecten van deze stoffen werden gemeten op genexpressie niveau na 24 uur blootstelling en vergeleken met de uitkomst van de morfologische score op dag 11. Alle drie de stoffen lieten een concentratie gemedieerd effect zien op genexpressie en biologische processen, wat informatie verschafte over mechanismen en con- centratierespons karakteristieken. Algemene en specifieke effecten van de verschillende stoffen konden zichtbaar gemaakt worden. Het biologische proces 'embryonic morphogenesis' werd bijvoorbeeld door elk van de stoffen beïnvloed, wat aangeeft dat deze stoffen een effect op 'standaard' ontwikkeling hebben. Verder werden voor cyproconazole en valproaat specifieke effecten op neurale ontwikkeling gevonden, terwijl deze niet gevonden werden voo hexaconazole. Dit komt overeen met de literatuur, aangezien cyproconazole en valproaat neuraal ontwikkelingstoxisch zijn en hexaconazole dat niet is. Verder konden klasse specifieke effecten worden gevonden voor de triazolen, aangezien zij het sterol biosynthese proces beïnvloedden, een belangrijk mechanisme in de antischimmel werking van deze stoffen. Het gebruik van transcriptomics in deze studie toonde verder aan dat effecten op genexpressie eerder waarneembaa waren dan effecten op morfologie, waardoor dit een meer gevoeligere methode kan zijn om ontwikkelingstoxiciteit te meten.

In de volgende transcriptomics studie in hoofdstuk 5 werden effecten op genexpressie van zes stoffen met verschillende werkingsmechanismen bestudeerd, om het toepassingsdomein van het testsysteem te bepalen. Vijf van deze stoffen zijn bekende ontwikkelingstoxische stoffen en één stof was meegenomen als een negatieve controle. De stoffen werden getest op een toxische concentratie waarop het effect op morfologie vergelijkbaar was tussen de stoffen én een concentratie die 30 keer lager, en dus niet toxisch, was. Sto specifieke en algemene genexpressie veranderingen werden waargenomen tussen de verschillende stoffen. Er werden echter geen processen gevonden die door alle ontwikkelingstoxische stoffen aangedaan waren. Dit toont aan dat effecten op neurale differentiatie via meerdere mechanismen geïnduceerd kunnen worden. Ook konden genexpressie profielen en proces verrijking gebruikt worden om de verschillen in genexpressie in een niet toxische en ontwikkelingstoxische concentratie te vergelijken. Deze informatie kan gebruikt worden om verschillen in genexpressie veranderingen ten opzichte van adaptieve of ongunstige effecten te verklaren.

In hoofdstuk 6 zijn de transcriptomics data van hoofdstukken 3, 4 en 5 gecombineerd om een biomarker genen set te verkrijgen om neurale ontwikkelingstoxiciteit in de ESTn te voorspellen. Genexpressie veranderingen werden vergeleken met behulp van de 'differentiatie track' waarbij data van 10 stoffen en 19 blootstellingen werd gebruikt. Met behulp van een genen set evaluatie gekoppeld aan een 'leave-one-out cross-validatie' kon een set van 29 genen gevonden worden die vooral te maken hadden met neurale ontwikkeling. Met deze genen set kon neurale ontwikkelingstoxiciteit in de ESTn voor $84 \%$ van 
de blootstellingen goed voorspeld worden. Deze set verbetert de predictie van de ESTn en kan in de toekomst eventueel gebruikt worden voor risico evaluaties.

De ESTn was ontwikkeld om gebruikt te worden in combinatie met de originele ESTc. De overeenkomsten en verschillen tussen de twee modellen op genexpressie niveau worden beschreven in hoofdstuk 7. Tijd gereguleerde genexpressie profielen van ESTn en ESTc op dag 3, 4 en 5 en blootstelling aan zeven stoffen werden tussen de modellen vergeleken. Hoewel genexpressie verschillen over de tijd tussen de twee protocollen voor een groot dee vergelijkbaar waren, was er een kleinere groep genen die zeer specifiek gereguleerd werd door één van beide modellen en niet in het andere model. Deze genen waren gerelateerd aan hartspierdifferentiatie in de ESTc en neurale differentiatie in ESTn. Stof geïnduceerde genexpressie veranderingen ware over het algemeen zeer verschillend tussen de modellen, waardoor de ontwikkelingstoxiciteit óf in de ESTc (bijvoorbeeld flusilazole) óf in de ESTn (bijvoorbeeld $\mathrm{MeHg}$ ) goed werd voorspeld. Een uitzondering hierop was valproaat, waarbij genexpressie veranderingen in beide modellen vergelijkbaar waren, hoewel er op het proces niveau grote verschillen waren. Een vergelijking op genexpressie niveau tussen ESTn en ESTc laat zien dat beide modellen een eigen specifieke bijdrage kunnen leveren aan voorspelling van ontwikkelingstoxiciteit.

Een vergelijking tussen ESTn en andere in vitro en in vivo modellen op transcriptomics niveau is uitgevoerd in hoofdstuk 8 , om inzicht te krijgen in overeenkomsten en verschillen van mechanismen in deze modellen. Het effect van $\mathrm{MeHg}$ op genexpressie in verschillende in vitro en in vivo modellen was bestudeerd. Modellen voor ontwikkelingstoxiciteit waren de in vitro rat 'whole embryo culture', ESTc en ESTn en twee in vivo embryotoxiciteitstesten. Daarnaast waren modellen vergeleken welke acute toxiciteit voorspellen zoals een embryonale fibroblast cellijn en het jong-volwassen brein en de nier. Evaluatie van vergelijkbare en unieke aspecten op het functionele en genexpressie niveau toonden aan dat deze een vergelijkbaar patroon lieten zien in de ontwikkelingstoxiciteit modellen, maar niet in de andere modellen. Deze vergelijking laat zien dat extrapolatie van in vitro naar in vivo modellen relevant is en dat genexpressie veranderingen over modellen heen voor een groot deel overeen komen.

\section{Conclusie}

$\mathrm{N}$ dit proefschrift wordt de ontwikkeling van de ESTn en implementatie van 1 transcriptomics in de ESTn test strategie beschreven. Dit leidde uiteindelijk tot een biomarker genen set voor het voorspellen van neurale ontwikkelingstoxiciteit in de ESTn. Stof effecten op genexpressie over tijd en concentratie zijn beschreven en bestudeerd, waardoor een vergelijking tussen mechanismen van verschillende neuraal ontwikkelingstoxische stoffen gemaakt kan worden. Verder is er een vergelijking gemaakt tussen de ESTn, ESTc en andere in vitro en in vivo modellen waaruit blijkt dat de ESTn een bijdrage kan leveren aan mechanistisch inzicht en een verhoging van de predictie in ontwikkelingstoxiciteit test strategieën. Om de predictie van de ESTn verder te verhogen is het nodig om het toepassingsdomein van de ESTn verder te definiëren, waarbij he belangrijk is om de mechanismen achter toxiciteit in de ESTn in kaart te brengen.

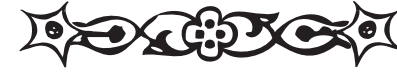




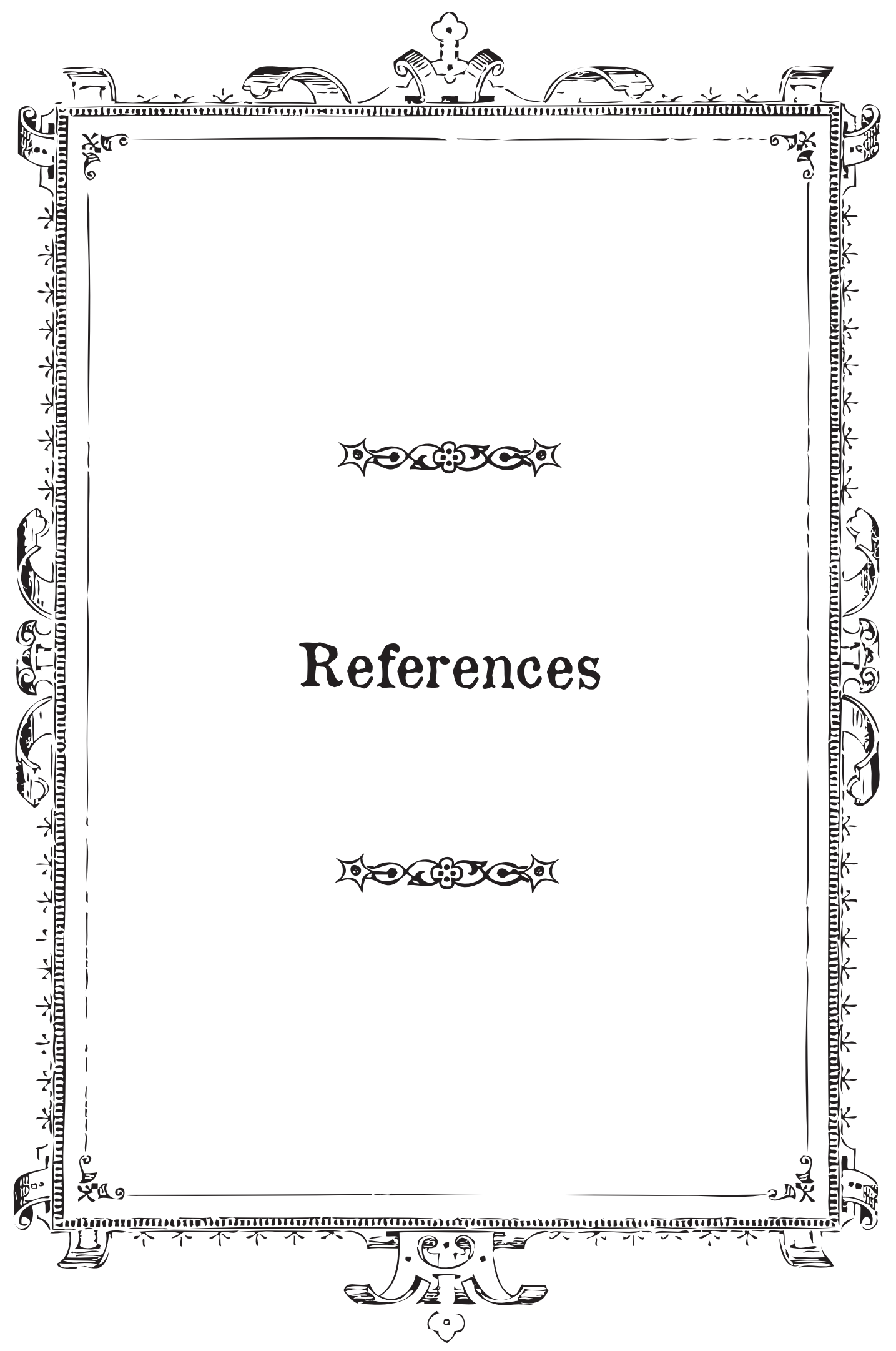




\section{References}

1. Hale, F., Relation of Vitamin A to Anophtalmos in Pigs. Am. J. Ophth., 1935(18): p. 1087.

2. Warkany, J. and E. Schraffenberge, Congenital malformations of the eyes induced in rats by maternal vitamin A deficiency. Proc. Soc. Exp. Biol. Med., 1944(57): p. 49-52.

3. Cohlan, S., Congenital anomalies in the rat produced by excessive intake of vitamin $A$ during pregnancy. Pediatrics, 1954(13): p. 556-567.

4. Gregg, N.M.A., Congenital cataract following German measles in the mother. Trans. Ophthalmol. Soc. Australia, 1941(3): p. 35-46.

5. Harada, Y., Congenital (or fetal) Minamata disease, in Minamata Disease, S.g.o.M Disease, Editor. 1968, Kumamotot University, Japan.

6. Lenz, W., A short history of thalidomide embryopathy. Teratology, 1988. 38(3): p. 203-15.

7. Wilson, J., Environmental Birth Defects, ed. J. Wilson. 1973, London: Acadamic Pr.

8. Goldenthal, E., Guidelines for Reproduction studies for safety evaluation of drugs for buman use, B.o.S. FDA, Editor. March 1 1966, Drug review branch, division of toxicological evaluation, bureau of science, U.S. Food and Drug Administartion: Silver Spring, MD.

9. ICH, Step 4 tripartite harmonised guidelines. Detection of toxicity to reproductiion for medicinal products. Proceedings of the second international conference on harmonisation Orlando., ed. P. D’Arcy and D. Haron. 1994, Belfast: Queen's University.

10. Barrow, P.C., Reproductive toxicity testing for pharmaceuticals under ICH. Reprod Toxicol, 2009. 28(2): p. 172-9.

11. OECD, Draft guidance document on reproductive toxicity testing and assessment, H.a.S.D. Environment, Editor. 2004, OECD: Paris.

12. van der Jagt, K., J. Tørsløv, and J. de Bruijn, Alternative approaches can reduce the use of test animals under REACH. European Commission Report. 2004.

13. Hartung, T and C Rovida, Chemical regulators have overreached. Nature, 2009. 460(7259): p. 1080-1.

14. Piersma, A.H., et al., Validation of the postimplantation rat whole-embryo culture test in the international ECVAM validation study on three in vitro embryotoxicity tests. Altern Lab Anim, 2004. 32(3): p. 275-307.

5. de Jong, E., M. Barenys, S.A. Hermsen, A. Verhoef, B.C. Ossendorp, J.G. Bessems, and A.H. Piersma, Comparison of the mouse Embryonic Stem cell Test, the rat Whole Embryo Culture and the Zebrafish Embryotoxicity Test as alternative methods for development toxicity testing of six 1,2,4-triazoles. Toxicol Appl Pharmacol, 2011. 253(2): p. 103-11.

16. Nagel, R., DarT: The embryo test with the Zebrafish Danio rerio--a general model in ecotoxicology and toxicology. ALTEX, 2002. 19 Suppl 1: p. 38-48.

17. Genschow, E., H. Spielmann, G. Scholz, I. Pohl, A. Seiler, N. Clemann, S. Bremer, and K. Becker, Validation of the embryonic stem cell test in the international ECVAM validatio study on three in vitro embryotoxicity tests. Altern Lab Anim, 2004. 32(3): p. 209-44.

18. Spielmann, H., I. Pohl, B. Döring, M. Liebsch, and F. Moldenhauer, The embryonic stem cell test (EST), an in vitro embryotoxicity test using two permanent mouse cell lines: $3 T 3$ fibroblasts and embryonic stem cells. In Vitro Toxicology, 1997. 10: p. 119-27.

19. Keller, G., Embryonic stem cell differentiation: emergence of a new era in biology and medicine. Genes Dev, 2005. 19(10): p. 1129-55.

20. Rudnicki, M. and M. McBurney, Cell culture methods and induction of differentiation of embryonal carcinoma cell lines, in Teratocarcinoma and embryonic stem cells: a practical approach, E. Robertson, Editor. 1987, IRL Press: Washington D.C. p. 19-49.

21. Martin, G.R. and M.J. Evans, Differentiation of clonal lines of teratocarcinoma cells: formation of embryoid bodies in vitro. Proc Natl Acad Sci U S A, 1975. 72(4): p. 1441-5.

22. Maximow, A., Der Lymphozyt als gemeinsame Stammzelle der verschiedenen Blutelemente in der embryonalen Entwicklung und im postfetalen Leben der Saugetiere. Folia Haematologica, 1909. 8: p. 125-134. 
23. Till, J. and E. McCulloch, A direct measurement of the radiation sensitivity of normal mouse bone marrow cells. Radiation Research, 1961 14: p. 213-22.

24. Becker, A.J., E. McCulloch, and J. Till, Cytological demonstration of the clonal nature of spleen colonies derived from transplanted mouse marrow cells. Nature, 1963. 197: p. 452-4. . Kahan, B. and B. Ephrussi, Developmental potentialities of clonal in vitro cultures of mouse testicular teratoma. J Natl Cancer Inst., 1970. 44: p. 1015-36,

26. Martin, G. and M. Evans, The morphology and growth of a pluripotent teratocarcinom cell line and its derivatives in tissue culture. Cell, 1974. 2(163-72).

7. Hsu, Y. and J. Baskar, Differentiation in vitro of normal mouse embryos and mouse 53(1): p. $177-85$.

8. Murmmery, C.L., T.A. van Achterberg, A.J. van den Eijnden-van Raaij, L. van Haaster, A. Willemse, S.W. de Laat, and A.H. Piersma, Visceral-endoderm-like cell lines induce differentiation of murine P1O embryonal carcinoma cells. Differentiation, 1991. 46(1): p. 51-60.

29. Piersma, A.H., A.S. Haakmat, and A.M. Hagenaars, In vitro assays for the developmental toxicity of xenobiotic compounds using differentiating embryonal carcinoma cells culture. Toxicol In Vitro, 1993. 7(5): p. 615-21.

30. Wobus, A.M. and K.R. Boheler, Embryonic stem cells: prospects for developmental biology and cell therapy. Physiol Rev, 2005. 85(2): p. 635-78.

1. Evans, M.J. and M.H. Kaufman, Establishment in culture of pluripotential cells from mouse embryos. Nature, 1981. 292(5819): p. 154-6.

32. Martin, G.R., Isolation of a pluripotent cell line from early mouse embryos cultured in medium conditioned by teratocarcinoma stem cells. Proc Natl Acad Sci U S A, 1981. 78(12), p. 7634-8.

33. Thomson, J.A., J. Itskovitz-Eldor, S.S. Shapiro, M.A. Waknitz, J.J. Swiergiel, V.S. Marshall, and J.M. Jones, Embryonic stem cell lines derived from human blastocysts. Science, 1998. 282(5391): p. 1145-7.

34. Scholz, G., E. Genschow, I. Pohl, S. Bremer, M. Paparella, H. Raabe, J. Southee, and H. Spielmann, Prevalidation of the Embryonic Stem Cell Test (EST)-A New In Vitro Embryotoxicity Test. Toxicol In Vitro, 1999. 13(4-5): p. 675-81.

35. Hartung, T., et al., A modular approach to the ECVAM principles on test validity. Altern Lab Anim, 2004. 32(5): p. 467-72.

36. Spielmann, H., et al., The practical application of three validated in vitro embryotoxicity tests. The report and recommendations of an ECVAM/ZEBET workshop (ECVAM workshop 57). Altern Lab Anim, 2006. 34(5): p. 527-38.

37. Marx-Stoelting, P., et al., A review of the implementation of the embryonic stem cell test EST). The report and recommendations of an ECVAM/ReProTect Workshop. Altern Lab Anim, 2009. 37(3): p. 313-28.

38. De Smedt, A., M. Steemans, M. De Boeck, A.K. Peters, B.J. van der Leede, F. Van Goethem, A. Lampo, and P. Vanparys, Optimisation of the cell cultivation methods in the embryonic stem cell test results in an increased differentiation potential of the cells into strong beating myocard cells. Toxicol In Vitro, 2008. 22(7): p. 1789-96.

39. Stummann, T.C., L. Hareng, and S. Bremer, Embryotoxicity hazard assessment of cadmium and arsenic compounds using embryonic stem cells. Toxicology, 2008. 252(1-3): p. 118-22.

40. van Dartel, D.A., J.L. Pennings, P.J. Hendriksen, F.J. van Schooten, and A.H. Piersma, Early gene expression changes during embryonic stem cell differentiation into cardiomyocytes and their modulation by monobutyl phthalate. Reprod Toxicol, 2009. 27(2): p. 93-102.

1. Stummann, T.C., L. Hareng, and S. Bremer, Embryotoxicity hazard assessment of methylmercury and chromium using embryonic stem cells. Toxicology, 2007. 242(1-3): p. 130-43.

2. zur Nieden, N.I., L.A. Davis, and D.E developmental osteotoxicity in the embryonic stem cell test. Tor 247(2): p. $91-7$.
43. Eriksson, P., Developmental neurotoxicity of environmental agents in the neonate. Neurotoxicology, 1997. 18(3): p. 719-26

44. Rice, D. and S. Barone, Jr., Critical periods of vulnerability for the developing nervous system: evidence from humans and animal models. Environ Health Perspect, 2000. 108 Suppl 3: p. 511-33.

45. Mendola, P., S.G. Selevan, S. Gutter, and D. Rice, Environmental factors associated with spectrum of neurodevelopmental deficits. Ment Retard Dev Disabil Res Rev, 2002. 8(3): p. 188-97.

46. OECD, Report of the OECD Ad Hoc Working Group on Reproduction and Developmental Toxicity. 1995: Copenhagen, Denmark. p. 13 .

47. Harrison, $R$, Observations on the living developing nerve fiber. Anat. Rec., 1907. 1: p. 116-128.

48. Harrison, R., The outgrowth of the nerve fiber as a mode of protoplasmic movement. J. Exp. Zool., 1910. 9: p. 787-846.

9. Altman, J. and G. Das, Postnatal neurogenesis in the guinea-pig. Nature, 1967. 214(5093): p. 1098-101.

0. Greene, L. and A. Tischler, Establishment of a noradrenergic clonal line of rat adrenal pheochromocytoma cells which respond to nerve growth factor. Proc. Natl. Acad. Sci. U.S.A., 1976. 73(7): p. 2424-8,

51. Reynolds, B. and S. Weiss, Generation of neurons and astrocytes from isolated cells of the adult mammalian central nervous system. Science, 1992. 255(5052): p. 1707-10.

52. Okabe, S., K. Forsberg-Nilsson, A.C. Spiro, M. Segal, and R.D. McKay, Development of neuronal precursor cells and functional postmitotic neurons from embryonic stem cells in vitro. Mech Dev, 1996. 59(1): p. 89-102.

33. Zhang, S., M. Wernig, I. Duncan, O. Brustle, and J. Thomson, In vitro differentiation of transplantable neural precursors from human embryonic stem cells. Nat Biotechnol, 2001 19(12): p. 1129-33.

54. Bain, G., W.J. Ray, M. Yao, and D.I. Gottlieb, Retinoic acid promotes neural and represses mesodermal gene expression in mouse embryonic stem cells in culture. Biochem Biophy Res Commun, 1996. 223(3): p. 691-4.

55. Mummery, C.L., C.E. van den Brink, P.T. van der Saag, and S.W. de Laat, A short-term screening test for teratogens using differentiating neuroblastoma cells in vitro. Teratology, 1984. 29(2): p. 271-9.

56. Cai, C. and L. Grabel, Directing the differentiation of embryonic stem cells to neural stem cells. Dev Dyn, 2007. 236(12): p. 3255-66.

57. Worth, A.P. and M. Balls, The principles of validation and the ECVAM validation process. Altern Lab Anim, 2002. 30 Suppl 2: p. 15-21.

58. Rodier, P.M., Vulnerable periods and processes during central nervous system development. Environ Health Perspect, 1994. 102 Suppl 2: p. 121-4.

59. Cowan, W., Molecular and cellular approaches to neural development. 1997: Oxfor University Press. 563

60. Bal-Price, A.K., H.T. Hogberg, L. Buzanska, and S. Coecke, Relevance of in vitro neurotoxicity testing for regulatory requirements: challenges to be considered. Neurotoxicol Teratol, 2010. 32(1): p. 36-41.

61. Coecke, S., et al., Workgroup report: incorporating in vitro alternative methods for hazard and risk assessment strategies. Environ Health Perspect, 2007. 115(6): p. 924-31.

62. Kuegler, P.B., et al., Markers of murine embryonic and neural stem cells, neurons and astrocytes: reference points for developmental neurotoxicity testing. ALTEX, 2010. 27(1):

D. and F.H. Crick, The structure of DNA. Cold Spring Harb Symp Quant Biol, 1953. 18: p. 123-31. 
64. Chee, M., et al., Accessing genetic information with high-density DNA arrays. Science, 1996. 274(5287): p. 610-4

65. Lander, E.S., et al., Initial sequencing and analysis of the human genome. Nature, 2001. 409(6822): p. 860-921.

66. Nuwaysir, E.F., M. Bittner, J. Trent, J.C. Barrett, and C.A. Afshari, Microarrays and toxicology: the advent of toxicogenomics. Mol Carcinog, 1999. 24(3): p. 153-9.

67. Kramer, J.A., S.W. Curtiss, K.L. Kolaja, C.L. Alden, E.A. Blomme, W.C. Curtiss, J.C. Davila, C.J. Jackson, and R.T. Bunch, Acute molecular markers of rodent hepatic carcinogenesis identifed

68. Shi, L., et al., The MicroArray Quality Control (MAQC) project shows inter- and intraplatform reproducibility of gene expression measurements. Nat Biotechnol, 2006. 24(9): p. 1151-61.

69. Iguchi, T., H. Watanabe, and Y. Katsu, Toxicogenomics and ecotoxicogenomics for studying endocrine disruption and basic biology. Gen Comp Endocrinol, 2007. 153(1-3): p. 25-9.

70. Baken, K.A., R.J. Vandebriel, J.L. Pennings, J.C. Kleinjans, and H. van Loveren, Toxicogenomics in the assessment of immunotoxicity. Methods, 2007. 41(1): p. 132-41.

1. Yang, Y., E.A. Blomme, and J.F. Waring, Toxicogenomics in drug discovery: from preclinical studies to clinical trials. Chem Biol Interact, 2004. 150(1): p. 71-85.

72. Daston, G.P. and J.M. Naciff, Predicting developmental toxicity through toxicogenomics. Birth Defects Res C Embryo Today, 2010. 90(2): p. 110-7.

73. Naciff, J.M., et al., Gene expression changes induced in the testis by transplacental exposure to high and low doses of 17\{alpha\}-ethynyl estradiol, genistein, or bisphenol A. Toxicol Sci, 2005. 86(2): p. 396-416

74. Naciff, J.M., S.M. Torontali, G.I. Overmann, G.J. Carr, J.P. Tiesman, and G.P. Daston, Evaluation of the gene expression changes induced by 17-alpha-ethynyl estradiol in the immature uterus/ovaries of the rat using high density oligonucleotide arrays. Birth Defects Res B Dev Reprod Toxicol, 2005. 74(2): p. 164-84.

75. Goetz, A.K. and D.J. Dix, Mode of action for reproductive and hepatic toxicity inferred from a genomic study of triazole antifungals. Toxicol Sci, 2009. 110(2): p. 449-62.

76. Jergil, M., K. Kultima, A.L. Gustafson, L. Dencker, and M. Stigson, Valproic acid-induced deregulation in vitro of genes associated in vivo with neural tube defects. Toxicol Sci, 2009. 108(1): p. $132-48$

77. Robinson, J.F., A. Verhoef, J.L. Pennings, T.E. Pronk, and A.H. Piersma, A comparison of gene expression responses in rat whole embryo culture and in vivo: time-dependent retinoic acid-induced teratogenic response. Toxicol Sci, 2012. 126(1): p. 242-54.

78. Hermsen, S.A., E.J. van den Brandhof, L.T. van der Ven, and A.H. Piersma, Relative mbryotoxicity of two classes of chemicals in a modified zebrafish embryotoxicity test and comparison with their in vivo potencies. Toxicol In Vitro, 2011.

79. van Dartel, D.A. and A.H. Piersma, The embryonic stem cell test combined with toxicogenomics as an alternative testing model for the assessment of developmental toxicity. Reprod Toxicol, 2011. 32(2): p. 235-44.

80. Zimmer, B., et al., Coordinated waves of gene expression during neuronal differentiation of embryonic stem cells as basis for novel approaches to developmental neurotoxicity testing. Cell Death Differ, 2010.

81. van Dartel, D.A., J.L. Pennings, J.F. Robinson, J.C. Kleinjans, and A.H. Piersma, Discriminating classes of developmental toxicants using gene expression profiling in the embryonic stem cell test. Toxicol Lett, 2011. 201(2): p. 143-51.

82. Robinson, J.F., V.A. van Beelen, A. Verhoef, M.F. Renkens, M. Luijten, M.H. van Herwijnen, Westerman, Ju. Pennings, and A.H. Piersma, Embryotoxicant-specific transcriptomic A. Wesponses in rat postimplantation whele-embryo calture. Toxicol Sci, 2010. 118(2): p. 675-85.
83. van Dartel, D.A., J.L. Pennings, L.J. de la Fonteyne, M.H. van Herwijnen, J.H. van Delft, F.J. van Schooten, and A.H. Piersma, Monitoring developmental toxicity in the embryonic stem cell test using differential gene expression of differentiation-related genes. Toxicol Sci, 2010. 116(1): p. 130-9.

84. Pennings, J.L., D.A. van Dartel, J.F. Robinson, T.E. Pronk, and A.H. Piersma, Gene set assembly for quantitative prediction of developmental toxicity in the embryonic stem cell test. Toxicology, 2011. 284(1-3): p. 63-71.

85. Banjamini, Y. and Y. Hochberg, Controlling the false discovery rate: A practical and powerful approach to multiple testing. J.R. Statist. Soc. B, 1995. 57: p. 289-300.

86. Pearson, K., On lines and planes of closest fit to systems of points in space. Philos. Mag., 1901. 2: p. 559-72

87. Eisen, M.B., P.T. Spellman, P.O. Brown, and D. Botstein, Cluster analysis and display of genome-wide expression patterns. Proc Natl Acad Sci U S A, 1998. 95(25): p. 14863-8.

8. Hayes, K.R. and C.A. Bradfield, Advances in toxicogenomics. Chem Res Toxicol, 2005. 18(3): p. 403-14

89. Mattingly, C.J., G.T. Colby, J.N. Forrest, and J.L. Boyer, The Comparative Toxicogenomic Database (CTD). Environ Health Perspect, 2003. 111(6): p. 793-5.

9. Rebhan, M., V. Chalifa-Caspi, J. Prilusky, and D. Lancet, GeneCards: a novel functional genomics compendium with automated data mining and query reformulation support. Bioinformatics, 1998. 14(8): p. 656-64

91. Sayers, E.W., et al., Database resources of the National Center for Biotechnology Information. Nucleic Acids Res, 2009. 37(Database issue): p. D5-15.

92. Huang da, W., B.T. Sherman, and R.A. Lempicki, Systematic and integrative analysis of large gene lists using DAVID bioinformatics resources. Nat Protoc, 2009. 4(1): p. 44-57.

93. Kelder T, Pico AR, Hanspers K, van Iersel MP, Evelo C, and C. BR, Mining Biological Pathways Using WikiPathways Web Services. PLoS ONE, 2009. 4(7).

94. Huang da, W., et al., The DAVID Gene Functional Classification Tool: a novel biological module-centric algorithm to functionally analyze large gene lists. Genome Biol, 2007. 8(9): p. R183.

95. Yu, X., W.C. Griffith, K. Hanspers, J.F. Dillman, 3rd, H. Ong, M.A. Vredevoogd, and E.M. Faustman, A system-based approach to interpret dose- and time-dependent microarray data: quantitative integration of gene ontology analysis for risk assessment. Toxicol Sci, 2006. $92(2):$ p. 560-77

96. Dahlquist, K.D., N. Salomonis, K. Vranizan, S.C. Lawlor, and B.R. Conklin, GenMAPP, new tool for viewing and analyzing microarray data on biological pathways. Nat Genet, 2002. 31(1): p. 19-20.

97. Nikolsky, Y., S. Ekins, T. Nikolskaya, and A. Bugrim, A novel method for generation of signature networks as biomarkers from complex high throughput data. Toxicol Lett, 2005. $158(1)$ : p. $20-9$.

98. Martinkova, P., K. Zvara, Jr., J. Zvarova, and K. Zvara, The new features of the ExaMe evaluation system and reliability of its fixed tests. Methods Inf Med, 2006. 45(3): p. 310-5.

99. Boorsma, A., B.C. Foat, D. Vis, F. Klis, and H.J. Bussemaker, T-profiler: scoring the activit of predefined groups of genes using gene expression data. Nucleic Acids Res, 2005.33(Web Server issue): p. W592-5.

100. Subramanian, A, et al, Gene set enrichment analysis: a knowledge-based approach for interpreting genome-wide expression profiles. Proc Natl Acad Sci US A, 2005.102(43): 15545-50.

101. Daston, G.P., R.E. Chapin, A.R. Scialli, A.H. Piersma, E.W. Carney, J.M. Rogers, and J.M. Friedman, A different approach to validating screening assays for developmental toxicity. Birth Defects Res B Dev Reprod Toxicol, 2010. 89(6): p. 526-30.

102. van der Jagt, K.M., S; Tørsløv, J; de Bruijn, J, Alternative approaches can reduce the use of test animals under REACH. European Commission Report., 2004: p. EUR 21405 EN. 
103. Bibel, M., J. Richter, K. Schrenk, K.L. Tucker, V. Staiger, M. Korte, M. Goetz, and Y.A. Barde, Differentiation of mouse embryonic stem cells into a defined neuronal lineage. Nat Barde, Differentiation of mouse
Neurosci, 2004. 7(9): p. 1003-9.

104. Suter, D. and K.H. Krause, Neural commitment of embryonic stem cells: molecules, pathways and potential for cell therapy. J Pathol, 2008. 125(4): p. $355-368$.

105. Ying, Q.L. and A.G. Smith, Defined conditions for neural commitment and differentiation. Methods Enzymol, 2003. 365: p. 327-41.

106. Fico, A., G. Manganelli, M. Simeone, S. Guido, G. Minchiotti, and S. Filosa, High-throughput screening-compatible single-step protocol to differentiate embryonic stem cells in neurons. Stem Cells Dev, 2008. 17(3): p. 573-84

107. OECD, OECD Guidelines for the Testing of Chemicals / Section 4: Health Effects Test No. 426: Developmental Neurotoxicity Study 2007, Paris: OECD. 26.

108. Kimmel, C.A., Rees, D.C., Francis, E.Z., Qualitative and quantitative comparability of human and animal developmental neurotoxicity. Williamsburg, Virginia, April 11-13, 1989.Proceedings. Neurotoxicol Teratol, 1990. 12(3): p. 173-292.

109. Davidson, P.W., G.J. Myers, and B. Weiss, Mercury exposure and child development outcomes. Pediatrics, 2004. 113(4 Suppl): p. 1023-9.

10. Miller, M.W. and G. Potempa, Numbers of neurons and glia in mature rat somatosensor cortex: effects of prenatal exposure to ethanol. J Comp Neurol, 1990. 293(1): p. 92-102.

11. Cox, C., T.W. Clarkson, D.O. Marsh, L. Amin-Zaki, S. Tikriti, and G.G. Myers, Dose-response analysis of infants prenatally exposed to methyl mercury: an application of a single compartment model to single-strand hair analysis. Environ Res, 1989. 49(2): p. 318-32.

12. Pevny, L.H., S. Sockanathan, M. Placzek, and R. Lovell-Badge, A role for SOX1 in neural determination. Development, 1998. 125(10): p. 1967-78.

13. Walther, C. and P. Gruss, Pax-6, a murine paired box gene, is expressed in the developing CNS. Development, 1991. 113(4): p. 1435-49.

14. Frederiksen, K. and R.D. McKay, Proliferation and differentiation of rat neuroepithelial precursor cells in vivo. J Neurosci, 1988. 8(4): p. 1144-51

115. Strubing, C., G. Ahnert-Hilger, J. Shan, B. Wiedenmann, J. Hescheler, and A.M. Wobus, Differentiation of pluripotent embryonic stem cells into the neuronal lineage in vitro gives rise to mature inhibitory and excitatory neurons. Mech Dev, 1995. 53(2): p. 275-87.

116. Fujikura, J., E. Yamato, S. Yonemura, K. Hosoda, S. Masui, K. Nakao, J. Miyazaki Ji, and H. Niwa, Differentiation of embryonic stem cells is induced by GATA factors. Genes Dev, 2002. 16(7): p. 784-9.

117. Tomasi, T.B., Jr., Structure and function of alpha-fetoprotein. Annu Rev Med, 1977. 28: p. 453-65.

118. Kwon, G.S., S.T. Fraser, G.S. Eakin, M. Mangano, J. Isern, K.E. Sahr, A.K. Hadjantonakis, and M.H. Baron, $\operatorname{Tg}(A f p-G F P)$ expression marks primitive and definitive endoderm lineages during mouse development. Dev Dyn, 2006. 235(9): p. 2549-58.

119. Duncan, S.A., K. Manova, W.S. Chen, P. Hoodless, D.C. Weinstein, R.F. Bachvarova, and E. Darnell, Jr., Expression of transcription factor HNF-4 in the extraembryonic endoderm, gut, and nephrogenic tissue of the developing mouse embryo: HNF-4 is a marker for . 7598-602.

120. Wilkinson, D.G., S. Bhatt, and B.G. Herrmann, Expression pattern of the mouse T gene and its role in mesoderm formation. Nature, 1990. 343(6259): p. 657-9.

121. Lyons, I., L.M. Parsons, L. Hartley, R. Li, J.E. Andrews, L. Robb, and R.P. Harvey, Myogenic and morphogenetic defects in the heart tubes of murine embryos lacking the bomeo box gene Nkx2-5. Genes Dev, 1995. 9(13): p. 1654-66

122. Mahdavi, V., M. Periasamy, and B. Nadal-Ginard, Molecular characterization of two myosin heavy chain genes expressed in the adult heart Nature, 1982. 297(5868): p. 659-64.
23. Bai, L., H. Xu, J.F. Collins, and F.K. Ghishan, Molecular and functional analysis of a novel neuronal vesicular glutamate transporter. J Biol Chem, 2001. 276(39): p. 36764-9.

124. McIntire, S.L., R.J. Reimer, K. Schuske, R.H. Edwards, and E.M. Jorgensen, Identification and characterization of the vesicular GABA transporter. Nature, 1997. 389(6653): p. 870-6. 125. Erlander, M.G., N.J. Tillakaratne, S. Feldblum, N. Patel, and A.J. Tobin, Two genes encode distinct glutamate decarboxylases. Neuron, 1991. 7(1): p. 91-100.

126. Grima, B., A. Lamouroux, F. Blanot, N.F. Biguet, and J. Mallet, Complete coding sequence of rat tyrosine hydroxylase mRNA. Proc Natl Acad Sci U S A, 1985. 82(2): p. 617-21.

127. Ninkina, N.N., G.E. Stevens, J.N. Wood, and W.D. Richardson, A novel Brn3-like POU transcription factor expressed in subsets of rat sensory and spinal cord neurons. Nucleic Acids Res, 1993. 21(14): p. 3175-82

128. Vult von Steyern, F., V. Martinov, I. Rabben, A. Nja, O. de Lapeyriere, and T. Lomo, The homeodomain transcription factors Islet 1 and HB9 are expressed in adult alpha an gamma motoneurons identified by selective retrograde tracing. Eur J Neurosci, 1999. 11(6) p. 2093-102.

129. Pekny, M., P. Leveen, M. Pekna, C. Eliasson, C.H. Berthold, B. Westermark, and C. Betsholtz, Mice lacking glial fibrillary acidic protein display astrocytes devoid of intermediate filaments but develop and reproduce normally. Embo J, 1995. 14(8): p. 1590-8.

130. Tamm, C., J. Duckworth, O. Hermanson, and S. Ceccatelli, High susceptibility of neural stem cells to methylmercury toxicity: effects on cell survival and neuronal differentiation. Neurochem, 2006. 97(1): p. 69-78.

131. Buzanska, L., J. Sypecka, S.N. Molteni, A. Compagnoni, H.T. Hogberg, R. Del Torchio, K. Domanska-Janik, J. Zimmer, and S. Coecke, A Human Stem Cell Based Model For Identifying Adverse Effects Of Organic And Inorganic Chemicals On The Developing Nervous System. Stem Cells, 2009.

132. Moors, M., T.D. Rockel, J. Abel, J.E. Cline, K. Gassmann, T. Schreiber, J. Schuwald, N. Weinmann, and E. Fritsche, Human neurospheres as three-dimensional cellular systems for developmental neurotoxicity testing. Environ Health Perspect, 2009. 117(7): p. 1131-8.

133. Maric, D., Q.Y. Liu, I. Maric, S. Chaudry, Y.H. Chang, S.V. Smith, W. Sieghart, J.M. Fritschy, and J.L. Barker, GABA expression dominates neuronal lineage progression in the embryonic rat neocortex and facilitates neurite outgrowth via GABA(A) autoreceptor/Clchannels. J Neurosci, 2001. 21(7): p. 2343-60.

134. Jelitai, M. and E. Madarasz, The role of GABA in the early neuronal development. Int Rev Neurobiol, 2005. 71: p. 27-62.

135. Wojcik, S.M., J.S. Rhee, E. Herzog, A. Sigler, R. Jahn, S. Takamori, N. Brose, and C. Rosenmund, An essential role for vesicular glutamate transporter 1 (VGLUT1) in postnatal development and control of quantal size. Proc Natl Acad Sci U S A, 2004. 101(18): p. 7158-63.

136. Miyazaki, T., M. Fukaya, H. Shimizu, and M. Watanabe, Subtype switching of vesicular glutamate transporters at parallel fibre-Purkinje cell synapses in developing mouse cerebellum. Eur J Neurosci, 2003. 17(12): p. 2563-72.

137. van Dartel, D.A., N.J. Zeijen, L.J. de la Fonteyne, F.J. van Schooten, and A.H. Piersma, Disentangling cellular proliferation and differentiation in the embryonic stem cell test, and its impact on the experimental protocol. Reprod Toxicol, 2009. 28(2): p. 254-61.

138. Stummann, T.C., L. Hareng, and S. Bremer, Hazard assessment of methylmercury toxicity to neuronal induction in embryogenesis using human embryonic stem cells. Toxicology, 2009. 257(3): p. 117-26.

139. Radio, N.M., J.M. Breier, T.J. Shafer, and W.R. Mundy, Assessment of chemical effects on Wening. Toxicol Sci, 2008 105(1): 106-18

140. Gotz M.E. E. Koutsilieri, P. Riederer, S. Ceccatelli, and E. Dare, Methylmercury induces neurite degeneration in primary culture of mouse dopaminergic mesencephalic cells. J Neural Transm, 2002. 109(5-6): p. 597-605. 
141. Farah, C.A. and N. Leclerc, HMWMAP2: new perspectives on a pathway to dendritic identity. Cell Motil Cytoskeleton, 2008. 65(7): p. 515-27.

142. Theunissen, P.T., S.H. Schulpen, D.A. van Dartel, S.A. Hermsen, F.J. van Schooten, and A.H. Piersma, An abbreviated protocol for multilineage neural differentiation of murine embryonic stem cells and its perturbation by methyl mercury. Reprod Toxicol, 2010. 29(4): p. 383-92.

143. Alsdorf, R. and D.F. Wyszynski, Teratogenicity of sodium valproate. Expert Opin Drug Saf, 2005. 4(2): p. 345-53.

144. Faustman, E.M., R.A. Ponce, Y.C. Ou, M.A. Mendoza, T. Lewandowski, and T. Kavanagh, nvestigations of methylmercury-induced alterations in neurogenesis. Environ Healt Perspect, 2002. 110 Suppl 5: p. 859-64

145. Welch-Carre, E., The neurodevelopmental consequences of prenatal alcohol exposure. Ad Neonatal Care, 2005. 5(4): p. 217-29.

46. Costa, L.G., V. Fattori, G. Giordano, and A. Vitalone, An in vitro approach to assess the toxicity of certain food contaminants: methylmercury and polychlorinated biphenyls. Toxicology, 2007. 237(1-3): p. 65-76.

147. Shafer, T.J., C.A. Meacham, and S. Barone, Jr., Effects of prolonged exposure to nanomolar concentrations of methylmercury on voltage-sensitive sodium and calcium currents in PC12 cells. Brain Res Dev Brain Res, 2002. 136(2): p. 151-64

148. Walum, E. and O.P. Flint, Midbrain micromass cultures: a model for studies of teratogenic and sub-teratogenic effects on CNS development. Acta Physiol Scand Suppl, 1990. 592: p. 61-72.

149. Mundy, W.R., N.M. Radio, and T.M. Freudenrich, Neuronal models for evaluation of proliferation in vitro using high content screening. Toxicology, 2010. 270(2-3): p. 121-30.

50. Radio, N.M. and W.R. Mundy, Developmental neurotoxicity testing in vitro: models for assessing chemical effects on neurite outgrowth. Neurotoxicology, 2008. 29(3): p. 361-76.

151. Robinson, J.F., W.C. Griffith, X. Yu, S. Hong, E. Kim, and E.M. Faustman, Methylmercury induced toxicogenomic response in C57 and SWV mouse embryos undergoing neural tube closure. Reprod Toxicol, 2010. 30(2): p. 284-91.

152. Robinson, J.F., Z. Guerrette, X. Yu, S. Hong, and E.M. Faustman, A systems-based approach to investigate dose- and time-dependent methylmercury-induced gene expression response in C57BL/6 mouse embryos undergoing neurulation. Birth Defects Res B Dev Reprod Toxicol, 2010. 89(3): p. 188-200.

153. Krewski, D., Toxicity Testing in the Twenty-first Century, C.o.T.a.A.o.E.A. NRC, Editor. 2007, National Academy of Sciences: Washington, DC.

154. Nemeth, K.A., A.V. Singh, and T.B. Knudsen, Searching for biomarkers of developmental toxicity with microarrays: normal eye morphogenesis in rodent embryos. Toxicol Appl Pharmacol, 2005. 206(2): p. 219-28.

155. van Dartel, D.A., J.L. Pennings, L.J. de la Fonteyne, K.J. Brauers, S. Claessen, J.H. van Delft, J.C. Kleinjans, and A.H. Piersma, Evaluation of developmental toxicant identification using gene expression profiling in embryonic stem cell differentiation cultures. Toxicol Sci, 2011. 119(1): p. 126-34.

156. Burbacher, T.M., P.M. Rodier, and B. Weiss, Methylmercury developmental neurotoxicity comparison of effects in humans and animals. Neurotoxicol Teratol, 1990. 12(3): 191-202.

157. Myers, G.J. and P.W. Davidson, Prenatal methylmercury exposure and children: neurologic, developmental, and behavioral research. Environ Health Perspect, 1998. 106 Suppl 3: p. 841-7.

158. Irizarry, R.A., B. Hobbs, F. Collin, Y.D. Beazer-Barclay, K.J. Antonellis, U. Scherf, and T.P. Speed, Exploration, normalization, and summaries of high density oligonucleotide array probe level data. Biostatistics, 2003. 4(2): p. 249-64.

59. Bolstad, B.M., R.A. Irizarry, M. Astrand, and T.P. Speed, A comparison of normalization methods for high density oligonucleotide array data based on variance and bias. Bioinformatics, 2003. 19(2): p. 185-93.
160. de Leeuw, W.C., H. Rauwerda, M.J. Jonker, and T.M. Breit, Salvaging Affymetrix probes after probe-level re-annotation. BMC Res Notes, 2008. 1: p. 66

161. Dai, M., et al., Evolving geneltranscript definitions significantly alter the interpretation of GeneChip data. Nucleic Acids Res, 2005. 33(20): p. e175.

162. Huang D.W., et al. The DAVID Gene Functional Classification Tool: a novel biological module-centric algorithm to functionally analyze large gene lists. Genome Biol, 2007. 8(9): p. R183.

163. Su, A.I., et al., A gene atlas of the mouse and human protein-encoding transcriptomes. Proc Natl Acad Sci U S A, 2004. 101(16): p. 6062-7.

164. Niwa, H., J. Miyazaki, and A.G. Smith, Quantitative expression of Oct-3/4 defines differ entiation, dedifferentiation or self-renewal of ES cells. Nat Genet, 2000. 24(4): p. 372-6.

165. Ogawa, K., et al., Activin-Nodal signaling is involved in propagation of mouse embryonic stem cells. J Cell Sci, 2007. 120(Pt 1): p. 55-65.

166. Liu, L., E.E. Geisert, A. Frankfurter, A.J. Spano, C.X. Jiang, J. Yue, I. Dragatsis, and D. Goldowitz, A transgenic mouse class-III beta tubulin reporter using yellow fluorescen protein. Genesis, 2007. 45(9): p. 560-9.

67. Moskowitz, P.F. and M.M. Oblinger, Transcriptional and post-transcriptional mechanism regulating neurofilament and tubulin gene expression during normal development of the rat brain. Brain Res Mol Brain Res, 1995. 30(2): p. 211-22.

68. Abu-Abed, S., P. Dolle, D. Metzger, B. Beckett, P. Chambon, and M. Petkovich, The retinoic acid-metabolizing enzyme, CYP26A1, is essential for normal hindbrain patterning, vertebral identity, and development of posterior structures. Genes Dev, 2001. 15(2): p. $226-40$.

169. Abranches, E., M. Silva, L. Pradier, H. Schulz, O. Hummel, D. Henrique, and E. Bekman, Neural differentiation of embryonic stem cells in vitro: a road map to neurogenesis in the embryo. PLoS One, 2009. 4(7): p. e6286.

170. Kim, M., A. Habiba, J.M. Doherty, J.C. Mills, R.W. Mercer, and J.E. Huettner, Regulation of mouse embryonic stem cell neural differentiation by retinoic acid. Dev Biol, 2009. 328(2): p. 456-71.

171. Mitiku, N. and J.C. Baker, Genomic analysis of gastrulation and organogenesis in the mouse. Dev Cell, 2007. 13(6): p. 897-907.

72. Ceccatelli, S., E. Dare, and M. Moors, Methylmercury-induced neurotoxicity and apoptosis. Chem Biol Interact, 2010. 188(2): p. 301-8.

173. Spyker, J.M. and M. Smithberg, Effects of methylmercury on prenatal development in mice. Teratology, 1972. 5(2): p. 181-90.

174. Pellizzer, C., S. Adler, R. Corvi, T. Hartung, and S. Bremer, Monitoring of teratogenic effects in vitro by analysing a selected gene expression pattern. Toxicol In Vitro, 2004. 18(3): p. 325-35.

175. Genschow, E., et al., The ECVAM international validation study on in vitro embryotoxicity tests: results of the definitive phase and evaluation of prediction models. European Centrc for the Validation of Alternative Methods. Altern Lab Anim, 2002. 30(2): p. 151-76.

176. Paquette, J.A., S.W. Kumpf, R.D. Streck, J.J. Thomson, R.E. Chapin, and D.B. Stedman, Assessment of the Embryonic Stem Cell Test and application and use in the pharmaceutical industry. Birth Defects Res B Dev Reprod Toxicol, 2008. 83(2): p. 104-11.

177. Theunissen, P.T., J.L. Pennings, J.F. Robinson, S.M. Claessen, J.C. Kleinjans, and A.H. Piersma, Time-response evaluation by transcriptomics of methylmercury effects on neural differentiation of murine embryonic stem cells. Toxicol Sci, 2011. 122(2): p. 437-47.

178. Becker, H., Teratogenicity study in rats with SAN619F. TCC AG itingen, Switzerland, Sandoz AG, Switzerland. (Unpublished), 1985.

179. Killick, M., G. Wickramaratne, P. Banham, and M. Thomas, PP523: Teratogenicity Study in the Rat. ICI Central Toxicology Laboratory, UK. ICI Agrochemicals, UK. (Unpublished), 1984 
180. Machera, K., Developmental toxicity of cyproconazole, an inbibitor of fungal ergosterol biosynthesis, in the rat. Bull Environ Contam Toxicol, 1995. 54(3): p. 363-9.

181. Duncan, S., Teratogenesis of sodium valproate. Curr Opin Neurol, 2007. 20(2): p. 175-80.

182. Meador, K., M.W. Reynolds, S. Crean, K. Fahrbach, and C. Probst, Pregnancy outcomes in women with epilepsy: a systematic review and meta-analysis of published pregnancy Epilepsy Res, 2008. 81(1): p. 1-13.

183. Theunissen, P.T., J.L. Pennings, J.F. Robinson, S.M. Claessen, J.C. Kleinjans, and A.H. Piersma, Time-response evaluation by transcriptomics of methylmercury effects on neural cstem cells. Toxicol Sci, 2011

184. Uibel, F., A. Muhleisen, C. Kohle, M. Weimer, T.C. Stummann, S. Bremer, and M. Schwarz, ReProGlo: a new stem cell-based reporter assay aimed to predict embryotoxic potential of drugs and chemicals. Reprod Toxicol, 2010. 30(1): p. 103-12.

185. Seiler, A., A. Visan, R. Buesen, E. Genschow, and H. Spielmann, Improvement of an in vitro stem cell assay for developmental toxicity: the use of molecular endpoints in the embryonic stem cell test. Reprod Toxicol, 2004. 18(2): p. 231-40.

186. Barrier, M., S. Jeffay, H.P. Nichols, K.J. Chandler, M.R. Hoopes, K. Slentz-Kesler, and E.S. Hunter, 3rd, Mouse embryonic stem cell adherent cell differentiation and cytotoxicity (ACDC) assay. Reprod Toxicol, 2011. 31(4): p. 383-91.

187. van Dartel, D.A., J.L. Pennings, F.J. van Schooten, and A.H. Piersma, Transcriptomics based identification of developmental toxicants through their interference with cardiomyocyte differentiation of embryonic stem cells. Toxicol Appl Pharmacol, 2010. $243(3):$ p. $420-8$.

188. Mehnert, J.M. and W.K. Kelly, Histone deacetylase inbibitors: biology and mechanism of action. Cancer J, 2007. 13(1): p. 23-9.

89. Bendall, A.J. and C. Abate-Shen, Roles for Msx and Dlx homeoproteins in vertebrate development. Gene, 2000. 247(1-2): p. 17-31.

190. Meno, C., Y. Saijoh, H. Fujii, M. Ikeda, T. Yokoyama, M. Yokoyama, Y. Toyoda, and H. Hamada, Left-right asymmetric expression of the TGF beta-family member lefty in mouse embryos. Nature, 1996. 381(6578): p. 151-5.

191. Stavridis, M.P., B.J. Collins, and K.G. Storey, Retinoic acid orchestrates fibroblast growth factor signalling to drive embryonic stem cell differentiation. Development, 2010. 137(6): p. 881-90.

192. Herrmann, B.G., S. Labeit, A. Poustka, T.R. King, and H. Lehrach, Cloning of the T gene required in mesoderm formation in the mouse. Nature, 1990. 343(6259): p. 617-22.

193. Duester, G., Retinoic acid synthesis and signaling during early organogenesis. Cell, 2008. 134(6): p. 921-31.

194. Yashiro, K., X. Zhao, M. Uehara, K. Yamashita, M. Nishijima, J. Nishino, Y. Saijoh, Y. Sakai, and $\mathrm{H}$. Hamada, Regulation of retinoic acid distribution is required for proximodistal patterning and outgrowth of the developing mouse limb. Dev Cell, 2004. 6(3): p. 411-22. Neurosci, 1997. 20: p. 483-532.

196. Ghannoum, M.A. and L.B. Rice, Antifungal agents: mode of action, mechanisms of resistance, and correlation of these mechanisms with bacterial resistance. Clin Microbio Rev, 1999. 12(4): p. 501-17.

197. Bolanos, J.P., J.M. Medina, and D.H. Williamson, Inbibition of sterol but not fatty acid synthesis by valproate in developing rat brain in vivo. Biochem J, 1990. 272(1): p. 251-3.

198. Gustavsen, M.W., K. von Krogh, E. Tauboll, K.E. Zimmer, E. Dahl, I. Olsaker, E. Ropstad, and S. Verhaegen, Differential effects of antiepileptic drugs on steroidogenesis in a human in vitro cell model. Acta Neurol Scand Suppl, 2009(189): p. 14-21.

199. Brion, L., A. Gorostizaga, N.V. Gomez, E.J. Podesta, F. Cornejo Maciel, and C. Paz Valproic 2011. 25(1): p. 7-12.
200. Glaser, K.B., M.J. Staver, J.F. Waring, J. Stender, R.G. Ulrich, and S.K. Davidsen, Gene expression profiling of multiple histone deacetylase (HDAC) inhibitors: defining a common gene set produced by HDAC inhibition in T24 and MDA carcinoma cell lines. Mol Cance Ther, 2003. 2(2): p. 151-63.

201. Gottlicher, M., et al., Valproic acid defines a novel class of HDAC inbibitors inducing differentiation of transformed cells. EMBO J, 2001. 20(24): p. 6969-78.

202. Phiel, C.J., F. Zhang, E.Y. Huang M.G. Guenther, M.A. Lazar, and P.S. Klein, Histone deacetylase is a direct target of valproic acid, a potent anticonvulsant, mood stabilizer, and teratogen. J Biol Chem, 2001. 276(39): p. 36734-41.

203. Theunissen, P.T., J.F. Robinson, J.L. Pennings, E. de Jong, S.M. Claessen, J.C. Kleinjans, and A.H. Piersma, Transcriptomic Concentration-Response Evaluation of Valproic Acid, Cyproconazole, and Hexaconazole in the Neural Embryonic Stem Cell Test (ESTn). Toxico Sci, $2012.125(2)$. p. $430-8$.

204. Azarbayjani, F., L.A. Borg, and B.R. Danielsson, Increased susceptibility to phenytoi teratogenicity: excessive generation of reactive oxygen species or impaired antioxidan defense? Basic Clin harmacol Toxicol, 2006.99(4): p. 305-11.

205. Jentink, J., H. Dolk, M.A. Loane, J.K. Morris, D. Wellesley, E. Garne, and L. de Jong-va den Berg, Intrauterine exposure to carbamazepine and specific congenital malformations: systematic review and case-control study. BMJ, 2010. 341: p. c6581.

206. Riley, E.P., M.A. Infante, and K.R. Warren, Fetal alcohol spectrum disorders: an overview. Neuropsychol Rev, 2011. 21(2): p. 73-80.

207. Shiota, K. and H. Nishimura, Teratogenicity of di(2-ethylhexyl) phthalate (DEHP) and di-n-butyl phthalate (DBP) in mice. Environ Health Perspect, 1982. 45: p. 65-70.

208. Yagi, Y., Y. Nakamura, I. Tomita, K. Tsuchikawa, and N. Shimoi, Teratogenic potential of di- and mono-(2-ethylhexyl)phthalate in mice. J Environ Pathol Toxicol, 1980. 4(2-3): p. $533-44$.

209. Kavlock, R., et al., NTP Center for the Evaluation of Risks to Human Reproduction phthalates expert panel report on the reproductive and developmental toxicity of di(2-ethylhexyl) phthalate. Reprod Toxicol, 2002. 16(5): p. 529-653.

210. Nicolai, J., J.S. Vles, and A.P. Aldenkamp, Neurodevelopmental delay in children exposed to antiepileptic drugs in utero: a critical review directed at structural study-bias. J Neuro Sci, 2008. 271(1-2): p. 1-14.

211. Farag, A.T. and H.H. Ibrahim, Developmental toxic effects of antifungal flusilazole administered by gavage to mice. Birth Defects Res B Dev Reprod Toxicol, 2007. 80(1): p. $12-7$.

212. Knudsen, T.B., M.T. Martin, R.J. Kavlock, R.S. Judson, D.J. Dix, and A.V. Singh, Profiling the activity of environmental chemicals in prenatal developmental toxicity studies using the U.S. EPA's ToxRefDB. Reprod Toxicol, 2009. 28(2): p. 209-19.

213. Czeizel, A.E., M. Rockenbauer, J. Olsen, and H.T. Sorensen, Oral phenoxymethylpenicillin treatment during pregnancy. Results of a population-based Hungarian case-control study. Arch Gynecol Obstet, 2000. 263(4): p. 178-81.

214. Oliveros, J.C. VENNY. An interactive tool for comparing lists with Venn Diagrams. http:// bioinfogp.cnb.csic.es/tools/venny/index.html. 2007.

215. Hermsen, S.A., T.E. Pronk, E.J. van den Brandhof, L.T. van der Ven, and A.H. Piersma, Chemical class-specific gene expression changes in the zebrafish embryo after exposure to Tecids and 1,2,4-triazole antifungals. Reprod Toxicol, 2011, 32(2): p. 245-52.

216. Yang, L., J.R. Kemadjou, C. Zinsmeister, M. Bauer, J. Legradi, F. Muller, M. Pankratz, J. Jakel, and U. Strahle, Transcriptional profiling reveals barcode-like toxicogenomi responses in the zebrafish embryo. Genome Biol, 2007. 8(10): p. R227.

217. NRC, Toxicity Testing in the 21st Century: A Vision and A Strategy., N.A. Press, Editor 2007: Washington, DC. 
218. Sylvie, J., C. Ellen, and V. Kris, The role of Wnt in cell signaling and cell adhesion during early vertebrate development. Front Biosci, 2011. 17: p. 2352-66.

219. Georgala, P.A., C.B. Carr, and D.J. Price, The role of Pax6 in forebrain development. Dev Neurobiol, 2011. 71(8): p. 690-709.

220. Lee, R.D., et al., Neurotoxic effects of alcohol and acetaldehyde during embryonic development. J Toxicol Environ Health A, 2005. 68(23-24): p. 2147-62.

221. Menegola, E., M.L. Broccia, F. Di Renzo, and E. Giavini, Antifungal triazoles induce malformations in vitro. Reprod Toxicol, 2001. 15(4): p. 421-7.

222. Menegola, E., M.L. Broccia, F. Di Renzo, V. Massa, and E. Giavini, Study on the common teratogenic pathway elicited by the fungicides triazole-derivatives. Toxicol In Vitro, 2005. 19(6): p. 737-48

223. Tiboni, G.M., F. Marotta, and E. Carletti, Fluconazole alters CYP26 gene expression in mouse embryos. Reprod Toxicol, 2009. 27(2): p. 199-202.

224. Pennimpede, T., D.A. Cameron, G.A. MacLean, H. Li, S. Abu-Abed, and M. Petkovich, The role of CYP26 enzymes in defining appropriate retinoic acid exposure during embryogenesis. Birth Defects Res A Clin Mol Teratol, 2010. 88(10): p. 883-94.

25. Marotta, F. and G.M. Tiboni, Molecular aspects of azoles-induced teratogenesis. Expert Opin Drug Metab Toxicol, 2010. 6(4): p. 461-82.

226. Boekelheide, K. and M.E. Andersen, A mechanistic redefinition of adverse effects - a key step in the toxicity testing paradigm shift. ALTEX, 2010. 27(4): p. 243-52.

27. Beutler, A.S., S. Li, R. Nicol, and M.J. Walsh, Carbamazepine is an inhibitor of histon deacetylases. Life Sci, 2005. 76(26): p. 3107-1

228. Van den Bossche, H., G. Willemsens, W. Cools, P. Marichal, and W. Lauwers, Hypothesis on the molecular basis of the antifungal activity of $\mathrm{N}$-substituted imidazoles and triazoles. Biochem Soc Trans, 1983. 11(6): p. 665-7.

229. Andersen, M.E., H.J. Clewell, 3rd, E. Bermudez, D.E. Dodd, G.A. Willson, J.L. Campbell, and R.S. Thomas, Formaldehyde: integrating dosimetry, cytotoxicity, and genomics to understand dose-dependent transitions for an endogenous compound. Toxicol Sci, 2010. 118(2): p. 716-31.

230. van Dartel, D.A., J.L. Pennings, L.J. de la Fonteyne, K.J. Brauers, S. Claessen, J.H. van Delft, J.C. Kleinjans, and A.H. Piersma, Concentration-dependent gene expression responses to flusilazole in embryonic stem cell differentiation cultures. Toxicol App Pharmacol, 2011. 251(2): p. 110-8.

231. Theunissen, P.T., J.F. Robinson, J.L. Pennings, M.H. van Herwijnen, J.C. Kleinjans, and A.H. Piersma, Compound-specific effects of diverse neurodevelopmental toxicants on global gene expression in the neural embryonic stem cell test (ESTn). Toxicol. Appl. Pharmacol., 2012. In press(0): p. 0.

232. Buesen, R., E. Genschow, B. Slawik, A. Visan, H. Spielmann, A. Luch, and A. Seiler, Embryonic stem cell test remastered: comparison between the validated EST and the new molecular FACS-EST for assessing developmental toxicity in vitro. Toxicol. Sci., 2009. 108(2): p. 389-400.

233. van Dartel, D.A., J.L. Pennings, L.J. de la Fonteyne, M.H. van Herwijnen, J.H. van Delft, F.J. van Schooten, and A.H. Piersma, Monitoring developmental toxicity in the embryonic tem cell test using differential Sci., 2010. 116(1): p. 130-139.

234. van Dartel, D.A., J.L. Pennings, F.J. van Schooten, and A.H. Piersma, Transcriptomics-based dentification of developmental toxicants through their interference with cardiomyocyte differentiation of embryonic stem cells. Toxicol. Appl. Pharmacol., 2010. 243(3): p. 420-428. 235. Aiba, K., et al., Defining developmental potency and cell lineage trajectories by expression
profiling of differentiating mouse embryonic stem cells. DNA Res, 2009. 16(1): p. 73-80.

6. R Development Core Team, R: A language and environment for statistical computing. 2011, R Foundation for Statistical Computing, Vienna, Austria.
237. Huang, D.W., B.T. Sherman, and R.A. Lempicki, Systematic and integrative analysis of large gene lists using DAVID bioinformatics resources. Nat. Protoc, 2009. 4(1): p. 44-57.

238. Liberzon, A., A. Subramanian, R. Pinchback, H. Thorvaldsdottir, P. Tamayo, and J.P. Mesirov, Molecular signatures database (MSigDB) 3.0. Bioinformatics, 2011. 27(12): p. $1739-40$.

239. Kuhn, M., D. Szklarczyk, A. Franceschini, M. Campillos, C. von Mering, L.J. Jensen, A Beyer, and P. Bork, STITCH 2: an interaction network database for small molecules and proteins. Nucleic Acids Res, 2010. 38(Database issue): p. D552-6.

240. Shannon, P. A. Markiel, O. Ozier, N.S. Baliga, J.T. Wang, D. Ramage, N. Amin, B. Schwikowski, and T. Ideker, Cytoscape: a software environment for integrated models of biomolecular interaction networks. Genome Res., 2003. 13(11): p. 2498-2504.

241. Ivanova, N., R. Dobrin, R. Lu, I. Kotenko, J. Levorse, C. DeCoste, X. Schafer, Y. Lun, and I.R. Lemischka, Dissecting self-renewal in stem cells with RNA interference. Nature, 2006 $442(7102)$ : p. $533-8$.

242. Zhou, Q., H. Chipperfield, D.A. Melton, and W.H. Wong, A gene regulatory network i mouse embryonic stem cells. Proc Natl Acad Sci U S A, 2007. 104(42): p. 16438-43.

43. Boyer, L.A., et al., Polycomb complexes repress developmental regulators in murin embryonic stem cells. Nature, 2006. 441(7091): p. 349-53

244. Lee, G., H. Kim, Y. Elkabetz, G. Al Shamy, G. Panagiotakos, T. Barberi, V. Tabar, and L. Studer, Isolation and directed differentiation of neural crest stem cells derived from huma embryonic stem cells. Nat Biotechnol, 2007. 25(12): p. 1468-75.

45. Segal, E., N. Friedman, D. Koller, and A. Regev, A module map showing conditional activit of expression modules in cancer. Nat.Genet., 2004. 36(10): p. 1090-1098.

ane, secreted and GPI-anchored semaphorins in vertebrates. Cell, 1999. 99(1): p. 71-80.

247. Rohm, B., A. Ottemeyer, M. Lohrum, and A.W. Puschel, Plexin/neuropilin complexes mediate repulsion by the axonal guidance signal semaphorin 3A. Mech Dev, 2000. 93(1-2) p. 95-104.

248. McIntyre, J.C., W.B. Titlow, and T.S. McClintock, Axon growth and guidance genes identify nascent, immature, and mature olfactory sensory neurons. J Neurosci Res, 2010. 88(15): p. 3243-56

249. Broer, A., N. Tietze, S. Kowalczuk, S. Chubb, M. Munzinger, L.K. Bak, and S. Broer, The orphan transporter v7-3 (slc6a15) is a Na+-dependent neutral amino acid transporter (B0AT2). Biochem J, 2006. 393(Pt 1): p. 421-30.

250. Farmer, M.K., et al., Cloning and characterization of human NTT5 and v7-3: two orphan transporters of the $\mathrm{Na}+\mathrm{Cl}$ - -dependent neurotransmitter transporter gene family. Genomics, 2000. 70(2): p. 241-52

251. Kohli, M.A., et al., The neuronal transporter gene SLC6A15 confers risk to major depression. Neuron, 2011. 70(2): p. 252-65.

252. Jokela, H., et al., Hydroxysteroid (17beta) dehydrogenase 7 activity is essential for fetal de novo cholesterol synthesis and for neuroectodermal survival and cardiovascular differentiation in early mouse embryos. Endocrinology, 2010. 151(4): p. 1884-92.

253. Wolf, G., The function of cholesterol in embryogenesis. J Nutr Biochem, 1999. 10(4): p. $188-92$

254. Beachy, P.A., M.K. Cooper, K.E. Young, D.P. von Kessler, W.J. Park, T.M. Hall, D.J. Leahy, and J.A. Porter, Multiple roles of cholesterol in hedgehog protein biogenesis and signaling. Cold Spring Harb Symp Quant Biol, 1997. 62: p. 191-204.

255. Salen, G., S. Shefer, A.K. Batta, G.S. Tint, G. Xu, A. Honda, M. Irons, and E. R. Elias, . 37(6): p. 1169-80.

256. Tozawa, R., et al., Embryonic lethality and defective neural tube closure in mice lackin squalene synthase. J Biol Chem, 1999. 274(43): p. 30843-8. 
257. Killick, M., Wickramaratne, G., Banham, P. and Thomas, M., PP523: Teratogenicity Study in the Rat, U. ICI Central Toxicology Laboratory, Editor. 1984, ICI Central Toxicology Laboratory, UK. ICI Agrochemicals, UK.

258. Steiner, G., L. Suter, F. Boess, R. Gasser, M.C. de Vera, S. Albertini, and S. Ruepp, Discriminating different classes of toxicants by transcript profiling. Environ Health Perspect, 2004. 112(12): p. 1236-48.

259. Lamb, J., et al., The Connectivity Map: using gene-expression signatures to connect small molecules, genes, and disease. Science, 2006. 313(5795): p. 1929-35.

260. U.S.EPA, An Approach to Using Toxicogenomic Data in U.S. EPA Human Health Risk Assessments: A Dibutyl Phthalate Case Study. 2009, National Center for Environmental Assessment: Washington, DC

261. van der Jagt, K., J. Tørsløv, and J. de Bruijn, Alternative approaches can reduce the use of test animals under REACH. 2004, European Commission Report.

262. Engberg, N., M. Kahn, D.R. Petersen, M. Hansson, and P. Serup, Retinoic acid synthesis promotes development of neural progenitors from mouse embryonic stem cells by suppressing endogenous, Wht-dependent nodal signaling. Stem Cells, 2010. 28(9): p 1498-509.

263. Theunissen, P.T., J.L. Pennings, J.F. Robinson, M. Herwijnen, J.C. Kleinjans, and A. Piersma, Compound-specific effects of diverse neurodevelopmental toxicants on global gene expression in the neural embryonic stem cell test (ESTn) Toxicol Appl Pharmacol, 2012.

264. Schenk, B., et al., The ReProTect Feasibility Study, a novel comprehensive in vitro approach to detect reproductive toxicants. Reprod Toxicol, 2010. 30(1): p. 200-18.

265. Kavlock, R., et al., Update on EPA's ToxCast Program: Providing High Throughpu Decision Support Tools for Chemical Risk Management. Chem Res Toxicol, 2012.

266. Sipes, N.S., et al., Predictive models of prenatal developmental toxicity from Tox Cast high throughput screening data. Toxicol Sci, 2011. 124(1): p. 109-27.

267. Chandler, K.J., et al., Evaluation of 309 environmental chemicals using a mouse embryonic stem cell adherent cell differentiation and cytotoxicity assay. PLoS One, 2011. 6(6): p. e18540.

268. Padilla, S., et al., Zebrafish developmental screening of the ToxCast Phase I chemical library. Reprod Toxicol, 2012. 33(2): p. 174-87.

269. van der Burg, B., E.D. Kroese, and A.H. Piersma, Towards a pragmatic alternative testing strategy for the detection of reproductive toxicants. Reprod Toxicol, 2011. 31(4): p. 558-61.

270. Sedjo, R.L., J. Ranger-Moore, J. Foote, N.E. Craft, D.S. Alberts, M.J. Xu, and A.R. Giuliano, Circulating endogenous retinoic acid concentrations among participants enrolled in a randomized placebo-controlled clinical trial of retinyl palmitate. Cancer Epidemio Biomarkers Prev, 2004. 13(11 Pt 1): p. 1687-92.

271. Schneider, R.A., D. Hu, and J.A. Helms, From head to toe: conservation of molecular signals regulating limb and craniofacial morphogenesis. Cell Tissue Res, 1999. 296(1): 103-9.

272. Maden, M., E. Gale, and M. Zile, The role of vitamin A in the development of the central nervous system. J Nutr, 1998. 128(2 Suppl): p. 471S-475S.

273. Zile, M.H.,Vitamin A and embryonic development: an overview. J Nutr, 1998. 128 Suppl): p. $455 \mathrm{~S}-458 \mathrm{~S}$.

74. Rhinn, M. and P. Dolle, Retinoic acid signalling during development. Development, 2012. 139(5): p. 843-58.

275. Guan, K., H. Chang, A. Rolletschek, and A.M. Wobus, Embryonic stem cell-derived neurogenesis. Retinoic acid induction and lineage selection of neuronal cells. Cell Tissue Res, 2001. 305(2): p. 171-6.

276. Kudoh, T., S.W. Wilson, and I.B. Dawid, Distinct roles for Fgf, Wht and retinoic acid in posteriorizing the neural ectoderm. Development, 2002. 129(18): p. 4335-46.
277. Peters, A.K., M. Steemans, E. Hansen, N. Mesens, G.R. Verheyen, and P. Vanparys, Evaluation of the embryotoxic potency of compounds in a newly revised high throughput embryonic stem cell test. Toxicol Sci, 2008. 105(2): p. 342-50.

278. Bhattacharya, S., Q. Zhang, P.L. Carmichael, K. Boekelheide, and M.E. Andersen, Toxicit testing in the 21 century: defining new risk assessment approaches based on perturbation of intracellular toxicity pathways. PLoS One, 2011. 6(6): p. e20887.

279. Robinson, J.F., X. Yu, E.G. Moreira, S. Hong, and E.M. Faustman, Arsenic- and cadmium induced toxicogenomic response in mouse embryos undergoing neurulation. Toxicol App Pharmacol, 2011. 250(2): p. 117-29.

280. Luijten, M., V.A. van Beelen, A. Verhoef, M.F. Renkens, M.H. van Herwijnen, A. Westerman, F.J. van Schooten, J.L. Pennings, and A.H. Piersma, Transcriptomics analysis of retinoic acid embryotoxicity in rat postimplantation whole embryo culture. Reprod Toxicol, 30(2): $333-40$

281. Jennen, D.G., C. Magkoufopoulou, H.B. Ketelslegers, M.H. van Herwijnen, J.C. Kleinjans, and J.H. van Delft, Comparison of HepG2 and HepaRG by whole-genome gene expression analysis for the purpose of chemical hazard identification. Toxicol Sci, 2010. 115(1): p. 66-79.

282. Robinson, J.F., X. Yu, S. Hong, W.C. Griffith, R. Beyer, E. Kim, and E.M. Faustman, Cadmium-induced differential toxicogenomic response in resistant and sensitive mouse strain undergoing neurulation. Toxicol Sci, 2009. 107(1): p. 206-19.

283. Wlodarczyk, B.J., R.M. Cabrera, D.S. Hill, D. Bozinov, H. Zhu, and R.H. Finnell, Arsenic induced gene expression changes in the neural tube of folate transport defective mouse embryos. Neurotoxicology, 2006. 27(4): p. 547-57.

284. Boverhof, D.R., L.D. Burgoon, C. Tashiro, B. Sharratt, B. Chittim, J.R. Harkema, D.I Mendrick, and T.R. Zacharewski, Comparative toxicogenomic analysis of the hepatotoxic effects of TCDD in Sprague Dawley rats and C57BL/6 mice. Toxicol Sci, 2006. 94(2): p. 398-416.

285. Kienhuis, A.S., M.C. van de Poll, C.H. Dejong, R. Gottschalk, M. van Herwijnen, A. Boorsma, J.C. Kleinjans, R.H. Stierum, and J.H. van Delft, A toxicogenomics-based parallelogram approach to evaluate the relevance of coumarin-induced responses in primary human hepatocytes in vitro for humans in vivo. Toxicol In Vitro, 2009. 23(6): p. $1163-9$.

286. Silkworth, J.B., E.A. Carlson, C. McCulloch, K. Illouz, S. Goodwin, and T.R. Sutter, Toxicogenomic analysis of gender, chemical, and dose effects in livers of TCDD- or aroclor 1254-exposed rats using a multifactor linear model. Toxicol Sci, 2008. 102(2): p. 291-309.

287. Hendriksen, P.J., A.P. Freidig, D. Jonker, U. Thissen, J.J. Bogaards, M.M. Mumtaz, J.P. Groten, and R.H. Stierum, Transcriptomics analysis of interactive effects of benzene, trichloroethylene and methyl mercury within binary and ternary mixtures on the liver and kidney following subchronic exposure in the rat. Toxicol Appl Pharmacol, 2007. 225(2): p. 171-88.

288. Bosotti, R., G. Locatelli, S. Healy, E. Scacheri, L. Sartori, C. Mercurio, R. Calogero, and A. Isacchi, Cross platform microarray analysis for robust identification of differentially expressed genes. BMC Bioinformatics, 2007. 8 Suppl 1: p. S5.

289. Shi, L., R.G. Perkins, H. Fang, and W. Tong, Reproducible and reliable microarray results through quality control: good laboratory proficiency and appropriate data analysis practices are essential. Curr Opin Biotechnol, 2008. 19(1): p. 10-8.

290. Waters, M.D., M. Jackson, and I. Lea, Characterizing and predicting carcinogenicity and mode of action using conventional and toxicogenomics methods. Mutat Res, 2010.705(3): mode of action
p. $184-200$.

291. Glover, C.N., D. Zheng, S. Jayashankar, G.D. Sales, C. Hogstrand, and A.K. Lundebye, Methylmercury speciation influences brain gene expression and behavior in gestationallyexposed mice pups. Toxicol Sci, 2009. 110(2): p. 389-400. 
292. Yu, X., J.F. Robinson, J.S. Sidhu, S. Hong, and E.M. Faustman, A system-based comparison of gene expression reveals alterations in oxidative stress, disruption of ubiquitin-proteasome system and altered cell cycle regulation after exposure to cadmium and methylmercury in mouse embryonic fibroblast. Toxicol Sci, 2010. 114(2): p. 356-77.

293. Simon, R., A. Lam, M.C. Li, M. Ngan, S. Menenzes, and Y. Zhao, Analysis of gene expression data using BR B-ArrayTools. Cancer Inform, 2007. 3: p. 11-7.

294. Dennis, G., Jr., B.T. Sherman, D.A. Hosack, J. Yang, W. Gao, H.C. Lane, and R.A. Lempicki, DAVID: Database for Annotation, Visualization, and Integrated Discovery. Genome Biol, 2003. 4(5): p. P3.

295. Carbon, S., A. Ireland, C.J. Mungall, S. Shu, B. Marshall, and S. Lewis, AmiGO: online access to ontology and annotation data. Bioinformatics, 2009. 25(2): p. 288-9.

296. Saeed, A.I., et al., TM4: a free, open-source system for microarray data management and analysis. Biotechniques, 2003. 34(2): p. 374-8.

297. Harris, M.J. and D.M. Juriloff, Mouse mutants with neural tube closure defects and their role in understanding human neural tube defects. Birth Defects Res A Clin Mol Terato 2007. 79(3): p. 187-210.

298. Harris, M.J. and D.M. Juriloff, An update to the list of mouse mutants with neural tub closure defects and advances toward a complete genetic perspective of neural tube closure. Birth Defects Res A Clin Mol Teratol, 2010. 88(8): p. 653-69.

299. Heuer, J., S. Bremer, I. Pohl, and H. Spielmann, Development of an in vitro embryotoxicit test using murine embryonic stem cell cultures. Toxicol In Vitro, 1993. 7(4): p. 551-6.

300. van Delft, J.H., E. van Agen, S.G. van Breda, M.H. Herwijnen, Y.C. Staal, and J.C. Kleinjans, Discrimination of genotoxic from non-genotoxic carcinogens by gene expression profiling. Carcinogenesis, 2004. 25(7): p. 1265-76.

301. Hurd, E.A., P.L. Capers, M.N. Blauwkamp, M.E. Adams, Y. Raphael, H.K. Poucher, an D.M. Martin, Loss of Chd7 function in gene-trapped reporter mice is embryonic lethal and associated with severe defects in multiple developing tissues. Mamm Genome, 2007. 18(2): p. 94-104.

302. Qu, Y., et al., Atypical cadherins Celsr1-3 differentially regulate migration of facial branchiomotor neurons in mice. J Neurosci, 2010. 30(28): p. 9392-401.

03. Su, M.Q. and G.T. Okita, Embryocidal and teratogenic effects of methylmercury in mice. Toxicol Appl Pharmacol, 1976. 38(1): p. 207-16.

304. Currie, R.A., et al., Gene ontology mapping as an unbiased method for identifying molecular pathways and processes affected by toxicant exposure: application to acute effects caused by the rodent non-genotoxic carcinogen diethylhexylphthalate. Toxicol Sci, 2005. 86(2): p. 453-69.

305. Kienhuis, A.S., et al., Parallelogram approach using rat-human in vitro and rat in vivo toxicogenomics predicts acetaminophen-induced hepatotoxicity in humans. Toxicol Sci, 2009. 107(2): p. $544-52$

306. Wobus, A.M. and P. Loser, Present state and future perspectives of using pluripotent stem cells in toxicology research. Arch Toxicol, 2011. 85(2): p. 79-117.

307. Mogi, A., H. Ichikawa, C. Matsumoto, T. Hieda, D. Tomotsune, S. Sakaki, S. Yamada, and K. Sasaki, The method of mouse embryoid body establishment affects structure and developmental gene expression. Tissue Cell, 2009. 41(1): p. 79-84.

308. Osakada, F. and M. Takahashi, Neural induction and patterning in Mammalian pluripotent stem cells. CNS Neurol Disord Drug Targets, 2011. 10(4): p. 419-32.

309. Darmon, M., W.B. Stallcup, and Q.J. Pittman, Induction of neural differentiation by serum deprivation in cultures of the embryonal carcinoma cell line 1003. Exp Cell Res, 1982. 138(1): p. 73-8.

310. Yamada, T., N. Suzuki, N. Hiraoka, K. Matsuoka, S. Fukushima, A. Hashiguchi, and J. Hata, Apoptosis of human embryonal carcinoma cells with in vitro differentiation. Cell Struct Funct, 1996. 21(1): p. 53-61.
311. Czyz, J. and A. Wobus, Embryonic stem cell differentiation: the role of extracellular factors. Differentiation, 2001. 68(4-5): p. 167-74.

312. De Miguel, M.P., S. Fuentes-Julian, and Y. Alcaina, Pluripotent stem cells: origin, maintenance and induction. Stem Cell Rev, 2010. 6(4): p. 633-49.

313. Ahn, J.I., K.H. Lee, D.M. Shin, J.W. Shim, J.S. Lee, S.Y. Chang, Y.S. Lee, M.J. Brownstein, and S.H. Lee, Comprehensive transcriptome analysis of differentiation of embryonic stem cells into midbrain and hindbrain neurons. Dev Biol, 2004. 265(2): p. 491-501.

314. Doss, M.X., et al., Global transcriptome analysis of murine embryonic stem cell-derived cardiomyocytes. Genome Biol, 2007. 8(4): p. R56.

315. Rolletschek, A. I.S. Schroeder, H. Schulz, O Hummel, N. Huebner, and A.M. Wobus, Characterization of mouse embryonic stem cell differentiation into the pancreatic lineage in vitro by transcriptional profiling, quantitative RT-PCR and immunocytochemistry. Int

316. Fang, H., Y. Yang, C. Li, S. Fu, Z. Yang, G. Jin, K. Wang, J. Zhang, and Y. Jin, Transcriptome analysis of early organogenesis in human embryos. Dev Cell, 2010. 19(1): p. 174-84.

17. O'Farrell, P.H., J. Stumpff, and T.T. Su, Embryonic cleavage cycles: how is a mouse like fly? Curr Biol, 2004. 14(1): p. R35-45.

18. Andersen, M.E. and D. Krewski, Toxicity testing in the 21st century: bringing the vision to life. Toxicol Sci, 2009. 107(2): p. 324-30.

19. van Thriel, C., R.H. Westerink, C. Beste, A.S. Bale, P.J. Lein, and M. Leist, Translating neurobehavioural endpoints of developmental neurotoxicity tests into in vitro assays and readouts. Neurotoxicology, 2011

20. Dingemans, M.M., A. de Groot, R.G. van Kleef, A. Bergman, M. van den Berg, H.P. $V_{1 j v e r b e r g}$, and R.H. Westerink, Hydroxylation increases the neurotoxic potential of BDE-47 to affect exocytosis and calcium homeostasis in PC12 cells. Environ Healt Perspect, 2008. 116(5): p. 637-43

321. Hogberg, H.T., A. Kinsner-Ovaskainen, S. Coecke, T. Hartung, and A.K. Bal-Price, $m$ RNA expression is a relevant tool to identify developmental neurotoxicants using an in vitro approach. Toxicol Sci, 2010. 113(1): p. 95-115.

22. Seiler, A.E. and H. Spielmann, The validated embryonic stem cell test to predic embryotoxicity in vitro. Nat Protoc, 2011. 6(7): p. 961-78.

323. Anoopkumar-Dukie, S., J.B. Carey, T. Conere, E. O'Sullivan, F.N. van Pelt, and A. Allshire, Resazurin assay of radiation response in cultured cells. Br J Radiol, 2005. 78(934): p. 945-7.

324. Kramer, N.I., J.C. van Eijkeren, and J.L. Hermens, Influence of albumin on sorption kinetics in solid-phase microextraction: consequences for chemical analyses and uptake processes. Anal Chem, 2007. 79(18): p. 6941-8.

325. Naciff, J.M., M.L. Jump, S.M. Torontali, G.J. Carr, J.P. Tiesman, G.J. Overmann, and G.P. Daston, Gene expression profile induced by 17alpha-ethynyl estradiol, bisphenol A, and genistein in the developing female reproductive system of the rat. Toxicol Sci, 2002. 68(1): p. 184-99.

326. Naciff, J.M., Z.S. Khambatta, T.D. Reichling, G.J. Carr, J.P. Tiesman, D.W. Singleton, S.A. Khan, and G.P. Daston, The genomic response of Ishikawa cells to bisphenol A exposure is dose- and time-dependent. Toxicology, 2010. 270(2-3): p. 137-49.

327. Naciff, J.M., G.J. Overmann, S.M. Torontali, G.J. Carr, J.P. Tiesman, B.D. Richardson, and G.P. Daston, Gene expression profile induced by 17 alpha-ethynyl estradiol in the prepubertal female reproductive system of the rat. Toxicol Sci, 2003. 72(2): p. 314-30.

328. Kienhuis, A.S., J.G. Bessems, J.L. Pennings, M. Driessen, M. Luiiten, J.H. van Delft, A.A. Peijnenburg, and L.T. van der Ven, Application of toxicogenomics in hepatic systems toxicology for risk assessment: acetaminophen as a case study. Toxicol Appl Pharmacol, 2011. 250(2): p. 96-107.

329. Piersma, A.H., L.G. Hernandez, J. van Benthem, J.J. Muller, F.X. van Leeuwen, T.G. Vermeire, and M.T. van Raaij, Reproductive toxicants have a threshold of adversity. Crit Rev Toxicol, 2011. 41(6): p. 545-54. 
330. Leeuwen, C. and J. Hermes, Risk assessment of chemicals: an introduction. 2nd ed. 2007: Kluwer Acadmeci Publishers. 688.

331. van Leeuwen, C., G. Patlewicz, and A. Worth, Intelligent Testing Strategies In: Risk Assessment of Chemicals. An Introduction. 2nd Edition ed, ed. C. Van Leeuwen and T. Vermeire. 2007, Dordrecht, The Netherlands: Springer Publishers.

332. Dix, D.J., K.A. Houck, M.T. Martin, A.M. Richard, R.W. Setzer, and R.J. Kavlock, The ToxCast program for prioritizing toxicity testing of environmental chemicals. Toxicol Sci,

2007. 95(1): p. 5-12.
333. Schardein, J., Chemically induced birth defects. Third ed. 2000, New York: Marcel Dekker

334. de Jong, E., A.M. Doedee, M.A. Reis-Fernandes, H. Nau, and A.H. Piersma, Potency ranking of valproic acid analogues as to inbibition of cardiac differentiation of embryonic stem cells in comparison to their in vivo embryotoxicity. Reprod Toxicol, 2011. 31(4): p.

335. de Jong, E., J. Louisse, M. Verwei, B.J. Blaauboer, J.J. van de Sandt, R.A. Woutersen, I.M. Rietjens, and A.H. Piersma, Relative developmental toxicity of glycol ether alkoxy acid metabolites in the embryonic stem cell test as compared with the in vivo potency of their parent compounds. Toxicol Sci, 2009. 110(1): p. 117-24.

336. Janer, G., A. Verhoef, H.D. Gilsing, and A.H. Piersma, Use of the rat postimplantation embryo culture to assess the embryotoxic potency within a chemical category and to identify toxic metabolites. Toxicol In Vitro, 2008. 22(7): p. 1797-805.

337. Schulpen, S.H., J.F. Robinson, J.L. Pennings, D.A. van Dartel, and A.H. Piersma, Dose response analysis of monophthalates in the murine embryonic stem cell test assessed by cardiomyocyte differentiation and gene expression. Reprod Toxicol, 2012.

338. Andersen, M.E., J.E. Dennison, R.S. Thomas, and R.B. Conolly, New directions in incidence-dose modeling. Trends Biotechnol, 2005. 23(3): p. 122-7.

39. Irie, N. and S. Kuratani, Comparative transcriptome analysis reveals vertebrate phylotypic period during organogenesis. Nat Commun, 2011. 2: p. 248.

340. Kultima, K., M. Jergil, H. Salter, A.L. Gustafson, L. Dencker, and M. Stigson, Early transcriptional responses in mouse embryos as a basis for selection of molecular markers predictive of valproic acid teratogenicity. Reprod Toxicol, 2010. 30(3): p. 457-68.

341. Robinson, J.F., A. Verhoef, and A. Piersma, Transcriptomic analysis of neurulation and early organogenesis in rat embryos: an in vivo and ex vivo comparison. . Toxicol Sci, 2011.

342. Robinson, J.F., P.T. Theunissen, D.A. van Dartel, J.L. Pennings, E.M. Faustman, and A.H. Piersma, Comparison of $\mathrm{MeHg}$-induced toxicogenomic responses across in vivo and in vitro models used in developmental toxicology. Reprod Toxicol, 2011. 32(2): p. 180-8.

\section{inderser}




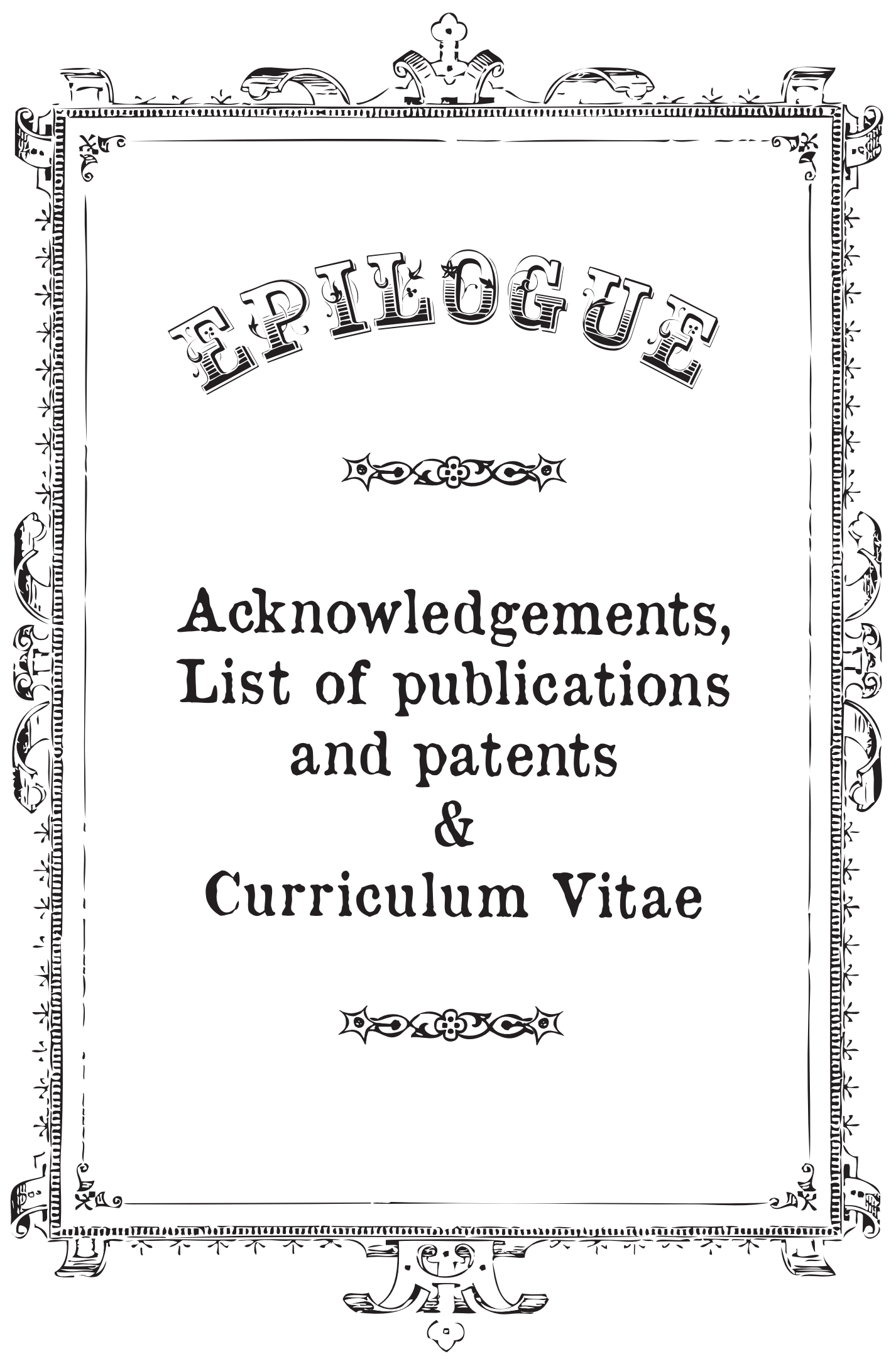




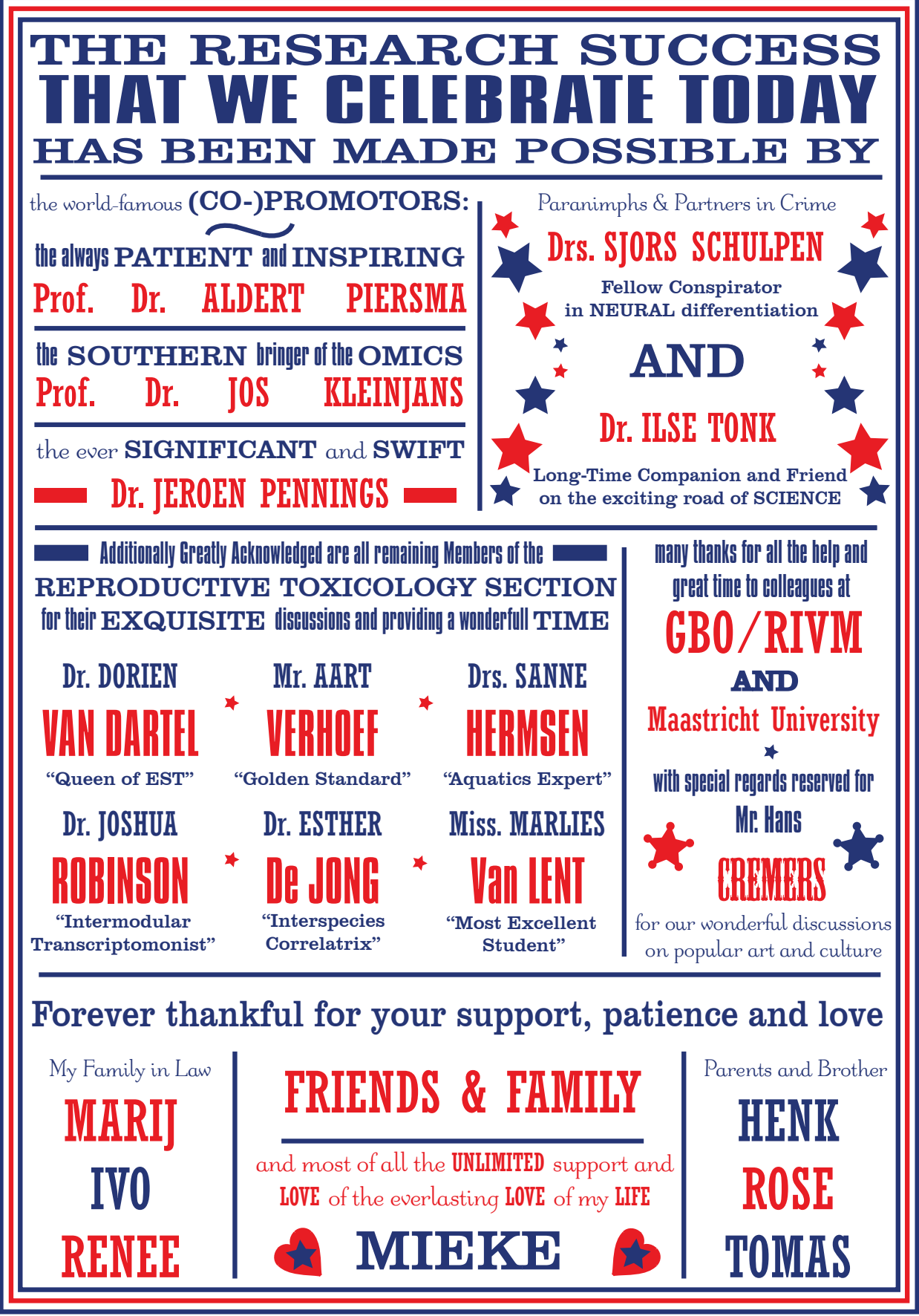




\section{List of publications and patents}

Theunissen PT, Pennings JL, van Dartel DAM, Robinson JF, Kleinjans JC, Piersma AH. Complementary detection of embryotoxic properties of substances in the neural and cardiac embryonic stem cell tests (ESTn and ESTc).

Accepted for Publication in Toxicological Sciences

Theunissen PT, de Jong E, Robinson JF, Piersma AH. Embryonic stem cell test (EST): molecular endpoints towards high-throughput analysis of chemical embryotoxic potential. In: Steinberg P, editor. High-throughput screening methods in toxicity testing. April 2013. Wiley \& Sons, inc.

Theunissen PT, Pennings JL, Piersma AH, inventors; University Maastricht, National Institute for Public Health and the Environment (RIVM) assignee. Method for determining the neurodevelopmental toxicity of a compound in vitro. European patent number EP 2518159; International patent number WO2012/146496. October 31 and November 1, 2012.

Pennings JL, Theunissen PT and Piersma AH. An optimized gene set for transcriptomics-based neurodevelopmental toxicity prediction in the neural embryonic stem cell test (ESTn)

Toxicology. (2012) 300(3):158-67.

Theunissen PT, Robinson JF, Pennings JL, van Herwijnen MH, Kleinjans JC, Piersma AH. Compound-specific effects of diverse neurodevelopmental toxicants on global gene expression in the neural embryonic stem cell test (ESTn).

Toxicology and Applied Pharmacology. (2012) 262(3):330-40.

Theunissen PT, Pennings JL, Piersma AH, inventors; University Maastricht, National Institute for Public Health and the Environment (RIVM) assignee. Method for determining the neurodevelopmental toxicity of a compound in vitro. European patent EP 12164410.8. April 17, 2012.

Theunissen PT \& Piersma AH. Innovative Approaches in the embryonic stem cell test (EST). Frontiers in Bioscience (2012) 17:1965-75. 
Theunissen PT, Robinson JF, Pennings JL, de Jong E, Claessen SM, Kleinjans JC, Piersma AH. Transcriptomic concentration-response evaluation of Valproic Acid, Cyproconazole and Hexaconazole in the neural embryonic stem cell test (ESTn).

Toxicological Sciences (2011) 125: 430-8.

Robinson JF, Theunissen PT, van Dartel DA, Pennings JL, Faustman EM, Piersma AH. Comparison of methylmercury-induced toxicogenomic responses in in vivo and in vitro models used in developmental toxicology.

Reproductive Toxicology (2011) 32: 180-8.

Theunissen PT, Pennings JL, Robinson JF, Claessen SM, Kleinjans JC, Piersma AH. Time-response evaluation by transcriptomics of methylmercury effects on neural differentiation of murine embryonic stem cells.

Toxicological Sciences (2011) 122:437-47.

Theunissen PT, Schulpen SH, van Dartel DA, Hermsen SA, van Schooten FJ, Piersma AH. An abbreviated protocol for multi-lineage neural differentiation of murine embryonic stem cells and its perturbation by methylmercury Reproductive Toxicology (2010) 29(4):383-92.

Health Council of the Netherlands. Report preparation by Theunissen PT, Wolterbeek APM. Hydrogen fluoride and sodium fluoride: Evaluation of the effects on reproduction, recommendation for classification. The Hague: Health Council of the Netherlands, 2009; publication no. 2009/OSH04.

Mahabir AG, Schaap M, Theunissen PT, van Benthem J, Essers J, de Vries A, Hendriksen CF, van Steeg H. DNA-repair-deficient Rad54/Rad54B mice are more sensitive to clastogens than wild-type mice.

Toxicology Letters (2008) 183 (1-3): 112-7.

\section{Lipexist}

\section{Curriculum Vitae}

Peter T. Theunissen was born on April 14 $4^{\text {th }}, 1983$ in Nijmegen, the Netherlands. He grew up in Mook where he attended elementary school. After he graduated rom secondary education at Stedelijk Gymnasium Nijmegen in 2001, he studied Biomedical Sciences at the Radboud University Nijmegen from 2001 until 2006 with a major in Toxicology and minors in Neuropharmacology and Reproduction. He conducted his Master internship Toxicology at the laboratory of Toxicology, Pathology and Genetics at the National Institute for Public Health and the Environment (RIVM) under supervision of Anuska Mahabir and Jan van Benthem. Furthermore, he performed a minor internship Neuropharmacology at the departments Psychoneuropharmacology and Cel Physiology at the Radboud University Nijmegen Medical Centre (RUNMC) nd Nijmegen Centre for Molecular Life Sciences (NCMLS) under supervision of Jocelien Olivier, Peter Deen and Bart Ellenbroek. His second mino internship Reproduction was conducted at the department of Toxicology and Applied Pharmacology at TNO Quality of Life, under supervision of Mariska Tegelenbosch. After obtaining his Master s degree in december 2006, Peter worked as a research assistant in the field of reproductive toxicology at TNO Quality of Life, under supervision of André Wolterbeek. In September 2007, he started as a PhD student at the department of Health Risk Analysis and Toxicology and the department of Toxicogenomics at Maastricht University. The project was in cooperation with the laboratory for Health Protection Research at the RIVM, where he was stationed. During this research he completed the Postdoctoral Education in Toxicology, which will result in a European registration as Toxicologist.

Since February 2012 Peter works as a postdoctoral fellow at the department Innovative Testing at the Utrecht University of Applied Science (Hogeschool Utrecht), the laboratory for Health Protection Research at the RIVM and the Dutch medicines evaluation board (CBG-MEB).

\section{Lipesesers}


